\title{
HIN-1, an inhibitor of cell growth, invasion, and AKT1 activation
}

\section{Citation}

Krop, IE, MT Parker, N Qimron, D Porter, and K Polyak. 2005. “HIN-1, an inhibitor of cell growth, invasion, and AKT1 activation." Breast Cancer Research : BCR 7 (Suppl 2): P4.04. doi:10.1186/ bcr1134. http://dx.doi.org/10.1186/bcr1134.

\section{Published Version}

doi: $10.1186 /$ bcr1134

\section{Permanent link}

http://nrs.harvard.edu/urn-3:HUL.InstRepos:13454771

\section{Terms of Use}

This article was downloaded from Harvard University's DASH repository, and is made available under the terms and conditions applicable to Other Posted Material, as set forth at http:// nrs.harvard.edu/urn-3:HUL.InstRepos:dash.current.terms-of-use\#LAA

\section{Share Your Story}

The Harvard community has made this article openly available.

Please share how this access benefits you. Submit a story.

\section{Accessibility}



Molecular Biology of Breast Cancer

\author{
Molde, Norway \\ 22-26 June 2005
}

Received: 15 April 2005 Published: 17 June 2005

(c) 2005 BioMed Central Ltd

\section{Speaker abstracts}

\section{S.01}

The challenges in translating present knowledge of the molecular biology of breast cancer into clinical use

\section{N Davidson}

Johns Hopkins University, USA

Breast Cancer Research 2005, 7(Suppl 2):S.01 (DOI 10.1186/bcr1044)

Abstract not submitted.

\section{S.02}

\section{Stromal and epithelial TGF- $\beta$ signaling in mammary} tumorigenesis

HL Moses, N Cheng, A Chytil, AE Gorska, M Aakre, E Forrester, EG Neilson, NA Bhowmick

Vanderbilt-Ingram Cancer Center, Department of Cancer Biology and Department of Medicine, Vanderbilt University Medical Center,

Nashville, Tennessee, USA

Breast Cancer Research 2005, 7(Suppl 2):S.02 (DOI 10.1186/bcr1045)

There is compelling evidence from transgenic mouse studies and analysis of mutations in human carcinomas indicating that the TGF- $\beta$ signal transduction pathway is tumor suppressive. We have shown that overexpression of TGF- $\beta 1$ in mammary epithelial cells suppresses the development of carcinomas and that expression of a dominant negative type II TGF- $\beta$ receptor (DNIIR) in mammary epithelial cells under control of the MMTV promoter/enhancer increases the incidence of mammary carcinomas. Studies of human tumors have demonstrated inactivating mutations in human tumors of genes encoding proteins involved in TGF- $\beta$ signal transduction, including DPC4/Smad4, Smad2, and the type II TGF- $\beta$ receptor (T $\beta R$ III). There is also evidence that TGF- $\beta$ can enhance the progression of tumors. This hypothesis is being tested in genetically modified mice. To attain complete loss of T $\beta$ RII, we have generated mice with loxP sites flanking exon 2 of Tgfbr2 and crossed them with mice expressing Cre recombinase under control of the MMTV promoter/enhancer to obtain Tgfbr2mgKO mice. These mice show lobuloalveolar hyperplasia. Mice are being followed for mammary tumor development. Tgfbr2mgKO mice that also express polyoma virus middle $T$ antigen under control of the MMTV promoter (MMTV-PyVmT) develop mammary tumors with a significantly shorter latency than MMTV-PyVmT mice and show a marked increase in pulmonary metastases. Our data do not support the hypothesis that TGF- $\beta$ signaling in mammary carcinoma cells is important for invasion and metastasis, at least in this model system.

The importance of stromal-epithelial interactions in mammary gland development and tumorigenesis is well established. These interactions probably involve autocrine and paracrine action of multiple growth factors, including members of the TGF- $\beta$ family, which are expressed in both stroma and epithelium. Again, to accomplish complete knockout of the type II TGF- $\beta$ receptor gene in mammary stromal cells, FSP1-Cre and Tgfbr2 $2^{\text {flox/llox }}$ mice were crossed to attain Tgfbr2 $2^{\text {fspKO }}$ mice. The loss of TGF- $\beta$ responsiveness in fibroblasts resulted in intraepithelial neoplasia in prostate and invasive squamous cell carcinoma of the forestomach with high penetrance by 6 weeks of age. Both epithelial lesions were associated with an increased abundance of stromal cells. Activation of paracrine hepatocyte growth factor (HGF) signaling was identified as one possible mechanism for stimulation of epithelial proliferation. TGF- $\beta$ signaling in fibroblasts thus modulates the growth and oncogenic potential of adjacent epithelia in selected tissues.

More recently, we have examined the effects of Tgfbr2 $2^{\text {fspKO }}$ fibroblasts on normal and transformed mammary epithelium. We analyzed the role of TGF- $\beta$ signaling by stromal cells in mammary tumor progression. To avoid the possibility of endogenous wild-type fibroblasts masking potential effects of Tgfbr2 ${ }^{\text {fspko }}$ cells on tumor progression, we implanted PyVmT mammary carcinoma cells with Tgfbr2 ${ }^{\text {fspKO }}$ or wildtype fibroblasts in the subrenal capsule of nude mice. Mammary tumor cells implanted with Tgfbr2 $2^{\text {fspKO }}$ cells exhibited an increase in tumor growth and intravasation associated with an increase in tumor cell survival, proliferation and an increase in tumor angiogenesis compared with tumor cells implanted with control fibroblasts. We demonstrated increased expression of several growth factors by Tgfbr2 $2^{\text {fspKO }}$ fibroblasts compared with control fibroblasts in primary culture. These included HGF, MSP and TGF- $\alpha$. There was an increase in tumor cell activating phosphorylation of the cognate receptors, c-Met, RON, erbB1, and erbB2 in carcinomas accompanied by Tgfbr2 ${ }^{\text {fspKO }}$ fibroblasts.

The Tgfbr2 ${ }^{\text {fspKO }}$ mouse model illustrates that a signaling pathway known to suppress cell-cycle progression when activated in epithelial cells can also have an indirect inhibitory effect on epithelial proliferation when activated in adjacent stromal fibroblasts in vivo. Loss of this inhibitory effect can result in increased epithelial proliferation and may even progress to invasive carcinoma in some tissues.

\subsection{3}

\section{Genomic analysis of human breast cancer in families and populations \\ M-C King}

University of Washington, USA

Breast Cancer Research 2005, 7(Suppl 2):S.03 (DOI 10.1186/bcr1046)

Abstract not submitted.

\section{S.04}

Abstract withdrawn.

\section{S.05}

ATM mutations associated with breast cancer RA Gatti ${ }^{1}$, P Concannon ${ }^{2}$

${ }^{1}$ UCLA School of Medicine, Department of Pathology and Laboratory Medicine, Los Angeles, California, USA; ${ }^{2}$ Benaroya Research Institute at Virginia Mason, Seattle, Washington, USA

Breast Cancer Research 2005, 7(Suppl 2):S.05 (DOI 10.1186/bcr1048)

Despite over a decade of scrutiny and over 20 published reports from various countries, the degree to which ATM mutations lead to breast 
cancer in the general population remains unclear. Furthermore, the methodology of ATM mutation detection is still laborious and costly. Because the ATM protein kinase phosphorylates such a wide array of downstream targets, many pathways to oncogenesis are possible and largely unexplored. What seems clear is that: A-T heterozygotes are at a fourfold to fivefold increased risk of breast cancer, although confidence intervals are large; and the spectrum of ATM mutations is distinct for A-T families versus breast cancer cohorts. Only a handful of mutations have been identified in both A-T families and breast cancer cohorts. Missense mutations represent $<10 \%$ of mutations in A-T patients and $>80 \%$ in breast cancer cohorts. ATM missense mutations are also more common in some leukemias and lymphomas. Experimental data suggest that some missense mutations represent dominant interfering mutations [1-5]; however, clinical support for a dominant interfering model is minimal in family studies, suggesting either that the model is flawed or that penetrance of these mutations is very low. Histological classifications of breast cancer are largely grouped as genetically homogeneous models, although expression microarray data suggest otherwise. Other studies have associated ATM-SNPs with increased breast cancer risk; however, just three SNP haplotypes across the ATM locus include $~ 95 \%$ of a global population, and this must be factored into such association models. Without the benefit of mRNA analyses, of minigene experiments, of Maximum Entropy Scores, of site-directed mutagenesis or of functional assays of ATM activity, most 'missense' mutations cannot be reliably distinguished from polymorphisms or from other types of mutations, such as splicing variants that lead to secondary stop codons. Our recent analyses have focused on two ATM missense mutations, 7271T $>$ G and IVS10-6T $>$ G. For each of these mutations, there are published functional data suggesting that they act as dominant interfering mutations, and epidemiological data suggesting a role in breast cancer. Some family studies of the $7271 \mathrm{~T}>\mathrm{G}$ mutation suggest that it is a highly penetrant breast cancer susceptibility allele. However, its infrequency in the population means that its contribution to breast cancer risk is slight and it is possible that $7271 \mathrm{~T}>\mathrm{G}$ represents only one of a diverse array of uncommon ATM mutations leading to increased cancer risk. We found that the frequency of the IVS10-6T>G mutation was not increased in breast cancer cases as compared with controls. Furthermore, the evidence that IVS10-6T>G is an A-T mutation is called into question by our recent evidence that, in the one known example of a homozygous IVS10-6T>G individual with A-T, a homozygous mutation at $5644 \mathrm{C}>\mathrm{T}$ was also present (Purayidom and colleagues, submitted). Taken together, these studies suggest that whereas no single ATM mutation impacts significantly upon breast cancer risk, it may be possible to group mutations that do modulate risk for breast cancer based on their phenotypic effects. This group of patients might benefit substantially from a therapeutic approach to correct missense mutations.

Acknowledgements These efforts were partially funded by NIH grant NS35322 and the A-T Medical Research Foundation, Los Angeles, California, USA.

References

1. Gatti RA, Tward A, Concannon P: Cancer risk in ATM heterozygotes: a model of phenotypic and mechanistic differences between missense and truncating mutations. Mol Biol Metab 1999, 68:419-423.

2. Spring K, Ahangari F, Scott SP, Waring P, Purdie DM, Chen PC, Hourigan $\mathrm{K}$, et al.: Mice heterozygous for mutation in Atm, the gene involved in ataxia-telangiectasia, have heightened susceptibility to cancer. Nat Genet 2002, 32:185-190.

3. Scott SP, Bendix R, Chen P, Clark R, Dork T, Lavin MF: Missense mutations but not allelic variants alter the function of ATM by dominant interference in patients with breast cancer. Proc Natl Acad Sci USA 2002, 99:925-930.

4. Concannon P: ATM heterozygosity and cancer risk. Nat Genet 2002, 32:89-90.

5. Chenevix-Trench G, Spurdle AB, Gatei M, Kelly H, Marsh A, Chen $\mathrm{X}$, Donn $\mathrm{K}$, et al.: Dominant negative ATM mutations in breast cancer families. J Nat/ Cancer Inst 2002, 94:205-215.

\section{S.06 \\ DNA damage response pathways in cancer causation and treatment MB Kastan, R Kitagawa, CJ Bakkenist}

Department of Hematology-Oncology, St Jude Children's Research Hospital, Memphis, Tennessee, USA

Breast Cancer Research 2005, 7(Suppl 2):S.06 (DOI 10.1186/bcr1049)

Cellular responses to DNA damage impact many aspects of cancer biology. First, damage to cellular DNA causes cancer. We know this from epidemiologic studies, from animal models, and from the observation that many human cancer susceptibility syndromes arise from mutations in genes involved in DNA damage responses. For example, the genes mutated in Fanconi's anemia, ataxia-telangiectasia, xeroderma pigmentosum, Li-Fraumeni syndrome, hereditary breast and ovarian cancers, and hereditary non-polyposis colon cancer are all involved in DNA damage responses. Second, DNA damage is used to cure cancer. The majority of the therapeutic modalities that we currently use to treat malignancies target the DNA, including radiation therapy and many chemotherapeutic agents. Third, DNA damage is responsible for the majority of the side effects of therapy. Bone marrow suppression, Gl toxicities, and hair loss are all attributable to DNA damage-induced cellular apoptosis of proliferating progenitor cells in these tissues. Thus, DNA damage causes the disease, is used to treat the disease, and is responsible for the toxicity of therapies for the disease. Significant progress has been made in recent years in elucidating the molecular controls of cellular responses to DNA damage in mammalian cells. These insights now provide us with approaches to attempt to manipulate these responses for patient benefit, such as enhanced tumor cell kill with therapy, protection of normal tissues from toxic effects of therapy, and even prevention of cancer development.

Many of the insights that we have gained into the mechanisms involved in cellular DNA damage response pathways have come from studies of human cancer susceptibility syndromes that are altered in DNA damage responses. One of these disorders, ataxia-telangiectasia (A-T), is characterized by multiple physiologic abnormalities, including neurodegeneration, immunologic abnormalities, cancer predisposition, sterility, and metabolic abnormalities. The gene mutated in this disorder, Atm, is a protein kinase that is activated by the introduction of DNA double-strand breaks in cells. Atm activity is required for cell cycle arrests induced by ionizing irradiation (IR) in G1, S, and G2 phases of the cell cycle. Several targets of the Atm kinase have been identified that participate in these IR-induced cell cycle arrests. For example, phosphorylation of $\mathrm{p} 53, \mathrm{mdm} 2$, and Chk2 participate in the G1 checkpoint; Nbs1, Brca1, FancD2, and Smc1 participate in the transient IR-induced S-phase arrest; and Brca1 and hRad17 have been implicated in the G2/M checkpoint. Although Atm is critical for cellular responses to IR, related kinases, such as Atr, appear to be important for responses to other cellular stresses [1]. Some substrates appear to be shared by the two kinases, with the major difference being which stimulus is present and which kinase is used to initiate the signaling pathway.

Characterization of these Atm substrates permitted us to manipulate these proteins in cell lines and to selectively abrogate single or multiple checkpoints. Using this approach, we demonstrated that abrogation of checkpoints does not by itself result in radiosensitivity. Although this has been known for several years in regards to the S-phase checkpoint, it was a surprising finding that abrogation of the G2/M checkpoint did not cause radiosensitivity. This observation suggested that some other function of Atm, other than checkpoint control, was important for cellular survival following ionizing irradiation. In characterizing targets of the Atm kinase, the only substrate whose phosphorylation seems to impact on radiosensitivity is Smc1 [2]. We previously demonstrated that the phosphorylation of Smc1 by ATM required the presence of both $\mathrm{Nbs} 1$ and Brca1 proteins. We recently found that this dependence results from the role that these two proteins play in recruiting both Smc1 protein and activated Atm to the sites of DNA breaks. We generated mice in which the two Atm 
phosphorylation sites in the Smc1 protein are mutated; cells from these mice demonstrate normal ATM activation, normal phosphorylation of both Nbs1 and Brca1 after IR, and normal migration of these proteins to DNA breaks [3]. Despite these normal activities of Atm, Nbs1 and Brca1, these cells exhibit a defective S-phase checkpoint, radiosensitivity, and increased chromosomal breakage after IR similar to that seen in cells lacking Atm. These results suggest that the phosphorylation of Smc1 is the critical target of this signaling pathway for these endpoints, and that the reason why cells lacking Nbs1 and Brca1 are radiosensitive and exhibit chromosomal breakage is due to a failure to recruit Smc1 to the sites of DNA breaks where it gets phosphorylated by previously activated Atm.

Recent studies also elucidated the mechanism by which DNA damage activates the Atm kinase and initiates these critical cellular signaling pathways [4]. Atm normally exists as an inactive homodimer bound to nuclear chromatin in unperturbed cells, and introduction of DNA damage induces intermolecular autophosphorylation on serine 1981 in both Atm molecules. This phosphorylation causes a dissociation of the Atm molecules and frees it up to now circulate around the cell and phosphorylate the substrates that regulate cell cycle progression and DNA repair processes. This regulation of Atm activity in the cell represents a novel mechanism of protein kinase regulation and appears to result from alterations in higher order chromatin structure rather than direct binding of Atm to DNA strand breaks. Although Nbs1 and Brca1 are not required for the initial activation of Atm after IR, these two proteins are required for the migration of activated Atm to the sites of DNA breaks. It is this process of recruitment of activated Atm along with Smc1 recruitment to the DNA breaks that leads to Smc1 phosphorylation by Atm and presumably initiation of some repair process(es) that reduce chromosomal breakage and enhance cell survival.

References

1. Bakkenist CJ, Kastan MB: Initiating cellular stress responses. Cell 2004, 118:9-17.

2. Kim S-T, Xu B, Kastan MB: Involvement of the cohesin protein, Smc1, in Atm-dependent and independent responses to DNA damage. Genes Dev 2002, 16:560-570.

3. Kitagawa R, Bakkenist CJ, McKinnon PJ, Kastan MB: Phosphorylation of SMC1 is a critical downstream event in the ATMNBS1-BRCA1 pathway. Genes Dev 2004, 18:1423-1438.

4. Bakkenist CJ, Kastan MB: DNA damage activates ATM through intermolecular autophosphorylation and dimer dissociation. Nature 2003, 421:499-506.

\section{S.07}

\section{SNPS in putative regulatory loci controlling gene expression in cancer VN Kristensen}

Department of Genetics, Institute for Cancer Research, The Norwegian Radium Hospital, Oslo, Norway

Breast Cancer Research 2005, 7(Suppl 2):S.07 (DOI 10.1186/bcr1050)

Given the increasing clinical importance of microarray expression classification of breast tumours and the different biology it may reveal [1], identifying an associated SNP profile may be of considerable value for pharmacogenetics, early diagnostics and cancer prevention. Studying the promoter composition of the genes that strongly predict the patient subgroups, we observed clear separation of the gene clusters based solely on their promoter composition, making feasible the hypothesis that SNPs in the regulatory regions of genes that create or abrogate transcription binding sites have the potential to influence the expression profiles. Morley and colleagues [2] reported linkage analysis of expression levels of 3554 genes and 2500 SNPs in 14 $\mathrm{CEPH}$ families (retrieved online [3]), and found significant evidence for the existence of regulation hot spots, suggesting both cis and trans regulatory effects. We report similar observations from a study with a different design, performing actual genotyping of 49 unrelated breast cancer patients, whose tumours have previously been analysed by genome-wide expression microarrays leading to a robust tumour classification with strong prognostic impact [4]. These patients were a part of a pharmacogenetic study of 193 patients who had received radiation therapy or chemotherapy. A high-throughput solid-phase, array-based method using primer extension chemistry has been used to perform the genotyping (GenomeLab ${ }^{\text {TM }}$ SNPstream genotyping system; Beckman Coulter, Fullerton, CA, USA). A total of 583 SNPs in 203 selected genes (1-19 SNPs/gene) were genotyped and tumour genome-wide expression was studied in 49 patients. Association in both cis and trans was detected for SNPs in 42 genes. SNPexpression associations with the top $0.25 \%$ best $P$ values $(9.81 \times$ $10^{-6}<P<0.001$ ) revealed regulatory SNPs in 115 genes in trans. The subsets of transcripts that were observed to have significantly many associations in common with a set of SNPs were further analysed using the gene ontology (GO) annotations. The GO terms of the unselected mRNA transcripts found associated to the SNPs in the selected candidate genes were often similar, suggesting that the observed associations are within the same functional pathway. Taken together these data suggest that the observed SNP-expression associations do exist and are observable even in a small set of unrelated individuals. A given expression profile of the tumour may be potentially associated and predicted by the genotype of the patient.

\section{References}

1. Perou CM, et al:: Nature 2000, 406:747-452.

2. Morley M, et al.: Nature 2004, 430:743-747.

3. The SNP Consortium Ltd [http://snp.cshl.org/]

4. Sørlie T, et al.: Proc Natl Acad Sci USA 2001, 98:10869-10874.

\subsection{8}

\section{Potential mechanisms whereby estrogens induce breast cancer in women RJ Santen, W Yue, J-P Wang}

University of Virginia Health Sciences System, Charlottesville, Virginia, USA

Breast Cancer Research 2005, 7(Suppl 2):S.08 (DOI 10.1186/bcr1051)

Long-term exposure to estradiol is associated with an increased risk of breast cancer in women. The data supporting this conclusion include: measurements of plasma total and free estradiol, estrone, and estrone sulfate and the aromatase substrate testosterone in postmenopausal women; the effect of oophorectomy before age 35; the effect of early menarche and late menopause; the relationship between bone density and breast cancer risk; and the role of menopausal hormone therapy on risk. However, the mechanisms responsible for estradiol-induced carcinogenesis are not firmly established. The prevailing theory postulates that estrogens increase the rate of cell proliferation by stimulating estrogen receptor (ER)-mediated transcription, thereby increasing the number of errors occurring during DNA replication. An alternative theory suggests that estradiol is metabolized to quinone derivatives, which directly remove base pairs from DNA through a process called depurination. Error-prone DNA repair then results in point mutations. We postulate that both processes act in an additive or synergistic fashion. If correct, aromatase inhibitors would block both processes, whereas anti-estrogens would only inhibit receptormediated effects. Our initial studies demonstrated that depurinating catechol-estrogen metabolites are formed in MCF-7 human breast cancer cells in culture. We then utilized an ERKO animal model that allows dissociation of ER-mediated function from the effects of estradiol metabolites, and demonstrated formation of genotoxic estradiol metabolites. We also examined the incidence of tumors formed in these ER $\alpha$ knockout mice bearing the Wnt-1 transgene. The absence of estradiol induced by castration markedly reduced the incidence of tumors and delayed their onset. Re-administration of estradiol to castrate animals induced tumors in a dose-responsive fashion. To ensure that all ER functionality was lacking, we administered fulvestrant and demonstrated that estrogen still induced breast tumors in these animals. On aggregate, our results support the concept that metabolites of estradiol may act in concert with ERmediated mechanisms to induce breast cancer. These findings support the possibility that aromatase inhibitors might be more effective than anti-estrogens in preventing breast cancer. Data from four clinical 
studies have now suggested that fewer contralateral breast cancers occur in women treated with aromatase inhibitors in the adjuvant setting than with tamoxifen. Taken together, our data provide experimental support for a genotoxic role for estradiol in hormonal carcinogenesis.

\section{S.09}

\section{The future of breast cancer prevention}

\section{A Howell, A Sims, M Harvie, KR Ong, G Evans, R Clarke}

CRUK Department of Medical Oncology, University of Manchester, Christie Hospital, Manchester, UK

Breast Cancer Research 2005, 7(Suppl 2):S.09 (DOI 10.1186/bcr1052)

At present, large numbers of at-risk women are treated in order to prevent relatively small numbers of breast cancers. There is a need to define risk more precisely in order to target interventions and a need to improve their efficacy. Risk estimations currently depend upon integration of familial and endocrine risk factors. We have demonstrated that the Tyrer-Cuzick model that takes both factors into account more fully is superior to other risk prediction models in our clinic [1]. However, prediction remains imprecise for the individual. Attempts are being made to take additional risk factors into account, including mammographic density [2], serum estradiol concentration and bone density. It seems probable that a better understanding of the interactions between stromal and epithelial cells in the breast including fibroblasts, adipocytes, macrophages and blood vessels will ultimately lead to better prediction. We have shown that $5 \%$ loss of body weight during mid life reduces postmenopausal breast cancer risk by $40 \%$ [3], and overviews indicate that use of NSAIDs [4] and exercise [5] may reduce risk by approximately $30 \%$. The mechanisms of these risk reductions are not clear but gene array studies indicate that calorie restriction and exercise predominantly reduce the expression of genes related to inflammation $[6,7]$. This raises the question of whether all these interventions act by similar mechanisms. A better understanding of the mechanisms of mammographic density and mammary cell senescence is required. Both are associated with fibroblasts that increase and stimulate proliferation of local epithelial cells $[8,9]$. Since mammographic density is a major risk factor, its reversal is likely to be beneficial. Another stromal target is aromatase. All adjuvant aromatase inhibitor (Al) trials have shown an approximately $50 \%$ contralateral breast cancer reduction compared with tamoxifen [10]. Since tamoxifen reduces contralateral risk by about $50 \%$ compared with placebo, Als may reduce risk by $70-80 \%$. Trials to test this hypothesis are underway (IBIS II, MAP3). The aforementioned considerations indicate that the stroma and stroma-epithelial interactions are already targets for preventive measures, and this is likely to expand and lead to new interventions such as NF- $\mathrm{kB}$ inhibition [11] and SIRT1 activation [12].

References

1. Amir E, et al:: J Med Genet 2003, 40:807.

2. Warwick J, et al:: Breast 2003, 12:10.

3. Harvie M, et al:: Cancer Epidemiol Biomarkers Prev 2005, 14:656-661.

4. Khuder SA, Mutgi AB: Br J Cancer 2001, 84:1188-1192.

5. Berglund G: IARC Sci Pub/ 2002, 156:237-241.

6. Clement $\mathrm{K}$, et al:: FASEB J 2004, 18:1658.

7. Bronikowski A, et al:: Physiol Genomics 2003, 12:129.

8. Tlsty T: Keystone Symposium, 5 February 2005.

9. Parinello S, et al.: J Cell Sci 2005, 118:485.

10. Howell A, et al:: Lancet 2005, 365:60.

11. Greten F, et al:: Cell 2004, 118:285.

\section{S.10}

\section{Targeting estrogen to kill ER-positive and ER-negative breast cancer vC Jordan}

Fox Chase Cancer Center, Philadelphia, Pennsylvania, USA

Breast Cancer Research 2005, 7(Suppl 2):S.10 (DOI 10.1186/bcr1053)

The current fashion of using long-term antihormonal therapies for the treatment and prevention of breast cancer has been remarkably successful over the past 20 years but this strategy has consequences for the development of drug resistance in remaining tumor tissue. Although estrogen is considered to be a survival signal that causes increased breast cancer cell replication, the study of drug resistance to antihormonal therapies has revealed an unanticipated new biology of estrogen action. Long-term antihormonal therapy eventually results in either tamoxifen or raloxifene (selective estrogen receptor modulators [SERMs]) stimulated growth and tumors are also stimulated to grow with estrogen. This is why aromatase inhibitors are effective treatments after the development of SERM resistance once the SERM is stopped. Long-term estrogen deprivation initially causes a cessation of breast tumor cell growth but eventually cells grow out that remain ER-positive but grow spontaneously. Estrogen deprivation with SERMs or aromatase inhibitors for more than 5 years causes a remarkable switching of the estrogen signaling pathway [1]. Instead of being a survival signal, physiologic concentrations of estrogen now cause apoptosis and tumor cell death. This knowledge provides an opportunity to test the hypothesis that low-dose estrogen therapy following exhaustive antihormonal therapy could be used as a successful treatment for patients. Studies are in place to evaluate the mechanism of action of estrogen-induced apoptosis so that a new target can be discovered to develop a novel apoptotic drug group. The ER-negative breast cancer cell is the ultimate hormone-resistant cell. Reintroduction of an active ER gene re-sensitizes the cells to estrogen that now causes blockade of the cell cycle [2] and apoptosis if cell survival signaling is also blocked. These data suggest that a universal target could be identified using the estrogen receptor mediated mechanism that will permit the broad application of new anti-apoptotic medicines.

References

1. Jordan VC: Selective estrogen receptor modulation: concept and consequences in cancer. Cancer Cell 2004, 5:207-213.

2. Jiang SY, Jordan VC: Growth regulation of estrogen receptornegative breast cancer cells transfected with complementary DNAs for estrogen receptor. J Natl Cancer Inst 1992, 84:580591.

\section{S.11}

\section{ER $\beta$ in normal and malignant breast J-Å Gustafsson, G Cheng, M Warner}

Department of BioSciences and Department of Medical Nutrition, Novum, Karolinska Institute, Huddinge, Sweden

Breast Cancer Research 2005, 7(Suppl 2):S.11 (DOI 10.1186/bcr1054)

Both ER $\alpha$ and ER $\beta$ are expressed in not only normal breast of the rodent, cow, monkey and human, but also in breast cancer. Cells that express ER $\alpha$ are found within the luminal epithelium, but not in the myoepithelium or stroma in the human breast. ER $\beta$, on the other hand, is expressed not only in the luminal epithelial cells, but also in myoepithelial cells, stromal cells and in passenger lymphocytes. This widespread distribution of ER $\beta$ suggests multiple roles for ER $\beta$ in the mammary gland.

We have shown that in the rodent mammary gland ER $\beta$ is the dominant $E R$, and that, in response to $E 2, E R \alpha$ but not $E R \beta$ is downregulated in the early $\mathrm{G} 1$ phase of the cell cycle. Cells that contain $\mathrm{ER} \alpha$ receive the signal to proliferate from E2, and within 4 hours of that signal $E R \alpha$ is lost from the nucleus. The cells then go through a complete cycle and $E R \alpha$ reappears in daughter cells. ER $\beta$ levels do not change in cell nuclei during the cell cycle. This pattern of ER regulation holds true in human breast cancer since $\mathrm{ER} \alpha$ is never co-localized with proliferation 
markers in breast cancer samples. This means that under the conditions of a constant high level of E2, ER $\alpha$ does not reappear in the nucleus. A similar situation exists during pregnancy when there is a constant high level of E2 and there is no ER $\alpha$ in the mammary epithelium. This resistance to the proliferative response to E2 in the presence of a constant high dose of E2 probably explains the very successful use of high-dose E2 in the treatment of breast cancer. ER $\beta$, on the other hand, appears to have a differentiative role not a proliferative role in the mammary gland, and the lactating rodent mammary gland of $\mathrm{ER} \beta^{-/-}$mice does not express gap junction and adhesion proteins, typical indicators of fully differentiated cells.

In recent years there have been several publications showing that ER $\beta$ is expressed in human breast cancer, and conclusions and speculations about a causative role for ER $\beta$ in breast cancer development and/or progression have been made. We have studied 500 frozen breast biopsies in collaboration with Prof. RC Coombes, London, in order to clarify the role of ER $\beta$ in normal and malignant breast. In this study we measured ER $\alpha$ and ER $\beta$ proteins by several techniques (immunohistochemistry, western blotting, ligand binding in sucrose gradients, and RT-PCR) in various human samples obtained from both benign breast and malignant breast. We found that ER $\beta$ is the predominant estrogen receptor in the normal mammary gland and in benign breast disease. There is very little $E R \alpha$ in the normal mammary gland. This low expression of $E R \alpha$ is one of the striking differences between rodents and humans. This is in stark contrast to ER $\beta$, which is expressed in $80 \%$ of epithelial cells and is also present in the stroma.

We found that ER $\alpha$ is abundantly expressed in invasive and in situ ductal carcinoma but not in medullary cancer. ER $\beta$ is also expressed in breast cancer, both ductal and medullary.

In this study we also found that, in the human breast, the major ER in breast stroma is ER $\beta$. This surprising finding has necessitated several new lines of investigation about the function of ER $\beta$ in the breast. It has long been thought that $E R \alpha$ in the stroma was responsible for secretion of growth factors in response to E2 and that these growth factors were responsible for epithelial cell proliferation. The discovery that it is $\operatorname{ER} \beta$ that is present in the stroma might suggest a role of ER $\beta$ in growth factor secretion.

\section{S.12}

\section{Molecular approaches to understanding pregnancy- induced protection against breast cancer CM Blakely, SE Moody, A Stoddard, E Tombler, C Liu,} LA Chodosh

Abramson Family Cancer Research Institute, University of Pennsylvania School of Medicine, Philadelphia, Pennsylvania, USA Breast Cancer Research 2005, 7(Suppl 2):S.12 (DOI 10.1186/bcr1055)

The marked protection against breast cancer afforded women by an early first full-term pregnancy has important clinical implications for designing chemopreventive approaches to breast cancer and, more generally, for understanding how cancer susceptibility can be modulated by normal developmental events. Epidemiologic studies have repeatedly demonstrated that women who undergo an early first full-term pregnancy have a significantly reduced lifetime risk of breast cancer. Similarly, rodents that have previously undergone a full-term pregnancy are highly resistant to carcinogen-induced breast cancer compared with age-matched nulliparous controls. Relatively little progress has been made, however, towards understanding the molecular basis of this phenomenon. We have used microarray expression profiling to identify persistent changes in gene expression in the mouse and rat mammary gland that are induced by an early first fullterm pregnancy. Using this approach, we have isolated a panel of genes whose expression is persistently altered in multiple strains of mice and rats by a reproductive event known to reduce breast cancer risk. Additional studies are underway to compare gene expression patterns in mammary tissues from parous and nulliparous mice, rats, and women with parity-induced changes in gene expression that are evolutionarily conserved. Similarly, gene expression patterns in rats that have been treated with hormonal regimens that mimic parity-induced protection are being compared with those induced by non-protective control regimens in order to identify genes whose expression patterns are most closely correlated with protection. Finally, gene expression changes induced by parity in strains of rats that exhibit different levels of susceptibility to carcinogen-induced tumorigenesis are being compared. These gene expression changes suggest novel hypotheses for the mechanisms by which parity may modulate breast cancer risk and will be useful for probing the mechanisms by which the developmental state of the mammary gland modulates the response to an oncogenic stimulus.

\subsection{3}

\section{Predicting response/resistance to endocrine therapy for breast cancer} WR Miller', TJ Anderson', D Evans'2, A Krause'2, JM Dixon'1

${ }^{1}$ Breast Unit, University of Edinburgh, Western General Hospital, Edinburgh, UK; ${ }^{2}$ Novartis Pharma AG, Femara GBTR-Research, Basel, Switzerland

Breast Cancer Research 2005, 7(Suppl 2):S.13 (DOI 10.1186/bcr1056)

Background Endocrine therapy for breast cancer is a major modality for the treatment of breast cancer, producing response rates between $30 \%$ and $40 \%$ of unselected patients with the minimum of toxicity. However, the majority of patients receive no benefits and, after successful treatment, tumour regrowth may occur. Optimal management therefore requires accurate predictors of response and early identification of resistance. The present article reviews results from neoadjuvant studies in which endocrine therapy was given to patients whose primary breast cancer was still within the breast so that changes in tumour volume could be used to assess clinical response and so that sequential biopsies could be taken for molecular analyses designed to identify predictive markers.

Methods All patients had histologically confirmed breast cancer and were treated for 3-4 months with either tamoxifen or an aromatase inhibitor (anastrozole, exemestane or letrozole). Core or excisional tumour biopsies were taken before and at the end of treatment (and at $10-14$ days in certain studies). Oestrogen receptors (ER), progestogen receptors and c-erbB1 and c-erbB2 were measured by immunohistochemistry. Microarray analysis was performed on tumour RNA extracted and amplified before hybridization on Affymetrix HG_U133A GeneChips for microarray analysis.

Results Steroid hormone receptor status highly influences the response to all endocrine therapies, negative tumours failing to respond and response being more likely with increasing levels of ER and the concomitant presence of PgR. Conversely, tumour overexpression of c-erbB2 (and c-erbB1) is associated with resistance to tamoxifen but not aromatase inhibitors. While these receptors are helpful in identifying groups of tumours with differing sensitivity to endocrine therapy, they fail to predict accurately in individual cases. To address this deficiency, in Edinburgh we have looked for early genetic changes (at 10-14 days) that occur with treatment and might be associated with subsequent response to the aromatase inhibitor letrozole. Clinical response data were available for 43 cases, of which $33(77 \%)$ were classified as responders $(>50 \%$ reduction in tumour volume) and $30(70 \%)$ displayed evidence of pathological response. No gene changed substantially with treatment in all cases; however, there was consistent upregulation of three genes and downregulation of 65 genes in 50 of the cases. Based on clustering techniques, it was possible to identify highly consistent changes in gene expression with treatment, which allowed tumours to be subdivided into groups showing distinct patterns of molecular changes. While the change in expression of any single gene failed to correlate with response, significant differences in change of expression in 125 genes were detected between non-responders and responders. A combination of gene changes produced increased discrimination. The identity of the genes and their relevance to the prediction of response and mechanisms of resistance will be discussed. 
Conclusions Early changes in gene expression profiles may define tumour groups with differing sensitivity to endocrine therapy and permit early recognition of response and resistance. However, clinical utility at the level of individual patients has yet to be validated and explored.

\section{S.14}

\section{Genetic and epigenetic changes in early carcinogenesis \\ TD TIsty}

Department of Pathology and UCSF Comprehensive Cancer Center, University of California at San Francisco, California, USA

Breast Cancer Research 2005, 7(Suppl 2):S.14 (DOI 10.1186/bcr1057)

Studies of human epithelial cells and fibroblasts from healthy individuals are providing novel insights into how early epigenetic and genetic events affect genomic integrity and fuel carcinogenesis. Key epigenetic changes, such as the hypermethylation of the $\mathrm{p} 16$ promoter sequences, create a previously unappreciated pre-clonal phase of tumorigenesis in which a subpopulation of epithelial cells is positioned for progression to malignancy [1]. These key changes precede the clonal outgrowth of premalignant lesions and occur frequently in healthy, disease-free individuals [2]. Prior work from our laboratory has shown that surrounding stroma can dramatically influence tumorigenesis. Proper stromal-epithelial interactions can actually suppress the expression of preneoplastic phenotypes in epithelial cells and, conversely, altered stromal-epithelial interactions can promote the probability that preneoplastic lesions progress to malignancy [3]. Understanding more about these early events should provide novel molecular candidates for prevention and therapy of cancer.

\section{References}

1. Nature 2001, 409:636.

2. Cancer Cell 2004, 5:263.

3. Cancer Res 1999, 61:5002.

\section{S.15}

\section{A breast cancer progression model: the importance of three-dimensional tissue architecture and metalloproteinases \\ MJ Bissell, C Myers, G Lee, E Lee, A Rizki, S Mian, J Gray, D Radisky}

Life Sciences Division, Lawrence Berkeley National Laboratory, Berkeley, California, USA

Breast Cancer Research 2005, 7(Suppl 2):S.15 (DOI 10.1186/bcr1058)

Previous studies from our laboratory have shown that non-malignant and malignant cells can be distinguished easily and rapidly by their morphology and growth rate when cultured in three-dimensional (3D) laminin-rich basement membrane but not when cultured on traditional tissue culture plastic (two-dimensional [2D]) [1,2]. In addition, we have shown that cellular responses to signaling inhibitors and apoptotic agents differ in cells cultured in 2D versus 3D $[3,4]$. This applies also to our finding with reverted tumor cell lines [3-8]. In this presentation, I will address two inter-related topics.

First, we asked how the 3D morphology and gene expression profiles for a panel of 60 breast cancer cell lines for which the Gray laboratory has obtained 2D expression as well as CGH profiles may differ, and whether any of the surrogate genes or phenotypes could track with response to therapy. The cell lines examined so far fell into four distinct morphologies of 'round', 'mass', 'grape-like' and 'stellate'. An ANOVA analysis of Affymetrix gene expression profiles for each of these cell lines was used to identify genes, the expression profiles of which could distinguish the other known parameters of the cultured cells. Of the 22,283 genes on the Affymetrix 133A chip, 25800 genes were identified where expression patterns differed between different cell lines both in 2D and 3D, and $\sim 2000$ genes were identified where expression differed between the non-malignant and malignant cell lines. About 700 genes differed between 2D and 3D, and $\sim 800$ correlated with the morphological differences seen in 3D. These genes fall into a number of functional classes, which we are currently analyzing to identify common signaling themes and/or morphological regulators that will be tested by manipulation of expression and correlated with therapeutic response of these cell lines in 2D and 3D to Herceptin and other chemotherapeutic drugs.

Second, we have also shown previously that loss of basement membrane in both cultured mammary mouse cells [9] and in transgenic animals led to epithelial to mesenchymal transition (EMT) and mammary tumors [10]. We have now determined the molecular pathways induced by MMP-3 to lead to EMT and genomic instability via production of reactive oxygen species [11]. These mechanisms will be discussed.

References

1. Petersen OW, Ronnov-Jessen L, Howlett AR, Bissell MJ: Proc Natl Acad Sci USA 1992, 89:9064-9068.

2. Schmeichel KL, Bissell MJ: J Cell Sci 2003, 116:2377-2388.

3. Wang F, et al:: Proc Natl Acad Sci USA 1998, 95:14821-14826.

4. Weaver VM, et al.: Cancer Cell 2002, 2:205-216.

5. Weaver VM, et al:: [cover feature] J Cell Biol 1997, 137:231-246.

6. Wang F, et al.: J Natl Cancer Inst 2002, 94:1494-1503.

7. Liu H, et al.: J Cell Biol 2004, 164:603-612.

8. Bissell MJ, Rizki A, Mian IS: Curr Opin Cell Bio/ 2003, 6:753-762.

9. Lochter A, et al.: J Cell Biol 1997, 139:1861-1872.

10. Sternlicht MD, et al:: Cell 1999, 98:137-146.

11. Radisky DC, et al.: Nature 2005 , in press.

\section{S.16}

Genomic and transcriptional events associated with poor clinical responses to conventional therapies K Chin 1,2, S Devries', J Fridlyand2, P Spellman', W-L Kuo',2, A Lapuk ${ }^{1,2}$, R Neve', T Tokuyasu², C Kingsley², S Dairkee ${ }^{3}$, K Chew ${ }^{2}$, A Jain ${ }^{2}$, BM Ljung², L Esserman², F Waldman², JW Gray ${ }^{1,2}$

${ }^{1}$ Lawrence Berkeley National Laboratory, Berkeley, California, USA; 2University of California at San Francisco, California, USA; ${ }^{3}$ California Pacific Medical Center, San Francisco, California, USA

Breast Cancer Research 2005, 7(Suppl 2):S.16 (DOI 10.1186/bcr1059)

Advances on several fronts have led to increases in survival duration and to reduced mortality in patients with breast cancer. These include improved procedures for earlier detection, optimization of combined surgical and radiotherapy, and use of optimized selective estrogen receptor modifiers (SERMS) and new chemotherapeutic strategies including gene-targeted therapies. In addition, molecular stratification strategies have been developed that stratify patients according to outcome. Stratification based on measurement of expression 'signatures' have been particularly effective and seem likely to improve treatment strategies. Patients at increased risk of progressive disease can be offered standard of care chemotherapy. However, some of these patients do not respond well to these treatments and current stratification strategies provide little information to guide treatment of these patients. This study of tumors from patients treated according to standard of care identifies genomic and coordinated transcriptional aberrations - especially amplification at $11 q$, and $20 q$ in tumors with the luminal $A$ expression phenotype, and at $17 q$ in tumors associated with the ERBB2 expression phenotype - that are strongly associated with poor response to such treatment. Our study identifies genes in these regions of amplification that can be assessed to identify patients that will respond poorly to the current standard of care and that are targets for therapies that will be effective against these poorly responding tumors. Interesting, this study also shows that patients with basal-like tumors do not have substantially shorter survival durations than patients with luminal-like tumors, suggesting that basal-like tumors respond well to the adjuvant adriamycin and cyclophosphamide therapies employed during their treatment. 


\section{S.17}

\section{The role of the tumor microenvironment in breast} cancer progression

\section{H Min, J Yao, M Allinen, L Cai, K Polyak}

Dana-Farber Cancer Institute and Harvard Medical School, Boston, Massachusetts, USA

Breast Cancer Research 2005, 7(Suppl 2):S.17 (DOI 10.1186/bcr1060)

We performed comprehensive molecular analysis of each cell type composing normal breast tissue and in situ and invasive breast carcinomas. Gene expression profiles were analyzed using serial analysis of gene expression, genetic changes were analyzed by single nucleotide polymorphism arrays, while epigenetic changes were analyzed using methylation-specific digital karyotyping. Based on these data we determined that gene expression and epigenetic changes occur in all cell types during breast cancer progression, while genetic alterations were only detected in tumor epithelial cells. Many of the differentially expressed genes encode for secreted proteins and receptors suggesting alterations in autocrine and paracrine interactions in breast tumorigenesis. Two of these genes, the CXCL14 and CXCL12 chemokines, overexpressed in tumor myoepithelial cells and in myofibroblasts, respectively, bind to receptors on epithelial cells and enhance their proliferation, migration, and invasion. Chemokines may thus play a role in breast tumorigenesis by acting as paracrine factors. The role of these chemokines, and myoepithelial and stromal cells in the progression of in situ carcinomas to invasive carcinomas was investigated using a xenograft model of human ductal carcinoma in situ. Based on our studies we determined that changes in the tumor microenvironment and epithelial-myoepithelial and epithelial-stromal cell interactions play an important role in breast tumor progression.

Reference

1. Allinen M, Beroukhim R, Cai, L, Brennan C, Lahti-Domenici J, Huang H, Porter D, Hu M, Chin L, Richardson A, et al.: Molecular characterization of the tumor microenvironment in breast cancer. Cancer Cell 2004, 6:17-32.

\section{S.18}

\section{Biological features and xenograft models of a very early human premalignant breast lesion S Lee, Y Wu, SK Mohsin, D Medina, DC Allred}

Breast Center, Baylor College of Medicine, Houston, Texas, USA Breast Cancer Research 2005, 7(Suppl 2):S.18 (DOI 10.1186/bcr1061)

Background Most breast cancers appear to arise from certain precursors over long periods of time. Enlargement $(>50$-fold) of normal terminal duct lobular units (TDLUs) by hyperplastic epithelial cells is one of the most common and earliest histologically recognizable alterations with premalignant potential. Understanding how these hyperplastic enlarged lobular units (HELUs) develop and progress could lead to new and effective strategies for breast cancer prevention therapy.

Methods The estrogen receptor (ER) and proliferation (Ki67) were evaluated and compared in TDLUs and HELUs in the same breasts $(n=250)$ by immunohistochemistry. Apoptosis was also assessed by the TUNEL assay. The rate of ER expression in proliferating cells was assessed by dual-labeled immunofluorescence. Comprehensive gene expression profiling was performed in a subset of samples (currently six matched pairs of TDLUs and HELUs) using RNA isolated from microdissected formalin-fixed paraffin-embedded breast tissue samples and Affymetrix U133-X3P microarrays analyzed by dCHIP software. Xenografts of human TDLUs and HELUs were prepared by implanting isolated epithelial cells into cleared mammary fat pads of estrogenstimulated immune-compromised mice 'humanized' by prior local injection of immortalized (h-tert transfected) human mammary fibroblasts.

Results The average ER expression was significantly elevated in HELUs compared with adjacent TDLUs ( $85 \%$ vs $30 \%$ positive cells, respectively; $P<0.0001)$. Proliferation was significantly higher $(6 \%$ vs $2 \% ; P<0.0001)$ and apoptosis was significantly lower $(0.6 \%$ vs $0.2 \% ; P<0.001)$ in HELUs than TDLUs. There was a large increase in the proportion of ER-positive proliferating cells in HELUs compared with TDLUs (35\% vs $4 \% ; P<0.0001)$. In preliminary analysis of the microarray results, HELUs and TDLUs segregated perfectly in unsupervised hierarchical comparisons. In supervised comparisons, many $(n=74)$ genes showed $>3$-fold $(P<0.05)$ differences in expression, with 45 relatively up (from 3.5 -fold to 9.5 -fold) and 29 relatively down (from 3.5-fold to 12.5 -fold) in HELUs versus TDLUs. Especially prominent elevations in HELUs included several genes involved in G-protein signaling, the retinoic acid pathway, and detoxification. Prominent decreases included genes involved in cell cycle inhibition, apoptosis, differentiation, and water transport. Differences were also noted in the expression of genes for ligands of the epidermal growth factor receptor between HELUs and TDLUs. Several fresh human samples of TDLUs and HELUs are in various stages (currently up to generation four) of implantation in mice in attempts to establish stable xenografts.

Conclusions HELUs are one of the earliest histologically recognizable lesions in the human breast with premalignant potential. They show striking elevations of ER, which may partially explain the hyperplasia leading to their development from TDLUs through increased proliferation and decreased apoptosis, which are both regulated by estrogen. DNA microarrays reveal many additional differences in the expression of genes involved in growth and differentiation. Human xenograft models are under development to support mechanistic studies of these genes to understand their roles in the development and progression of HELUs and how to prevent it.

Acknowledgments This work was supported by funds from the Astra Zeneca/Baylor College of Medicine Research Alliance and $\mathrm{NIH} / \mathrm{NCl}$ grant U01-CA84243.

\section{S.19}

\section{Regulation of epithelial cell polarity during carcinogenesis} SK Muthuswamy

Cold Spring Harbor Laboratory, Cold Spring Harbor, New York, USA Breast Cancer Research 2005, 7(Suppl 2):S.19 (DOI 10.1186/bcr1062)

Pathogenesis of cancer begins as hyperplastic lesions; some lesions remain benign, while others progress to malignancy. An increase in cell proliferation rates and changes in tissue architecture are two properties commonly observed in hyperplastic lesions. A great deal is known about the molecular events that regulate cell proliferation and the knowledge gained is widely used for development of diagnostic and treatment tools. Our understanding of the mechanisms that deregulate tissue architecture is poor, and hence it is understandable that the use of architectural features to determine prognosis of early lesions has varying success. We used polarized epithelial cells and an inducible method of ErbB2 activation to investigate whether the cell architecture influences ErbB2-induced gene expression and to investigate how activation of ErbB2 disrupts epithelial cell architecture. Activation of ErbB2 in three-dimensional epithelial acini-like structures leads to expression of a unique set of genes that was not observed when ErbB2 was activated in cells grown on plastic dishes, suggesting that the cell architecture can have significant influence on ErbB2-induced gene expression. To investigate the effect of ErbB2 activation on epithelial architecture, we activated ErbB2 in polarized epithelial cells. ErbB2 induced a loss in apical-basal polarity, re-initiated proliferation and induced multilayering of epithelial sheets. These changes correlate with the ability of ErbB2 to regulate the Par complex, a protein complex known to regulate establishment of epithelial cell polarity. Inactivation of atypical protein kinase $\mathrm{C}$, a component of the Par complex, cooperates with ErbB2 to disrupt polarized epithelial cells, suggesting that the Par complex is a mediator of ErbB2-induced effects on polarized epithelial cells. In addition, we identify tricellular junctions, and not bicellular junctions, as a novel site for ErbB2 action in cultured epithelial cells and in primary breast cancer. We are thus beginning to gain novel insights into the molecular mechanisms that regulate early lesions. 


\section{S.20 \\ Expression profiling of peripheral blood cells for early detection \\ A-L Børresen-Dale', P Sharma ${ }^{2}$}

${ }^{1}$ Department of Genetics, Institute for Cancer Research, The Norwegian Radium Hospital, Oslo, Norway; ${ }^{2}$ DiaGenic ASA, Oslo, Norway

Breast Cancer Research 2005, 7(Suppl 2):S.20 (DOI 10.1186/bcr1063)

Existing methods to detect breast cancer in asymptomatic patients have limitations, and there is a need to develop more accurate and convenient methods. Especially, an accurate method for breast cancer detection based on peripheral blood as a clinical sample will be highly desirable because of the easy accessibility and less-invasive nature by which samples can be obtained.

Results demonstrating that peripheral blood can be used to develop a gene expression based test for early detection of breast cancer will be presented. The rationale for using blood cells as monitors for a malignant disease elsewhere in the body is based on the hypothesis that a malignant growth will cause characteristic changes in the biochemical environment of blood. These changes will affect the expression pattern of certain genes in blood cells.

We initially conducted a pilot study where the expression pattern of 1368 genes in peripheral blood cells of 24 females with breast cancer and 32 females with no signs of this disease were analyzed using macroarrays and the expression data analyzed by PAM. The results were validated using a standard leave-one-out cross-validation approach. We were able to identify a set of genes that correctly predicted the diagnostic class in at least $82 \%$ of the samples. The majority of the identified genes had a decreased expression in samples from breast cancer patients, and predominantly encoded proteins implicated in ribosome production and translation control. In contrast, the expression of some defence-related genes was increased in samples from breast cancer patients.

In order to revalidate these findings and to increase the repertoire of informative genes, we have now extended the study with a larger number of breast cancer and non-breast cancer samples and used Agilent WG oligo arrays for large-scale gene expression analysis. The preliminary analysis of the data supports our previous finding that a blood-based gene expression test can potentially be developed to detect breast cancer in asymptomatic patients.

Reference

1. Sharma P, Sahni NS, Tibshirani R, Skaane P, Urdal P, Berghagen $H$, Jensen M, Kristiansen L, Moen $C$, Sharma $P$, et al.: Early detection of breast cancer based on gene expression patterns in peripheral blood cells. Breast Cancer Res 2005, in press.

\section{S.21}

\section{Stem cells in human breast development and cancer M Wicha, G Dontu, S Liu, I Mantle}

University of Michigan Comprehensive Cancer Center, Ann Arbor, Michigan, USA

Breast Cancer Research 2005, 7(Suppl 2):S.21 (DOI 10.1186/bcr1064)

The epithelial components of the breast are thought to arise from a stem cell population that is capable of both self-renewal and lineagespecific differentiation. We and others have hypothesized that mammary stem cells or their immediate progeny are targets for transformation during carcinogenesis. Normal stem cells and carcinoma cells share many characteristics including self-renewal capacity, telomerase expression, ability to differentiate, resistance to apoptosis, and ability to home to specific sites. Mammary transformation may require dysregulation of pathways that control normal stem cell selfrenewal such as Notch, Wnt, Hedgehog, and Bmi-1. In order to study these pathways in normal mammary development, we have developed an in vitro culture system in which primary human epithelial cells isolated from reduction mammoplasties are cultured as 'mammospheres' on non-adherent surfaces. Cells within mammospheres are able to self-renew, as well as to differentiate into all the lineages found in the mammary gland. Utilizing this system, we demonstrate bi- directional interaction between Notch and Hedgehog signaling and Bmi-1 in the regulation of stem cell self-renewal. When mammospheres are admixed with irradiated human mammary fibroblasts and implanted into the cleared fatpads of NOD/SCID mice, they are able to reconstitute the ductal alveolar structures found in the human mammary gland.

The stem cell model of carcinogenesis may also provide a partial explanation for the generation of cellular heterogeneity seen within mammary tumors. Using flow cytometry, we have identified a small population of cells within primary or metastatic breast cancers that bear the cell surface phenotype ESA ${ }^{+} \mathrm{CD} 44^{+} \mathrm{CD} 24^{-/ \text {low }}$ Lineage $^{-}$that have the properties of human tumor stem cells. As few as 200 of these cells are able to reproducibly generate tumors in NOD/SCID mice, while the vast majority of cells in these tumors that lack this phenotype are incapable of tumor formation even when tens of thousands of cells are injected. Consistent with a stem cell model, tumorigenic cells generate tumors that recapitulate the phenotypic heterogeneity found in the original tumors. We have demonstrated that pathways that control normal stem cell self-renewal, such as Hedgehog, are activated in mammary tumor stem cells, compared with their differentiated progeny. Despite progress in breast cancer therapeutics, metastatic breast cancer remains an incurable disease. Current therapies that have been developed by virtue of their ability to induce tumor regression may selectively target more differentiated cells in tumors, while leaving the tumor stem cell population intact, accounting for treatment resistance and relapse. Multiple mechanisms may account for this resistance to apoptosis, including increased expression of antiapoptotic genes, increased DNA repair mechanisms, and transporter proteins such as BCRP found in the tumor stem cell population. The targeting of stem cell self-renewal pathways such as Hedgehog or Notch may thus provide a novel and more effective approach for the treatment of advanced breast cancer.

\section{S.22}

\section{Molecular distinctions among ERBB2-overexpressing breast cancers \\ SS Jeffrey}

Stanford University, Stanford, California, USA

Breast Cancer Research 2005, 7(Suppl 2):S.22 (DOI 10.1186/bcr1065)

HER2 or c-ERBB2/neu is a member of the epidermal growth factor receptor (EGFR) family and encodes a tyrosine kinase receptor. Overexpression of HER2 protein is generally attributable to gene amplification. HER2 is overexpressed in $20-30 \%$ of primary invasive breast carcinomas and in a greater proportion of in situ breast cancers. Invasive breast cancers that overexpress HER2 are generally higher stage, show lymph node positivity, and have higher S-phase. Moreover, they are often associated with poor prognosis, particularly in node-positive patients.

Microarray studies have subdivided breast cancers into several subtypes. HER2-overexpressing ER-negative tumors are generally classified within a single subtype denoted ERBB2-overexpressing. However, ERpositive HER2-overexpressing tumors are usually intermixed with other ER-positive tumors that do not show HER2 overexpression.

Our recent population-based study evaluating HER2 overexpression and hormone receptor status has unexpectedly found that the majority of HER2-overexpressing tumors are hormone receptor-positive and are more common than HER2-overexpressing ER-negative breast cancers. This implies that the ERBB2-overexpressing molecular subtype, which is associated with ER-negative status, only includes a minority of HER2-overexpressing tumors. We therefore studied gene expression patterns of HER2-overexpressing breast cancers and found several tumor subtypes with distinctive molecular signatures. These ERBB2overexpressing subtypes spanned the range of hormone receptor status and highlighted different biological characteristics. Since the clinical course varies among patients with HER2-positive tumors, as does their response to targeted therapy, differences in global gene expression among HER2-overexpressing tumors could be important in distinguishing patients for the design and delivery of individualized targeted therapies. 


\section{S.23}

\section{Insulin-like growth factor regulation of mammary gland development and tumorigenesis \\ AV Lee}

Breast Center, Baylor College of Medicine and the Methodist Hospital, Houston, Texas, USA

Breast Cancer Research 2005, 7(Suppl 2):S.23 (DOI 10.1186/bcr1066)

Insulin-like growth factors (IGFs) are potent mitogens and survival factors. In the mammary gland, IGFs stimulate proliferation, differentiation, and survival during numerous developmental stages; IGF signaling is required for puberty-dependent ductal outgrowth, stimulates lobuloalveolar development during pregnancy, and is reduced or absent during apoptosis-driven involution. Much of our knowledge of IGF action in the mammary gland in vivo comes from knockout or transgenic models. However, very few of these studies have examined the consequence of these gene alterations on IGF signaling in vivo. We have recently shown that intravenous injection of IGF-I stimulates IGF-IR and IRS phosphorylation in the mammary gland, and we are currently assessing the effect of targeted gene deletion of overexpression of IGF signaling components on downstream signaling in the mammary gland in vivo.

Many years of research have shown that the proliferative and survival functions of the IGFs are not only important in mammary gland development, but are also strongly involved in mammary cancer. Early work using breast cancer cell lines in vitro showed that IGFs could increase cell growth and survival; in particular, that IGFs could block the effects of chemotherapy. We have recently shown that breast cancer cell lines grown as xenografts in vivo are also sensitive to intravenous IGF stimulation, and several IGF-IR inhibitors have been shown in the past year to block MCF-7 xenograft growth.

IGF-IR and its downstream signaling intermediate IRS- 1 can transform fibroblasts in vitro. To date there is no evidence for their transforming ability in vivo using transgenic mice. We have recently characterized mice that overexpress IGF-IR, IRS-1, or IRS-2 in the mammary gland, using mouse mammary tumor virus directed overexpression. We have found that overexpression of a constitutively active IGF-IR in the mammary gland disrupts normal development, such that female mice are unable to lactate, and that mice rapidly develop mammary tumors. Interestingly, overexpression of IRS-1 or IRS-2 also causes mammary tumorigenesis, albeit with a longer time to tumor formation than dominant active IGF-IR. These are the first mouse models showing that IGF-IR or IRS overexpression leads to tumorigenesis in vivo. We are currently examining the pathways required for IGF-IR and IRS-mediated tumorigenesis in the mammary gland.

\section{S.24}

\section{Targeting the cell cycle for prognosis and therapy of breast cancer \\ K Keyomarsi, S Akli}

University of Texas, MD Anderson Cancer Center, Houston, Texas, USA Breast Cancer Research 2005, 7(Suppl 2):S.24 (DOI 10.1186/bcr1067) Cyclin $E$ is a $G_{1}$-cyclin that plays a key role in the $G_{1}$ to $S$ transition of the cell cycle. Cyclin $E$ is processed in tumor cells by an elastase-like protease into low-molecular-weight (LMW) isoforms that are biochemically hyperactive. The LMW isoforms of cyclin $\mathrm{E}$ are unique to cancer cells. In breast cancer, such alteration of cyclin $E$ is a very strong predictor of poor patient outcome.

Alterations in the binding properties of these LMW isoforms to CDK2 and the CDK inhibitors (CKIs), p21 and p27, result in their functional hyperactivity. The LMW forms of cyclin $\mathrm{E}$ are several-fold more effective at binding to CDK2. Additionally, compared with the full-length cyclin E-CDK2 complexes, the LMW cyclin E-CDK2 complexes are significantly more resistant to inhibition by $\mathrm{p} 21$ and $\mathrm{p} 27$, despite equal binding of the CKIs to the LMW complexes. When both the full-length and the LMW cyclin E are co-expressed, p27 preferentially binds to the LMW forms yet is unable to inhibit the CDK2 activity. When overexpressed in breast cancer cells, the LMW forms of cyclin E, but not the full-length form, result in their hyperactivity due to increased affinity for cdk2 and resistance to inhibition by the CDK inhibitors p21 and p27, result in resistance to the growth inhibiting effects of antiestrogens, and result in chromosomal instability. Finally, tumors from breast cancer patients overexpressing the LMW forms of cyclin E are polyploid in nature and are resistant to endocrine therapy.

To assess the oncogenic role of cyclin E-LMW as compared with fulllength cyclin $E$, we examined the consequences of overexpressing these isoforms in the mammary glands of transgenic mice using the MMTV promoter. Four constructs were generated: MMTV-M46A coding for the full-length cyclin E (EL1), MMTV-EL1/EL4 coding for EL1 and the isoform translated at methionine 46 (EL4), and MMTV-T1 and MMTV-T2 coding for the isoforms generated by elastase cleavage at the first site (EL2 + EL3) and at the second site (EL5 and EL6), respectively. For each construct at least two transgenic lines were established. Transgene expression was demonstrated by RT-PCR, northern blotting and western blotting. Overexpression of cyclin E was seen in more than $90 \%$ of ductal and lobular cells of the mammary glands for each independent line. Mammary-specific LMW cyclin E overexpression induced extensive abnormalities at 2 months, including perturbed architecture, polyploidy, anysocytosis and apoptosis. Wholemount preparations of mammary glands at different development stages showed that overexpression of EL1/EL4 and cyclin E-T1 induced growth delay, while at 6 months of age an increased proportion of cells in the $S$ phase was found $(25.6 \pm 5.6 \%$ for EL1/EL4, $9.0 \pm 2.7 \%$ for T1 compared with $3.9 \pm 1.9 \%$ for nontransgenic animals). We observed a $34 \%$ (13/38) incidence of mammary adenocarcinomas in the EL1/EL4 transgenic lines with a mean latency of 18.3 months, and observed a $20 \%(5 / 25)$ incidence in the $\mathrm{T} 1$ transgenic lines with a mean latency of 17.1 months. The tumor incidence rate of the other transgenic lines, M46A and T2, are still unknown due to the young age of the mice (all under 7 months of age) and the long latency of cyclin E-mediated tumor generation. Thirty percent (4/13) of the EL1/EL4 and 40\% (2/5) of the T1 tumor-bearing animals developed lung metastasis. The tumors induced by the EL1/EL4 and T1 transgenes were mainly solid adenocarcinomas with very little differential to glandular for EL1/EL4 and mostly glandular for T1. Since p53 alterations are common in human breast carcinomas, we bred a $\mathrm{T} 1$ line with $\mathrm{p} 53^{+/-}$mice. The $\mathrm{T} 1 \times \mathrm{p} 53^{+/-}$cross generated tumors that are much more malignant than the T1 tumors; the incidence increased to $100 \%$, with a much shorter latency of 11 months. Biochemical analysis of the tumors revealed that $64 \%$ (9/14) retained cyclin $E$ expression and that, on average, the cyclin E-overexpressing tumors had threefold higher cyclin E kinase activity than the non-cyclin E-expressing tumors. Taken together, these data indicate that tumor progression in cyclin $E$ transgenic mice follow sequential steps of dysplasia, mammary intraepithelial neoplasia and invasive/metastatic tumors.

Collectively, the biochemical and biological differences between the full-length and the LMW isoforms of cyclin E provide a molecular mechanism for the poor clinical outcome observed in breast cancer patients harboring tumors expressing high levels of the LMW forms of cyclin E. The transgenic mouse model system can serve as a useful system in which to study the mechanisms responsible for LMW cyclin E-induced genetic instability and may help identify those factors that promote tumor progression and metastasis. The properties of the LMW forms of cyclin E suggest that they are not just surrogate markers of poor outcome, but that they are bona fide mediators of aggressive disease and potential therapeutic targets for patients whose tumors overexpress these forms.

\section{S.25}

\section{Apoptotic chemotherapies} AB Pardee, PV Reddy, DK Biswas, CJ Li

Dana-Farber Cancer Institute, Boston, Massachusetts, USA

Breast Cancer Research 2005, 7(Suppl 2):S.25 (DOI 10.1186/bcr1068)

Mutations derange growth regulations of cancer cells. They can also make the cells more subject to apoptosis. We have investigated two 
drugs that produce specific apoptotic chemotherapeutic mechanisms that cause tumor shrinkage in mice without deleterious side effects. The small natural product b-lapachone is specifically apoptotic to a variety of cancer cells. It synergizes strongly with taxol. It seems to have several mechanisms of lethality depending on the tumor type and the drug concentration. One mechanism is to elevate the major S-phase transcription factor E2F-1, to an apoptotic concentration [1]. It is now in clinical trial.

Tumor cells often mutate to apoptosis resistance; for example, by inactivating the p53 protein. We have reported that Go6976, a kinase inhibitory small molecule, can decrease activation of the anti-apoptotic transcription factor NF- $\mathrm{KB}$ [2].

These two novel therapies thus specifically cause cancer cell apoptosis; one by increasing an apoptotic factor, and the other restoring apoptosis by decreasing an anti-apoptotic factor.

References

1. Li Y, Sun X, LaMont T, Pardee AB, Li C: Selective killing of cancer cells by b-lapachone: direct checkpoint activation as a strategy against cancer. Proc Natl Acad Sci USA 2003, 100: 2674-2678.

2. Biswas DK, Martin KJ, McAllister C, Cruz AP, Graner E, Dai S, Pardee AB: Apoptosis caused by chemotherapeutical inhibition of nuclear factor-kB activation. Cancer Res 2003, 63:290-295.

\section{S.26 \\ High-resolution representational oligonucleotide microarray analysis and fluorescence in situ hybridization analysis of aneuploid and diploid breast tumors \\ J Hicks', V Grubor'1, N Navin', P Lundin², S Månér², T Hägerström ${ }^{2}$, L Skoog ${ }^{2}$, M Wigler', A Zetterberg ${ }^{2}$}

${ }^{1}$ Cold Spring Harbor Laboratory, Cold Spring Harbor, New York, USA; ${ }^{2}$ Cancer Center Karolinska, Karolinska Institute, Stockholm, Sweden

Breast Cancer Research 2005, 7(Suppl 2):S.26 (DOI 10.1186/bcr1069)

Background Combining representational oligonucleotide microarray analysis (ROMA) of tumor DNA with quantitative multigene fluorescence in situ hybridization (QM-FISH) of individual tumor cells provides the opportunity to detect and validate a wide range of gene amplifications, deletions, duplications and rearrangements directly in frozen tumor samples.

Methods We have used these combined techniques to examine 101 aneuploid and diploid breast tumors (highly aneuploid A-tumors and pseudo-diploid D-tumors), for which long-term follow-up and detailed clinical information were available.

Results We have determined that ROMA provides accurate and sensitive detection of duplications, amplifications and deletions, and it yields defined boundaries for these events with a resolution of less than $50 \mathrm{kbp}$ in most cases.

Conclusion Diploid tumors are particularly useful subjects for this approach, revealing complex rearrangements and repeated sequential amplification events on certain chromosomes that provide unique insights into the genomic progression of the disease. First, the fine structure of these amplification clusters, as detected by ROMA and quantitatively validated by $\mathrm{FISH}$, provides extremely high-resolution 'pointers' to potential novel oncogenes, since many of the detected amplicons contain only one or two known or prospective genes. Second, FISH patterns provide a means for interpretation of the mechanism of these events. Third, the reproducibility and frequency of these events, especially in very early stage tumors, provides insight into the earliest chromosomal events in breast cancer. Finally, we have identified correlations between certain sets of rearrangement events and clinically relevant parameters such as long-term survival. These correlations may enable novel and powerful prognostic indicators for breast cancer and other cancers when more samples can be examined.

\subsection{7 \\ Tailored therapies based upon tumor subtype biology CM Perou}

Department of Genetics and Department of Pathology, Lineberger Comprehensive Cancer Center, The University of North Carolina at Chapel Hill, North Carolina, USA

Breast Cancer Research 2005, 7(Suppl 2):S.27 (DOI 10.1186/bcr1070)

Breast cancer is a spectrum of diseases comprised of different tumor subtypes, each with a distinct biology and clinical behavior. To capture this diversify, we characterized the variation in gene expression across human breast tumors using DNA microarrays and identified at least five distinct tumor subtypes that are statistically significant predictors of patient overall survival [1]. Recently, we further validated these findings using a training set of 102 tumors, which was used to derive a new 'intrinsic gene set'. This gene set was then validated using a true test set of 311 tumors compiled from three different microarray studies. Our analyses demonstrate that common patterns of gene expression can be identified across different microarray platforms, that the breast tumor 'intrinsic' subtypes are reproducible across different datasets, and that this classification was a significant predictor of outcomes after correcting for standard clinical parameters such as estrogen receptor (ER), grade and node status [2].

The biology of the 'intrinsic' subtypes is rich and extensive, and many of these expression features suggest distinct therapies. The 'intrinsic' subtypes include at least two types of ER-negative tumors (Basal-like and $\mathrm{HER}^{+} / \mathrm{ER}^{-}$) and at least two types of ER-positive tumors (Luminal A and Luminal B). Basal-like tumors typically show low expression of HER2 and ER, and these tumors exhibit high expression of genes characteristic of the basal epithelial cell layer, including expression of keratin 5, keratin 6, keratin 17 and four Kallikrein genes (KLK5-KLK8). The Basal-like tumors pose a challenge from the treatment perspective because they lack ER and HER2. However, we have recently shown that most are HER1-positive and/or c-KIT-positive [3], and we have initiated a clinical trial to evaluate the efficacy of HER1-inhibitors in preselected Basal-like tumor patients.

HER2-positive (i.e. gene amplified) tumors fall into at least two distinct expression groups: those that are ER-negative and typically cluster near the Basal-like tumors (HER2 $\left.{ }^{+} / \mathrm{ER}^{-}\right)$, and those that are ER-positive and cluster with tumors of luminal cell origin. These findings suggest that both types of HER2 ${ }^{+}$patients should receive transtuzumab, but that the $\mathrm{ER}^{+} / \mathrm{HER} 2^{+}$may gain a benefit from hormone therapy.

Finally, the Luminal subtype A and Luminal subtype B tumors express ER, GATA3, and genes regulated by both ER and GATA3. Compared with Luminal $B$ tumors, Luminal A tumors express higher levels of ER, BCL2 and GATA3, and they show more favorable patient outcomes. Luminal B tumors more often express HER1, HER2 and/or cyclin $E_{1}$, and they show worse outcomes. Our data, when coupled with data from others [4], suggests that Luminal A patients are likely to benefit from hormone therapy and are not likely to benefit from chemotherapy, while the opposite may be true of Luminal B patients. Experiments to answer these questions in Luminal patients are underway and will be discussed. References

1. Sørlie T, Tibshirani R, Parker J, Hastie T, Marron JS, Nobel A, Deng S, Johnsen H, Pesich R, Geisler S, et al.: Repeated observation of breast tumor subtypes in independent gene expression data sets. Proc Natl Acad Sci USA 2003, 100:8418-8423.

2. Hu Z, Fan C, Marron JS, He X, Qaqish BF, Karaca G, Livasy C, Carey L, Reynolds E, Dressler L, et al.: The molecular portraits of breast tumors are conserved across microarray platforms. 2005, submitted.

3. Nielsen TO, Hsu FD, Jensen K, Cheang M, Karaca G, Hu Z, Hernandez-Boussard T, Livasy C, Cowan D, Dressler L, et al.: Immunohistochemical and clinical characterization of the basal-like subtype of invasive breast carcinoma. Clin Cancer Res 2004, 10:5367-5374.

4. Paik S, Shak S, Tang G, Kim C, Baker J, Cronin M, Baehner FL, Walker MG, Watson D, Park T, et al.: A multigene assay to predict recurrence of tamoxifen-treated, node-negative breast cancer. N Engl J Med 2004, 351:2817-2826. 


\subsection{8}

\section{Expression profiling as a prognostic and predictive factor in breast cancer}

\section{UJ van 't Veer}

The Netherlands Cancer Institute, Amsterdam, The Netherlands Breast Cancer Research 2005, 7(Suppl 2):S.28 (DOI 10.1186/bcr1071)

Microarray gene expression profiling combined with advanced bioinformatics is beginning to show its power in delineating disease entities that are otherwise indistinguishable. This refinement in tumor classification allows a more accurate prediction of outcome of disease for patients that present with the same stage of disease based on conventional clinical and histopathological criteria. Gene activities determining the biological behaviour of the tumor may indeed be more likely to reflect the aggressiveness of the tumor than general parameters such as tumor size, age of the patient, or even tumor grade. The immediate clinical consequences are therefore that treatment schemes can be tailored based on the gene activity patterns of the primary tumor.

Using gene expression profiling with cDNA microarrays, Perou and colleagues showed that there are several subgroups of breast cancer patients based on unsupervised cluster analysis: those of 'basal type' and those of 'luminal type'. These subgroups differ with respect to outcome of disease in patients with locally advanced breast cancer. In addition, microarray analysis has been used to identify diagnostic categories (e.g. BRCA1 and BRCA2, estrogen receptor status).

We used gene expression profiling with DNA microarrays harboring 25,000 genes on 78 primary breast cancers of young lymph-nodenegative patients to establish a signature, predictive for a short interval to distant metastases. This 'poor prognosis' signature consists of genes involved in the cell cycle, invasion and angiogenesis. The prognosis signature is superior to currently available clinical and histopathological prognostic factors in predicting a short interval to distant metastases (odds ratio $=18$ [95\% confidence interval $=$ 3.3-94], $P<0.001$, multivariate analysis). We have validated our findings of this poor prognosis profile on a large unselected consecutive series of $\mathrm{LNO}$ as well as lymph-node-positive $(\mathrm{LN}+)$ young breast cancer patients $(n=295)$. The analyses confirm that the profile is a strong independent factor in predicting outcome of disease for LNO patients in general (10-year overall survival for the good prognosis profile $96 \%$ vs $50 \%$ for the poor prognosis profile). Furthermore, the profile is also powerful for $\mathrm{LN}+$ patients. At present, the prognostic significance of the 70 genes is tested in older breast cancer patients.

Nowadays, consensus guidelines in the management of breast cancer select up to $95 \%$ of lymph-node-negative young breast cancer patients for adjuvant systemic therapy (e.g. $\mathrm{NIH}$ and St Gallen consensus criteria). As $70-80 \%$ of these patients would have remained diseasefree without this adjuvant treatment, these patients are 'overtreated'. The 'poor prognosis' signature provides a novel strategy to accurately select patients who would benefit from adjuvant systemic therapy and can greatly reduce the number of patients that receive unnecessary treatment.

Our data revealed that already small tumors display the metastatic signature, and recent results show that the molecular program established in a primary breast carcinoma is highly preserved in its distant metastasis. These findings suggest that metastatic capability in breast cancer is an inherent feature, and is not based on clonal selections. The results further imply that neo-adjuvant treatment given to patients based on (yet to be established) response expression profiles of their primary breast tumor might indeed prevent the outgrowth of micrometastases.

Currently, the EORTC breast group is preparing a 5000-patient randomized trial to compare the efficacy of guidance of breast cancer patients for adjuvant chemotherapy based on either 'conventional' St Gallen consensus criteria or the microarray prognosis test (MINDACT trial within the EU-TRANSBIG program). The aim of the study is to confirm that the microarray test will save up to $30 \%$ of the patients from unnecessary chemotherapy and to identify $5 \%$ of them who are nowadays 'undertreated'.

\section{S.29 \\ Genomic profiling of breast cancer Å Borg}

Lund University, Sweden

Breast Cancer Research 2005, 7(Suppl 2):S.29 (DOI 10.1186/bcr1072)

Cancer and other genetic diseases are characterized by genome alterations, including DNA copy number changes. Comparative genomic hybridization (CGH) represents a powerful technique to detect and map these aberrations, and recent improvements in resolution and sensitivity have been possible through implementation of microarray-based platforms. Germline mutations in the two major breast cancer susceptibility genes, BRCA1 and BRCA2, account for a significant proportion of all hereditary breast cancers. Earlier studies have shown that inherited and sporadic tumors progress along different somatic genetic pathways and that global gene expression profiles distinguish between these groups. Using $1 \mathrm{Mbp}$ resolution BAC-array CGH analysis, we now show that genomic copy number profiles similarly discriminate between BRCA1/BRCA2-related tumors and sporadic tumors. Overall, BRCA1 tumors had a higher frequency of copy number alterations than sporadic breast cancers. In particular, frequent losses on $4 p, 4 q$ and $5 q$ in BRCA1 tumors and frequent gains on $7 p$ and $17 q 24$ in BRCA2 tumors distinguish these from sporadic breast cancer. Distinct amplicons at 3q27.1-q27.3 were identified in BRCA1 tumors, and amplicons at 17q23.3-q24.2 in BRCA2 tumors. Moreover, evidence of a homozygous deletion in a BRCA1 tumor on $5 q 12.1$ was obtained. Using a set of 169 BAC clones that detect significantly different frequencies of copy number changes in inherited and sporadic tumors, these subsets could be discriminated into separate groups using hierarchical clustering. Further validation may prove this tumor classifier to be useful for selecting familial breast cancer cases, likely to carry BRCA1 or BRCA2 germline mutations, for further mutation screening, particularly as these data can be obtained using DNA prepared from archival tumor tissue.

Further improved genomic profiling was obtained by construction of microarrays comprising 32,433 BAC clones, offering complete genome coverage at single gene resolution, on average $<50 \mathrm{kbp}$. These new tiling $32 k$-arrays were evaluated on breast cancer cell lines (BT-474, MCF7, HCC1937, SK-BR-3, L56Br, ZR-75-1), validated by $\mathrm{FISH}$ and gene expression analysis. Known amplicons were resolved and found to include complex patterns of narrow peaks, occasionally including a few or even single genes. Several amplified regions and genes on $17 q$ and $20 q$ were depicted and confirmed by demonstrating strong correlations between gene copy numbers and expression. Previously described as well as novel homozygous deletions, ranging from a few BAC clones $(<300 \mathrm{~kb})$ to several Mbp, were observed, including PTEN and other regions on $10 \mathrm{q}, \mathrm{CDH} 1 / \mathrm{CDH} 3$ on $16 \mathrm{q} 22$, and new regions on $4 q 34$ and $19 p 12$, emphasizing the power of array $\mathrm{CGH}$ in pinpointing genes of importance in tumor development. Array $\mathrm{CGH}$ is a promising diagnostic tool in profiling of somatic and constitutional genomic alterations.

\section{S.30}

\section{A single nucleotide polymorphism in the HDM-2 gene regulates the p53 apoptotic response and influences the age of onset of cancers in humans: the SNP 309 HDM-2 polymorphism \\ GL Bond', AJ Levine',}

${ }^{1}$ Cancer Institute of New Jersey, New Brunswick, New Jersey, USA; 2Institute for Advanced Study, School of Natural Sciences, Princeton, New Jersey, USA

Breast Cancer Research 2005, 7(Suppl 2):S.30 (DOI 10.1186/bcr1073)

The HDM-2 gene in humans has two promoters for transcription. $5^{\prime}$ to the first exon is a maintenance promoter providing low levels of HDM-2 in the cell. In the first intron are the P53 DNA binding sites and the p53 inducible promoter that yields threefold to 10-fold more HDM-2 mRNA after a p53 activation and response. When this intronic promoter is employed, transcriptional initiation starts at the second exon and this 
mRNA is translated more efficiently than mRNA that starts at the first exon. The coding region of the HDM-2 protein starts in the third exon. At residue 309 in this first intron is a single nucleotide polymorphism, with $12 \%$ of people being a G/G homozygote, $40 \%$ being a G/T heterozygote and $48 \%$ of people being $T / T$ wild-type homozygotes (the $\mathrm{G} / \mathrm{G}$ genotype is lower in black Americans and the sample size is now over 300 people). We have found that the $G / G$ genotype creates a better SP-1 transcription factor binding site, raises the level of $m$-RNA in unstressed cells and produces threefold to sixfold more HDM-2 protein in cells (cancer cells in culture) with the $\mathrm{G} / \mathrm{G}$ genotype. This mRNA starts at the second exon, and is probably translated better in unstressed cells. After DNA damage or other stresses, P53 activity in cells with the G/G genotype is lower and the percentage of cells undergoing apoptosis is lower when compared with cells in culture with $T / T$ genotypes. We have reproduced these observations with lymphocytes taken from human volunteers and placed in culture, with EBV-immortalized B cells in culture, with primary fibroblasts in cell culture and with cancer cell lines in culture. In 92 individuals that have donated lymphocytes we see individuals forming a distribution of apoptotic responses between $20 \%$ and $60 \%$ after gamma radiation, with individuals being quite reproducible in repeated experiments. The lower half of the distribution is heavily weighted with the G/G genotype, while the upper half of the distribution has mainly the $T / T$ genotype. The higher HDM-2 levels in cells thus result in a lower apoptotic index in cells from these volunteers. It has become clear in recent studies that SNP 309 has a clinical impact. We have genotyped two cancer cohorts, one at MD Anderson and one in Germany, containing patients with sarcomas and breast cancers. The results have been statistically significant $(P=0.01-0.02)$ and clear in both cohorts, and the average age of onset of these cancers is $10-15$ years earlier in people with the G/G genotype than in people with the same cancer with the $T / T$ genotype. The interpretation is then that the probability of eliminating pre-cancerous clones of cells via a p53 mechanism is lower in people with a G/G genotype (high HDM-2 levels) and the probability of developing a cancer at an earlier time in life is higher. In addition, in patients that have a germline mutation in the p53 gene (this yields one-half of the p53 protein level in a cell) those individuals that have a $G / G$ genotype or a $G / T$ genotype develop multiple cancers (three, four or five cancers) over their lifetimes, while no T/T homozygotes develop that many independent cancers.

\section{S.31}

Evading p53 action during tumor development and
therapy
sW Lowe

Cold Spring Harbor Laboratory, Cold Spring Harbor, New York, USA Breast Cancer Research 2005, 7(Suppl 2):S.31 (DOI 10.1186/bcr1074)

Apoptosis is a regulated form of cell death that is important for normal development and tissue homeostasis. Senescence produces 'genetic death', in that the senescent cell is incapable of further propagation. Both processes are frequently disrupted in cancer cells, and each act as potent barriers to tumorigenesis. Since radiation and many chemotherapeutic agents induce apoptosis or senescence, the integrity of these programs can influence the outcome of cancer therapy. Our laboratory strives to understand how cancer genes control apoptosis and senescence in normal cells, and how mutations that disrupt these processes impact tumor development and therapy. The goal of these efforts is develop therapeutic strategies based on an understanding of drug action and cancer genotype. We currently are using genetically engineered mouse models to understand how apoptosis and senescence are controlled in tumor cells, as well as the response of tumors to conventional and targeted therapeutics. Recent work exploring the action of tumor-derived myc mutants in oncogenesis and the role of the p53 tumor suppressor network in the action of targeted therapeutics will be discussed.

\subsection{2 \\ TP53 and additional pathways in therapy resistance PE Lønning}

Haukeland University Hospital, Bergen, Norway

Breast Cancer Research 2005, 7(Suppl 2):S.32 (DOI 10.1186/bcr1075)

Resistance to chemotherapy is the main obstacle to cancer cure. Despite encouraging results from preclinical studies, we have limited knowledge regarding mechanisms causing therapy resistance in vivo. We previously identified mutations affecting the L2 and/or L3 domains of the TP53 gene to predict resistance to anthracycline as well as mitomycin therapy [1,2]. However, while TP53 mutations were significantly associated with therapy failure, we observed tumours resistant to therapy despite harbouring wild-type p53. We also saw responding tumours among those harbouring TP53 mutations affecting the L2 or L3 domains.

Based on these assumptions, we postulated that chemoresistance could be due to failure of the 'p53 pathway' acting in concert with one, or more, redundant pathways [3]. In a recent paper we thus reported a mutation of the CHEK2 gene among one of the tumours resistant to therapy despite harbouring wild-type TP53 [4]. In addition, we are searching for redundant pathways that may compensate for the p53 mechanism. Strikingly, looking at genetic alterations associated with resistance to other drugs with respect to other malignancies, this seems to concentrate on drugs known to be involved in so-called 'family cancer syndromes', meaning genes involved either in growth arrest, apoptosis or DNA damage repair [3]. This may seem logical, as much of the damage created by chemotherapeutic drugs resemble genetic events involved in carcinogenesis. Thus, at this stage, our interest is focused on genetic pathways involving genes involved in 'family cancer syndromes'. An update of our current results will be presented.

References

1. Aas T, et al:: Specific P53 mutations are associated with de novo resistance to doxorubicin in breast cancer patients. Nat Med 1996, 2:811-814.

2. Geisler S, et al.: TP53 gene mutations predict the response to neoadjuvant treatment with FUMI in locally advanced breast cancer. Clin Cancer Res 2003, 9:5582-5588.

3. Lønning PE: Genes causing inherited cancer as beacons identifying the mechanisms of chemoresistance. Trends Mol Med 2004, 10:113-118.

4. Staalesen $\mathrm{V}$, et al.: Alternative splicing and mutation status of CHEK2 in stage III breast cancer. Oncogene 2004, 23:85358544.

\section{S.33 \\ Dynamic imaging of plasticity and escape in tumor cell invasion \\ P Friedl}

Rudolf Virchow Center for Experimental Biomedicine and Department of Dermatology, University of Würzburg, Germany

Breast Cancer Research 2005, 7(Suppl 2):S.33 (DOI 10.1186/bcr1076)

Cancer cell interactions with the extracellular matrix and the migration therein require adhesion and traction provided by integrins, together with pericellular proteolysis executed by extracellular matrix degrading proteases. We have used experimental interference strategies and identified plasticity of migration modes resulting in new ways of dissemination. As imaged by three-dimensional matrix-based models and intravital microscopy, quantitative reconstruction from movies has shown how tumor cells depend on adhesion mechanisms but continue to migrate after adhesion receptors are blocked, has shown how proteases generate proteolytic tracks but are dispensable if 'physical' strategies allow cells to bypass tissue barriers, and has shown why individual and collective invasion patters predispose to a different outcome after pharmacotherapeutic intervention.

These findings have implications with reference to invasion as a therapeutic target in progressive cancer disease. 


\section{S.34 \\ Role of HER2 in local relapse and metastasis S Ménard}

Molecular Targeting Unit, National Cancer Institute, Milan, Italy Breast Cancer Research 2005, 7(Suppl 2):S.34 (DOI 10.1186/bcr1077)

HER2-positive breast carcinomas have been shown to display an early peak of relapses in the first 4 years after surgery, especially in the node-positive subgroup. To explain this observation, growth factors released at the time of surgery were investigated. The level of growth factors of the EGF family, detected in postsurgical sera from breast carcinoma patients, was found to correlate with surgical invasiveness. Indeed, following radical mastectomy, higher levels of serum EGF-like factors were found than after conservative surgery. This implicates that the growth of tumors overexpressing HER2, activated by these growth factors, should be stimulated after invasive surgery. Two retrospective analyses of the HER2 status of primary tumors included in a randomized clinical trial addressing the issue of conservative versus invasive surgery and of radiotherapy were performed by immunohistochemistry using the standardized herceptest. Survival analysis according to surgery indicated no differences in HER2-negative cases but indicated a poorer survival for HER2-positive node-positive patients who had mastectomy in comparison with those who had conservative surgery. Furthermore, local relapses in patients who had conservative surgery without radiotherapy were found to be anticipated in the HER2-positive subset. This is a 'proof of principle' that surgery by inducing growth factor release may be detrimental for patients with HER2-positive tumors. To verify these findings, a prospective analysis of the follow-up of more than $\mathbf{2 0 0 0}$ patients who have had mastectomy or conservative surgery is ongoing. Preliminary data indicate a significantly worst prognosis of HER2-positive tumors after invasive surgery, above all for tumors scoring $2+$ by immunohistochemistry. Acknowledgement Partially supported by the AIRC.

\subsection{5}

\section{Molecular profiling of early breast cancer in relation} to detection of micrometastases and outcome

\section{B Naume'1, T Sørlie ${ }^{2}$}

${ }^{1}$ Department of Oncology and 2 Department of Genetics, The Norwegian Radium Hospital, Oslo, Norway

Breast Cancer Research 2005, 7(Suppl 2):S.35 (DOI 10.1186/bcr1078)

Background Molecular profiling of breast cancer by DNA microarrays has been used to classify tumors into five distinct subclasses that show significant differences in clinical outcomes. Of these subclasses, the luminal subtype $A$ is associated with a relatively good prognosis [1-3]. Detection of disseminated tumor cells (DTC) in bone marrow (BM) can independently predict future metastasis, which was confirmed in our study of 817 early BrCa patients [4].

Materials and methods Fresh tumor samples were prospectively collected during primary surgery from 123 of these patients, for evaluation of the clinical significance of gene expression profiling and for comparison of tumor subtypes with DTC detection in BM. The BM samples were collected from iliac crests at primary surgery, followed by immunocytochemical staining (anti-cytokeratin mAbs) and morphologyguided screening for DTC. Gene expression patterns of the primary tumors were examined using 42,000 spot cDNA microarrays (Stanford Functional Genomics Facility). Data were analyzed by hierarchical clustering and were compared with our previously published breast tumor subclassifications. Data were further analyzed by supervised analysis methods (SAM, PAM).

Results The tumors were classified by gene expression analysis into luminal A (41\%), luminal B (13\%), ERBB2+ (17\%), basal-like (14\%) and normal-like (12\%). The luminal A subtype showed high ER/PgRpositivity (98\%), low ERBB2-positivity (4\%) (assessed by IHC) and low frequency of TP53 mutations (6\%). Luminal B, ERBB2 ${ }^{+}$and basal-like subtypes showed high frequencies of TP53 mutations (43\%,65\%, and $82 \%$, respectively), whereas the ER/PgR-positivity was $94 \%, 24 \%$ and $6 \%$, respectively. Expression of the ERBB2 protein differed between these groups. At median 60 months follow-up, luminal A patients showed improved survival compared with patients within the other subtypes $(P=0.02$, log rank), with $\mathrm{BrCa}$ death in $14 \%$ versus $29 \%$, respectively. DTC in BM were detected in 23.7\%. No particular subtype was associated with DTC, and no particular gene profile was associated with DTC status, as determined by SAM analysis. However, when we stratified the patients based on the molecular subtype, and first considered only the luminal A tumors, we identified 193 genes (FDR 23\%) associated with high expression in tumors from patients with DTC. Moreover, a considerable number of patients with a luminal A type of tumor experienced systemic relapse of the disease $(28 \%)$ and SAM analysis identified 147 genes associated with different expression patterns in tumors from relapsed patients versus diseasefree patients

Conclusion This early BrCa study confirms the consistency of the gene expression profiles and their clinical implications. DTC detection can further distinguish the clinical outcome in patients with the luminal A subtype. The gene expression patterns in DTC-positive patients, and in all patients with systemic relapse, will be further explored.

References

1. Perou CM, Sørlie T, Eisen MB, et al:: Nature 2000, 406:747-752.

2. Sørlie T, Perou CM, Tibshirani R, et al.: Proc Natl Acad Sci USA 2001, 98:10869-10874.

3. Sørlie T, Tibshiranhi R, Parker J, et al.: Proc Natl Acad Sci USA 2003, 100:8418-8423.

4. Wiedswang, Borgen, Kåresen, et al:: J Clin Oncol 2003, 21: 3469-3478.

\section{S.36}

\section{Update on HER2-directed therapy \\ D Slamon}

University of California, Los Angeles, California, USA

Breast Cancer Research 2005, 7(Suppl 2):S.36 (DOI 10.1186/bcr1079)

Abstract not submitted.

\section{S.37}

\section{Targeting new therapies in combination with hormonal therapies for ER-positive breast cancer M Dowsett}

Academic Department of Biochemistry, Royal Marsden Hospital, London, UK

Breast Cancer Research 2005, 7(Suppl 2):S.37 (DOI 10.1186/bcr1080)

Hormonal therapies involving estrogen deprivation or SERMs such as tamoxifen reduce the risk of relapse and improve the survival of the $>75 \%$ of breast cancer patients with ER-positive tumours. Nonetheless, many of these patients relapse with disease that was either intrinsically resistant to treatment or that has acquired resistance to the endocrine treatment. Laboratory studies have revealed that growth factor receptor pathways form an important route of growth signalling in both these circumstances, and there is now a series of agents available that target these pathways at different points. This provides the opportunity to utilise these agents in combination with endocrine treatment and the possibility that this may extend the effectiveness of the hormonal agents. The effective delivery of such combinations depends on a detailed knowledge of the degree to which the highly encouraging laboratory findings are translated into the clinical scenario. We have demonstrated that almost all breast ERpositive cancer shows some proliferative dependence on oestrogen, but that this is very variable. We have begun to identify in clinical samples the key genes whose expression both determines this variability and are themselves dependent on it. The development of novel models of drug development that allow the assessment of the expression of these genes, particularly within the presurgical setting, offers major opportunities to assess the potential of the various new targeted agents to be combined with endocrine therapy. 


\section{S.38 \\ Development of CDK inhibitors as cancer therapeutics \\ D Lane \\ IMCB, Singapore}

Breast Cancer Research 2005, 7(Suppl 2):S.38 (DOI 10.1186/bcr1081)

The cyclin-dependent kinases form a large family of enzymes in human cells that are involved in the control of cell proliferation and transcription. A large number of small molecule inhibitors of this class of enzyme have been developed in both the pharmaceutical and academic communities, and at least two have entered clinical trial, having shown efficacy in preclinical models. Alterations in the activity of this class of enzymes is a frequent feature of human cancers, brought about by altered expression of either the enzymes themselves or their regulators such as $\mathrm{p} 21, \mathrm{p} 27$ and $\mathrm{p} 16$. The exact role of each of the different kinases has proved hard to determine as knockout mouse studies have implied a degree of redundancy and the exact substrates of each enzyme in vivo are still unclear. In addition, most of the current inhibitors are not specific to a single form of the enzyme and new regulatory pathways are still being discovered. Intense studies of one such inhibitor, R-Roscovitine (CYC202), including trials involving more than 100 patients, have established the potential of the class as nongenotoxic anti-cancer drugs. In some model systems the activity of this class of compound is best explained by their activity as inhibitors of transcriptional elongation, and a link between this mechanism and the induction of apoptosis has been established. The concept of cyclinspecific inhibitors as more sophisticated genetic models of target validation in this field will be discussed.

\section{S.39 \\ Genes, genomes, and cancer \\ D Botstein}

Princeton University, New Jersey, USA

Breast Cancer Research 2005, 7(Suppl 2):S.39 (DOI 10.1186/bcr1082) Abstract not submitted.

\section{S.40}

\section{The search for low-penetrance breast cancer genes BAJ Ponder, A Cebrian, AM Dunning, DF Easton, F Lesueur, C Luccarini, PDP Pharoah}

Strangeways Laboratory for Genetic Epidemiology, Department of Oncology and Department of Public Health, University of Cambridge, UK Breast Cancer Research 2005, 7(Suppl 2):S.40 (DOI 10.1186/bcr1083)

Background Fifteen per cent to $20 \%$ of the familial clustering of breast cancer is explained by the effects of highly penetrant mutations in BRCA1 and BRCA2. Modelling based on the patterns of familial aggregation of breast cancer in the relatives of cases ascertained on a population basis suggests that much of the remaining familial effect is due to the combined effects of genetic variants individually of small effect. The numbers of such variants, their allele frequencies and the strength of their effects is not known.

Methods We have carried out association studies to search for common variants (minor allele frequency $>5-10 \%$ ) that contribute to predisposition,. To date we have studied 400 SNPs in 110 genes using a two-stage study design, in which a first set of 2300 cases and controls is analysed and all SNPs with a significance value of $P<0.1$ or better are then tested in a second, similar, case/control set.

Results No individual SNP has, to date, given a $P$ value for association (based on genotype distribution) lower than $10^{-4}$. A number of SNPs give $P$ values between $10^{-2}$ and $10^{-4}$, depending on the genetic model that is chosen for the analysis. Most of these are probably false positives, the consequence of multiple testing. However, comparison of the distribution of $P$ values across the entire study set with that expected if there were no genetic effect suggests that some of these are probably true positive associations, representing low-level predisposing effects.
Conclusions A candidate gene approach is slow and relatively expensive, and has not so far yielded unequivocal positive results for any individual gene. The 'genetic architecture' of breast cancer - that is, the number and characteristics of predisposing genetic variants - is still not known. In an attempt to elucidate this and to hasten the process of gene discovery, we have initiated (with collaborators in the UK and at Perlegen Science Inc.) a genome-wide scan. Again we use a two-stage approach. In the first stage we will evaluate 266,000 SNPs in 400 breast cancer cases and 400 controls. The cases will be 'enriched' for genetic effects by choosing those with a family history, tested negative for BRCA1/2 mutation. In the second stage, $\sim 5 \%$ of SNPs will be further evaluated in 4600 cases and controls. A final stage of evaluation for positives from the second stage, and from our earlier studies, will require analysis of a further, very large $(\sim 10,000)$, case/control set, which we hope to assemble through international collaboration.

\section{S.41 \\ Functional genomic approaches to breast cancer $\mathbf{R}$ Bernards}

Division of Molecular Carcinogenesis, The Netherlands Cancer Institute, Amsterdam, The Netherlands

Breast Cancer Research 2005, 7(Suppl 2):S.41 (DOI 10.1186/bcr1084)

Background One of the major remaining deficits in our understanding of the human genome is that information regarding gene function is available for only one-quarter of the approximately 30,000 genes. Many of these hitherto anonymous genes are potential targets for the development of new anti-cancer drugs. It is therefore important to functionally annotate the tens of thousands of genes for which this information is currently lacking. My laboratory has developed functional genetic approaches to obtain information regarding gene function using high-throughput screens in mammalian cells. We have developed both gain-of-function genetic screens (using retroviral cDNA expression libraries) and loss-of-function genetic screens (using vectorbased RNA interference libraries) to carry out large-scale genetic screens in mammalian cells. We focus on the central growth-regulatory pathways that are most frequently deregulated in cancer.

Methods We have designed a mammalian expression vector (pSUPER), which directs the synthesis of short hairpin transcripts (shRNAs) that are processed intracellularly into siRNA-like molecules. This vector mediates persistent inhibition of gene expression in a highly specific fashion. We have used this vector to stably suppress expression of individual members of several cancer-relevant gene families.

Results We used a retroviral derivative of the PSUPER siRNA vector to generate a large collection of siRNA vectors that each target a single gene for suppression. In total, we constructed a set of 23,742 siRNA vectors that together target 7914 human genes for suppression by RNA interference. Furthermore, we developed a very efficient way to identify biologically active shRNA vectors in a large population of vectors, a technology that we named 'siRNA bar code screening'. We will present two applications of this technology to study major questions in breast cancer. First, we have used the RNAi library to identify genes whose suppression causes resistance to anti-hormonal therapy (tamoxifen resistance). In addition, we have used RNAi technology to ask how clinical resistance to the Her2/neu/ErbB2targeted therapeutic Herceptin can arise.

Conclusion RNA interference is a powerful technology to identify genes that are causally involved in disease processes. Application of this technology to breast cancer may greatly expedite the development of novel diagnostics and therapeutics for the treatment of this disease.

\section{References}

1. Brummelkamp TR, Nijman SMB, Dirac AMG, Bernards R: Loss of the cylindromatosis tumour suppressor inhibits apoptosis by activating NF-kB. Nature 2003, 424:797-801.

2. Brummelkamp TR, Bernards R: New tools for functional mammalian cancer genetics. Nat Rev Cancer 2003, 3:781-789.

3. Berns K, Hijmans EM, Mullenders J, Brummelkamp TR, Velds A, Kerkhoven RMH, Madiredjo M, Nijkamp W, Weigelt B, Agami R, et al:: A large-scale RNAi screen in human cells identifies new components of the p53 pathway. Nature 2004, 428:431-437. 


\subsection{2}

Proteomic approaches to early detection of breast cancer

JE Celis 1,2, P Gromovi,2, JMA Moreira',2, T Cabezón1,2, E Friis'1,3, F Rank ${ }^{1,4}$, I Gromova ${ }^{1,2}$

${ }^{1}$ The Danish Centre for Translational Breast Cancer Research (DCTB), Copenhagen, Denmark; ${ }^{2}$ Department of Proteomics in Cancer, Institute of Cancer Biology, Danish Cancer Society, Denmark; ${ }^{3}$ Department of Breast and Endocrine Surgery, Rigshospitalet, Denmark; ${ }^{4}$ Department of Pathology, The Centre of Diagnostic Investigations, Rigshospitalet, Denmark

Breast Cancer Research 2005, 7(Suppl 2):S.42 (DOI 10.1186/bcr1085)

The completion of the human genome as well as the explosion of novel technologies within genomics, proteomics and functional genomics promise to have a major impact on clinical practice, as these technologies are expected to accelerate the translation of basic discoveries to the clinical practice. In particular, proteomic technologies are expected to play a key role in the study and treatment of cancer as they provide invaluable resources to define and characterize regulatory and functional networks, to investigate the precise molecular defect in diseased tissues and biological fluids, and to develop specific reagents to precisely pinpoint a particular disease or stage of a disease. For drug discovery, proteomics assist with powerful tools for identifying new clinically relevant drug targets, and provide functional insight for drug development.

Today, the application of novel technologies from proteomics and functional genomics to the study of cancer is rapidly shifting to the analysis of clinically relevant samples such as fresh biopsy specimens and fluids, as their use will accelerate the translation of basic discoveries. Being a patient-oriented organisation, The Danish Cancer Society catalysed in 2002 the creation of a multidisciplinary research environment, the DCTB, to fight breast cancer. The DCTB hosts scientists working in various areas of preclinical cancer research (cell cycle control, invasion and microenvironmental alterations, apoptosis, cell signalling, and immunology) with clinicians (surgeons, oncologists) and pathologists in an integrated, mission-oriented, discovery-driven translational research environment. The unifying concept behind our experimental strategy is the use of multiple experimental paradigms for the prospective analysis of clinically relevant samples obtained from the same patient, along with the systematic integration of the biological and clinical data.

Here I will describe our efforts to apply proteomics approaches to search for markers for early detection of breast cancer using the newly characterized interstitial fluids recovered from fresh tissue biopsies of both normal (NIF) and tumour (TIF) origin. The protein composition of the fluids is strikingly different to that of serum and cyst fluids, although they share some of their major components. The TIF is highly enriched in proteins that are either secreted via the classic endoplasmic reticulum/Golgi pathway, shed by membrane vesicles (membrane blebbing), or externalized by plasma membrane transporter. Hundreds of primary translation products, as well as post-translational modifications, have so far been identified using a combination of procedures that include mass spectrometry, two-dimensional gel immunoblotting, and cytokine and signalling pathway-specific antibody arrays. The workflow to biomarker discovery as well as recent developments will be discussed.

\subsection{3}

\section{Dissection of molecular pathways of cancer by high- throughput biochip technologies and RNA interference O Kallioniemi}

Medical Biotechnology, VTT Technical Research Centre of Finland; University of Turku, Finland

Breast Cancer Research 2005, 7(Suppl 2):S.43 (DOI 10.1186/bcr1086)

Objective Our aim is to identify new molecular targets and mechanisms for therapeutic intervention in cancer. To achieve this aim, we develop and apply multiple high-throughput technologies including 'in silico' screening as well as technologies for molecular, cellular and clinical discovery research. Finally, data integration from these technology platforms is applied to facilitate interpretation and prioritization of the findings.

In silico screening In order to make use of the exponential increase of published data on gene expression arrays, we have launched a project to acquire and make use of these data as a discovery resource. We currently have data on 5700 samples analyzed on the Affymetrix gene expression platform stored in our relational database. These samples include, for example, 64 normal tissues/cell types, 43 tumor types, many other diseases as well as functional experiments; altogether 84 million data points. We have developed methods to mine these data to identify tissue-specific and disease-specific expression patterns of transcripts, to identify gene coexpression profiles, to explore networks of gene regulation as well as methods to interpret new microarray experiments. In silico transcriptomic screening makes it possible to generate dozens of testable hypotheses for laboratory analysis based on datasets that are much larger and more extensive than any single academic laboratory can afford to generate. Analysis of gene expression profiles across hundreds of tissue and tumor types, diseases and experimental manipulations generates novel, often unexpected, insights of gene function as well as of the underlying biology and medicine.

Molecular screening Large cohorts of clinical samples are now being investigated not only at the RNA level by gene expression profiling, but also at the DNA-level using comparative genomic hybridization (CGH) arrays for analysis of somatic genetic alterations or SNP arrays for studies of allelic gains and losses. There is also an emerging interest for large-scale proteomic and metabolic profiling. It will be increasingly important to integrate multiple levels of molecular profiling data to gain new insights and comprehensive views on mechanisms of cancer development. We are applying single-gene resolution oligo-CGH arrays and integrating these data with gene expression information on the same samples. The increased $\mathrm{CGH}$ resolution has highlighted several microdeletions as well as small amplifications, whose impact on gene expression can be substantial and highly specific. This has led to an opportunity for rapid identification of genes that may be targets of genetic alterations in cancer. As demonstrated by several recently approved drugs for cancer, such mutated genes represent attractive targets for the development of effective cancer-specific therapeutics.

Functional screening using RNA interference The molecular profiling of DNA expression patterns, RNA expression patterns or protein expression patterns in patient samples is not sufficient for implicating these molecules or molecular mechanisms as therapeutic targets. It is also necessary to generate functional information on such genes and pathways. Towards this aim, we have developed a high-throughput screening system that is composed of a robotic, automated platform for the analysis of up to 20,000 functional experiments with living cells at a time using the 384-well microplate format. Cells are dispensed into culture wells, exposed to siRNAs or small molecule compounds, incubated for 1-3 days, washed, and stained with phenotype-specific markers for cell growth, cell cycle distribution or induction of apoptosis. The results are read by plate readers or cell cytometers. Functional studies with RNAi libraries (e.g. 1000-10,000 siRNAs) have implicated genes whose targeting by RNAi is lethal to specific cancer types, such as breast cancer. Integration of such functional RNAi data with gene expression and aCGH data has enabled us to identify genes that are targets of genetic alterations and whose expression is required for the maintenance of the malignant phenotype. Such genes represent attractive candidate drug targets.

Clinical screening Data on molecular targets arising from functional in vitro studies need to be corroborated in studies of large-scale clinical sample cohorts in order to verify that such molecular targets are relevant in clinical patient samples. A number of technologies are being developed towards this aim. First, the in silico screening transcriptomics database with 5700 samples has made it possible to develop an approach for 'in silico clinical validation'. It is possible to determine the expression levels of any gene across a very large number of tumor types and normal sample types. Second, more established 
technologies, such as tissue microarrays, facilitate the analysis of individual DNA, RNA and protein targets in thousands of arrayed patient samples, typically from formalin-fixed tumors. Tissue microarray analysis with antibody binders produces definitive clinical data on the expression of therapeutic protein targets, and enables quantitation of drug target distributions at the population level (target epidemiology). In order to further increase the throughput of molecular analyses, we are developing methods to print tissue lysates from frozen samples in an array format. Tissue protein lysate arrays will help to automate and expand antibody-based validation of molecular targets in large cohorts of tissue specimens.

Conclusion In summary, high-throughput technologies can be applied in the 'in silico', molecular, cellular and clinical screening studies, thereby substantially increasing the information and insights that can be obtained on a single platform. Integration of data from the various highthroughput platforms will facilitate a deeper, mechanistic interpretation and understanding of cancer biology, which is an essential requirement for the development of next-generation targeted therapies.

\subsection{4 \\ Envisioning new targets and new approaches for molecular-based cancer therapeutics \\ JA Tainer}

Lawrence Berkeley National Laboratory, Skaggs Institute for Chemical Biology and The Scripps Research Institute, Department of Molecular Biology, La Jolla, California, USA

Breast Cancer Research 2005, 7(Suppl 2):S.44 (DOI 10.1186/bcr1087)

We aim to understand how genome maintenance and stress responses are coordinated by dynamically changing, multiprotein complexes. Reversible complexes involve composite interfaces from modular preformed and unstructured regions that provide strong, specific contacts from the combination of relatively weak, modular interactions. These individual modular interaction sites, which allow protein exchanges and pathway progression, also provide possible targets for new therapeutic strategies. DNA genetic integrity and cancer avoidance depends upon the structure-specific repair and replication nuclease flap endonuclease (FEN-1) and upon the trimeric processivity factor PCNA. FEN-1 and PCNA complex structures and mutational results provide a coherent model for DNA substrate recognition and PCNA activation of FEN-1. Together, these structural and mutational results support an interface exchange hypothesis for coordinated transfer of DNA intermediates during PCNA-mediated processes. We have furthermore defined analogous interface exchange as an important coordinating factor for homologous recombination repair (HRR) of DNA double-strand breaks. The Mre11/Rad50 (MR) complex that first recognizes DNA double-strand breaks and Rad51 complexes that promote recombination are essential for DNA break repair and recombination processes. To help understand the molecular mechanism of the MR complex in DSB repair, we determined crystal structures of Mre11 and Rad50 catalytic domains (Mre11cd and pfRad50cd), their interface, DNA interactions, and a unique $\mathrm{Zn}$-hook linking the $600 \AA$ coiled-coil domain of Rad50. The MR complex must handoff the DNA ends to Rad51, which catalyzes homologous pairing and exchange between dsDNA and ssDNA via orchestrated interactions with BRCA2, Rad52, and other HRR proteins. We determined an atomic structure of a polymeric fulllength RAD51 homolog to reveal atomic details of quaternary assembly, we experimentally test a proposed BRC repeat-induced RAD51 disassembly mechanism and we address the molecular mechanism for the orchestrated interactions of RAD51 in HRR. The Rad51 structure reveals a polymerization motif involving an interdomain linker key for quaternary assembly. Structural and mutational results suggest how differences in RAD51 ring and helical nucleoprotein filament assemblies may regulate ATPase activity. A RAD51 filament assembly based on 3D EM reconstructions and crystallographic interfaces suggests a novel role for RAD51 N-terminal domains in binding dsDNA within a large outer groove. By taking advantage of the simpler organization of archaeal recombination systems, our structural establish at the molecular level how BRC repeats disrupt RAD51 assembly and direct RAD51 to form foci in cells in response to DNA damage. Our results help support a molecular mechanism for the ordered interactions of HRR partners BRCA2, RAD52, RAD54 and RAD55 by protein-mediated and DNA-mediated exchanges of RAD51 polymer interface elements. To achieve accurate structural information on these difficult but biologically relevant molecular complexes, we have designed and developed the Structurally Integrated Biology for Life Sciences beamline at the Advanced Light Source. This provides a unique resource for $\mathrm{X}$-ray diffraction characterizations of both static and time-resolved solution states of macromolecular machines.

\section{Poster abstracts}

\section{P1.01}

\section{Cigarette smoking and breast cancer risk among non-drinking women}

IT Gram 1, T Braaten', HO Adami'2, E Lund', E Weiderpass'2

1 Institute of Community Medicine, University of Tromso, Norway; 2Department of Medical Epidemiology and Biostatistics, Karolinska Institute, Stockholm, Sweden

Breast Cancer Research 2005, 7(Suppl 2):P1.01 (DOI 10.1186/bcr1088)

Background The relationship between smoking and the risk of breast cancer has been unclear for several decades, and whether or not young women should be warned about smoking as a possible cause of breast cancer remains controversial [1-3]. A recent pooled analysis of 53 epidemiologic studies found that the positive relationship with smoking and breast cancer was present only among those consuming alcohol, an established cause of breast cancer [4]. The purpose of this report was to examine the effect of smoking on breast cancer risk among non-drinking women in a large population-based cohort of women, many of whom started smoking as teenagers.

Methods We followed 102,098 women, aged 30-50 years, completing a mailed questionnaire at recruitment to the NorwegianSwedish Cohort Study in 1991/1992, through December 2000. Altogether, 1240 women were diagnosed with incident, invasive breast cancer. We used Cox proportional hazard regression models to estimate the relative risk of breast cancer associated with different measures of smoking initiation, duration, and intensity adjusting for confounding variables. We conducted analyses on the non-drinking study population, and especially among non-drinking women who had smoked for at least 20 years.

Results We identified 214 breast cancer cases among the 19,288 non-drinking women. Compared with never smokers, women who smoked for at least 20 years, and initiated smoking prior to their first birth (relative risk $=1.75 ; 95 \%$ confidence interval $=1.02-2.99$ ), before menarche (relative risk $=1.66 ; 95 \%$ confidence interval $=$ $0.79-3.49$ ) or before age 15 (relative risk $=2.20 ; 95 \%$ confidence interval $=0.92-5.30$ ), had an increased risk. In contrast, women who had smoked for at least 20 years, but started after their first birth, did not experience an increased breast cancer risk (relative risk $=0.97$; $95 \%$ confidence interval $=0.44-2.12$ ).

Conclusion Our results support the notion that non-drinking women who start smoking as teenagers and who continue to smoke for at least 20 years may increase their risk of breast cancer.

\section{References}

1. Chaturvedi $P$ : Does smoking increase the risk of breast cancer? Lancet Oncol 2003, 4:657-658.

2. Beral V, Doll R, Peto R, Reeves G: Does smoking increase the risk of breast cancer? Lancet Oncol 2003, 4:658.

3. IARC Working Group: Tobacco smoke and involuntary smoking (IARC Monographs on the Evaluation of Carcinogenic Risks to Humans). IARC Monogr 2004, 83:11452.

4. Hamajima N, Hirose K, Tajima K, Rohan T, Calle EE, Heath CW, $\mathrm{Jr}$, et al:: Alcohol, tobacco and breast cancer - collaborative reanalysis of individual data from $\mathbf{5 3}$ epidemiological studies, including 58,515 women with breast cancer and 95,067 women without the disease. Br J Cancer 2002, 87:1234-1245. 


\section{P1.02}

Mutation screening of BRCA1, BRCA2 and CHEK2*1100delC in Slovak HBOC families

S Cierniková1, M Tomka1, M Kovac', v Stevurkova', v Bella², J Novotny ${ }^{3}$, V Zajac ${ }^{1}$

${ }^{1}$ Laboratory of Cancer Genetics, Cancer Research Institute of Slovak Academy of Sciences, Bratislava, Slovakia; ${ }^{2}$ National Cancer Institute, Bratislava, Slovakia; ${ }^{3}$ Clinic of Oncology, VFN and 1 st LF UK, Prague, Czech Republic

Breast Cancer Research 2005, 7(Suppl 2):P1.02 (DOI 10.1186/bcr1089) Background Germline mutations in BRCA1 and BRCA2 genes account for most of the hereditary breast and ovarian cancers (HBOC). Recently, other low-penetrance candidate genes involved in breast cancer susceptibility, CHEK2 and ATM, have emerged. We have initiated mutation screening of suspected $\mathrm{HBOC}$ families to improve health care for affected individuals and their asymptomatic relatives in the Slovak Republic.

Methods We performed a mutational analysis of the entire coding region of the $B R C A 1$ gene in 110 suspected $\mathrm{HBOC}$ families from all parts of Slovakia. DNA from peripheral blood lymphocytes was analyzed by the combination of a single-strand conformation polymorphism (SSCP), heteroduplex analysis (HDA), protein truncation test and direct DNA sequencing. Initial screening of the first 16 BRCA2 exons in $40 \mathrm{HBOC}$ families was performed using SSCP and HDA. To investigate the presence of the CHEK2*1100delC variant, DNA from 65 selected patients was screened by denaturing high-performance liquid chromatography.

Results To date, we have detected eight previously described mutations (185delAG, C39R, C61G, 962del4, L1013X, 2072del4, 3819del5, 5382insC) and one novel BRCA1 deletion (2057delCAGTGAAGAG) in DNA samples of $16 \mathrm{HBOC}$ families. Initial screening for germline mutations in BRCA2 revealed one recurrent mutation, 6696delTC, in the very large $\mathrm{HBOC}$ kindred. Out of 67 asymptomatic relatives from families with the identified BRCA1 or BRCA2 mutations, 31 were found to be mutation carriers. These individuals are included in the Special Program of Preventive Health Care. Mutation analysis has also identified 17 different polymorphisms or unclassified sequence variants scattered through the BRCA1 and BRCA2 genes in 48 patients from the analyzed collection of $\mathrm{HBOC}$ families. From these, the most frequent alterations were L771L, E1038G and K1183R, presented in 11,10 and nine patients, respectively. We have not detected any 1100 delC variant of the CHEK2 gene in 65 tested HBOC patients. Conclusion In order to identify patients and families predisposed to hereditary breast and ovarian cancer, we searched for germline mutations in two major breast and ovarian cancer susceptibility genes, $B R C A 1$ and BRCA2. Screening of the whole coding sequence of the $B R C A 1$ gene and the first 16 exons of $B R C A 2$ in selected families has identified the spectrum of mutations previously reported in HBOC families of different ethnic or geographic origin. In addition, we found the novel BRCA1 mutation 2057del10, detected in a very high-risk family characterized by different types of cancer (breast and ovarian cancer, male breast cancer, lung cancer, colorectal cancer and testicular cancer). The first results from a small collection of 65 patients did not confirm the correlation between the CHEK2*1100delC variant and breast cancer in these cases, according to the fact that none of the tested DNA was found to be positive. Although the screening of BRCA2 gene needs to be completed, the achieved results represent the first molecular characterization of Slovak HBOC families.

Acknowledgements This work was supported by the Slovak Government Grant Agency VEGA (No. 2/3089) and by Project No. 2003SP 5102808 00/028 0801 from the National program 'Use of Cancer Genomics to Improve the Human Population Health'.

\section{P1.03}

Identification of novel sequence alterations and the functional analysis of the BRCA1 promoter/5'-UTR in families from Upper Silesia, Poland

J Pamuła, M Krześniak, H Zientek, W Pękala, M Rusin, E Grzybowska

Department of Tumor Biology, Maria Skłodowska-Curie Memorial Cancer Center and Institute of Oncology, Gliwice, Poland

Breast Cancer Research 2005, 7(Suppl 2):P1.03 (DOI 10.1186/bcr1090) Background The human $B R C A 1$ gene is under transcriptional control of two alternative promoters, $\alpha$ and $\beta$, that drive the transcription of exon $1 \mathrm{a}$ or exon $1 \mathrm{~b}$, respectively. Additionally, the $5^{\prime}$-UTR region, encompassing both exons 1, contains multiple putative and functional regulatory sequences. At the RNA level each exon 1 is linked, by splicing, with exon 2 that contains the translation start site. The aim of this study was to search for sequence alterations within the BRCA1 promoter and 5'-UTR in patients with breast and/or ovarian cancer, and to assess whether these sequence variants influence activity of the $B R C A 1$ promoter region.

Methods The $5^{\prime}$ region of the BRCA1 gene, containing promoters $\alpha$ and $\beta$ as well as exons $1 \mathrm{a}$ and $1 \mathrm{~b}$ and the fragment of intron 1 , was sequenced in 87 breast/ovarian cancer cases. All patients had a strong family history of breast and ovarian cancer, but were found mutationnegative in our previous search for founder mutations in BRCA1 (185delAG, 300T/G, 4153delA, 5382insC) and BRCA2 (6174delT, $9631 \mathrm{de}(\mathrm{C})$. The frequency of the 2223delAAAAA deletion was assessed using allele-specific PCR amplification (ASA) in a larger group of breast/ovarian cancer patients fulfilling the aforementioned criteria. The functional significance of sequence variants within the $5^{\prime}$ UTR of $B R C A 1$ was analyzed by luciferase assay. A $1.5 \mathrm{~kb}$ DNA region encompassing minimal BRCA1 promoter and $5^{\prime}$-UTR, both wild type and variant sequence, was cloned into the pGL3 vector containing the luciferase reporter gene. The luciferase activity reflected the influence of the sequence alterations on the transcriptional activity of the BRCA1 promoter and other gene regulatory regions.

Results We found several sequence variants within the examined noncoding region of $B R C A 1$. The frequency of the largest sequence alteration found, deletion 2223delAAAAA (according to the Acc. U37574) within exon 1b, was determined in a group of 150 patients. Three families have been identified bearing the said deletion. We also found two linked nucleotide substitutions (2642A $>T, 2743 T>C$ ) in $B R C A 1$ intron 1. The functional impact of the most frequent sequence alterations was examined in lung cancer cell line $\mathrm{NCl}-\mathrm{H} 1299$ and breast cancer cell line MCF7. In the MCF7 cell line all tested variants of BRCA1 promoter $/ 5^{\prime}$-UTR showed lower activity than the control wildtype sequence, while in $\mathrm{NCl}-\mathrm{H} 1299$ cells the variant promoter/5'-UTR activity was higher than the control. However, observed differences of luciferase activity were not statistically significant.

Conclusion Our luciferase assay showed that sequence variants detected in our study within the BRCA1 promoter/5'-UTR do not change the functional activity of the BRCA1 promoter in the experimental system that we have used, and may not be associated with an increased risk of breast and ovarian cancer. The detailed results of the analyses will be presented.

\section{P1.04}

High prevalence of a BRCA1 gene founder mutation, 5083del19, in unselected breast-ovarian cancer patients from Southern Italy: genotype-phenotype correlations F Baudi', AM Lavecchia ${ }^{2}$, B Quaresima', MC Faniello', L De Paola', W D'Amico', F Fabiani', G Cuda', FS Costanzo'1, S Venuta ${ }^{1}$

'Dipartimento di Medicina Sperimentale e Clinica 'G Salvatore', Facoltà di Medicina e Chirurgia, Università degli Studi Magna Græcia di Catanzaro, Italy; ${ }^{2}$ Unità Operativa di Anatomia Patologica, 'Azienda Ospedaliera Pugliese-Ciaccio', Catanzaro, Italy

Breast Cancer Research 2005, 7(Suppl 2):P1.04 (DOI 10.1186/bcr1091) Background The implementation of informative BRCA1 testing programs is aided by acquisition of population-specific genetic data. 
From this it emerges that a number of mutations have been found repeatedly, and specific mutations are common in defined populations. This can be referred to a founder effect that in human genetics refers to the presence of genetic disorders that are either endemic to an isolated population or are very rare elsewhere. In a previous populationbased study we described the first example of a founder BRCA1 mutation in Italy, with a strong recurrence of the 5083del19 in high-risk patients all of Calabrian origin selected for family history of the disease [1]. The same mutation accounts in Canada for a significant percentage of women of Italian ancestry with breast-ovarian cancer [2].

The aim of this study was to investigate the extent to which the 5083 del19 mutation contributed to breast-ovarian cancer incidence in Calabria, and to perform some genotype-phenotype correlations.

Methods We tested 70 paraffin-embedded tissue specimens from a consecutive series of breast or ovarian cancer cases. It is estimated that our tissue archive collects more than $90 \%$ of incident cancers in the Catanzaro area. Archival tissue samples were genotyped only for the 5083del19 founder mutation. Immunohistochemical staining of several markers (i.e. ER, PR, Ki67, p53, HER2, CK5/6) as well as some pathological features (i.e. histology, grade) were evaluated.

Results Seven out of the 70 samples screened for this BRCA mutation had mutations. Haplotype analysis revealed a common ancestor. All cases aged in premenopausal years. The phenotype of the 5083del19 BRCA1-associated breast tumours appears characterized by a lack of expression of hormone receptors and is generally associated with high proliferation markers and poorly differentiated aspects. All cases were ductal invasive carcinomas and showed a 'basal-like' portrait according to expression profiling studies [3]. The median age at diagnosis of the ovarian cancer carriers was 49 years and the histological type was serous adenocarcinoma.

Conclusion A predominant BRCA1 gene founder mutation associated with a high risk of early onset breast cancer and ovarian cancer and unfavourable immunophenotype features has been identified and found to occur in a restricted geographical area, thereby allowing timely and cost-effective mutation screening using blood samples or archival histological material.

Acknowledgements This work was supported by grants from COFIN 2003 (MURST), CLUSTER C-04 (MURST), Progetto Speciale Regione Marche (Ministero Salute) and AIRC.

References

1. Baudi F, Quaresima B, Grandinetti C, Cuda G, Faniello C, Tassone $P$, Barbieri V, Bisegna R, Ricevuto E, Conforti S, et al.: Evidence of a founder mutation of BRCA1 in a highly homogeneous population from southern Italy with breast/ovarian cancer. Hum Mutat 2001, 18:163-164.

2. Nedelcu R, Liede A, Aube J, Finch A, Kwan E, Jack E, Narod SA, Randall S, Hugel L, Clark K: BRCA mutations in Italian breast/ovarian cancer families. Eur J Hum Genet 2002, 10:150-152.

3. Foulkes WD, Stefansson IM, Chappuis PO, Begin LR, Goffin JR, Wong N, Trudel M, Akslen LA: Germline BRCA1 mutations and a basal epithelial phenotype in breast cancer. J Natl Cancer Inst 2003, 95:1482-1485.

\section{P1.05}

\section{Screening for germline rearrangements in BRCA1} and BRCA2 in Norwegian families with breast or breast/ovarian cancer

M Van Ghelue', M Ingebrigtsen ${ }^{1}$, HMF Riise Stensland', L Mæhle' ${ }^{2}$, J Apold ${ }^{3}$, P Møller², V Marton', C Jonsrud1

${ }^{1}$ Department of Medical Genetics, University Hospital North Norway, Tromso, Norway; ${ }^{2}$ Section for Genetic Counselling, Cancer Genetics, The Norwegian Radium Hospital, Oslo, Norway; ${ }^{3}$ Centre for Medical Genetics and Molecular Medicine, Haukeland University Hospital,

Bergen, Norway

Breast Cancer Research 2005, 7(Suppl 2):P1.05 (DOI 10.1186/bcr1092) Standard PCR-based mutation detection strategies performed on the mostly aimed at identifying changes in the coding sequences and in the donor-acceptor splice sites. Hence, mutations in the promoter and the untranslated regions, and large rearrangements, are not detected by these methods. To assess the importance of BRCA1 and BRCA2 alterations that are neglected by standard screening methods, we monitored germline rearrangements in these genes using 'multiplex ligation-dependent probe amplification' technology [1]. One hundred and seventy-nine Norwegian breast and ovarian cancer families were screened for rearrangements in BRCA1 while 97 families were tested for aberrations in BRCA2. Whereas no rearrangements were detected in BRCA2, four distinct deletions were found in BRCA1. Those deletions originating by Alu-mediated homologous recombination include: exons 1-13, exons $3-16$, exons $8-13$ and exon 23 , respectively. The large $23.8 \mathrm{~kb}$ deletion excluding exons $8-13$ in $B R C A 1$ has been found both in the French and British breast cancer population [2-4]. The deletions of exons 1-13, exons 3-16 and exon 23 have not been previously reported.

References

1. Schouten JP, McElgunn CJ, Waaijer R, Zwijnenburg D, Diepvens $F$, Pals G: Relative quantification of 40 nucleic acid sequences by multiplex ligation-dependent probe amplification. Nucleic Acids Res 2002, 30:e57.

2. Puget N, Stoppa-Lyonnet D, Sinilnikova OM, Pages S, Lynch HT, Lenoir GM, Mazoyer S: Screening for germ-line rearrangements and regulatory mutations in BRCA1 led to the identification of four new deletions. Cancer Res 1999, 59:455-461.

3. Gad S, Caux-Moncoutier V, Pages-Berhouet S, Gauthier-Villars M, Coupier I, Pujol P, Frenay M, Gilbert B, Maugard C, Bignon YJ, et al.: Significant contribution of large BRCA1 gene rearrangements in 120 French breast and ovarian cancer families. Oncogene 2002, 21:6841-6847.

4. Bunyan DJ, Eccles DM, Sillibourne J, Wilkins E, Thomas NS, Shea-Simonds J, Duncan PJ, Curtis CE, Robinson DO, Harvey JF, Cross NC: Dosage analysis of cancer predisposition genes by multiplex ligation-dependent probe amplification. $\mathrm{Br} J$ Cancer 2004, 91:1155-1159.

\section{P1.06}

Hereditary breast cancer - a spectrum of pathogenic mutations and unknown variants of BRCA1 and BRCA2 genes in the Czech Republic: efficiency of testing and clinical follow-up

L Foretova, M Lukesova, P Vasickova, M Navratilova, H Pavlu, J Kuklova, V Urbankova, D Hanouskova, B Dvorackova, E Machackova

Department of Cancer Epidemiology and Genetics, Masaryk Memorial Cancer Institute, Brno, Czech Republic

Breast Cancer Research 2005, 7(Suppl 2):P1.06 (DOI 10.1186/bcr1093)

Background Germline mutations in the highly penetrant cancer susceptibility genes $B R C A 1$ and $B R C A 2$ cause genetic predisposition to breast and ovarian cancers. Molecular genetic testing of pathogenic mutations in these two genes is an effective method for breast cancer risk prediction. Genetic counselling and testing has been provided to high-risk women in our institute since 1997. Until now 589 probands (580 women and nine men) with breast/ovarian cancer have been tested for BRCA1/2 germline mutations.

Methods Genetic counselling was performed by a medical geneticist in our institute or in other genetic centres of the Czech Republic. Informed consent was signed in all tested individuals. For genetic testing the non-radioactive protein truncation test of exon 11 of BRCA1 and exons 10 and 11 of BRCA2 were used, followed by heteroduplex analysis of the remaining exons with their splice sites and by sequencing. The frequency of unknown variants was tested in a control group of healthy women older than 60 years without a positive family history of breast/ovarian cancer. 
Results The pathogenic mutation was found in 179 of 589 tested probands (30\%), 106 in the BRCA1 gene and 73 in the BRCA2 gene. The frequency of detected mutations was calculated in different categories of family history and in sporadic cases. Mutation was found in 86 of 213 (overall 40.4\%, hereditary ovarian cancer [HOC] + hereditary breast and ovarian cancers [HBOC] 63.6\%, hereditary breast cancer $[\mathrm{HBC}] 30.8 \%)$ families with three or more cases of breast or ovarian cancer (bilateral cancer was counted as two cases), in 66 of 258 (overall 25.6\%, HOC + HBOC 46.8\%, HBC 22.1\%) families with two breast or ovarian cases, in four of 14 (28.6\%) probands with sporadic bilateral breast cancer first diagnosed before age 50 , in three of seven (42.9\%) women with sporadic bilateral ovarian cancer under age 50 , in 11 of 12 (91.7\%) women with sporadic breast and ovarian cancer diagnosed at any age, in six of 66 $(9.1 \%)$ women with sporadic unilateral breast cancer before age 40 , in zero of 10 women with sporadic unilateral ovarian cancer before age 40 , in three of six $(50 \%)$ males with familial form of breast cancer, and in one of three (33.3\%) males with sporadic breast cancer.

There are five most frequent mutations in the Czech population, which represent $56.8 \%$ of all mutations found (c.5385dupC, c.3819_3823delGTAAA and c.300T>G in BRCA1; c.8138_8142delCCTTT and c.8765_8766delAG in BRCA2). Overall 14 novel pathogenic mutations were detected, eight of them have been published by our group $[1,2]$ and the other six mutations have not yet been published. A spectrum of 28 variants (13 in BRCA1 and 15 in $B R C A 2)$ with unknown clinical significance was found. The most frequent variant was c.5075G $>$ A, p.M1652l, in the BRCA1 gene (in 22 cases), but the frequency of this variant in a control population was $6.3 \%$. The missense mutation in the BRCA1 gene c.172T>A, p.M18K, was detected three times in HBOC and HBC families, and is very probably pathogenic (localised in a highly conserved structure of the Ring finger domain); it was not detected in a control group. Another two variants were detected in a control population with frequency 1.6\%: IVS24/c.9485-16T>C and c.10323delCins11 in the BRCA2 gene.

By predictive testing, positive carrier status was disclosed in 163 individuals of 395 tested. The clinical follow-up was offered to all individuals at high risk. The high-risk clinic at $\mathrm{MMCl}$ is following 62 healthy carriers, 139 healthy people at risk, 47 patients-carriers and 29 high-risk patients with negative testing result. From these, 14 patients and three healthy carriers underwent prophylactic mastectomy, 26 patients and four healthy carriers underwent prophylactic adnexectomy. According to the questionnaire for diagnoses of depression CES-D instituted before testing, 51\% of women did not report any signs of depression, $27 \%$ reported very mild, $14 \%$ middle and $8 \%$ severe signs of depression. A psychologist in our institute follows 27 women. No severe psychological complications of testing have so far been reported.

Conclusion Overall, pathogenic mutation was disclosed in 30\% of all our tested individuals with familial or sporadic breast (or ovarian) cancer. At least one ovarian cancer in a high-risk family increases twice the likelihood of mutation detection. Fourteen novel mutations and a spectrum of unknown variants were detected. Prophylactic mastectomy is not so frequently accepted by mutation carriers as in Western Europe, but there is an increasing tendency especially in young women. The quality of preventive care in other institutions and the psychosocial influence of genetic testing are now being investigated.

Acknowledgement Supported by the Internal Grant Agency of the Ministry of Health of the Czech Republic (NR-8022-3 and NR-8213-3). References

1. Machackova E, Damborsky J, Valik D, Foretova L: Novel germline BRCA1 and BRCA2 mutations in breast/ovarian cancer families from the Czech Republic. Hum Mutat 2001, 18:545.

2. Foretova L, Machackova E, Navratilova, M Pavlu H, Hruba M, Lukesova M, Valik D: BRCA1 and BRCA2 mutations in women with familial or early-onset breast/ovarian cancer in the Czech Republic. Hum Mutat 2004, 23:397-398.

\section{P1.07}

Genetic determinants of breast cancer characteristics and outcome in women under $\mathbf{5 0}$ years of age MK Schmidt', A van der Plas', SR de Kemp', S Klaver'1, B Nota', B Maertzdorf', R de Groot ${ }^{1}$, R Udo', VTHBM Smit'2, A Broeks', JL Peterse', FE van Leeuwen', RAEM Tollenaar'2, LJ van 't Veer'

${ }^{1}$ Department of Pathology, Department of Epidemiology and Department of Surgery, The Netherlands Cancer Institute, Amsterdam, The Netherlands; ' ${ }^{2}$ Department of Surgery, Department of Pathology and Department of Clinical Genetics, Leiden University Medical Center, Leiden, The Netherlands

Breast Cancer Research 2005, 7(Suppl 2):P1.07 (DOI 10.1186/bcr1094) Germline mutations in BRCA1 and BRCA2 account for approximately $2-3 \%$ of breast cancers while CHEK2*1100de/C may account for an additional $0.7 \%$ [1]. To date, several small studies have suggested a worse outcome of survival in BRCA1 and BRCA2 carriers. However, the evidence is inconsistent and most studies were subject to different types of bias. An increased risk for contralateral breast cancer, especially in interaction with radiotherapy, for CHEK2*1100de/C carriers has been shown [2]. Only one study has so far evaluated the impact of CHEK2 ${ }^{*} 1100$ de/C on survival, showing a worse disease-free survival compared with control breast cancer patients [3].

Our aim is to evaluate breast cancer survival and to determine risk estimations for the development of contralateral breast or ovarian cancer (as a second primary), as well as to evaluate tumour characteristics, in BRCA1/2 and CHEK2*1100de/C carriers in an unselected, non-family based, retrospective cohort of breast cancer patients diagnosed under age 50 . The cohort to be evaluated will include approximately 5000 patients, treated in several Dutch hospitals between 1973 and 1995. Tissue blocks from these patients are being obtained and, after coding, about 70 BRCA1/2 founder and recurrent mutations, representing approximately $72 \%$ of the Dutch BRCA1/2 mutations, and the CHEK 1100 de/C mutation are being determined.

Data for 1700 patients from the Netherlands Cancer Institute and the Leiden University Medical Center are being completed. We have so far found, in 1255 samples, 4.1\% BRCA1/2 carriers (41 BRCA1 and 11 BRCA2 mutations) and $3.8 \%$ CHEK2*1100de/C, with no overlap among these groups. An interim analysis showed that $B R C A 1$ tumours seem to have less favourable prognostic characteristics while BRCA carriers have an OR of 3 for contralateral breast cancer compared with the non-BRCA carriers. Genetic determinants of tumour characteristics, risk for contralateral breast cancer and survival of CHEK2*1100de/C carriers will be presented.

References

1. The CHEK2 Breast Cancer Case-Control Consortium: CHEK2*1100deIC and susceptibility to breast cancer: a collaborative analysis involving 10,860 breast cancer cases and 9,065 controls from 10 studies. Am J Hum Genet 2004, 74: $1175-1182$

2. Broeks A, de Witte L, Nooijen A, Huseinovic A, Klijn JG, van Leeuwen FE, Russell NS, Van 't Veer LJ: Excess risk for contralateral breast cancer in CHEK2*1100delC germline mutation carriers. Breast Cancer Res Treat 2004, 83:91-93.

3. de Bock GH, Schutte M, Krol-Warmerdam EM, Seynaeve C, Blom J, Brekelmans CT, Meijers-Heijboer H, van Asperen CJ, Cornelisse $\mathrm{CJ}$, Devilee $\mathrm{P}$, et al: Tumour characteristics and prognosis of breast cancer patients carrying the germline CHEK2*1100delC variant. J Med Genet 2004, 41:731-735. 


\section{P1.08}

Low-dose ionizing radiation significantly increases the risk of breast cancer among BRCA1/2 mutation carriers in the International BRCA1/2 Carrier Cohort Study (IBCCS)

FE Van Leeuwen ${ }^{1}, \mathbf{N}$ Andrieu ${ }^{2}$, DF Easton ${ }^{3}$, J Chang-Claude ${ }^{4}$, R Brohet ${ }^{1}$, E Cardis 5 , AC Antoniou ${ }^{3}$, S Peock ${ }^{3}$, C Nogues ${ }^{6}$, MA Rookus ${ }^{1}$, DE Goldgar ${ }^{5}$, on behalf of EMBRACE, GENEPSO, GEO-HEBON and the IBCCS collaborators' group

${ }^{1}$ The Netherlands Cancer Institute, Department of Epidemiology, Amsterdam, The Netherlands; ${ }^{2}$ INSERM EmiOO-06 et Service de Biostatistique de l'Institut Curie, France; ${ }^{3}$ Cancer Research UK, Genetic Epidemiology Unit, Department of Public Health and Primary Care, University of Cambridge, UK; ${ }^{4}$ Division of Epidemiology, German Cancer Research Center, Heidelberg, Germany; ${ }^{5}$ International Agency for Research on Cancer, Lyon, France; ${ }^{6}$ Centre René Huguenin, Saint Cloud, France

Breast Cancer Research 2005, 7(Suppl 2):P1.08 (DOI 10.1186/bcr1095) Background Women who carry germline mutations in the BRCA1 and BRCA2 genes are at a greatly increased risk of breast cancer (BC). Numerous studies have shown that exposure to ionizing radiation is a risk factor for BC. Because of the role of the BRCA proteins in DNA repair it is plausible that women who carry mutations in these genes might be more sensitive to ionizing radiation than women in the general population. We therefore determined the role of low-dose ionizing radiation in carriers of a BRCA1 or BRCA2 mutation.

Methods A retrospective cohort study of 1601 female BRCA1/2 carriers, of whom 879 were affected with $\mathrm{BC}$ at the time of interview, was performed. Exposure data were analyzed using a weighted Cox proportional hazards model. We assessed the relative risk of $\mathrm{BC}$ as a function of exposure to chest $\mathrm{X}$-rays as assessed by questionnaire.

Results In the entire cohort, any reported exposure to chest X-rays was associated with a significantly increased risk of $B C$ (hazard ratio $[\mathrm{HR}]=$ $1.54,95 \%$ confidence interval $[\mathrm{Cl}]=1.1-2.1, P=0.007)$ compared with those reporting never having had an $\mathrm{X}$-ray. This risk was increased in carrier women aged 40 and younger $(\mathrm{HR}=1.97,95 \% \mathrm{Cl}=1.3-2.9$, $P<0.001)$, and in women born after $1949(\mathrm{HR}=3.6,95 \% \mathrm{Cl}=$ 2.1-6.2, $P<0.001$ ). Estimated risks were highest in women exposed to $\mathrm{X}$-rays before age 20 only, particularly those born in later birth cohorts $(\mathrm{HR}=4.85,95 \% \mathrm{Cl}=2.2-10.9, P<0.001)$.

Conclusions The observed patterns of risk are consistent with those found in previous studies of radiation and $\mathrm{BC}$, but the extent of the risk increase in BRCA carriers appears to exceed several-fold that observed for other radiation-exposed cohorts. The results of this study have important implications for the use of $\mathrm{X}$-ray imaging in BRCA $1 / 2$ carriers, particularly before age 20 .

\section{P1.09}

\section{Clinical outcome for BRCA1 and BRCA2 mutation} carriers after contralateral prophylactic mastectomy MK Schmidt' 1 , TC van Sprundel' ${ }^{2}$, MA Rookus ${ }^{1}$, R Brohet $^{1}$, CJ van Asperen ${ }^{2}$, EJTh Rutgers', RAEM Tollenaar ${ }^{2}$, U van 't Veer'

${ }^{1}$ Department of Pathology, Department of Epidemiology and Department of Surgery, The Netherlands Cancer Institute, Amsterdam, The Netherlands; ' ${ }^{2}$ Department of Surgery and Department of Clinical Genetics, Leiden University Medical Center, Leiden, The Netherlands Breast Cancer Research 2005, 7(Suppl 2):P1.09 (DOI 10.1186/bcr1096) Studies have shown that (bilateral) prophylactic mastectomy in genetically predisposed populations reduces the risk of breast cancer. Since $B R C A 1$ and $B R C A 2$ germline mutation testing became widely available (1995), breast cancer patients with a family history of breast cancer have also been tested. After having been identified as a $B R C A 1$ or BRCA2 carrier, some women decide to undergo a intensive surveillance. The impact of this choice on contralateral breast cancer incidence and survival is still unknown.

We identified a cohort of 148 female BRCA1 or BRCA2 mutation carriers (115 and 33 , respectively) who previously were treated for unilateral invasive breast cancer stage I-Illa. Seventy-nine women underwent a CPM, while the other women remained under intensive surveillance. The mean follow-up was 3.5 years and started at the time of CPM or at the date of mutation testing, whichever came last (i.e. on average, 5 years after diagnosis of the first breast cancer).

One woman developed an invasive contralateral primary breast cancer after CPM, whereas six were observed in the surveillance group $(P<0.001)$. CPM reduced the risk of contralateral breast cancer by $91 \%$, independent of the effect of bilateral prophylactic oophorectomy (BPO). At 5-year follow-up, overall survival was 94\% for the CPM group versus $77 \%$ for the surveillance group $(P=0.03)$. Unexpectedly, this difference in survival was mostly due to higher mortality related with the first breast cancer and ovarian cancer in the surveillance group. After adjustment for BPO in a multivariate Cox analysis, the CPM effect on overall survival was no longer significant.

Our data show that CPM markedly reduces the risk of contralateral breast cancer among BRCA1 or BRCA2 mutation carriers with a history of breast cancer. Longer follow-up is needed to study the impact of CPM on contralateral breast cancer specific survival. The choice for CPM is highly correlated with that for BPO while only BPO so far leads to a significant improvement in overall survival.

\section{P1.10}

Oral contraceptives and breast cancer risk in the International BRCA1/2 Carrier Cohort Study (IBCCS)

MA Rookus', RM Brohet ${ }^{1}$, N Andrieu ${ }^{2}$, AC Antoniou ${ }^{3}$, J Chang-Claude ${ }^{4}$, DF Easton ${ }^{3}$, S Peock ${ }^{3}$, C Noguès ${ }^{2}$, FE van Leeuwen ${ }^{1}$, DE Goldgar ${ }^{5}$, on behalf of the IBCCS Collaborating Group

${ }^{1}$ The Netherlands Cancer Institute, Department of Epidemiology, Amsterdam, The Netherlands; 2 I'Institut Curie, Paris, France; ${ }^{3}$ Cancer Research UK, University of Cambridge, UK; ${ }^{4}$ German Cancer Research Center, Heidelberg, Germany; ${ }^{5}$ International Agency for Research on Cancer, Lyon, France

Breast Cancer Research 2005, 7(Suppl 2):P1.10 (DOI 10.1186/bcr1097) Background The marked reduction of the risk of breast cancer following a prophylactic oophorectomy illustrates that endogenous hormones play an important role in the etiology of breast cancer among BRCA1/2 mutation carriers, as they do in the general population. In the general population the use of oral contraceptives has been associated with a slightly increased risk. Little is so far known about the safety of oral contraceptives among BRCA1/2 carriers, who have much higher premenopausal background rates of breast cancer.

Methods A retrospective cohort study was performed using an international cohort of 1601 BRCA1/2 mutation carriers. A timedependent proportional hazard Cox regression was used, stratified for birth cohort, gene, country of residence and relatedness. All analyses were adjusted for prophylactic oophorectomy and number of full-term pregnancies. To reduce possible testing bias, the analyses were weighted to achieve the rate of breast cancer within the cohort as a priori estimated for BRCA1/2 mutation carriers.

Results We found a slightly increased risk of breast cancer for BRCA/12 mutation carriers who ever used oral contraceptives, with an adjusted hazard ratio of 1.47 (95\% confidence interval $=1.16-1.87$ ). The risk increase did not vary according to various aspects of oral contraceptive use, such as time since stopping, duration of use, age at start, and calendar year at start. In addition, the risk increase was similar for BRCA1 and BRCA2 mutation carriers.

Conclusion Use of oral contraceptives seems to be associated with a slightly increased relative risk of breast cancer among BRCA1/2 mutation carriers, comparable with the general population. However, due to the high background rates of breast cancer among BRCA1/2 carriers, oral contraceptive use may result in a considerable absolute excess risk of breast cancer, if the association is causal. 
Acknowledgements This work was supported by NIH Award CA81203, Cancer Research UK, the INHERIT BRCAs research program, the Fondation de France and the Ligue Nationale Contre le Cancer, and the Dutch Cancer Society.

\section{P1.11}

\section{Genome-wide scanning for linkage in 56 Dutch breast cancer families selected for a minimal probability of being due to BRCA1 or BRCA2}

RA Oldenburg1,2, K Kroeze-Jansema', JJ Houwing ${ }^{3}$, J Kraan', JG Klijn ${ }^{4}$, N Hoogerbrugge ${ }^{5}$, MJ Ligtenberg ${ }^{5}$, CJ van Asperen ${ }^{1}$, HF Vasen ${ }^{6}$, H Meijers-Heijboer ${ }^{2}$, CJ Cornelisse ${ }^{7}$, P Devilee $^{1,7}$

${ }^{1}$ Department of Human \& Clinical Genetics, Leiden University Medical Center, Leiden, The Netherlands; ${ }^{2}$ Department of Clinical Genetics, Erasmus University, Rotterdam, The Netherlands; ${ }^{3}$ Department of Medical Statistics, Leiden University Medical Center, Leiden, The Netherlands; ${ }^{4}$ Daniel den Hoed Cancer Centre, Rotterdam, The Netherlands; ${ }^{5}$ Department of Human Genetics, University of Nijmegen, The Netherlands; ${ }^{6}$ Foundation for the Detection of Hereditary Tumors, Leiden, The Netherlands; ${ }^{7}$ Department of Pathology, Leiden University Medical Center, Leiden, The Netherlands

Breast Cancer Research 2005, 7(Suppl 2):P1.11 (DOI 10.1186/bcr1098) Background A conventional model of inherited breast cancer susceptibility is that disease risks are affected by mutations in a small number of genes causing a high risk of the disease and by a larger number of lower risk gene variants probably interacting together [1]. Model-based linkage analysis in multiple-case families, followed by positional cloning, led to the identification of BRCA1 and BRCA2. The cancer risks conferred by mutations in these genes are now well established, but together they explain only approximately $25 \%$ of the overall excess familial risk. Families with at least four cases of breast cancer and at least one case of ovarian cancer can be attributed largely to BRCA1. Multiple-case families with at least one case of male breast cancer are mainly due to BRCA2. But the majority of families with four or five cases of female breast cancer diagnosed before the age of 60 are not due to BRCA1 or BRCA2. This has been taken as evidence that one or more moderate-risk to high-risk breast cancer susceptibility genes still remain to be identified [2].

Methods There have been several linkage claims since BRCA1 and BRCA2 were identified, but none of these have been replicated in other, often larger, studies. Most studies, however, are heavily underpowered to detect a new breast cancer locus by linkage in the presence of substantial genetic heterogeneity. The Breast Cancer Linkage Consortium is currently compiling genome-wide linkage data on approximately 200 families in which the role of BRCA1 or BRCA2 has been excluded with $>90 \%$ certainty. This comprises the largest post-BRCA1/2 linkage search effort in breast cancer families in the world to date. These families are characterised by the presence of at least three cases of breast cancer diagnosed before the age of 60 , and no cases of ovarian or male breast cancer.

Results Here we present the Dutch contribution to this effort, including GENEHUNTER analysis of 56 families in which 208 patients were genotyped at 410 microsatellite markers. Allowing for heterogeneity, there were two regions, on $1 q$ and $15 q$, that gave lod scores of $1.40(\alpha=0.12)$ and $1.19(\alpha=0.29)$, respectively. Lod scores for all other markers were $<1.0$. In non-parametric analysis, lod scores $>1.0$ were found for one or more markers at $4 p, 6 q, 7 q, 9 p, 15 q$, and $21 q$. All of these regions are currently being followed up by additional flanking marker typings.

References

1. Antoniou AC, Pharoah PDP, McMullan G, Day NE, Stratton MR, Peto J, Ponder BJ, Easton DF: A comprehensive model for familial breast cancer incorporating BRCA1, BRCA2 and other genes. Br J Cancer 2002, 86:76-83.

2. Ford D, Easton DF, Stratton M, Narod S, Goldgar D, Devilee P, Bishop DT, Weber B, Lenoir G, Chang-Claude J, et al., Breast Cancer Linkage Consortium: Genetic heterogeneity and penetrance analysis of the BRCA1 and BRCA2 genes in breast cancer families. Am J Hum Genet 1998, 62:676-689.

\section{P1.12}

\section{Who gets cancer?}

BA Nexø ${ }^{1}$, U Vogel2 ${ }^{2}$ K Overvad ${ }^{3}$, O Raaschou-Nielsen4, A Tjønneland ${ }^{4}$

1 Institute of Human Genetics, University of Aarhus, Denmark;

2 National Institute of Occupational Health, Copenhagen, Denmark;

${ }^{3}$ Department of Clinical Epidemiology, Aalborg Hospital and Aarhus University Hospital, and Department of Epidemiology and Social Medicine, University of Aarhus, Denmark; ${ }^{4}$ Institute of Cancer Epidemiology, The Danish Cancer Society, Copenhagen, Denmark

Breast Cancer Research 2005, 7(Suppl 2):P1.12 (DOI 10.1186/bcr1099) We have looked for genetic differences influencing cancer risk in the general human population. One human chromosomal region that recent data indicate as important for cancer risk is 19q13.2-3, more specifically a $69 \mathrm{~kb}$ region including the genes $X P D, R A I, A S E 1$ and $E R C C 1$. We and others have produced evidence of association between this region and occurrence of a variety of cancers, including basal cell carcinoma, melanoma, lung cancer, glioma, breast cancer, bladder cancer, and possibly head and neck cancer (see for instance [1]). A recent search along the region for markers with maximal association to basal cell carcinoma has led to a focus on the gene RAl [2]. Moreover, the effect appears to be strongest among fairly young persons ( $<56$ years of age).

These studies mainly involved analysis of single nucleotide polymorphisms in unrelated cases and controls. However, other studies have mapped a glioma tumor suppressor function to an almost overlapping region using deletions in tumor DNA [3]. Finally, a recent genome-wide scan for chromosomal regions associated with aggressive prostate cancer located the strongest effector in a $6 \mathrm{Mb}$ region, which includes the four genes mentioned [4].

It appears that this region of chromosome 19 , most probably the gene $R A l$, often contains a genetic variant, which increases the risk of several cancers among fairly young humans. $R A /$ produces an inhibitor of NF-KB, and may thus be involved in apoptosis, which makes it very easy to rationalize its importance for cancer. In addition, studies of lung cancer have produced evidence for an independent effector within the $69 \mathrm{~kb}$ region of interest, possibly related to DNA repair.

References

1. Nexø BA, Vogel U, Olsen A, Ketelsen T, Bukowy Z, Thomsen BL, Wallin $H$, Overvad K, Tjønneland A: A specific haplotype of single nucleotide polymorphisms on chromosome 19q13.2-3 encompassing the genes RAI is indicative of postmenopausal breast cancer before age 55. Carcinogenesis 2003, 24:899-904.

2. Rockenbauer E, Bendixen MH, Bukowy Z, Yin J, Jacobsen NR, Hedayati MA, Vogel U, Grossman L, Bolund L, Nexø BA: Association of chromosome 19q13.2-3 with basal cell carcinoma: tentative delineation of an involved region using data for single nucleotide polymorphisms in two cohorts. Carcinogenesis 2002, 23:1149-1153.

3. Hartmann C, Johnk L, Kitange G, Wu Y, Ashworth LK, Jenkins RB Louis DN: Transcript map of the 23.7 Mb D19S112-D19S246 candidate tumor suppressor region on the long arm of chromosome 19. Cancer Res 2002, 62:4100-4108.

4. Slager SL, Schaid DJ, Cunningham JM, McDonnell SK, Marks AF, Peterson BJ, Hebbring SJ, Anderson S, French AJ, Thibodeau SN: Confirmation of linkage of prostate cancer aggressiveness with chromosome 19q. Am J Hum Genet 2003, 72:759-762.

P1.13

DNA polymorphisms of several genes and predisposition to breast cancer

D Zaletayev', V Artamonov', D Mikhaylenko', L Lyubchenko², M Nemtsova ${ }^{1}$

1 Institute of Molecular Medicine at Moscow Medical Academy, Moscow, Russian Federation; ${ }^{2}$ Blokhin Cancer Research Center RAMS, Moscow, Russian Federation

Breast Cancer Research 2005, 7(Suppl 2):P1.13 (DOI 10.1186/bcr1100)

Background Breast cancer is one of the major cancers around the world but its etiology is still not well understood. Only about $50 \%$ of 
the disease is associated with known risk factors including highpenetrance genes and lifestyle factors. Candidate low-penetrance genes are involved in a variety of pathways; for example, DNA damage by free radicals. The enzymes involved in this mechanism are $\mathrm{N}$-acetyltransferase2 (NAT2) and manganese superoxide dismutase (MnSOD). NAT2 catalyzes acetylation of aromatic amines and hydrasines and forms of free radicals, and MnSOD catalyzes their dismutation. Genes TGFBR1 and RGS19IP1 encode proteins that take part in transforming growth factor beta signaling, mainly resulting in inhibition of cell proliferation. The first exon of TGFBR1 contains a polymorphic GCG repeat; (GCG) ${ }_{6}$ was previously reported as the tumor susceptibility allele associated with some types of cancer. The $5^{\prime}$-untranslated region of RGS19/P1contains a CGG repeat, the polymorphism of which had not been studied before.

Methods SNPs of NAT2 and MnSOD were detected by the PCRRFLP method, alleles of TGFBR1 were detected by electrophoresis and RGS19IP1 alleles were detected by fragment analysis on 123 breast cancer cases and 121 controls.

Results We have assessed the frequency of frequent allelic variants of NAT2 (NAT2*4 [wild type], NAT2*5 [T341C], NAT2*6 [G590A], NAT2*7 [G857A]). The NAT2*11 allele in the Russian sample was found as well. Our breast cancer cases had statistically significant positive association with $N A T 2{ }^{*} 6 /{ }^{*} 6$ or $N A T 2 * 11$ (33.4\% vs $11.0 \%$; $P=0,0005 ; \mathrm{OR}=3.06$ [95\% $\mathrm{Cl}=1.62-5.77]$, cases vs controls). The frequency of the NAT2*5 and NAT2*7 alleles was not significantly elevated in our breast cancer sample compared with controls. The MnSOD gene was studied for polymorphism of valine $(\mathrm{V})$ versus alanine (A) in the leader peptide at position 16. The V/V genotype $\left(\mathrm{MroNI}^{-/-}\right)$was associated with decreased risk of breast cancer (24.4\% vs $38.0 \% ; P=0.0268$; OR $=0.53[95 \% \mathrm{Cl}=0.03-0.91]$ ). The risk of breast cancer decreased in a combination of NAT2*4, NAT2*5 or NAT2*7 alleles and V/V genotype MnSOD $(20.3 \%$ vs $36.1 \% ; P=0.0068$; OR $=0.45$ [95\% Cl 0.25-0.79]).

Our breast cancer cases had statistically significant positive association with the (GCG) ${ }_{6}$ allele of TGFBR1 (13.8\% vs $6.1 \%$, $P=0.016, \mathrm{OR}=2.47[95 \% \mathrm{Cl}=1.21-5.07])$. Statistically significant positive association with the genotype $6 A / 9 A(20.2 \%$ vs $11.6 \%$, $\alpha=0.05$, OR $=1.92[95 \% \mathrm{Cl}=1.05-3.49]$ ) was also revealed. Genotype $6 A / 9 A$ can be used as a predisposition marker, the relative risk for this genotype carrier being increased 1.7-fold. We have identified a polymorphism of the CGG repeat in RGS19IP1 with at least eight alleles: four major $\left(\mathrm{CGG}_{10}-\mathrm{CGG}_{13}\right)$, with frequencies $20.4 \%, 41.6 \%, 27.6 \%$ and $10.4 \%$, respectively; the rest of the alleles are rare with overall frequency of $1 \%$ in control; heterozygosity, 0.729 . Our breast cancer cases had statistically significant positive association with the rare alleles $(4.7 \%$ vs $1 \%, P=0.01, \mathrm{OR}=4.92$ $[95 \%, \mathrm{Cl}=1.42-17.1])$.

Conclusion All polymorphisms tested could be used as a panel of markers for detecting families and individuals of high breast cancer risk

\section{P1.14}

\section{Large-scale single nucleotide polymorphism analysis of candidates for low-penetrance breast cancer genes \\ A Vega ${ }^{1,2}$, A Salas ${ }^{2,3}$, C Phillips², B Sobrino², B Carracedo', C Ruíz-Ponte ${ }^{1,2}$, R Rodríguez-López ${ }^{4}$, G Rivas $^{4}, J^{\prime}$ Benítez ${ }^{4}$, A Carracedo $1,2,3$}

${ }^{1}$ Fundación Pública Galega de Medicina Xenómica, Hospital Clínico Universitario, Universidad de Santiago de Compostela, Galicia, Spain; ${ }^{2}$ Centro Nacional de Xenotipado, Hospital Clínico Universitario, Santiago de Compostela, Galicia, Spain; ${ }^{3}$ Unidade de Xenética, Instituto de Medicina Legal, Facultad de Medicina, Universidad de Santiago de Compostela, Galicia, Spain; ${ }^{4}$ Departamento de Genética Humana, Centro Nacional Investigaciones Oncológicas, Madrid, Spain Breast Cancer Research 2005, 7(Suppl 2):P1.14 (DOI 10.1186/bcr1101) $B R C A 1$ and $B R C A 2$ are high-penetrance genes that account for around $25 \%$ of families with hereditary breast cancer [1]. Given that no additional high-penetrance susceptibility genes have been found to be involved in breast cancer, it has been proposed that different genetic backgrounds due to the combination of low-penetrance genes (polygenic mechanism) could explain the remaining familial breast cancer risk [2]. Hence there is much interest in the search for lowpenetrance gene/variants for breast cancer, which exist with high prevalence in the general population.

Single nucleotide polymorphisms (SNPs) have emerged as genetic markers of choice because of their high density and relatively even distribution in the human genomes [3,4], and are being using for fine mapping of disease loci and for candidate gene association studies. Approximately 10 million SNPs have been identified across the human genome and new technologies are available today for high-throughput genotyping.

In this study we used the SNPlex ${ }^{\mathrm{TM}}$ (Applied Biosystems, Foster City, CA, USA) high-throughput genotyping platform, which allows the study of up to 48 SNPs simultaneously, to study 984 SNPs of 92 cancerrelated genes, in a total of 480 female cases of breast cancer and 480 female controls.

Gene selection was made on the basis of their involvement in different cancer pathways and genes: DNA reparation, cell cycle control, BRCA1-associated binding proteins, and so on. SNP selection was performed using an indirect approach (1 SNP/10 kb) and based on the individual allele frequency (FAM $\leq 10 \%$ ) in the European population, using public and private SNP databases and bioinformatics tools (dbSNP, HapMap, Sequenom Real SNP, PUPASNPI Ensembl, and Celera, among others).

To date, 415 SNPs from 44 genes have been genotyped in nine SNPlex pools. A case-control analysis was conducted for the 318 remaining SNPs. Preliminary results showed association in 24 SNPs from 12 candidate genes $(P<0.05)$. We will present the analysis of the remaining 48 genes at the time of the congress.

Acknowledgments This work was supported by grants from the Ministerio de Sanidad y Consumo (Fondo de Investigación Sanitaria; Instituto de Salud Carlos III, PI030893; SCO/3425/2002) and Genoma España (CeGen; Centro Nacional de Genotipado; Nodo Santiago de Compostela).

References

1. Ford D, Easton DF, Stratton M, Narod S, Goldgar D, Devilee P, Bishop DT, Weber B, Lenoir G, Chang-Claude J, et al.: Genetic heterogeneity and penetrance analysis of the BRCA1 and BRCA2 genes in breast cancer families. Breast Cancer Linkage Consortium. Am J Hum Genet 1998, 62:676-689.

2. Pharoah PD, Antoniou A, Bobrow M, Zimmern RL, Easton DF, Ponder BA: Polygenic susceptibility to breast cancer and implications for prevention. Nat Genet 2002, 31:33-36.

3. Kruglyak L: Prospects for whole-genome linkage disequilibrium mapping of common disease genes. Nat Genet 1999, 22: 139-144.

4. Venter JC, Adams MD, Myers EW, Li PW, Mural RJ, Sutton GG, et al:: The sequence of the human genome. Science 2001, 291:1304-1351.

\section{P1.15}

\section{Chromosome-wide pharmacogenetics: localisation and linkage disequilibrium of genes coding for ROS metabolism and signalling}

H Edvardsen', B Kulle', GI Grenaker-Alnæs', AC Syvänen³, AL Børresen-Dale ${ }^{1}$, A Frigessi², VN Kristensen ${ }^{1}$

${ }^{1}$ Department of Genetics, The Norwegian Radium Hospital, Oslo, Norway; ${ }^{2}$ Section of Medical Statistics, University of Oslo, Norway; ${ }^{3}$ Department of Medical Sciences, Uppsala University, Uppsala, Sweden

Breast Cancer Research 2005, 7(Suppl 2):P1.15 (DOI 10.1186/bcr1102) Background Pharmacogenetic studies provide data of increasingly many SNPs in relation to response to various treatments of psychological disorders, cardiovascular disease and cancer. Simultaneously, the HapMap and projects like it reveal the linkage disequilibrium (LD) map of unselected genes in different human 
populations. To what extent does this knowledge of the LD domains affect previous findings from pharmacogenetic studies of single candidate SNPs? Here we select candidate genes as part of a given functional pathway, and report their chromosomal localization and extent of LD.

Methods A total of 193 breast cancer patients have been genotyped for 725 SNPs in 206 genes selected through the candidate gene approach. Two hundred and fifty-three of the SNPs have also been genotyped in a cohort of 109 healthy Norwegian women. SNPs that had a discovery rate lower than $75 \%$ were excluded. Hardy-Weinberg equilibrium was calculated prior to further statistical analysis of LD. LD estimations were made using PHASE, a program that implements methods for calculating haplotypes from population genotype data [1]. Results The 725 SNPs were divided between 206 different genes with 1-20 SNPs per gene distributed on all chromosomes. Initially, SNPs were grouped in clusters containing a minimum of three SNPs with no more than $100 \mathrm{~kb}$ between neighbouring SNPs. Based on the PHASE output, $D^{\prime}$ and the $P$ value of the Fisher's exact test were calculated. We observed strong LD in 74 genes, and 10 genes were split into more than one LD domain. Furthermore, neighbouring clusters of genes were studied for common LD. Genotype frequencies and the extent of LD were compared in a case-control study when possible.

Conclusion Our findings are restricted by our choice of genes and the number of SNPs per gene. Nevertheless, they reveal LD between SNPs in multiple genes, which have been previously studied in separate and independent studies. This notion of the existing LD may be of potential value in designing new pharmacogenetic studies.

Reference

1. Stephens M, Donnelly P: A comparison of Bayesian methods for haplotype reconstruction from population genotype data. Am J Hum Genet 2003, 73:1162-1169.

\section{P1.16}

\section{Comparison of methods for pharmacogenomics:} SNaPshot, SNPstream UHT, Nanogen, and RFLP GIG Alnæs', FE Johansen', SH Nordgard', B Hihn'2, H Edvardsen', VI Hilden ${ }^{1}$, PE Lønning ${ }^{3}$, A-C Syvänen ${ }^{4}$, A-L Børresen-Dale', VN Kristensen'1

'Department of Genetics, The Norwegian Radium Hospital, Oslo, Norway; ${ }^{2}$ Nanogen Europe BV, Helmond, The Netherlands;

${ }^{3}$ Department of Medicine, Section of Oncology, Haukeland University Hospital, Bergen, Norway; ${ }^{4}$ Uppsala University, Department of Medical Sciences, Uppsala, Sweden

Breast Cancer Research 2005, 7(Suppl 2):P1.16 (DOI 10.1186/bcr1103) The inter-individual variation of response to cancer chemotherapy and radiation therapy has a substantial genetic component and is a subject of pharmacogenetic studies. Our goal is to develop assays to analyze selected single nucleotide polymorphisms (SNPs) in genes with proven relevance for pharmacogenetics. Today's research in the field needs technological platforms for which the demands both in terms of quality and throughput are high. Pharmacogenomics utilizes various genotyping techniques as well as gene-expression studies in studying the effect of different pharmaceuticals as well as the pathways that influence them. Here we provide an overview of the platforms currently in use, and discuss their efficiency, precision and technological stability. The amount of hands-on time needed and the costeffectiveness are discussed. We also compared the success rate of several methods. SNaPshot is a homogeneous-phase-based primer extension method and SNPstream UHT is a solid-phase-based primer extension method. The Nanogen chip employs binding of a biotinylated probe to a chip coated with hydrogel enriched with streptavidin. This universal design presents the possibility to perform both genotyping as well as gene-expression experiments, even on the same chip. Results were compared with the classical enzymatic restriction enzyme fragment polymorphism (RFLP) assay. The initial set included eight SNPs in the X-ray cross-complementing group 1 protein, XRCC1; APEX nuclease (multifunctional DNA repair enzyme) 1, APEX1; multidrug resistance gene, MDR1; gluthation-S-transferase $\mathrm{P} 1$,
GSTP1; and tumor protein p53, TP53. Functional SNPs in these genes have been shown to influence the therapeutic effect of a series of anti-cancer drugs such as 5-fluorouracyl, doxorubicin, etoposide, thioguanine and irinotekan. Three series of 36 breast cancer patients treated with 5-fluorouracyl and mitomycin, of 92 breast cancer patients treated with doxorubicin and of 109 control individuals have been genotyped. This resulted in 1527 genotypes. Of these, 391 had been analysed by SNaPshot, 900 by SNPstream UHT, 454 by Nanogen and 966 by RFLP. The success rate was $91 \%$ for SNaPshot, $97 \%$ for SNPstream UHT, 96\% for Nanogen, and 99\% for RFLP. When comparing the methods pairwise, the lowest variation was observed between SNPstream UHT and Nanogen (0.4\%). SNPstream UHT and Nanogen had similar rates of discrepancy when compared with RFLP: $3.2 \%$ and $2.6 \%$, respectively. Close re-analysis of those samples revealed false calls due to incomplete cutting during the RFLP. The highest observed dis-concordance observed was between $\mathrm{SNaPshot}$ and SNPstream UHT (8.9\%). Careful evaluation of genotyping quality is needed both for large epidemiological studies and smaller clinical trials, and especially in prospective pharmacogenetic studies of separate individuals in clinical settings.

\section{P1.17}

\section{Genetic polymorphisms in the $5^{\prime}$ flanking region of glutathione S-transferase P1 affect promoter methylation}

JA Rønneberg1', J Tost'2, GI Grenaker-Alnæs'1, T Sørlie', T Kristensen 3 , I Gut'2 , A-L Børresen-Dale', VN Kristensen'1

${ }^{1}$ Department of Genetics, Institute for Cancer Research, The Norwegian Radium Hospital, Oslo, Norway; ${ }^{2}$ Centre National de Genotypage, Evry, France; ${ }^{3}$ Department of Molecular Biosciences, University of Oslo, Norway

Breast Cancer Research 2005, 7(Suppl 2):P1.17 (DOI 10.1186/bcr1104) Glutathione-S-transferase P1 (GSTP1) is involved in thiol-mediated detoxification and breakdown of reactive oxygen species created by anticancer drug exposure. GSTP1 is also an inhibitor of c-Jun Nterminal kinase 1, a kinase involved in stress response, apoptosis and cellular proliferation. Hypermethylation of the GSTP1 promoter has been associated with gene silencing in prostate cancer, kidney cancer, and breast cancer, among others. Although frequently described, the mechanism underlying promoter hypermethylation of the GSTP1 gene is poorly understood. It has been reported that an ATAAA repeat of the GSTP1 promoter separates methylated from unmethylated CpGs in normal prostate tissue [1]. These separate methylation domains are lost in prostate cancer, and methylation extends throughout the whole promoter region. It has been proposed that hypermethylation of GSTP1 requires a combination of gene silencing and random seeds of methylation in prostate cancer cells, and that these combinatorial effects lead to histone deacetylation and subsequent chromatin remodeling [2]. To further elucidate the mechanisms underlying the hypermethylation of the GSTP1 promoter, we genotyped the (ATAAA) repeat and the linked SNPs in positions $-354,-288,-287$ and -282 in the GSTP1 promoter and we performed methylation analysis using mass spectrometry in tumor DNA from 82 breast cancer patients. The role of the different allelic variants on methylation status of the GSTP1 promoter and expression levels was assessed. We quantitatively determined the methylation status of six CpGs spanning the transcription start site of the GSTP1 promoter: $-22,+8,+14,+38$, +47 and +55 . The average percentage methylation for each individual CpG for the 82 tumor samples analyzed was $16.9 \%, 30.3 \%, 18.2 \%$, $21.2 \%, 18.6 \%$ and $8.1 \%$, respectively. The average percentage methylation for all CpGs in all tumor samples was $19 \%$. There was a correlation between the degree of methylation of the individual $\mathrm{CpGs}$ and their neighboring CpGs $(P<0.001)$. When correlating the extent of methylation to the mRNA levels previously assessed by whole genome gene-expression profiling of the same tumors, a significant inverse correlation was observed $(P<0.01)$. The methylation status of the three CpGs closest to the transcriptional start site was more highly associated with the level of GSTP1 mRNA expression than the CpGs 
further downstream of the +1 site. Furthermore, we observed differences in the degree of GSTP1 promoter methylation between the different tumor subclasses defined by whole-genome microarray analysis [3]. The methylation of the GSTP1 promoter was significantly lower in the basal subtype compared with the luminal subtype, which corresponded to elevated GSTP1 mRNA levels in the basal subtypes [4]. We further analyzed the impact of the most frequent haplotype structure of the GSTP1 promoter in relation to the extent of methylation, and a correlation was observed $(P=0.003)$ suggesting that haplotype structures can affect de novo methylation of adjacent sequences.

References

1. Millar DS, et al.: J Biol Chem 2000, 275:24893-24899.

2. Stirzaker C, et al.: Cancer Res 2004, 64:3871-3877.

3. Perou CM, et al:: Nature 2000, 406:747-752.

4. Troester MA, et al.: Cancer Res 2004, 64:4218-4226.

\section{P1.18}

The rare ERBB2 variant lle654Val is associated with an increased familial breast cancer risk

B Frank'1, K Hemminki1,2, M Wirtenberger'1, JL Bermejo', P Bugert ${ }^{3}$, R Klaes ${ }^{4}$, RK Schmutzler ${ }^{5}$, B Wappenschmidt ${ }^{5}$, CR Bartram ${ }^{4}$, B Burwinkel ${ }^{1}$

${ }^{1}$ Division of Molecular Genetic Epidemiology, German Cancer Research Center, Heidelberg, Germany; ${ }^{2}$ Department of Biosciences at Novum, Karolinska Institute, Huddinge, Sweden; ${ }^{3}$ nstitute of Transfusion Medicine and Immunology, Red Cross Blood Service of Baden-Württemberg-Hessia, University of Heidelberg, Faculty of Clinical Medicine, Mannheim, Germany; ${ }^{4}$ Institute of Human Genetics, University of Heidelberg, Germany; ${ }^{5}$ Division of Molecular GynaecoOncology, Department of Gynaecology and Obstetrics, Clinical Center University of Cologne, Germany

Breast Cancer Research 2005, 7(Suppl 2):P1.18 (DOI 10.1186/bcr1105)

Background Overexpression of the proto-oncogene ERBB2 (HER2/NEU) has been observed in $20-30 \%$ of breast cancers involving ligand-independent activation and more aggressive growth behaviour, reduced response to chemotherapy and hormonal therapy, as well as poor prognosis. Genetic alterations within ERBB2 have been shown to induce carcinogenesis and metastasis.

Methods The ERBB2 variants Ile654Val, Ile655Val and Ala1170Pro were investigated for their influence on familial breast cancer risk by sequencing and TaqMan allelic discrimination.

Results The case-control study analysing 348 German familial breast cancer cases and 960 controls showed no significant association of Ile655Val and Ala1170Pro with familial breast cancer risk. Differences in haplotype frequencies between cases and controls could not be detected either. The ERBB2 variant lle654Val, however, revealed an increased risk for carriers of the heterozygous Val654 allele (odds ratio $=$ $2.56,95 \%$ confidence interval $=1.08-6.08, P=0.028$ ).

Conclusion The rare Val654 is linked to the more frequent Val655, resulting in two consecutive valine residues instead of two isoleucine residues within the transmembrane domain. Computational analyses suggest that the Val654-Val655 allele provokes receptor dimerisation and activation, thus stimulating kinase activity and cell transformation. We hypothesise that ERBB2 Val654 represents an oncogenic variant that might, in addition, influence clinical outcome and predict worse prognosis. The occurrence of the Val654-Val655 haplotype in human breast cancer cells (MCF-7) supports the significance of our results.

Acknowledgement This manuscript has been accepted for publication [1].

Reference

1. Frank B, et al.: The rare ERBB2 variant lle654Val is associated with an increased familial breast cancer risk. Carcinogenesis 2005, 26:643-647.

\section{P1.19}

\section{Alpha-1 antitrypsin genotypes in breast cancer} patients

\section{Z El-Akawi, M Nusier, DH Sawalha}

Department of Biochemistry and Molecular Biology, Faculty of Medicine, Jordan University of Science and Technology, Irbid, Jordan Breast Cancer Research 2005, 7(Suppl 2):P1.19 (DOI 10.1186/bcr1106) Alpha-1 antitrypsin (1-AT) is a secretory glycoprotein mainly produced in the liver and monocytes. It is the most abundant serine protease inhibitor in human plasma. Proteolytic enzymes play a significant role in the expression of the malignant phenotype, including the loss of growth regulation, invasiveness and formation of metastases. Deficiency of 1-AT is an inherited disorder characterized by reduced serum level of 1-AT. Protease inhibitors $Z$ (PiZ) and protease inhibitors $S$ (PiS) are the most common deficient genotypes of 1-AT. The association of deficient 1-AT subtypes with several tumors such as primary liver carcinoma, lung cancer, bladder cancer and malignant hepatoma was reported. This study aimed to determine the incidence of 1-AT genotypes (PiZ and PiS) in breast cancer female patients. Blood samples were collected from 111 patients. DNA was isolated and the PCR technique was performed to amplify the regions contain the $Z$ and $S$ mutations in exon $\mathrm{V}$ and exon III, respectively. Genotyping of the $Z$ and $S$ alleles was performed by restriction fragment length polymorphism analysis using the Taq1 restriction enzyme. Our results demonstrated that $100 \%$ of the breast cancer patients were homozygous for the normal allele (PiMM) and no PiZ and PiS genotypes were found.

\section{P1.20}

\section{Association of NCOA3 (AIB1) polymorphisms with breast cancer risk}

B Burwinkel', M Wirtenberger'1, B Frank', R Klaes², RK Schmutzler ${ }^{3}$, E Grzybowska ${ }^{4}$, A Försti 1,5, JL Bermejo', P Bugert6, B Wappenschmidt ${ }^{3}$, D Butkiewicz', J Pamula4, W Pekala4, H Zientek4, D Mielzynska7, E Siwinska7, CR Bartram², K Hemminki ${ }^{1,5}$

${ }^{1}$ Division of Molecular Genetic Epidemiology, German Cancer Research Center, Heidelberg, Germany; 2 Institute of Human Genetics, University of Heidelberg, Germany; ${ }^{3}$ Department of Molecular Gynaeco-Oncology, Division of Gynaecology and Obstetrics, Clinical Center University of Cologne, Germany; ${ }^{4}$ Department of Tumor Biology, Center of Oncology, Maria Sklodowska-Curie Institute, Gliwice, Poland; ${ }^{5}$ Karolinska Institute, Department at Biosciences at Novum, Huddinge, Sweden; ${ }^{6}$ Institute of Transfusion Medicine and Immunology, Red Cross Blood Service of Baden-WürttembergHessia, University of Heidelberg, Faculty of Clinical Medicine, Mannheim, Germany; ${ }^{7}$ Department of Genetic Toxicology, Institute of Occupational Medicine and Environmental Health, Sosnowiec, Poland Breast Cancer Research 2005, 7(Suppl 2):P1.20 (DOI 10.1186/bcr1107) The nuclear receptor coactivator 3 (NCOA3, also known as $A I B 1)$ is a coactivator of nuclear receptors like the estrogen receptor. NCOA3 is overexpressed in $\sim 60 \%$ of primary human breast tumours, and high levels of $N C O A 3$ expression are associated with tamoxifen resistance and worse survival rate. In contrast, NCOA3 deficiency suppresses $v$-Ha-ras-induced breast cancer initiation and progression in mice. Here we analysed the influence of NCOA3 coding single nucleotide polymorphisms on breast cancer risk by performing a case-control study using a German and a Polish study population, and identified an association between NCOA3 polymorphisms and breast cancer. A joint analysis of the German and Polish study population revealed a significant protective effect for the $1758 \mathrm{G}>\mathrm{C}(\mathrm{Q} 586 \mathrm{H})$ and $2880 \mathrm{~A}>\mathrm{G}$ (T960T) variants. In addition, haplotype analysis showed a protective effect of the 1758C-2880A and 1758G-2880G haplotypes (odds ratio $=$ $0.79,95 \%$ confidence interval $=0.67-0.93, P=0.004$ ). Due to the impact of NCOA3 in anti-estrogen therapy resistance, these polymorphisms might also influence therapy outcome in breast cancer. Acknowledgements BB and MW contributed equally to this work. 


\section{P1.21}

\section{Polymorphisms in the CRK gene and their} association with breast cancer risk

\section{K Wagner', K Hemminki',2, E Grzybowska ${ }^{3}$, A Försti ${ }^{1,2}$}

${ }^{1}$ Division of Molecular Genetic Epidemiology, German Cancer Research Center, Heidelberg, Germany; ${ }^{2}$ Department of Biosciences at Novum, Karolinska Institute, Huddinge, Sweden; ${ }^{3}$ Department of Tumor Biology, Centre of Oncology, Maria Sklodowska-Curie Institute, Gliwice, Poland

Breast Cancer Research 2005, 7(Suppl 2):P1.21 (DOI 10.1186/bcr1108) Background Recent findings suggest an important influence of the GH1/IGF1 axis in the development of breast cancer. By binding to its receptor, IGF-1 stimulates numerous downstream signaling proteins resulting in the regulation of cell proliferation, differentiation and apoptosis [1]. One member of this pathway is CRK ( $\mathrm{v}$-crk sarcoma virus CT10 oncogene homolog), which is tyrosine phosphorylated upon IGF-1 stimulation. CRK has been found to be overexpressed in various human tumor tissues and cell lines [2]. However, little is known about alterations in the genomic sequence of this gene.

Methods We sequenced the promoter and the coding region of the CRK gene in a small sample set of 23 breast cancer samples. We confirmed a $\mathrm{C}$ to $\mathrm{A}$ polymorphism at nucleotide position 49 with a synonymous amino acid change at Arg17, which was in nearly $100 \%$ linkage to the promoter polymorphism C-289A, and we identified a novel polymorphic duplication of $22 \mathrm{bp}$ in the promoter region. In the further analyses we used a TaqMan allelic discrimination assay for the Arg17 polymorphism and a fluorescent fragment analysis to detect the duplication in a sample set of 352 Polish familial breast cancer cases and 485 matched controls. We determined the genotype and haplotype frequencies and calculated the odds ratios with $95 \%$ confidence intervals.

Results We did not observe any differences in the allele or genotype frequencies between the cases and controls for the duplication polymorphism. For the Arg17 polymorphism, the allele frequency of the A allele was slightly decreased among the cases compared with the controls $(52.3 \%$ vs $56.0 \%$, respectively), but the difference was not statistically significant. In the haplotype analysis, we observed a protective effect for the carriers of the Arg17 A and the duplication alleles (odds ratio $=0.17,95 \%$ confidence interval $=0.03-0.76$, $P=0.007$ ).

Conclusions CRK is a member of the GH1/IGF-1 pathway, whose members are often found to be overexpressed in human tumors. The, to our knowledge, novel 22 bp duplication in the promoter region results in multiplication of various putative transcription factor binding sites. This may lead to an altered expression of the CRK gene. In combination with the Arg17 A allele it showed a protective effect. The Arg 17 polymorphism was in nearly $100 \%$ linkage with a polymorphism in the promoter, which also might have an effect on transcription. However, a functional analysis is needed to investigate the effect of these polymorphisms on the expression.

References

1. Laban C, Bustin SA, Jenkins PJ: The GH-IGF-I axis and breast cancer. Trends Endocrinol Metab 2003, 14:28-34.

2. Nishihara H, Tanaka S, Tsuda M, Oikawa S, Maeda M, Shimizu M, Shinomiya $H$, Tanigami A, Sawa $H$, Nagashima K: Molecular and immunohistochemical analysis of signaling adaptor protein Crk in human cancers. Cancer Lett 2002, 180:55-61.

\section{P1.22}

\section{High-density screening of the Zbtb7 gene in breast cancer patients}

A Salas ${ }^{1,2}$, A Vega ${ }^{2,3}$, M Torres ${ }^{2}$, I Quintela ${ }^{2}$, C Phillips ${ }^{2}$, R Rodríguez-López ${ }^{4}$, G Rivas ${ }^{4}$, J Benítez ${ }^{4}$, A Carracedo ${ }^{1,2,3}$

1 Unidade de Xenética, Instituto de Medicina Legal, Facultad de Medicina, Universidad de Santiago de Compostela, Galicia, Spain; ${ }^{2}$ Centro Nacional de Xenotipado, Hospital Clínico Universitario, Santiago de Compostela, Galicia, Spain; ${ }^{3}$ Fundación Pública Galega de Medicina Xenómica, Hospital Clínico Universitario, Universidad de Santiago de Compostela, Galicia, Spain; ${ }^{4}$ Departamento de Genética Humana, Centro Nacional Investigaciones Oncológicas, Madrid, Spain Breast Cancer Research 2005, 7(Suppl 2):P1.22 (DOI 10.1186/bcr1109) It has been proposed that the excess of the familiar risk associated with breast cancer could be explaining by multiple weakly predisposing alleles. Hence there is much interest in the search for low-penetrance genes/variants for breast cancer, which exist with high prevalence in the general population. Maeda and colleagues [1] recently identified the transcriptional repressor FBI1, which they called Pokemon (POK erythroid myeloid ontogenic factor), as a critical factor in oncogenesis. This protein is codified by the ZBTB7 gene ('zing finger and BTB domain containing 7'; GenelD, 51341). Mouse embyronic fibroblasts lacking Zbtb7 were completely refractory to oncogene-mediated cellular transformation. Conversely, FBl1 overexpression led to overt oncogenic transformation both in vitro and in vivo in transgenic mice. FBI1 can specifically repress the transcription of the tumor suppressor gene ARF (600160). Maeda and colleagues [1] found that FBI1 is aberrantly overexpressed in human cancers, and its expression levels predict biologic behavior and clinical outcome. On the other hand, tissue microarray analysis (TMA) in breast carcinomas has revealed high levels of Pokemon expression in a subset of these tumors. In addition, the genomic region where the ZBTB7 gene resides (19p13.3) is a hotspot for chromosomal translocations [2]. ZBTB7 is therefore a good candidate for a breast cancer low-penetrance gene. We aim to determine whether common polymorphisms (frequency $\geq 10 \%)$ in the ZBTB7 gene are associated with breast cancer risk in Spanish patients. A set of 22 validated binary SNP polymorphisms were selected from public databases (e.g. HapMap) and private databases (e.g. Celera and RealSNP) according to criteria of coverage (average $1 \mathrm{SNP} / 1.7 \mathrm{~kb}$ ). These SNPs cover the flanking regions $(10,000 \mathrm{bp})$, including the promoter region, introns, and coding nonsynonymous SNPs. About 550 cases and 550 controls have been genotyped. Genotyping was performed using the MassARRAY SNP genotyping system (Sequenom Inc., San Diego, CA, USA).

To our knowledge, this is the first time that the ZBTB7 gene has been analyzed in breast cancer patients. The results will be presented at the time of the congress and will be interpreted in the light of the worldwide population study shown in another presentation from the same authors.

Acknowledgments This work was supported by grants from the Ministerio de Sanidad y Consumo (Fondo de Investigación Sanitaria; Instituto de Salud Carlos III, PI030893; SCO/3425/2002) and Genoma España (CeGen; Centro Nacional de Genotipado; Nodo Santiago de Compostela).

\section{References}

1. Maeda T, Hobbs RM, Merghoub T, Guernah I, Zelent A, CordonCardo C, Teruya-Feldstein J, Pandolfi PP: Role of the protooncogene Pokemon in cellular transformation and ARF repression. Nature 2005, 433:278-285.

2. Cancer Genome Anatomy Project [http://cgap.nci.nih.gov/ Chromosomes] 


\section{P1.23}

ZBTB7 HapMap in a worldwide population study A Salas',2, A Vega ${ }^{2,3}$, C Phillips'2, M Torres², I Quintela2, A Carracedo $1,2,3$

1 Unidade de Xenética, Instituto de Medicina Legal, Facultad de Medicina, Universidad de Santiago de Compostela, Galicia, Spain; ${ }^{2}$ Centro Nacional de Xenotipado, Hospital Clínico Universitario,

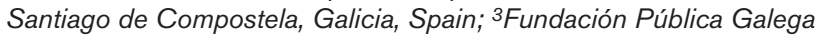
de Medicina Xenómica, Hospital Clínico Universitario, Universidad de Santiago de Compostela, Galicia, Spain

Breast Cancer Research 2005, 7(Suppl 2):P1.23 (DOI 10.1186/bcr1110) The ZBTB7 gene is aberrantly overexpressed in human tumours [1] and is an obvious candidate for a breast cancer low-penetrance gene. Here we aimed to analyze the SNP and haplotypic variability of the ZBTB7 gene in human populations. The information available in SNPs databases is still limited; for example, HapMap contains information of only four ethnic groups, two of them from East Asia [2,3]. The inference of the portability of tagSNPs using this limited amount of information is therefore still under debate. We have thus selected 22 validated polymorphisms covering the ZBTB7 gene and flanking regions using public and private SNP databases. There are more than 1200 worldwide DNA samples (40 human populations), which include those contained in the CEPH Genome Diversity Cell Line Panel. Genotyping was performed using the MassARRAY SNP genotyping system (Sequenom Inc., San Diego, CA, USA). Briefly, it involves multiplex PCR and minisequencing assays, designed with Spectro DESIGNER software (Sequenom Inc.), followed by mass spectrometry analysis with the Bruker Bi-flex MALDITOF mass spectrometer (Bruker Daltonics, Billerica, MA, USA). Spectral output was analyzed using SpectroTYPER-RT 3.1 software (Sequenom Inc.) and by manual review.

The present high-density SNP mapping study will facilitate a map of specific population variation and patterns of linkage disequilibrium at the ZBTB7 region in different human populations, and will facilitate the adequate selection of a highly efficient set of tag SNPs that will capture the bulk of the (potentially pathogenic) variation. We have found that patterns of LD and haplotype diversity at the ZBTB7 gene vary considerably among different populations. Thus, sub-Saharan African populations showed higher levels of haplotype diversity and shorter blocks, while non-Africans showed a higher level of LD and lower haplotype diversity, as expected according to population history. According to these patterns, we will discuss the efficiency of these LD patterns and tagSNPs to capture candidate SNPs at the ZBTB7 gene in tumor association studies.

Acknowledgements This work was supported by grants from the Ministerio de Sanidad y Consumo (Fondo de Investigación Sanitaria; Instituto de Salud Carlos III, PI030893; SCO/3425/2002) and Genoma España (CeGen; Centro Nacional de Genotipado; Nodo Santiago de Compostela).

References

1. Maeda T, Hobbs RM, Merghoub T, Guernah I, Zelent A, CordonCardo C, Teruya-Feldstein J, Pandolfi PP: Role of the protooncogene Pokemon in cellular transformation and ARF repression. Nature 2005, 433:278-285.

2. International HapMap Consortium: Integrating ethics and science in the International HapMap Project. Nat Rev Genet 2004, 5:467-475.

3. The International HapMap Consortium: The International HapMap Project. Nature 2003, 426:789-796.

\section{P1.24}

\section{A critical need for molecular markers of breast cancer risk and risk reduction KA Johnson, LG Ford}

Division of Cancer Prevention, National Cancer Institute, NIH, Bethesda, Maryland, USA

Breast Cancer Research 2005, 7(Suppl 2):P1.24 (DOI 10.1186/bcr1111) Carcinogenesis is a chronic disease process underlying the clonal evolution of cells progressing to the point of uncontrolled growth, metastatic potential, and molecular heterogeneity. By convention, chemoprevention drugs are developed from a molecular perspective with the goal of interrupting carcinogenesis before the occurrence of invasive lesions or extreme heterogeneity. The most successful demonstration of cancer chemoprevention to date has been an overall $49 \%$ reduction of invasive breast cancer, with a similar reduction in premalignant lesions by tamoxifen in the Breast Cancer Prevention Trial (BCPT) [1]. It is noteworthy that the main effect of tamoxifen is likely to be mediated through the estrogen receptor (ER), as reflected by a $70 \%$ reduction of lesions that are ER-positive in contrast to little or no effect on the incidence of ER-negative lesions. For interventions that are related to ER as a target, a number of drug development issues remain to be addressed including dose, schedule, and the comparative net clinical benefit of various selective ER modulators versus pure antiestrogens, aromatase inhibitors, and combinations. In the BCPT there were 13,388 participants, and there were more than 22,000 in the STAR trial. The large sample size that may be needed for a randomized clinical trial to observe a prevention effect severely limits the opportunity to explore a multiplicity of important questions in clinical chemoprevention.

Molecular studies have been helpful in classifying breast cancers according to categories of response to intervention. For instance, cytogenetic studies combined with molecular profiling suggest that ERfocused interventions are likely to address a particular subset of tumors arising from the luminal cell population [2]. As tumor subsets become better characterized, the need for additional prevention studies can be anticipated to address larger subsets (e.g. a combination of drugs for overlap) versus smaller subsets of individuals at risk. In order to reduce the sample size of future prevention trials, new molecular approaches are needed. One strategy would be to use non-invasive molecular tests to identify individuals at increased breast cancer risk so that populations for prevention trials could be further enriched according to that risk. Of the approaches currently under investigation, proteomic studies theoretically offer an opportunity to improve risk identification. Investigators who are performing proteomic studies for early detection are encouraged to expand their investigations to see whether it is possible to delineate according to ER status and between non-invasive conditions such as hyperplasia and DCIS versus invasive cancer.

Another strategy for increasing the efficiency of breast cancer prevention trials is the validation of intermediate endpoint biomarkers [3] to secure validated intermediate endpoint biomarkers (VIEBs). If molecular entities in serum could be identified on the basis that they are predictably correlated with the future development of breast cancer, then a reduction in the VIEB level could serve as evidence of a preventive effect. Early work in this area suggests that nucleic acids in serum might be used to identify individuals with premalignant lesions [4].

Clinical correlation is needed for VIEBs and other molecular indicators of risk so that targets in addition to the ER can also be more efficiently studied. Targets of interest for breast cancer prevention include the EGFR family, RAR/RXR and mediators of inflammation or oxidative damage.

References

1. Fisher B, Costantino JP, Wickerham DL, et al.: J Nat/ Cancer Inst 1998, 90:1371-1388.

2. Sørlie T, Tibshirani R, Parker J, et al.: Proc Natl Acad Sci USA 2003, 100:8418-8423.

3. Schatzkin A, Freedman LS, Schiffman MH, et al.: J Natl Cancer Inst 1990, 82:1746-1752.

4. Gocke CD, Benko FA, Kopreski MS, et al.: Ann NY Acad Sci 2000, 906:44-50. 


\section{P2.01}

\section{Gene expression profiling in whole-blood samples from postmenopausal women exposed to hormone replacement therapy \\ V Dumeaux ${ }^{1,2}$, J Johansen', AL Børresen-Dale ${ }^{3}$, E Lund ${ }^{1}$}

1 Institute of Community Medicine, University of Tromso, Norway; ${ }^{2}$ Equipe E3N-EPIC, INSERM XR521, Institut Gustave-Roussy, Paris, France; ${ }^{3}$ The Norwegian Radium Hospital, Department of Genetics, Oslo, Norway

Breast Cancer Research 2005, 7(Suppl 2):P2.01 (DOl 10.1186/bcr1112) Background Accumulating evidence on postmenopausal hormone therapy confirms the deleterious effects on risk of breast cancer or stroke and questions the positive effects on quality of life and coronary disease risk. A large-scale gene expression study may present promising new insights into the effect of hormone replacement therapy (HRT). Our study explores the gene expression profile from whole-blood total RNA, which is an important and relatively unexplored issue in human biology.

Methods In early autumn 2003, 500 women participating in the Norwegian Women and Cancer (NOWAC) cohort were randomized and invited to give blood samples (393 returned the questionnaire). Blood samples were collected in a PAXgene tube in late autumn 2003 and a short questionnaire additional to those previously given in the NOWAC study was completed. In our study, 100 women (50 HRT users and 50 non-HRT users) born between 1943 and 1949 with normal body mass index $\left(18.5<\mathrm{BMl}<29.9 \mathrm{~kg} / \mathrm{m}^{2}\right)$ and no other medication use were selected. After extraction amplification and labelling of the samples, we hybridized them overnight to Agilent Human 1A oligoarrays (G4110b; Agilent Technologies, Palo Alto, CA, USA) containing 22,153 features representing 20,173 unique genes.

Results Genes identified by $t$ test with $P<0.03(n=253)$ were used to build a classifier using the nearest shrunken centroids method. Results did not reveal any distinct gene list that predicts accurately HRT exposure (error rate $=0.42$ ). We performed a new analysis including, among HRT users, only women who were using continuous combined treatment (ethinylestradiol and norethisterone acetate). The performance of the classifier (i.e. 98 genes) improved (error rate $=0.25$ ). The specificity $(78.7 \%)$ was slightly better than the sensitivity $(68.0 \%)$.

We then tested the significant changes in a single gene by different methods like the $t$ test, significance analysis of microarrays controlling for the false discovery rate and Bayesian ANOVA analysis, which balance the false discovery rate and the true positive rate. Only few expression alterations of minor magnitude caused by HRT could be detected in whole-blood total RNA. The weak signals of exposureinduced changes in whole blood made it very difficult, or even impossible, to identify single genes statistically significant with the background of thousands of individuals genes tested simultaneously. Instead, we focused on identifying significant pattern changes of biologic process in genes identified from the $t$ test, using the annotations defined by the Gene Ontology Consortium.

Conclusions Mixed cell types in whole blood made it more difficult to observe differences in gene expression profiles. According to the little amplitude of expression alterations observed in whole blood, large sample sizes are needed to conduct global expression profiling. Although one gene change may be small and difficult to detect accurately in a significant test, significant enrichments in the biologic process of genes with small changes after HRT use have been assessable.

\section{P2.02}

\section{Effects of oestrogen on gene expression in the} epithelium and stroma of the normal human breast AH Sims, KR Ong, CL Wilson, A Howell, RB Clarke

Breast Biology Group, Division of Cancer Studies, University of Manchester, Christie Hospital, Manchester, UK

Breast Cancer Research 2005, 7(Suppl 2):P2.02 (DOI 10.1186/bcr1113)

Background Oestrogen (E2) is central to the development of breast cancer, and anti-oestrogens have been shown to reduce the risk of the disease. However, little is known about the effect of E2 on the normal human breast, particularly when the epithelium and stroma are intact. Previous expression profiles of the response to E2 were performed on tumour cell lines, in the absence of stroma [1-3].

Methods We investigated gene expression in normal human breast tissue (removed from benign lesions in premenopausal women) transplanted into 9-week-old to 10-week-old athymic nude (Balb/c/nu/nu) mice.

Transplantation was performed in order to obviate the potential effects of the phase of the menstrual cycle. After 2 weeks, when proliferation and progesterone receptor (PR) expression are minimal, the mice were treated with E2 using a $2 \mathrm{mg}$ slow-release pellet for 1 week, which we have previously shown to be optimal for inducing proliferation and PR [4]. At completion of treatment, three tissue samples were generated from each of the six original normal breast tissue samples - two of which were untreated and one of which had been treated with oestrogen. RNA was isolated from each of these samples, then labelled and hybridised to Affymetrix HG-U133A (human) chips on which 22,283 genes are represented. RMA and MAS5 normalisation methods were utilised with bioconductor analysis software.

Results Oestrogen treatment was found to be the major source of variation in gene expression. Our study shows that known E2responsive genes such as trefoil factor 1 (pS2) and amphiregulin are also differentially expressed due to oestrogen treatment of normal breast tissue. In addition, many of the genes that showed the greatest responses to E2 have previously been suggested as independent breast cancer prognostic or diagnostic markers (including mammaglobin, prolactin-inducing peptide and keratin 19).

Conclusion We report the first global gene expression study to look at the effects of oestrogen on the epithelium and stroma of normal human breast tissue, which may give clues to the paracrine action of oestrogen in proliferation. These data form the basis for efforts towards the detection of early gene expression changes leading to breast cancer development.

References

1. Coser KR, et al:: Global analysis of ligand sensitivity of estrogen inducible and suppressible genes in MCF7/BUS breast cancer cells by DNA microarray. Proc Natl Acad Sci USA 2003, 100:13994-13999.

2. Frasor J, et al.: Profiling of estrogen up- and down-regulated gene expression in human breast cancer cells: insights into gene networks and pathways underlying estrogenic control of proliferation and cell phenotype. Endocrinology 2003, 144:4562-4574.

3. Inoue $A$, et al.: Development of cDNA microarray for expression profiling of estrogen-responsive genes. $J \mathrm{Mol}$ Endocrinol 2002, 29:175-192.

4. Clarke RB, Howell A, Anderson E: Estrogen sensitivity of normal human breast tissue in vivo and implanted into athymic nude mice: analysis of the relationship between estrogen-induced proliferation and progesterone receptor expression. Breast Cancer Res Treat 1997, 45:121-133.

\section{P2.03}

\section{Mammary development fate and breast cancer risk D Medina}

Department of Molecular and Cellular Biology, Baylor College of Medicine, Houston, Texas, USA

Breast Cancer Research 2005, 7(Suppl 2):P2.03 (DOI 10.1186/bcr1114) A full-term pregnancy or a 3-week treatment with estrogen and progestins induces a protective state against chemical carcinogeninduced mammary tumorigenesis in rats and mice. These experimental models are a paradigm for the well-established fact that an early fullterm pregnancy in humans induces a life-long decreased risk for breast cancer. Up to now, this hypothesis has not been successfully tested in non-carcinogen-treated rodents. We tested the hypothesis in p53 null mouse mammary epithelium. A 2-week exposure to estrogen and progesterone reduced significantly $(P<0.05)$ the incidence of spontaneous breast cancer in p53 null epithelial cells. The hormone- 
treated cells had a unique gene expression profile at 40 weeks post hormone removal compared with untreated p53 null epithelial cells. Current experiments are examining the developmental stage specificity of the response to estrogen and progesterone. Additionally, genes specific to the hormone-treated p53 null cells are being further evaluated. These results indicate that short periods of hormone treatment can markedly delay mammary tumorigenesis in models where the initiating oncogene is relevant to human breast cancer.

\section{P2.04}

BRCA1 directly modulates gene expression required for estrogen biosynthesis: a possible mechanism of tissue-specific tumor suppression

\section{Y Hu', S Aiyar', W Yue ${ }^{2}$, S Ghosh'1, Y Lu', R Li ${ }^{1}$}

${ }^{1}$ Department of Biochemistry and Molecular Genetics, and

${ }^{2}$ Department of Medicine and Division of Endocrinology, School of Medicine, University of Virginia, Charlottesville, Virginia, USA

Breast Cancer Research 2005, 7(Suppl 2):P2.04 (DOI 10.1186/bcr1115) Background Mutations in the tumor suppressor gene BRCA1 are associated with an elevated risk of breast and ovarian cancer. The tissuespecific nature of BRCA1 mutation-associated tumors stands in stark contrast to the diverse array of nuclear functions mediated by the wildtype BRCA1. These functions include regulation of transcription and the DNA damage response. While the DNA repair function of BRCA1 clearly contributes to overall genetic stability, loss of BRCA1 function in DNA repair, a process that is universally important to all cell types in both genders, cannot easily explain why BRCA1 mutations predominantly affect breast and ovary, two major estrogen-responsive tissues in women. We hypothesize that BRCA1 confers tissue-specific tumor suppression by directly modulating expression of a set of breast-cancer-related genes. Methods and results Aromatase, which catalyzes conversion from androgen to estrogen, is a rate-limiting enzyme in estrogen biosynthesis and is a key player in breast cancer development. Aromatase is specifically expressed in ovarian granulosa cells and several peripheral tissues via the actions of multiple tissue-specific promoters. In premenopausal women, expression of aromatase in ovarian granulosa cells dictates levels of circulating estrogen. In many postmenopausal breast cancer and endometrial malignancy tissues, the in situ aromatase production is significantly elevated. Here, we show that the cyclic AMP-dependent expression of aromatase is inversely correlated with the protein level of BRCA1 in human ovarian granulosa cells. Importantly, transient knockdown of BRCA1 enhances the expression of aromatase in both ovarian granulosa cells and cells from breast tissue. Chromatin immunoprecipitation indicates that endogenous BRCA1 is preferentially associated with a tissue-specific transcriptional promoter of the aromatase gene. Thus, BRCA1 negatively controls estrogen biosynthesis by repressing aromatase expression, providing a novel and logical molecular explanation for the tissue-specificity conundrum in BRCA1 biology.

Conclusion and significance The notion that BRCA1 confers tissuespecific tumor suppression in an endocrine fashion is also consistent with results from recent clinical and animal studies. In particular, prophylactic oophorectomy reduces the risk of both breast cancer and ovarian cancer in carriers of $B R C A 1$ mutations [1,2], suggesting that circulating estrogen in premenopausal women may contribute to the development of BRCA1-associated tumors. Moreover, tissue-specific knockout of Brca1 in mouse ovarian granulosa cells induces tumors in the ovaries and uterine horns. Importantly, these tumors still carry wildtype alleles of Brca1 [3]. Given that aromatase inhibitors are one of the most efficacious endocrine agents in breast cancer treatment, the link between BRCA1 and aromatase expression may lead to novel therapies for both prevention and treatment of breast cancer.

Acknowledgement This work was funded by grants from the National Institutes of Health and Department of Defense Breast Cancer Research Program.

\section{References}

1. Kauff ND, et al:: N Engl J Med 2002, 346:1609-1615.

2. Rebbeck TR, et al.: N Engl J Med 2002, 346:1616-1622.

\section{P2.05}

HER2 and ER $\beta 2$ downregulate estrogen-responsive element-mediated transcription activity of ER $\alpha$ positive cells in response to estrogen stimulation B-W Park', M-K Heo'2, S-O Park ${ }^{2}$, S-I Kim', K-S Kim³ , C-H Park ${ }^{1}$ Department of Surgery, ${ }^{2}$ Brain Korea 21 Project and ${ }^{3}$ Department of Biochemistry and Molecular Biology, Yonsei University College of Medicine, Seoul, Korea; ${ }^{4}$ Hallym University College of Medicine, Seoul, Korea

Breast Cancer Research 2005, 7(Suppl 2):P2.05 (DOI 10.1186/bcr1116) Background Although HER2 expression is more common in ERnegative and PR-negative breast cancers, HER2 overexpression is observed in ER-positive cancers and these cancers seem to have intrinsic resistance to endocrine treatment. There are controversial reports about the effect of HER2 on estrogen dependence. ER $\beta 2$ has been reported to act as a dominant-negative regulator of ER $\alpha$ in the breast, and ER $\beta 1$ and/or ER $\beta 2$ might be co-expressed with ER $\alpha$ in breast cancer. It is therefore necessary to investigate the effect of HER2 and ER $\beta 2$ expression on the ERE-mediated transcription activity in the cells co-expressing ER $\alpha$.

Methods NIH3T3 cells, T6-17 cells (NIH3T3 cells stably transfected with HER2), and MCF-7 cells were maintained in dextran-coated charcoal-stripped 10\% Dulbecco's modified Eagle medium. Transient transfection of constructs ( $p c D N A 3-E R \alpha$, pcDNA3-ER $\beta 2$, pERE-luc, pcDNA-HER2) into each cell was performed using the Lipofectamine PLUS $^{\mathrm{TM}}$ system. The reporter gene assay using estrogen-responsive element (ERE)-luciferase was used to measure the ER $\alpha$ transcriptional activity after treatment of $17-\beta$-estradiol and tamoxifen.

Results The reporter gene assay using ERE-luciferase showed much less responsiveness to estrogen in HER2-overexpressing T6-17 cells than in NIH3T3 cells, but there was no remarkable difference after treatment of tamoxifen. The responsiveness to estrogen in HER2transfected MCF-7 cells was a little less, but not remarkable, than that in control MCF-7 cells. However, the responsiveness to estrogen in MCF-7 cells was decreased in a dose-dependent manner of HER2 expression. ERE-mediated transcriptional activity was decreased with the increase of ER $\beta 2$ expression; it is much more remarkable in HER2overexpressing T6-17 cells.

Conclusion Expression of HER2 and/or ER $\beta 2$ reduces estrogen responsiveness in ER $\alpha$-positive tumor cells. HER2 might be associated with growth dependence of the tumor cells, but ER $\beta 2$ seems to be associated with binding affinity to estrogen and DNA.

\section{P2.06}

\section{The anti-estrogen ICI 182,780, but not tamoxifen,} inhibits the growth of MCF7 breast cancer cells refractory to long-term estrogen deprivation through downregulation of ER and IGF signalling L-A Martin', S Pancholi ', I Farmer', SRD Johnston' ${ }^{2}$, M Dowsett ${ }^{1}$ ${ }^{1}$ Academic Department of Biochemistry and The Breakthrough Toby Robins Breast Cancer Research Centre, Institute of Cancer Research, London, UK; ${ }^{2}$ The Breast Unit, Royal Marsden Hospital, London, UK Breast Cancer Research 2005, 7(Suppl 2):P2.06 (DOI 10.1186/bcr1117) Long-term culture of MCF-7 wild-type cells in steroid-depleted medium (long-term estrogen deprivation [LTED]) results in hypersensitivity to estradiol (E2) coinciding with elevated levels of ER $\alpha$, and enhanced type I receptor tyrosine kinase growth factor signalling. In this study we aimed to compare the effects of the pure anti-estrogen ICl 182,780 (ICl) with the competitive anti-estrogen tamoxifen (TAM) on estrogen and IGF signalling in these cells. Wild-type MCF-7 and LTED cells were treated with a seven-log concentration range of E2, TAM or ICl. Effects on cell growth, ER $\alpha$ transactivation, expression of $E R \alpha, E R \beta$, and components of the IGF pathway were measured in the presence of or the absence of insulin. In the presence of insulin, growth of LTED cells was refractory to TAM but was inhibited by $\mathrm{ICl}$ and $\mathrm{E} 2$. In the absence of insulin, LTED cells showed persistent hypersensitivity to 
E2, and remained inhibited by ICl but largely unaffected by TAM. Most noteworthy in the absence of insulin, we revealed that E2 doses in excess of $10^{-10} \mathrm{M}$ were inhibitory for the LTED cells leading to enhanced apoptosis. ICl but not TAM inhibited ER-mediated gene transcription and resulted in a concomitant dose-dependent reduction in ER $\alpha$ levels, while having no effect on ERB. As previous studies have suggested that endocrine resistance may result from the non-classical interaction of ER $\alpha$ with AP-1, we transfected the wild-type and LTED cells with an AP-1 reporter construct and monitored activity in response to E2, TAM and $\mathrm{ICl}$. No alteration was detected, suggesting that in this setting transcriptional activity via the ER remained wholly or partially classical via a direct ER/ERE interaction. Analysis of the expression level of proteins within the IGF pathway plus or minus insulin revealed elevated levels of IGF-1 receptor and insulin receptor substrate 2 in LTED versus the wild-type MCF- 7 cells, and ICI but not TAM reduced their expression in a dose-dependent fashion. IGF signalling, as well as ER $\alpha$ expression and function, are thus enhanced during LTED. While the resultant cells were resistant to TAM, ICl downregulates ER $\alpha$, reducing IGF signalling and cell growth. These results support the use of $\mathrm{ICl}$ in women with ER-positive breast cancer who have relapsed on an aromatase inhibitor.

\section{P2.07}

\section{Molecular prediction of tamoxifen resistance in breast cancer}

M Kok, TMC van den Berg, LJ Delahaye, A Floore, AM Glas, JL Peterse, LFA Wessels, LJ van 't Veer, SC Linn

The Netherlands Cancer Institute, Amsterdam, The Netherlands

Breast Cancer Research 2005, 7(Suppl 2):P2.07 (DOI 10.1186/bcr1118)

Background Estrogen receptor (ER) alpha-positive breast cancer patients are commonly treated with tamoxifen, a potent and widely used anti-estrogen. However, only one-half of the recurrences of ERpositive breast tumors respond to tamoxifen while the other half is resistant [1]. The ability to accurately predict tamoxifen treatment outcome would therefore significantly advance the management of breast cancer. The aim of the current project is to identify a gene expression profile associated with tamoxifen resistance using microarray analysis.

Patients and methods To identify gene expression patterns that might predict response to tamoxifen, 90 breast cancer patients were selected for whom fresh frozen tissue was available. All these patients had received surgery with or without radiotherapy for primary breast cancer, while none had received adjuvant systemic treatment. All patients developed metastatic disease and were subsequently treated with tamoxifen. Response was mainly determined radiographically [2]. Approximately $40 \%$ of these patients had CR/PR or clinical benefit during less than 6 months and are defined as tamoxifen resistant, whereas the remaining $60 \%$ are defined as tamoxifen sensitive (CR/PR or clinical benefit for more than 6 months). Of the latter group, 17 out of 54 even showed no progression for at least 2 years.

Gene expression profiling was performed using $44 \mathrm{~K}$ oligo microarrays. Data analysis is ongoing and results will be presented at the MBBC symposium.

Future directions $\mathrm{A}$ validation set will be analyzed to confirm our initial findings. Furthermore, we will test whether this profile can also be used in the adjuvant setting. In addition, we will evaluate the combination of microarray analysis and targets identified by RNAi screens in vitro in determining diagnostic tools for prediction of therapy outcome.

References

1. Pritchard Kl: Endocrine therapy of advanced disease: analysis and implications of the existing data. Clin Cancer Res 2003, 9: 460S-467S.

2. Hayward JL, Carbone PP, Heuson JC, Kumaoka S, Segaloff A, Rubens RD: Assessment of response to therapy in advanced breast cancer: a project of the Programme on Clinical Oncology of the International Union Against Cancer, Geneva, Switzerland. Cancer 1977, 39:1289-1294.

\section{P2.08}

The interaction of the ER with ERBB2 and PI3K results in elevated levels of AKT and p90RS in tamoxifen-resistant MCF-7 cells

S Pancholi ${ }^{1}$, A Lykkesfeldt ${ }^{2}$, SRD Johnston ${ }^{3}$, M Dowsett ${ }^{1}$, L-A Martin'

${ }^{1}$ Academic Department of Biochemistry and The Breakthrough Toby Robins Breast Cancer Research Centre, Institute of Cancer Research, London, UK; ${ }^{2}$ Danish Institute of Cancer Research, Copenhagen, Denmark; ${ }^{3}$ The Breast Unit, Royal Marsden Hospital, London, UK Breast Cancer Research 2005, 7(Suppl 2):P2.08 (DOI 10.1186/bcr1119) Despite advances in endocrine therapies, the majority of patients receiving tamoxifen will eventually relapse while retaining functional estrogen receptors. We have previously shown, using an MCF-7 cell line $\left(\mathrm{Tam}^{\mathrm{R}}\right)$ resistant to the anti-proliferative effects of tamoxifen, that elevated levels of phosphorylated AKT and p90RSK lead to an apparent ligand-independent phosphorylation of ER $\alpha \operatorname{ser}^{167}$. Analysis of the growth factor receptors in these cells indicated elevated levels of both phosphorylated ERBB2 and total ERBB3, which we postulated formed heterodimers and activated the PI3 kinase pathway leading to elevated AKT. However, our recent data suggest that elevated AKT results from an interaction between ER $\alpha$ and ERBB2. This association is knocked out by treatment with the pure anti-estrogen ICI 182,780 and is absent in the WT parental cell line. Similarly, we have demonstrated an association between the p85 subunit of PI3K and ER $\alpha$ in Tam ${ }^{R}$ but not in WT cells. Treatment of the cell lines with the specific AKT inhibitor SH6 and the MEK1/2 inhibitor U0126 caused greater decrease in cell proliferation and concomitant ER $\alpha$-directed transactivation in the $\operatorname{Tam}^{\mathrm{R}}$ cells versus the WT, confirming that these pathways are integral to the $\mathrm{Tam}^{\mathrm{R}}$ phenotype. To establish whether $\mathrm{p} 90^{\mathrm{RSK}}$ or AKT was responsible for the phosphorylation of the estrogen receptor at $\operatorname{ser}^{167}, \operatorname{Tam}^{R}$ and WT cells were treated with $\mathrm{SH} 6$, U0126 or a combination of the two. Blocking either pathway individually had little effect on ER $\alpha$ ser167 phosphorylation. However, a combination of the two inhibitors resulted in almost complete loss of phosphorylation. These data were confirmed using siRNA technology to suppress MAPK and AKT expression. Taken together these data suggest that, in this setting, the ER functions via a non-genomic mechanism, associating with ERBB2 and $\mathrm{PI} 3 \mathrm{~K}$ at the cell membrane leading to activation of both $\mathrm{p} 90^{\mathrm{RSK}}$ and AKT. This in turn leads to phosphorylation of ER $\alpha$ ser $^{167}$, ultimately regulating cell growth via genomic mechanisms. Although several of these complexes have previously been postulated, to our knowledge this is the first demonstration of this phenomenon in a tamoxifenresistant cell line.

\section{P2.09}

Establishment and characterization of two breast cancer xenografts in immunodeficient mice for studies on hormone-dependent and hormoneindependent tumor growth, progression and invasion $O$ Engebraaten, GO Hjortland, M Martinsen, T Halvorsen, $\varnothing$ Fodstad, AH Ree

The Norwegian Radium Hospital and The Medical Faculty, University of Oslo, Norway

Breast Cancer Research 2005, 7(Suppl 2):P2.09 (DOI 10.1186/bcr1120)

Background Breast cancer studies in cell culture and in animals have been performed using a limited number of well-characterized, but old, cell lines and xenograft lines. With the aim of developing new breast cancer model systems, particularly for in vivo biology studies related to hormone-dependent and hormone-independent tumor growth, progression and invasion, and for use in experimental therapy studies, we have collected and implanted biopsy tissues from patients operated for mammary carcinomas in immunodeficient animals.

Methods Primary tumor tissue from 30 patients with breast cancer (staged as T2 tumors) was harvested and implanted in SCID mice, in a subcutaneous pocket containing Matrigel. All mice were implanted with 
continuous-release estrogen pellets. The tumors were transferred when reaching a size of approximately $15 \mathrm{~mm}$, to new animals using the same technique. Tumor tissue was harvested for further morphological and molecular characterization from passage six.

Results Of 32 tumors implanted into mice, two gave rise to viable tumors beyond passage four. One of these proved to be estrogen receptor alpha $(E R \alpha)$-positive and the other $E R \alpha$-negative. Both showed characteristic epithelial cell morphology. Separate experiments showed that even the ER $\alpha$-negative tumor was growth accelerated in the estrogen-supplemented animal. RNA expression analysis confirmed the ER $\alpha$ status in the tumors. Furthermore, the expression of the estrogen-regulated genes TFF1 and CCND1 were high in the ER $\alpha$ positive tumor but absent in the ERo-negative tumor. Although described as being ER $\alpha$ regulated, the VEGF transcript was expressed in the ER $\alpha$-negative tumor, but not in the ER $\alpha$-positive tumor. A difference between the tumors was also seen in the expression of other genes relevant to the tumor growth, progression and invasion, as CDKN1A, COM1, TIMP-1 and MMP-14 demonstrated a high expression in the ER $\alpha$-negative tumor and a low or missing expression in the ER $\alpha$-positive tumor. The expression of CDK4, TIMP-2 and MMP-2 was similar in the two different tumors.

Conclusion As in other studies, the establishment of breast cancer xeonografts is difficult, with a success rate below $10 \%$. However, the two established breast cancer xenograft lines have different characteristics and show marked differences in gene expression patterns that can be related to their ER $\alpha$ status. Further studies and characterization have been initiated using RNA expression microarray analysis.

\section{P2.10}

\section{Sex-hormone binding globulin receptor-mediated growth inhibition in breast cancer cells: a proteomics approach}

\section{VM Bowers-Morrow, SO Ali}

Department of Biological Sciences, Macquarie University, Sydney, Australia

Breast Cancer Research 2005, 7(Suppl 2):P2.10 (DOI 10.1186/bcr1121) Background Both estrogen (E2) and growth factors stimulate proliferation of E2-dependent breast tumor cells. Functional cross-talk exists between E2-directed and growth factor-directed pathways. Convergence between these pathways may lead to a synergistic feedforward circuit, resulting in a stronger or more sustained proliferative response in breast cancer cells [1]. Another signaling pathway involved in the modulation of breast cancer cell proliferation is mediated by the receptor for sex hormone-binding globulin (SHBG-R). SHBG has been found to function as part of a novel steroid signaling system that is independent of the 'classical pathway' for intracellular steroid receptors. This 'alternative pathway' (Fig. 1) involves the activation of membrane SHBG-Rs by SHBG and E2. It has been demonstrated that SHBG, through CAMP and protein kinase A (PKA), can inhibit the proliferative effect of E2 on breast cancer cells [2-4]. Its role in E2dependent cancer cell proliferation may in the longer term be exploited for therapeutic strategies. The pathways involved have not yet been elucidated. Indeed, the receptor has not yet been identified, although it is known to exist from binding kinetics studies.

Methods We are applying proteomics techniques to identify the plasma membrane SHBG-R, and to elucidate the key signaling proteins involved in pathway(s) mediated by SHBG/SHBG-R binding. The following cell lines are the source material for membrane and cytosolic preparations: MCF-7, estrogen-dependent (estrogen receptor positive $[E R+]$ ) breast cancer cultured cells; MDA-MB 231, estrogeninsensitive (ER-) breast cancer cultured cells; MCF-10A, nonneoplastic mammary cells [control]. The steps for identification of SHBG-R are as follows. MCF-7 cells are known to have SHBG-R (a transmembrane protein) on their surfaces. Cell membranes are prepared from MCF-7 cells and then, following extraction, the membrane proteins are separated via two-dimensional electrophoresis

\section{Figure 1}

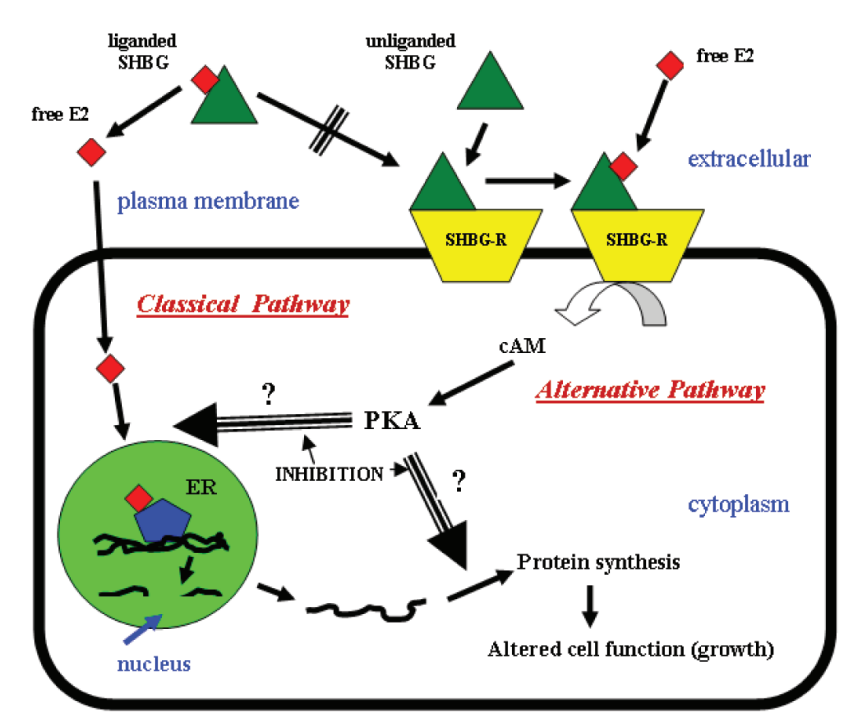

Model for SHBG-mediated signaling. The plasma membrane binding site for SHBG is preferentially expressed in ER+ cells with a reduced proliferative index [2-4]. SHBG-R only binds steroid-free or unliganded SHBG. Following binding of unliganded SHBG to SHBG-R on the cell membrane, E2 binds to the SHBG-SHBG-R complex, thereby activating it. Activation induces the synthesis of CAMP, which, in turn, triggers downstream signaling via PKA [2-4]. The biological outcome of this signaling pathway in cells of the human breast carcinoma cell line MCF-7 is decreased E2-mediated cell growth.

\section{Figure 2}

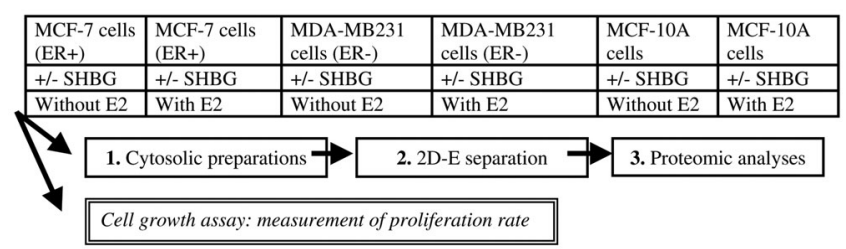

Identification of key signaling components of SHBG-mediated pathway(s). A flow-chart of the experimental design and method (involving comparative proteomic analyses) for identification of key regulated signaling components in SHBG-mediated pathways in breast cancer cells. Each cell type will be exposed to SHBG (vs not exposed to SHBG), and to human estradiol (E2) (vs not exposed to E2). Differential analysis of proteomes for the different cell lines/treatments will then be carried out. Differences in expression/post-translational modifications of specific proteins will determine what proteins are of interest. End effects will be examined as well as the results of time-dependent experiments.

binding assay using SHBG as the primary binder followed by antiSHBG (to detect bound SHBG) then a tertiary antibody conjugated to an enzyme system. Identification and partial characterization of SHBG$\mathrm{R}$ involves peptide mass fingerprinting and sequencing of amino acids, and international database searching. The design of experiments for identification of signaling components of SHBG-mediated pathway(s) is as shown in Fig. 2.

Results Progress will be reported on the identification and characterization of the SHBG receptor. 
Acknowledgement The authors wish to acknowledge a Macquarie University research grant.

References

1. Shupnik MA: Crosstalk between steroid receptors and the cSrc-receptor tyrosine kinase pathways: implications for cell proliferation. Oncogene 2004, 23:7979-7989.

2. Rosner W: The functions of corticosteroid-binding globulin and sex hormone-binding globulin. Endocr Rev 1990, 11:80-91.

3. Fortunati N, Fissore F, Fazzari A, Becchis M, Comba A, Catalano $M$, Berta L, Frairia R: Sex steroid binding protein exerts a negative control on estradiol action in MCF-7 cells (human breast cancer) through cyclic adenosine $3^{\prime}, 5^{\prime}$-monophosphate and protein kinase A. Endocrinology 1996, 137:686-692.

4. Fortunati $N$, Becchis $M$, Catalano MG, Comba A, Ferrera $P$, Raineri M, Berta L, Frairia R: Sex hormone binding globulin, its membrane receptor, and breast cancer: a new approach to the modulation of estradiol action in neoplastic cells. J Steroid Biochem Mol Biol 1999, 69:473-479.

\section{P2.11}

Identification of molecular apocrine breast tumours by microarray analysis

P Farmer ${ }^{1,2}$, H Bonnefoi ${ }^{3,4,5}$, V Becette, ${ }^{6}$ M Tubiana-Hulin ${ }^{6}$, P Fumoleau $^{7}$, D Larsimont ${ }^{8}$, G MacGrogan 9 , J Bergh ${ }^{10}$, D Cameron 11, D Goldstein 1,2, S Duss ${ }^{2}$, A-L Nicoulaz ${ }^{2}$, M Fiche ${ }^{12}$, C Brisken ${ }^{2}$, M Delorenzi $^{1}{ }^{12}, \mathbf{R}$ Iggo $^{2}$

${ }^{1}$ Swiss Institute of Bioinformatics, Lausanne, Switzerland; ${ }^{2}$ National Centre of Competence in Research Molecular Oncology, Swiss Institute for Experimental Cancer Research, Epalinges, Switzerland; ${ }^{3}$ Hôpitaux Universitaires de Genève, Geneva, Switzerland; ${ }^{4}$ Swiss Group for Clinical Cancer Research, St Gallen, Switzerland; ${ }^{5}$ European Organization on Research and Treatment of Cancer, Brussels, Belgium; ${ }^{6}$ Centre René Huguenin, St-Cloud, France; ${ }^{7}$ Centre René Gauducheau, Nantes, France; ${ }^{8}$ Institut Jules Bordet, Brussels, Belgium; 9/nstitut Bergonié, Bordeaux, France; ${ }^{10}$ Swedish Breast Cancer Group, Stockholm, Sweden; ${ }^{11}$ Anglo-Celtic Cooperative Oncology Group, Edinburgh, UK; ${ }^{12}$ Centre Hospitalier Universitaire Vaudois, Lausanne, Switzerland

Breast Cancer Research 2005, 7(Suppl 2):P2.11 (DOI 10.1186/bcr1122) Previous microarray studies on breast cancer identified multiple tumour classes, of which the most prominent, named luminal and basal, differ in expression of the estrogen receptor alpha gene (ER). We report here the identification of a group of breast tumours with increased androgen signalling and a 'molecular apocrine' gene expression profile. Tumour samples from 49 patients with large operable or locally advanced breast cancers were tested on Affymetrix U133A gene expression microarrays. Principal components analysis and hierarchical clustering split the tumours into three groups: basal, luminal, and a group we call molecular apocrine. All of the molecular apocrine tumours have strong apocrine features on histological examination $(P=0.0002)$. The molecular apocrine group is androgen receptor-positive (AR+) and contains all of the ER-negative tumours outside the basal group. Kolmogorov-Smirnov testing indicates that oestrogen signalling is most active in the luminal group, and androgen signalling is most active in the molecular apocrine group. ERBB2 amplification is more common in the molecular apocrine group than the other groups. Genes that best split the three groups were identified by the Wilcoxon test. Correlation of the average expression profile of these genes in our data with the expression profile of individual tumours in four published breast cancer studies suggest that molecular apocrine tumours represent $8-14 \%$ of tumours in these studies. Our data show that it is possible with microarray data to divide mammary tumour cells into three groups based on steroid receptor activity: luminal (ER+ AR+), basal (ERAR-) and molecular apocrine (ER- AR+).

\section{P3.01}

\section{Gene expression profiling to identify parity-induced changes in the human mammary gland} I Verlinden, N Güngör, J Janssens, L Michiels

Biomedisch Onderzoeksinstituut, Limburgs Universitair Centrum, School for Life Sciences, Transnational University Limburg,

Diepenbeek, Belgium

Breast Cancer Research 2005, 7(Suppl 2):P3.01 (DOI 10.1186/bcr1123)

Background The protective effect of an early first full-term pregnancy in relation to breast cancer risk is well established, but the molecular and cell-specific changes in the human mammary gland remain unclear. The search for these early predictive markers for breast cancer risk is of high priority because these markers could identify women at increased risk and could monitor preventive strategies.

Methods To identify the molecular changes associated with pregnancy-induced differentiation, we analysed the global gene expression profiles of normal mammary tissues from both a parous and a nulliparous woman, using serial analysis of gene expression (SAGE) [1]. This approach allowed us to identify sets of genes, known and unknown, that are differentially expressed in parous versus agematched nulliparous mammary gland tissues. Pair-wise comparisons of each tag were carried out in both SAGE libraries.

Results The normal mammary gland of a multiparous woman is characterised by several known differentiation markers such as casein $\kappa$, casein $\beta$, keratin 14, CCAAT/enhancer binding protein $\beta$ and $\delta$, and adipsin. Candidate genes involved in cyto-architectural remodeling and growth inhibition with a potential role in pregnancy-induced protection against breast cancer were also observed.

Several of the genes that are highly expressed in the nulliparous mammary gland and that are lost after pregnancy encode for growthpromoting, cytoskeletal and extracellular matrix proteins. One of these genes, the small breast epithelial mucin, is almost completely downregulated upon first full-term pregnancy but is known to be expressed in more than $90 \%$ of invasive ductal carcinomas [2].

A subset of these differentially expressed genes was selected for realtime PCR validation experiments. Small-scale real-time PCR experiments revealed certain trends showing the upregulation of the genes BPAG1, VIM, DCN, RARRES2, EGR1 and ADH1B and the downregulation of $S P A R C$ and $S B E M$ in the mammary gland after pregnancy, but nevertheless individual differences in expression levels were observed. These differences could be due to fluctuations in hormone levels at the time of surgical resection. However, these individual differences also may reflect the differential risk for breast cancer for each of the patients (nulliparous and parous) involved.

Conclusions This study demonstrates that the SAGE technique is useful to detect alterations in gene expression, induced by an early first full-term pregnancy, in the human mammary gland. Our results show that pregnancy induces permanent gene expression modifications in the breast, which can be potentially valuable in the search for predictive markers for breast cancer. Ongoing additional research on these genes in a larger patient population will possibly lead to the development of early markers for breast cancer.

Acknowledgements The authors would like to acknowledge Rob Janssen from the Genome Center of the University of Maastricht for the analysis of the sequencing files. This work is carried out thanks to the financial support of the 'Limburgs Kanker Fonds', the Rotary Club Herne Markvallei and the 'Fonds Slimme Regio' of the Province of Limburg.

References

1. Velculescu VE, Zhang L, Vogelstein B, Kinzler KW: Serial analysis of gene expression. Science 1995, 270:484-487.

2. Miksicek RJ, Myal Y, Watson PH, Walker C, Murphy LC, Leygue $\mathrm{E}$ : Identification of a novel breast- and salivary gland-specific, mucin-like gene strongly expressed in normal and tumor mammary epithelium. Cancer Res 2002, 62:2736-2740. 


\section{P3.02}

The intracellular domain of ErbB4 induces differentiation of mammary epithelial cells RS Muraoka-Cook ${ }^{1,2}$, C Husted'1, D Hunter'1, L Miraglia', HS Earp III1,3,4

${ }^{1}$ UNC Lineberger Comprehensive Cancer Center, ${ }^{2}$ Department of Genetics, ${ }^{3}$ Department of Medicine and ${ }^{4}$ Department of Pharmacology, UNC School of Medicine, Chapel Hill, North Carolina, USA

Breast Cancer Research 2005, 7(Suppl 2):P3.02 (DOI 10.1186/bcr1124) Cell proliferation in the mammary epithelium is stimulated in part by EGF receptor activation, while differentiation requires ErbB4/HER4, prolactin and STAT5A [1]. Unlike other EGFR family members, HER4 undergoes ligand-dependent transmembrane domain cleavage, releasing a soluble 80 $\mathrm{kDa}$ tyrosine kinase ( $\mathrm{s} 80^{\mathrm{HER} 4}$ ) that localizes to the nucleus; the physiologic relevance of $\mathrm{s} 80^{\mathrm{HER}} 4$ is unknown [2]. Using HC11 mouse mammary cells, we showed that EGF, HB-EGF and prolactin increased STAT5A phosphotyrosine and promoter transactivation, but only HBEGF and prolactin induced differentiation markers and organization into polarized three-dimensional, lumen-containing structures in Matrigel. Heregulin did not stimulate lumen formation; rather, it increased and disorganized HC11 cell growth. Selective inhibition of ErbB1/ErbB2 activation unmasked heregulin-dependent differentiation and lumen formation. Kinase-dead HER4, or a HER4V675A mutant abolishing transmembrane cleavage, were expressed in $\mathrm{HC} 11$ cells. HC11 HER4kd or HER4 ${ }^{\mathrm{V} 675 \mathrm{~A}}$ cells exhibited impaired HB-EGF and prolactin-dependent STAT5A translocation, promoter activation and lactogenic marker induction, indicating that both differentiation pathways need ErbB4 kinase activity and s80HER4 formation. $\mathrm{HC} 11$ cells constitutively expressing $\mathrm{S} \mathrm{O}^{\mathrm{HER} 4}$ exhibited basal expression of differentiation markers, increased basal STAT5A activity and three-dimensional lumen formation. These results demonstrate that mammary cell differentiation can be stimulated by HER4 through a process requiring $580^{H E R} 4$ production.

References

1. Long W, Wagner K-U, Lloyd KCK, Binart N, Shillingford JM, Hennighausen L, Jones FE: Impaired differentiation and lactational failure in ErbB4-deficient mammary glands identify ERBB4 as an obligate mediator of Stat5. Development 2003, 130:5257-5268.

2. Carpenter G: ErbB-4: mechanism of action and biology. Exp Cell Res 2003, 284:66-77.

\section{P3.03}

Essential functions of the Janus kinase 2 (Jak2) during mammary gland development and tumorigenesis

\section{K Sakamoto', A Krempler'1, AA Triplett ${ }^{1}$, J Zhu'2, H Rui 2 ,} K-U Wagner ${ }^{1}$

${ }^{1}$ Eppley Institute for Research in Cancer and Allied Diseases, University of Nebraska Medical Center, Omaha, Nebraska, USA; ${ }^{2}$ Department of Oncology, Lombardi Comprehensive Cancer Center, Georgetown University Medical Center, Washington, DC, USA Breast Cancer Research 2005, 7(Suppl 2):P3.03 (DOI 10.1186/bcr1125) Jak2 is a hormone-receptor-coupled kinase that mediates the tyrosine phosphorylation and activation of signal transducers and activators of transcription (Stat). The biological relevance of Jak/Stat signaling in hormone-responsive adult tissues is difficult to investigate since Jak2 deficiency leads to embryonic lethality [1-3]. We therefore generated various Jak2 conditional knockout models to study essential functions of Jak2 during particular stages of mammary gland development and during neoplastic transformation in a Her2/neu-overexpressing breast cancer model. Our experiments show that Jak2 is essential for mammogenesis in virgin, pregnant, and postpartum females [4]. In addition to its pivotal role for mammary epithelial cell proliferation, specification, and differentiation, we demonstrate that this kinase is indispensable for the prolactin-mediated activation of Stat5 and the maintenance of functionally differentiated alveolar cells during lactation. A primary focus of our current research is to examine how Jak/Stat tumorigenesis through overexpression of growth factor receptors, in particular ErbB2 (Her2/neu). The uniqueness of our model design enables us to genetically modify Jak/Stat signaling both prior to growth factor-mediated neoplastic transformation (cancer prevention) and during particular stages of the progressing disease (cancer therapy).

Acknowledgements This work was supported, in part, by the Public Health Service grants CA93797 (to KUW) and CA101841 (to HR and KUW) from the National Cancer Institute. HR receives a Public Health Service grant from the National Institutes of Health (DK052013). AK received a stipend from the Deutsche Forschungsgemeinschaft (DFG, KR 2107/1-1). Support provided to KUW by the Nebraska Cancer and Smoking Disease Research Program (NE DHHS LB595), and the Cattlemen's Ball of Nebraska, Inc., was imperative to finance the generation of the Jak2-deficient animal model.

References

1. Parganas E, Wang D, Stravopodis D, Topham DJ, Marine JC, Teglund S, Vanin EF, Bodner S, Colamonici OR, van Deursen JM, et al:: Jak2 is essential for signaling through a variety of cytokine receptors. Cell 1998, 93:385-395.

2. Neubauer $\mathrm{H}$, Cumano A, Muller M, Wu H, Huffstadt U, Pfeffer K: Jak2 deficiency defines an essential developmental checkpoint in definitive hematopoiesis. Cell 1998, 93:397-409.

3. Krempler A, Qi Y, Triplett AA, Zhu J, Rui H, Wagner KU: Generation of a conditional knockout allele for the Janus kinase 2 (Jak2) gene in mice. Genesis 2004, 40:52-57.

4. Wagner KU, Krempler A, Triplett AA, Qi Y, George NM, Zhu J, Rui $\mathrm{H}$ : Impaired alveologenesis and maintenance of secretory mammary epithelial cells in Jak2 conditional knockout mice. $\mathrm{Mol}$ Cell Biol 2004, 24:5510-5520.

\section{P3.04}

Cooperation between extracellular signaling and intracellular Ras activation leads to immortalization and epithelial-to-mesenchymal transition of variant human mammary epithelial cells

\section{N Dumont, YG Crawford, P Reynolds, TD TIsty}

Department of Pathology and Comprehensive Cancer Center, University of California at San Francisco, California, USA

Breast Cancer Research 2005, 7(Suppl 2):P3.04 (DOI 10.1186/bcr1126) Our laboratory has previously identified a rare subpopulation of variant human mammary epithelial cells (vHMEC) that have the capacity to propagate beyond an in vitro proliferation barrier and accumulate multiple chromosomal changes [1]. These cells contain hypermethylated and silenced p16(INK4a) (p16) promoters and overexpress COX-2. We found evidence that cells with these characteristics exist in disease-free women in morphologically normal tissue [2]. Furthermore, these distinguishing characteristics have also been found in DCIS lesions, indicating that these cells are very relevant to the carcinogenic process. In order to investigate the molecular mechanisms required for these cells to progress to a malignant phenotype, we examined the effect of oncogenic stress on the transformation of vHMEC by introducing constitutively active Ha-Ras V12 into these cells. Consistent with the idea that vHMEC are already engaged in the transformation process, upon exposure to oncogenic stress vHMEC failed to undergo a proliferative arrest as seen in normal fibroblasts or normal epithelium. We have used this model system to examine the early events that control expression of tumorigenic phenotypes in these cells. We find that critical interactions between stromal cells and initiated epithelial cells are necessary for the manifestation of specific tumorigenic phenotypes such as epithelial-to-mesenchymal transition.

\section{References}

1. Romanov SR, Kozakiewicz BK, Holst CR, Stampfer MR, Haupt LM, TIsty TD: Normal human mammary epithelial cells spontaneously escape senescence and acquire genomic changes. Nature 2001, 409:633-637.

2. Holst CR, Nuovo GJ, Esteller M, Chew K, Baylin SB, Herman JG, Tlsty TD: Methylation of $\mathrm{p} 16$ (INK4a) promoters occurs in vivo in histologically normal human mammary epithelia. Cancer Res 2003, 63:1596-1601. 


\section{P3.05}

\section{Ex vivo isolation of adult stem cells from normal and} tumour mouse mammary parenchyma

\section{G Bonizzi, A Cicalese, B Giulini, S Pesce, A Gobbi, S Pece,} PP Di Fiore, PG Pelicci

European Institute of Oncology, Milan, Italy

Breast Cancer Research 2005, 7(Suppl 2):P3.05 (DOI 10.1186/bcr1127)

It is generally believed that tumours originate from adult tissues, in which most cells are quiescent, and that the proliferative advantage of tumour cells arises from their ability to bypass senescence. Once this is obtained, cells can accumulate further genetic alterations that drive progressive transformation into highly malignant derivatives. How normal cells evade the senescence program remains an unresolved issue.

Recent findings suggest the alternative possibility that stem/progenitor cells are the targets of transformation. Many tumours contain only a small subset of cells endowed with the property of uncontrolled growth (cancer stem cells). Although the program of senescence in stem cells is suppressed, their lifespan is restrained by signalling pathways ( $p 19$ p53; p16-Rb) that are activated by DNA damage (telomere dysfunction, environmental stresses). It is proposed that inactivation of these pathways in stem cells might contribute to tumour formation.

We set out to cultivate in vitro breast stem cells from the mouse mammary gland (BSC) based on their ability to survive in suspension as 'mammospheres' and to differentiate into myoepithelial and epithelial cells. Murine mammospheres do not result from passive cell aggregation and have clonal origin, as assessed by labelling cell membranes with different epifluorescent dyes. To prove the 'staminality' of these cellular populations we performed in vivo reconstitution experiments, by inoculating single cell suspensions of mammospheres in the mouse cleared fat pad.

Using the same experimental approach, we were also able to generate mammospheres from MMTV-ErbB2(cNeu) transgenic mice. These mice develop polyclonal mammary adenocarcinomas arising synchronously. The mammospheres arising from MMTV-ErbB2 mice show a dramatically prolonged lifespan upon serial passages and are bigger than their normal counterparts (more than 1000 cells/sphere vs 200-400 cells in the w.t. mice). To prove that cancer mammospheres are enriched in cancer BSC, we are performing transplantation experiments in which single cell suspensions of these mammospheres are inoculated in the normal gland of singenic mice.

\section{P3.06}

\section{Myoepithelial cell layer disruption and human breast cancer invasion YG Man', QX Sang ${ }^{2}$}

${ }^{1}$ Department of Gynecologic and Breast Pathology, Armed Forces Institute of Pathology and American Registry of Pathology, Washington, DC, USA; ${ }^{2}$ Department of Chemistry and Biochemistry, and Institute of Molecular Biophysics, Florida State University, Tallahassee, Florida, USA

Breast Cancer Research 2005, 7(Suppl 2):P3.06 (DOI 10.1186/bcr1128) Background Human breast luminal epithelium and the stromal compartment are separated by a layer of myoepithelial (ME) cells and basement membrane, whose disruption is required for tumor invasion. Basement membrane degradation has been ascribed largely to an overproduction of proteases by the tumor and stromal cells. However, the causes and phenotypes of ME cell layer disruption and its contribution to the initiation of tumor invasion remain to be further explored.

Methods Human ductal carcinoma in situ samples with ME disruption and microinvasion were sectioned and immunohistochemically stained with Ki-67, estrogen receptor (ER), leukocyte common antigen, and other biochemical markers.
Results A subset of pre-invasive and micro-invasive tumors contained focal disruptions in their ME cell layer. ER-negative cell clusters overlying a focally disrupted ME cell layer and the basement membrane showed a significantly higher proliferation rate than adjacent cells within the same duct. These disruptions were associated with histochemical and genetic alterations in the overlying tumor cells, including the loss of ER expression, a higher frequency of loss of heterozygosity, and a higher expression of cell cycle, angiogenesis, and invasion-related genes. Focal ME layer disruptions were, in general, associated with a higher rate of epithelial proliferation and leukocyte infiltration; however, a small fraction of these ER-negative cells lacked proliferation and differentiation markers resembling dormant cancer stem cells.

Conclusions We propose a novel hypothesis that a localized death of ME cells and immunoreactions that accompany an external environmental insult or internal genetic alterations are triggering factors for ME layer disruptions, basement membrane degradation, and subsequent tumor progression and invasion. Inflammation may contribute to the death of focal ME cells. Putative dormant cancer stem cells may be partially responsible for tumor drug resistance and recurrence.

Acknowledgements Supported in part by Congressionally Directed Medical Research Program/DOD grants DAMD17-01-1-0129 and DAMD17-01-1-0130 to YGM, and by DAMD17-02-1-0238, NIH grant CA78646, and Florida State University grants to QXS.

\section{References}

1. Man YG, Sang QX: The significance of focal myoepithelial cell layer disruptions in human breast tumor invasion: a paradigm shift from the 'protease-centered' hypothesis [review]. Exp Cell Res 2004, 301:103-118.

2. Man YG, Shen T, Sang OX, Berg PE, Schwartz AM: Cell clusters in a subset of in situ breast tumors show an unusual growth pattern: implications for invasion and metastasis. Cancer Detect Prevent 2005, in press.

\section{P3.07}

Imprint as a reliable diagnostic tool in breast cancer and possible usefulness for research purposes E Mortensen', L Hansen', JO Frantzen², S Klenow 2 , N Bjurstam ${ }^{2}$, RH Paulssen ${ }^{3}$

${ }^{1}$ Department of Pathology, ${ }^{2}$ Department of Radiology and ${ }^{3}$ Institute of Clinical Medicine, University Hospital, North Norway, Tromso, Norway Breast Cancer Research 2005, 7(Suppl 2):P3.07 (DOI 10.1186/bcr1129) Objective The aim in breast cancer treatment is to provide the correct diagnosis with minimal delay that makes it possible to immediately plan further treatment with the patient. In addition, the imprint method can be used to make a diagnosis on material that will be snap-frozen for future research purposes.

Method Imprints are made by gently pushing the core biopsy to a coated glass slide, and then air-drying and staining with Diff-Quick. The diagnosis is usually made within $5 \mathrm{~min}$.

Results Of 51 imprints, 15 were diagnosed as carcinoma. Histology confirmed carcinoma in 13. The two apparent false positives turned out to be cancer on further investigation. Two of the imprints were given a benign diagnosis; both turned out to be invasive lobular carcinoma. The rest of the imprints that were given a benign diagnosis were all confirmed as benign on histology.

Conclusions There was no true false positive diagnosis, but there were two false negatives, both invasive lobular carcinoma. Imprint of core biopsies is a reliable cytological method for diagnosing invasive ductal carcinoma in breast. The diagnosis is reached within minutes and treatment can be planned without delay, which ensures optimal patient care. In addition, this method can be used to establish a diagnosis on material that will be snap-frozen for future research purposes. 


\section{P3.08}

Immortalization-associated gene signature in breast cancer

SH Dairkee', M Nicolau², S Champion'1, Y Ji², A Sayeed', Z Meng', J Demeter ${ }^{3}$, SS Jeffrey ${ }^{2}$

${ }^{1}$ California Pacific Medical Center Research Institute, San Francisco, California, USA; ${ }^{2}$ Department of Surgery, Stanford University School of Medicine, Stanford, California, USA; ${ }^{3}$ Department of Genetics, Stanford University School of Medicine, Stanford, California, USA Breast Cancer Research 2005, 7(Suppl 2):P3.08 (DOI 10.1186/bcr1130)

Background Cell immortalization has been considered a hallmark of malignancy, yet the molecular signature identifying a tumor's ability to immortalize is not known. To better understand the immortalization process, we used in vitro model systems developed from clinical primary breast cancer specimens. While spontaneous immortalization of tumor cells in vitro is a rare event, immortalization can be achieved through the introduction of the hTERT component of telomerase. Because spontaneously arising cancer cell lines are already immortal and display high levels of telomerase expression, they are not amenable for study of the immortalization process in malignancy. However, finite life primary tumor cultures show low $h T E R T$ expression, providing a tool to study the effect of exogenously introduced $h T E R T$.

Methods and results We introduced the $h T E R T$ gene into 17 primary breast cancer cultures and analyzed global gene expression changes before and after hTERT transduction in nine cases. All transduced cultures achieved immortalization without other genetic manipulation. Using 42,000 feature cDNA microarrays, we identified 594 genes (the immortalization-associated signature [IAS]) that distinguished $h T E R T$ transduced and non-transduced cultures. We suggest that hTERT immortalization reflects a physiologic process since expression patterns of $h T E R T$-transduced and spontaneously immortalized primary cultures along IAS genes were correlated. To identify whether these genes relate to in vivo breast cancer, we used the IAS to supervise the analysis of 295 breast cancer patients. The signature strongly predicted distant metastasis-free $(P=0)$ and overall survival $(P<0.001)$ in ER-positive tumors but not in ER-negative tumors. Notably, hTERT gene expression level itself did not predict clinical outcome in primary tumors.

Conclusion To our knowledge, this is the first demonstration of an immortalization signature in human breast cancer. This signature is strongly associated with specific cellular pathways in human tumors that may underlie the immortalization process and influence tumor behavior. The association between the IAP signature and outcome for ER-positive tumors but not ER-negative tumors suggests that different pathways are involved in the outcome of ER-negative tumors.

\section{P3.09}

\section{Morphogenesis of the mammary gland and the role} of keratin expression

\section{A Aldahmash ${ }^{1,2}$, D Lunny $y^{1,3}$, B Lane $e^{1,3}$}

${ }^{1}$ CRUK Cell Structure Research Group, School of Life Sciences, University of Dundee, UK; ${ }^{2}$ Anatomy Department, College of Medicine, King Saud University, Riyadh, Kingdom of Saudi Arabia; ${ }^{3}$ Centre for Molecular Medicine, Singapore

Breast Cancer Research 2005, 7(Suppl 2):P3.09 (DOI 10.1186/bcr1204) The selective expression of keratin genes is highly tissue-specific, and antibodies to keratins have long been used as markers of differentiation in cell and developmental biology and in pathology applications. Antibodies to keratins can mark the progress of normal versus abnormal differentiation, can detect early apoptotic changes and may even identify stem cell-enriched tissue populations. A better understanding of the function of keratins has come from identifying links between keratin mutations and a wide range of tissue fragility disorders, which have shown that keratin intermediate filament proteins contribute physical resilience to epithelial tissues. The tissue specificity of keratins may thus reflect different requirements for stiffer or more development of the mammary gland to ask whether differences in the plasticity of cell compartments, proposed to result from expression of different keratins, may contribute to morphogenesis in development. If so, such physical differences might also be important in cancer metastasis. During early development, mouse mammary glands, like other epidermal appendages, first appear as thickenings of the ectoderm, which then expand to form solid buds of epithelial cells protruding into the mesenchyme. These buds then undergo substantial growth and remodeling. Many well-established signaling molecules are now known to play a role in epidermal appendage development, yet the physical changes that take place within the cells at these early stages, and which ultimately enable them to develop their functional tissue morphology, are still poorly understood. Using immunohistochemistry on mouse mammary gland precursors between days 12 and 19 of gestation, we mapped the sequential changes in expression of keratins and some other key structural proteins. Compartmentalization of keratin expression was observed to divide the mammary gland rudiment into a number of different zones. The subsequent fate of these zones suggests that the specific expression of keratins and other structural proteins may indeed predict the functional capacity of cell populations in tissues.

\section{P4.01}

\section{6-(1-oxobutyl)-5,8-dimethoxy-1,4-naphthoquinone} exerts anti-angiogenic activity via inhibition of vascular endothelial cell growth factor and hypoxiainducible factor 1 alpha in hypoxia-exposed MCF breast cancer cells

\section{HJ Lee, SH Kim}

Department of Oncology, Graduate School of East-West Medical Science, Kyunghee University, Yongin, South Korea

Breast Cancer Research 2005, 7(Suppl 2):P4.01 (DOI 10.1186/bcr1131)

Background Hypoxia induces the transcription of various genes involved in angiogenesis and anaerobic metabolism necessary for the growth of tumor cells. Hypoxia-inducible factor 1 alpha (HIF-1 $\alpha$ ) regulates genes involved in the response to hypoxia and promotes neoangiogenesis in cancer. Thus, to develop an anticancer agent with antiangiogenic activity in hypoxic cancer cells, 6-(1-oxobutyl)-5,8-dimetoxy1,4-naphthoquinone $(\mathrm{OXO})$ was synthesized.

Methods The XTT (2,3-bis[2-methoxy-4-nitro-5-sulfophenyl]-2H-tetrazolium-5-carboxanilide) assay for cytotoxicity, the ELISA, RT-PCR and western blotting analysis were employed in MCF-7 human breast cancer cells under hypoxic conditions.

Results OXO showed cytotoxicity against MCF-7 cells, human breast cancer cells with an $\mathrm{IC}_{50}$ value of $10 \mu \mathrm{M}$. OXO also reduced the levels of vascular endothelial cell growth factor (VEGF) and HIF- $1 \alpha$ in MCF cells exposed to hypoxia. Similarly, OXO downregulated the expression of HIF- 1 and VEGF by western blotting and RT-PCR. In addition, OXO

\section{Figure 1}

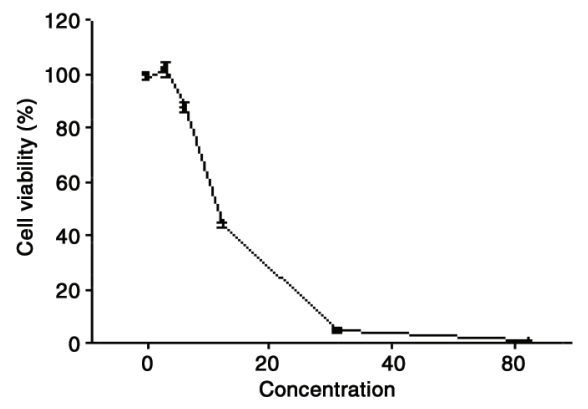

Cytotoxicity. 
Figure 2

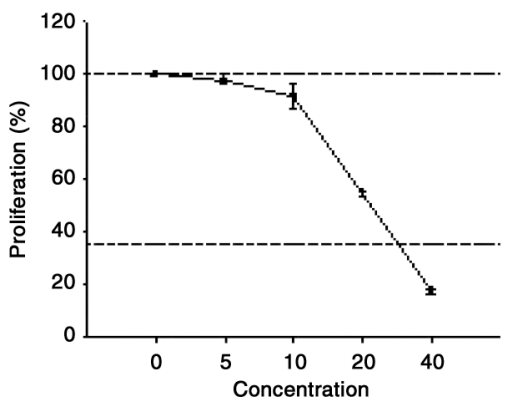

Effect of OXO on the proliferation of bFGF-treated HUVECs.

\section{Figure 3}
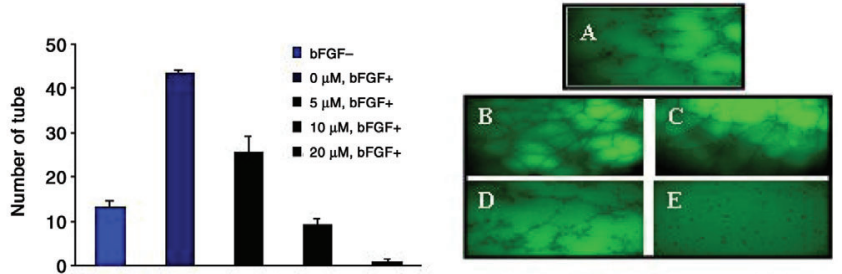

Effect of OXO on the tube formation of bFGF-treated HUVECs.

\section{Figure 4}

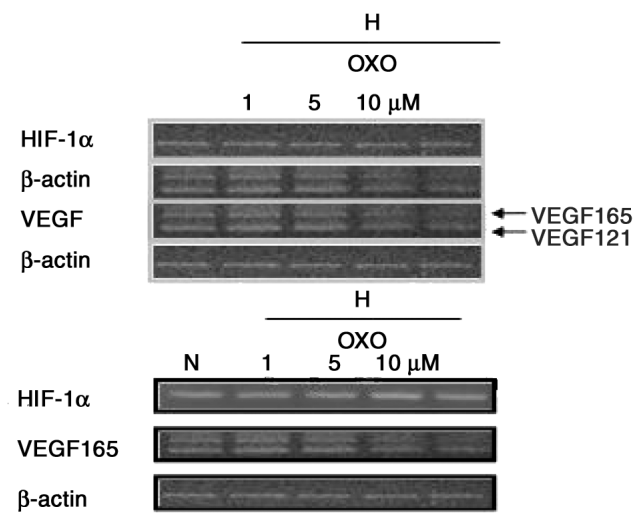

Effect of OXO on VEGF and HIF-1 $\alpha$ in MCF-7 cells exposed to hypoxia.

inhibited the basic fibroblast growth factor (bFGF)-induced proliferation, inhibited tube formation of human umbilical vein endothelial cells (HUVECs) and also disrupted the neovascularization in bFGF-treated Matrigel in vivo.

Conclusion Taken together, these results show that OXO may exert anti-tumor and anti-angiogenic activity against MCF-7 cells via regulation of HIF- $1 \alpha$ and VEGF.

References

1. Song G-Y, Kim Y, You YJ, Cho H, Kim SH, Sok DE, Ahn BZ: Naphtazarin derivatives (VI): synthesis, inhibitory effect on DNA topoisomerase-I and antiproliferative activity of 2-or 6(1-oxyiminoalkyl)-5,8-dimethoxy-1,4-napthoquinones. Arch Pharm Pharm Med Chem 2000, 333:87-92.
Figure 5

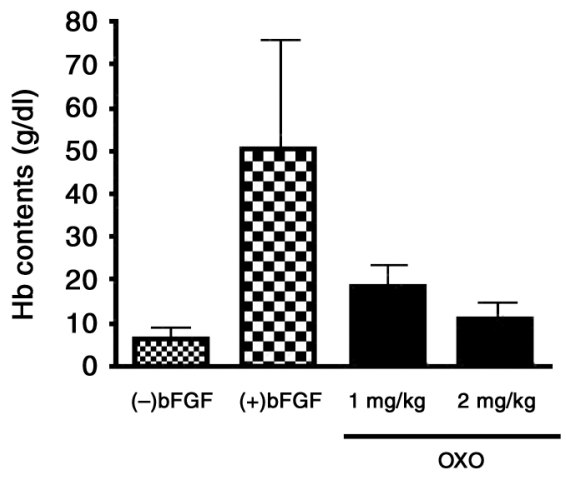

Effect of OXO on the content of hemoglobin in bFGF-treated Matrigel.

2. Forsythe JA, Jiang BH, Lyer NV, Agani F, Leung SW, Koos RD, et al.: Activation of vascular endothelial growth factor gene transcription by hypoxia-inducible factor 1. Mol Cell Biol 1996, 16: 4604-4613.

3. Richard DE, Berra E, Pouyssegur J: Angiogenesis: how a tumor adapts to hypoxia. Biochem Biophys Res Commun 1999, 266: 718-722.

4. Ferrara $\mathrm{H}$, Gerber H-P, Lecouter J: The biology of VEGF and its receptors. Nat Med 2003, 9:669-676.

\section{P4.02}

\section{HER2 upregulates fatty acid synthase and acetyl-CoA} carboxylase at a translational level in breast cancer

\section{cells}

S Yoon 1,2, M-Y Lee 1,2, B-W Park ${ }^{3}$, K-S Kim 1,2

${ }^{1}$ Department of Biochemistry and Molecular Biology, ${ }^{2}$ Center for Chronic Metabolic Disease Research and ${ }^{3}$ Department of Surgery, BK21 Project, Yonsei University, College of Medicine, Seoul, Korea Breast Cancer Research 2005, 7(Suppl 2):P4.02 (DOI 10.1186/bcr1132) Overexpression of the HER2 oncogene is observed in approximately $30 \%$ of human breast carcinoma specimens. HER2-overexpressing breast cancer cells, such as SK-BR-3 and BT-474 cells, express fatty acid synthase (FAS) at a higher level than MCF-7 cells or MDA-MB-231 cells, where HER2 is expressed at a moderate or low level. Adenovirusmediated HER2 expression in MDA-MB-231 cells increased acetyl-CoA carboxylase alpha $(A C C \alpha)$ and FAS protein levels without significant increases of their mRNA. HER2-mediated increases of ACC $\alpha$ and FAS proteins were inhibited by the PI3K inhibitor, LY294002, and the mTOR inhibitor, rapamycin. But sterol regulatory element-binding protein 1 (SREBP1) and ATP citrate lyase (ACL) mRNA and protein levels were not changed by HER2 overexpression, LY294002 or rapamycin. In conclusion, our results suggest that HER2-overexpressing cells lead to increased ACC $\alpha$ and FAS proteins at a translational level via the activation of the mTOR signaling pathway and not through SREBP-1cmediated transcriptional activation.

\section{P4.03}

\section{Altered signaling in anti-estrogen-resistant human} breast cancer cells AE Lykkesfeldt, T Frogne, JS Jepsen, CK Fog, SS Larsen Department of Tumor Endocrinology, Institute of Cancer Biology, Danish Cancer Society, Copenhagen, Denmark

Breast Cancer Research 2005, 7(Suppl 2):P4.03 (DOI 10.1186/bcr1133) Breast cancer patients with advanced disease often benefit from endocrine therapy. However, many patients develop acquired resistance to treatment after a period of response. In the Department of 
Tumor Endocrinology we have established several human breast cancer cell lines with acquired anti-estrogen resistance through longterm treatment with different anti-estrogens. These cell lines have been used for our studies of the signaling pathways, which may be activated in cells with acquired anti-estrogen resistance.

Analysis of the expression of genes known to be important for human breast cancer has revealed that the majority of the anti-estrogenresistant breast cancer cell lines have decreased estrogen receptor expression and signaling. However, increased expression of phosphorylated PKB/Akt (p-Akt) and Akt kinase activity was observed in several anti-estrogen-resistant cell lines. The PI3 kinase is an upstream signaling molecule for Akt, and inhibition of PI3-kinase activity with wortmannin or LY294002 deceases the level of p-Akt. Both PI3kinase inhibitors inhibited growth of the resistant cells. However, wortmannin displayed a more profound growth inhibitory effect on antiestrogen-resistant cell lines than on parental MCF-7 cells. Treatment with the novel Akt inhibitor $\mathrm{SH}-6$ resulted in a very strong growth inhibition of three resistant cell lines overexpressing p-Akt, whereas the parental MCF-7 cells were significantly less growth inhibited.

It was investigated whether the increased level of p-Akt in the resistant cells was due to signaling from IGF-IR and IRS-1, or whether it resulted from decreased PTEN activity. Both involvement of IGF-IR and PTEN could be excluded.

At present, our working hypothesis is that anti-estrogen-resistant human breast cancer cell lines with an increased p-Akt level require signaling via activated Akt to survive and maintain growth in the presence of the anti-estrogen. Studies on clinical material will be important to evaluate whether anti-estrogen-resistant tumors overexpress p-Akt and whether Akt may be a target for treatment of anti-estrogen-resistant breast cancer.

\section{P4.04}

\section{HIN-1, an inhibitor of cell growth, invasion, and AKT1 activation \\ IE Krop, MT Parker, N Qimron, D Porter, K Polyak}

Department of Medical Oncology, Dana-Farber Cancer Institute and Department of Medicine, Harvard Medical School, Boston, Massachusetts, USA

Breast Cancer Research 2005, 7(Suppl 2):P4.04 (DOI 10.1186/bcr1134)

Background High in normal $1(\mathrm{HIN}-1)$ is a small, secreted protein that was initially identified as a protein the expression of which is lost in the vast majority of breast cancers. The silencing of $\mathrm{HIN}-1$ expression is due to methylation of its promoter, which in addition to breast cancer also occurs in a significant fraction of many other types of solid tumors including prostate cancer, lung cancer, pancreas cancer, and retinoblastoma, suggesting a potential tumor suppressor function. Consistent with this hypothesis, in non-small-cell lung cancer, downregulation of $\mathrm{HIN}-1$ expression was found to be the most significant independent predictor of poor clinical outcome in stage I disease, suggesting loss of $\mathrm{HIN}-1$ expression is a functionally important event. The receptor of $\mathrm{HIN}-1$ is unknown, but ligand-binding studies indicate the presence of high-affinity cell surface $\mathrm{HIN}-1$ binding sites on the same epithelial cells that express $\mathrm{HIN}-1$, suggesting that $\mathrm{HIN}-1$ functions as an autocrine factor.

Methods To further explore the function and mechanism of action of $\mathrm{HIN}-1$, we examined the effect of HIN-1 on immortalized mammary epithelial cells and a panel of breast cancer cell lines in vitro. $\mathrm{HIN}-1$ was delivered via multiple expression systems, including a tetracyclineregulated expression system, recombinant adenovirus, and recombinant $\mathrm{HIN}-1$ fusion protein.

Results We find that HIN-1 is a potent inhibitor of anchoragedependent and anchorage-independent cell growth. Expression of HIN-1 in MDA-MB-435 cells causes reversion of their invasive morphology when these cells are grown in a three-dimensional culture system. As further evidence that $\mathrm{HIN}-1$ can influence invasive behavior, expression of HIN-1 markedly inhibited the invasion and migration of breast cancer cell lines in Boyden chamber assays. Expression of phosphorylation of the retinoblastoma protein $(\mathrm{Rb})$, while in exponentially growing cells HIN-1 induces apoptosis without apparent cell cycle arrest or an effect on $\mathrm{Rb}$ phosphorylation. To begin to dissect the mechanism by which HIN-1 suppresses growth, we analyzed the activation status of various signal transduction pathways involved in cell proliferation and survival using activation state specific antibodies. This investigation revealed that mitogen-induced phosphorylation of Akt (Ser 473) is inhibited in $\mathrm{HIN}-1$-expressing cells. Expression of $\mathrm{HIN}-1$ also inhibits Akt-mediated retention of p27 in the cytoplasm. Further supporting the role of Akt in HIN-1-mediated growth inhibition, expression of constitutively activated Akt abrogates HIN-1-mediated growth arrest.

Conclusion Taken together, these studies provide further evidence that $\mathrm{HIN}-1$ possesses tumor suppressor functions and suggest that these activities may be mediated through the Akt signaling pathway.

\section{P4.05 \\ Expression of STAT1 target genes and interferon gamma in human mammary carcinoma tissue W Doppler', C Marth'2, G Daxenbichler' ${ }^{2}$, P Obrist ${ }^{3}$, C Berlato'}

${ }^{1}$ Division Medical Biochemistry, Biocenter, ${ }^{2}$ Department of Gynecology and ${ }^{3}$ Institute of Pathology, Innsbruck Medical University, Innsbruck, Austria

Breast Cancer Research 2005, 7(Suppl 2):P4.05 (DOI 10.1186/bcr1135) Background The signal transducer and activator of transcription 1 (STAT1) in human primary mammary carcinoma was found to be a predictor of good prognosis for the outcome of disease [1]. This is in accordance with its documented role in growth arrest and in proapoptotic signaling.

Methods In order to define signaling pathways employed by STAT1 to exert its effect on the tumor and to define the role of interferon gamma (IFN- $\gamma$ ) in its activation, we have investigated the expression of known STAT1 target genes and of IFN- $\gamma$ in the primary tumor by quantitative RT-PCR. The study was performed with a total of 111 different primary tumor samples.

Results The expression of the two tumor suppressor genes IRF-1 and suppressor of cytokine signaling 1 (SOCS1) were found to be correlated with the activation status of STAT1, as determined by measuring tyrosine phosphorylation of STAT1 by western blotting, DNA binding by electromobility shift assays and nuclear localization by immunohistochemistry. IFN- $\gamma$ expression was correlated to the expression of some, but not all, STAT1 target genes. However, it did not correlate with constitutive STAT1 activation. Survival analysis revealed that, in contrast to STAT1 activation, IFN- $\gamma$ expression was not a predictor of a longer overall or relapse-free survival.

Conclusions Our results indicate that, in the majority of primary mammary carcinomas investigated, the constitutive activation of STAT1 does not depend on increased IFN- $\gamma$ secretion (e.g. as a result of an inflammatory reaction in the tumor). This suggests a prominent role for IFN- $\gamma$-independent mechanisms leading to the constitutive activation of STAT1 in primary mammary carcinomas. The frequent induction of the tumor suppressor genes SOCS1 and IRF-1 in carcinoma tissue with activated STAT1 implies a potential role of these genes in mediating the good prognostic effects of STAT1 activation.

Acknowledgement Supported by the Austrian National Bank, Project No. 10263.

Reference

1. Widschwendter A, Tonko-Geymayer S, Welte T, Daxenbichler G, Marth C, Doppler W: Prognostic significance of signal transducer and activator of transcription 1 activation in breast cancer. Clin Cancer Res 2002, 8:3065-3074. 


\section{P4.06}

\section{In vitro models for tumor protein d52 function in} cancer cells

\section{Shehata ${ }^{1,2}$, R Boutros ${ }^{1,2}$, RK Bright ${ }^{3}$, J Byrne ${ }^{1,2}$}

${ }^{1}$ Molecular Oncology Laboratory, Oncology Research Unit, The Children's Hospital at Westmead, Australia; 'University of Sydney, Discipline of Paediatrics and Child Health, The Children's Hospital at Westmead, Australia; ' ${ }^{3}$ Department of Microbiology and Immunology, Texas Tech University Health Sciences Center, Lubbock, Texas, USA Breast Cancer Research 2005, 7(Suppl 2):P4.06 (DOI 10.1186/bcr1136) Background Tumor protein D52 (TPD52), a tumor-associated antigen, is overexpressed in the majority of breast, prostate and ovarian cancers, where it is also amplified in a proportion of cases. Comparing TPD52 (or D52) protein expression with clinical parameters indicates that increased D52 expression is an early event in the development of prostate cancer and possibly other cancers. The D52 protein is a member of a family that includes the related proteins D53 and D54. Studies to date indicate that while D52-like proteins share common molecular functions as putative adaptor proteins, D52-like genes are not equally overexpressed or targeted by gene amplification in cancer. As a first step in allowing the specific targeting of D52 overexpression in cancer, it is therefore imperative to determine the effects of increasing or reducing the expression of D52 and related proteins in mammalian cells.

Methods Expression vectors encoding human D52, two human isoforms of both D53 and D54, and mouse D52 have been constructed in the pCDNA3.1 vector. Similarly, expression constructs have been derived that encode pEGFP-tagged forms of human D52, and two human D53 isoforms. Transient and stable DNA transfections were carried out using Lipofectamine 2000 reagent into the MDA-MB231 human breast carcinoma cell line, and the Balb/c 3ТЗ fibroblastic cell line. In addition, an Ambion pSilencer system is being developed to reduce D52 expression in MCF-7 breast carcinoma cells. Protein expression in transfected cell populations is assessed using western blotting and indirect immunofluorescence. Cell proliferation rates are assessed using MTT assays, and anchorage-independent growth is assessed by quantitating colony formation in soft agar after a 2-week assay period.

Results We have previously studied the effects of expressing D52 or D53 in MDA-MB-231 breast carcinoma cells, which express relatively low levels of both proteins. While stably transfected MDA-MB-231 cell lines could be derived when D52 or D53 were expressed from the $\beta$ actin promoter, these could not be obtained when pEGFP-tagged D52 and D53 or untagged D53 were expressed from the CMV promoter. Transient transfections revealed that expression of these proteins commonly produced high proportions of multinucleated cells $(40-44 \%$ by 24 hours post transfection), compared with vector controls. Expressing mouse D52 under the control of the CMV promoter increased the cellular proliferation rate in pooled stable 3T3 transfectants, relative to the vector alone, as measured using MTT assays. We are currently carrying out similar transfections with additional D52-like expression constructs in 3T3 cells, and knocking down D52 expression in MCF7 breast carcinoma cells, which express high levels of D52 and D53 proteins.

Conclusions These studies indicate that the exogenous expression of D52-like proteins produces different phenotypes in different cell types. Expressing multiple D52-like proteins in MDA-MB-231 cells adversely affects their ability to complete mitosis, whereas increased proliferation rates in mouse D52-expressing 3T3 cells support previous results implicating D52 as a regulator of cell proliferation in human leukemic and chick neuroretinal cells. Further studies are now required to determine whether reducing D52 expression negatively impacts upon the growth of breast cancer cells.

\section{P4.07}

Functional characterization of genes involved in the development of breast cancer

E Gjernes ${ }^{1,2,3}$, GL Størvold 1,2,3, H Askautrud 1,3, A-L Børresen-Dale ${ }^{1}$, CM Perou ${ }^{2}$, E Frengen ${ }^{1,2,3}$

${ }^{1}$ The Norwegian Radium Hospital, Oslo, Norway; ${ }^{2}$ University of North Carolina, Lineberger Comprehensive Cancer Center, Chapel Hill, North Carolina, USA; ${ }^{3}$ Institute of Medical Genetics, Ullevål University Hospital, Oslo, Norway

Breast Cancer Research 2005, 7(Suppl 2):P4.07 (DOI 10.1186/bcr1137) Amplifications of large chromosomal regions have been detected by microarray-based comparative genomic hybridization in breast tumors and breast cancer cell lines. Many of the genes within these amplicons also show increased expression on microarrays, indicating that these genes are probably of importance in breast cancer development. A significant number of the genes showing both amplifications and increased expression are located in amplified regions of human chromosome 8q. Copy number abnormalities on chromosome $8 q$ are further correlated with poor survival outcome and the presence of TP53 mutations. In our research we used cell-line-based functional assays to identify and characterize genes on human chromosome $8 q$ to identify those that directly contribute to the evolution of breast tumors. Hierarchical clustering of tumor gene expression profiles has identified distinct subtypes of breast tumors. We have chosen specific breast cancer cell lines in our studies as models for two of these subtypes: basal-like and luminal breast tumors. In order to knock down gene expression in these cell lines, we constructed a self-inactivating murine stem cell virus (MSCV)-based shRNA expression vector. The vector, pSiRPG, contains a highly efficient selection marker (Puromycin) and an Enhanced Green Fluorescent Protein marker for the detection of cells harboring the constructs. We have demonstrated efficient expression of siRNAs in cell lines that are difficult to transfect using standard methods. We also constructed a Gateway-compatible expression vector, pRetroTRexD30, on the MSCV back bone. Fulllength cDNAs from more than 30 chromosome $8 q$ genes selected in our project have so far been transferred into this vector.

The siRNA constructs are currently being used to knock down gene expression in cell lines showing high endogenous expression of these $8 q$ genes. In parallel experiments, the genes are overexpressed in cell lines showing low or undetectable endogenous expression. In these experiments, cell lines harboring overexpression or siRNA constructs are assayed for cancer-relevant phenotypes such as growth rate changes, the ability to grow in low serum concentrations and/or hypoxic conditions, sensitivity to chemotherapeutic drugs, apoptosis induction, and the ability to form colonies in agar.

Acknowledgements This work is supported by the Research Council of Norway and the Norwegian Cancer Society, and by funds from the $\mathrm{NCl}$ Breast SPORE program to CMP (P50-CA58223-09A1).

\section{P4.08}

Abstract withdrawn. 


\section{P4.09 \\ Deletions at the chromosome 3 common eliminated region 1 on 3p21.3 in human breast tumors ThE Petursdottir ${ }^{1}, \mathrm{U}$ Thorsteinsdottir ${ }^{2}$, JG Jonasson ${ }^{1,3}$,

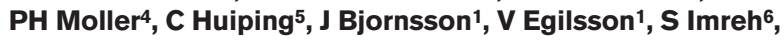 S Ingvarsson 5}

${ }^{1}$ Department of Pathology, Landspitali University Hospital of Iceland, Reykjavik, Iceland; ${ }^{2}$ DeCode Genetics, Reykjavik, Iceland; ${ }^{3}$ Icelandic Cancer Registry, The Icelandic Cancer Society, Reykjavik, Iceland; ${ }^{4}$ Department of Surgery, Landspitali University Hospital of Iceland, Reykjavik, Iceland; 5 Institute for Experimental Pathology, University of Iceland, Reykjavik, Iceland; ${ }^{\circ}$ Microbiology and Tumor Biology Center, Karolinska Institute, Stockholm, Sweden

Breast Cancer Research 2005, 7(Suppl 2):P4.09 (DOI 10.1186/bcr1139) Background Chromosome 3 is abnormal in a variety of human cancers. An assay, based on a non-random elimination of human chromosome segments in mouse-human microcell hybrids during tumor growth in SCID mice, has been developed. It is called the Elimination test $(\mathrm{Et})$, and is designed for the identification of chromosomal regions containing putative TSGs. A commonly lost segment, termed chromosome 3 common eliminated region 1 (C3CER1) (also referred to as CER1), was identified using the Et. This region spans $\sim 2.4 \mathrm{Mb}$ at $3 p 21.3$ and contains 33 active genes, including the putative TSGs: $L F, L I M D 1$ and $R / S 1$. It was recently reported that the LIMD1 gene product functions in connection with pRB to suppress cell proliferation. Here, we addressed the question of whether the C3CER1 region at $3 p 21.3$ is preferentially lost in actual human breast tumors, and whether the fragile nature of FRA3B induces terminal deletions leading to 3p14.2-pter losses or whether the eventual 3p21.3 losses are interstitial. We also analysed whether the LIMD1 gene was mutated in human breast tumors.

Methods To evaluate the loss of C3CER1 in human tumor tissues we performed loss of heterozygosity $(\mathrm{LOH})$ analysis of 159 breast tumors. We compared the deletion frequency of the C3CER 1 area with two other regions on 3p; that is, the FHIT/FRA3B region at 3p14.2 and VHL at 3p25.3. The tumor material was screened for mutations with the SSCP method, and samples with abnormal mobility in SSCP gels were sequenced in an $\mathrm{ABI} 3100$ genetic analyser. We are conducting multipoint $\mathrm{FISH}$ analysis to confirm microdeletions in the breast tumor specimens. Results LOH was detected in the C3CER1 region in $84 \%$ of informative tumors. Thirty-nine percent of $\mathrm{LOH}$-positive tumors showed $\mathrm{LOH}$ at all informative C3CER1 markers. The other $61 \%$ had a discontinuous $\mathrm{LOH}$ pattern suggesting interstitial deletions or breakpoints. In the VHL and FHIT regions, deletions were observed in $69 \%$ and $30 \%$ of tumors, respectively. We found polymorphism in the first three exons of LIMD1, but sequencing revealed no mutations leading to changes in the protein product.

Conclusion Of the three $3 p$ regions analysed, the highest deletion frequency was observed at the C3CER1 region. We demonstrate that the interstitial deletions including C3CER1 prevail over 3p14.2-pter losses. The LIMD1 gene is not frequently mutated in breast cancer biopsies.

Acknowledgements This research was funded by The Science fund of Landspitali University Hospital, The Science fund of the Icelandic Cancer Society, and The Memorial fund of Bergthora Magnusdottir and Jakob B. Bjarnason.

\section{P4.10}

\section{Protein expression and gene amplification of primary} cyclins ( $A, B_{1}, D_{1}, D_{3}$ and $\left.E\right)$ and secondary cyclins ( $C$ and $H)$ in relation to prognosis in breast cancer patients A Husdal1,2, G Bukholm,2, IRK Bukholm²,3

${ }^{1}$ Institute of Clinical Epidemiology and Molecular Biology, Akershus University Hospital, Nordbyhagen, Norway; ${ }^{2}$ University of Oslo, Norway; ${ }^{3}$ Department of Surgery, Akershus University Hospital, Nordbyhagen, Norway

Breast Cancer Research 2005, 7(Suppl 2):P4.10 (DOI 10.1186/bcr1140) Deregulation of cell cycle control is a hallmark of cancer. The primary cyclins $\left(A, B_{1}, D_{1}, D_{3}\right.$ and $\left.E\right)$ are crucial for cell cycle progression [1]. propulsion and have not been previously evaluated in breast cancer. We have examined protein expression and gene amplification of cyclins in breast carcinomas and correlated the findings with clinical follow-up data. We have previously demonstrated that overexpression of cyclin $A$ is associated with poor prognosis in breast cancer patients [2]. In this study we wanted to evaluate the impact of other cyclins, both at the gene level and at the protein level. We wanted to evaluate whether the overexpression of cyclins is a result of gene amplification, as well as to evaluate the prognostic value of gene amplification of different cyclins for breast cancer patients. The impact of TP53 gene mutations on gene amplification of cyclins was also evaluated.

Real-time quantitative PCR was used to detect gene amplification of cyclin $A$, cyclin $B_{1}$, cyclin $C$, cyclin $D_{1}$, cyclin $D_{3}$, cyclin $E$ and cyclin $H$ in tumour tissue from 80 patients operated for invasive breast carcinomas, while immunohistochemistry was applied to detect protein expression of the same cyclins.

Among the 80 breast cancer tumour samples examined, $26.7 \%$ was defined to have $c c n A_{2}$ gene amplification, 37.2\% had $c c n B_{1}$ gene amplification, $82.6 \%$ of the samples harboured amplification of $c c n C$, $74.4 \%$ had $c c n D_{1}$ gene amplification, $41.9 \%$ had $c c n D 3$ gene amplification, $29.1 \%$ of the patients had ccnE gene amplification and $9.3 \%$ of the samples showed amplification of the ccnH gene.

When correlation between gene amplification and protein expression was evaluated, we observed a statistical significant correlation between gene amplification and protein expression of cyclin $A$ (correlation coefficient $=0.287, P=0.009$ ) and cyclin $D_{3}$ (correlation coefficient $=0.906, P=4.9 \times 10^{-33}$ ). Protein expression as well as gene amplification of cyclin $A$ was also correlated with gene amplification of other cyclins. When the impact of gene amplification of different cyclins on the patient survival was analysed, only gene amplification of cyclin $A$ was associated with patient survival.

We found a significant interaction between amplification of cyclin A and cyclin $\mathrm{E}$ (Cox regression, $P=0.02$ ). These two cyclins are sequentially time related in the cell cycle. The effect of amplification of cyclin A was therefore tested in a stratified analysis both when the cyclin $E$ gene was not amplified and when the cyclin $E$ gene was amplified. When the cyclin $E$ gene was not amplified, the statistical strength of the cyclin A amplification increased with a HR of 5.5 (95\% confidence interval: 2.2-14.3, $P<0.0001$ ). When cyclin $\mathrm{E}$ was amplified, amplification of cyclin $A$ had no significant impact on survival $(P=0.45)$.

In summary, we have analysed gene amplification and protein expression of both primary and secondary cyclins in invasive breast carcinomas. Overexpression and gene amplification of cyclin A is correlated with gene amplification of other cyclins. Only gene amplification and overexpression of cyclin $A$ was associated with poor prognosis, and amplification of cyclin $A$ is the strongest prognostic factor in patients that have a normal amplicon of cyclin $\mathrm{E}$.

\section{References}

1. Morgan DO: Cyclin-dependent kinases: engines, clocks, and microprocessors. Annu Rev Cell Dev Biol 1997, 13:261-291.

2. Bukholm IR, Bukholm G, Nesland JM: Over-expression of cyclin $A$ is highly associated with early relapse and reduced survival in patients with primary breast carcinomas. Int J Cancer 2001, 93:283-287.

\section{P4.11}

\section{IL-8 is a novel marker for breast cancer}

C Chavey, A Freund, $S$ Durand, G Lazennec

INSERM U540, Montpellier, France

Breast Cancer Research 2005, 7(Suppl 2):P4.11 (DOI 10.1186/bcr1141) Background Estrogen receptor (ER) status is an important parameter in breast cancer management as ER-positive breast cancers have a better prognosis than ER-negative tumors. This difference comes essentially from the lower aggressiveness and invasiveness of ERpositive tumors.

Results Here, we demonstrate that IL- 8 is clearly overexpressed in most ER-negative breast cell lines and breast tumor samples tested. 
We have also performed a large clinical study on breast tumor samples to determine whether IL-8 expression could be correlated with other clinical parameters. In addition, in vitro studies show that the invasion potential of ER-negative breast cancer cells is associated at least in part with expression of IL-8, but not with IL-8 receptor levels. Moreover, IL-8 increases the invasiveness of ER-positive breast cancer cells, thus confirming the invasion-promoting role of IL-8. Overexpression of IL-8 in ER-negative breast cancer cells involves a high transcriptional activity of the IL-8 promoter. By analysing the IL-8 promoter, we have identified the elements responsible for IL-8 overexpression in ERnegative breast cancer cells.

Conclusion Taken together, these results provide the basis for the control of IL-8 expression in breast cancer and define IL-8 as a novel marker of breast cancers.

\section{P4.12}

\section{Lack of evidence for nuclear IGFBP5 in mammary epithelial cells \\ C Berlato', A Jurgeit', S Geley², W Doppler ${ }^{1}$}

${ }^{1}$ Division Medical Biochemistry and ${ }^{2}$ Division Molecular Pathophysiology, Biocenter, Innsbruck Medical University, Innsbruck, Austria

Breast Cancer Research 2005, 7(Suppl 2):P4.12 (DOI 10.1186/bcr1142) Background IGFBP5 plays a role in mediating the effects of IGFs, which are important in mammary gland development [1] and carcinogenesis [2]. IGF-independent effects of IGFBP5 have also been described and it has been postulated that these are at least partially mediated via IGFBP5 localized in the nucleus $[3,4]$.

Methods The cellular localization of IGFBP5 was analyzed by confocal microscopy after either applying exogenous fluorescent-labeled recombinant protein or applying immunostaining of cells ectopically expressing IGFBP5. HC11, MCF10A mammary epithelial and T47D mammary carcinoma cell lines were used in this study.

Results Nuclear localization of IGFBP5 was observed under two conditions: fluorescent-labeled IGFBP5 added to cells with selectively permeabilized plasma but not nuclear membrane; and cells transfected with IGFBP5 expression vectors lacking the coding region for the signal peptide. By contrast, non-permeabilized cells could be stimulated to take up IGFBP5 only into intracellular vesicles outside the nucleus and this was enhanced by adding a conjugate of polylysine and transferrin, indicating an endocytotic uptake route. In addition, cells transfected with IGFBP5 containing the signal peptide secreted IGFBP5 into the medium but did not show any detectable nuclear staining.

Conclusions Nuclear localization of IGFBP5 in mammary epithelial cells required the crossing of the plasma membrane, which does not appear to occur under normal cell culture conditions. Exit of IGFBP5 from endosomal vesicles into the cytosol followed by nuclear uptake was never observed. Our results indicate a minor role or no role of nuclear IGFBP5 in mediating its IGF-independent effect in the mammary epithelium and in breast cancer.

Acknowledgement Supported by the Austrian Science Fund FWF, SFB021 'Cell proliferation and cell death in tumors'.

\section{References}

1. Allan GJ, Beattie J, Flint DJ: The role of IGFBP-5 in mammary gland development and involution. Domest Anim Endocrinol 2004, 27:257-266.

2. Pollak MN, Schernhammer ES, Hankinson SE: Insulin-like growth factors and neoplasia. Nat Rev Cancer 2004, 4:505518.

3. Schedlich L, Le Page SL, Firth SM, Briggs L, Jans DA, Baxter RC: Nuclear import of insulin-like growth factor-binding protein- 3 and $-\mathbf{5}$ is mediated by the importin beta subunit. $J$ Biol Chem 2000, 275:23462-23470.

4. Xu Q, Li S, Zhao Y, Maures TJ, Yin P, Duan C: Evidence that IGF binding protein- 5 functions as a ligand-independent transcriptional regulator in vascular smooth muscle cells. Circ Res 2004, 94:e46-e54.

\section{P4.13}

Identification of clinically relevant gene sets and pathways using functional models of breast tumor suppression

S Seitz', E Korsching ${ }^{2}$, J Weimer ${ }^{3}$, A Jacobsen ${ }^{3}$, N Arnold ${ }^{3}$, A Meindl'4, W Arnold 5 , D Gustavus', C Klebig', I Petersen', S Scherneck ${ }^{1}$

${ }^{1}$ Department of Tumor Genetics, Max Delbrueck Center for Molecular Medicine, Berlin, Germany; ${ }^{2}$ Department of Biochemistry/Theoretical Biology, Gerhard-Domagk Institute of Pathology, University Hospital Muenster, Muenster, Germany; ${ }^{3}$ Oncology Laboratory, Gynecology and Obstetrics Clinic, University Hospital Schleswig-Holstein, Kiel, Germany; ${ }^{4}$ Department of Medical Genetics, Ludwig-Maximilians University, Munich, Germany; ${ }^{5}$ Atugen AG, Berlin, Germany; 6 Institute of Pathology, Charité Medical School, Humboldt-University, Berlin, Germany

Breast Cancer Research 2005, 7(Suppl 2):P4.13 (DOI 10.1186/bcr1143)

Several lines of evidence suggest that chromosome 8 is likely to harbor tumor suppressor gene(s) involved in breast cancer. We have shown previously that microcell-mediated transfer of human chromosome 8 into the breast cancer cell line MDA-MB-231 results in reversion of tumorigenicity of these cells and is accompanied by expression changes of a clinically relevant set of genes.

In the present study we demonstrate that the transfer of human chromosome 8 into another breast cancer cell line, CAL51, results in hybrids characterized by suppression of tumorigenicity in vitro and in vivo as compared with the parental cells. Loss of the transferred chromosome 8 results in reappearance of the CAL51 phenotype. Oligonucleotide microarray analysis identified 78 probe sets differentially expressed in the hybrids as compared with CAL51 and the rerevertant cells. The majority of these genes is involved in signal transduction, developmental processes, angiogenesis, cadherin signaling, Wnt signaling or inflammation.

It is of particular interest that the 78-gene signature is also reflected in a panel of breast tumors, lymph node and distant metastases, and is correlated with several prognostic markers including tumor size, grading, metastatic behavior and estrogen receptor status.

As opposed to the corresponding non-tumorigenic phenotypes demonstrated for the MDA-MB-231-derived and CAL51-derived microcell hybrids, the respective differentially expressed genes strongly differ from each other. However, it was of special interest that the majority of genes of both gene sets could be integrated into a similar spectrum of biological processes and pathways.

Our findings provide an experimental system to identify and evaluate genes but, more importantly, signatures of biological processes and pathways involved in the development and/or progression of breast cancer.

\section{P4.14}

Microcell-mediated transfer of chromosome 6 into the breast cancer cell line MDA-MB-231: a specific set of genes is involved in the reversion of the tumorigenic phenotype

J Strissel', S Seitz', W Arnold ${ }^{2}$, J Weimer ${ }^{3}$, A Jacobsen ${ }^{3}$, N Arnold ${ }^{3}, \mathbf{S}$ Scherneck ${ }^{1}$

${ }^{1}$ Department of Tumor Genetics, Max Delbrueck Center for Molecular Medicine, Berlin, Germany; ${ }^{2}$ Atugen AG, Berlin, Germany; ${ }^{3}$ Oncology Laboratory, Gynecology and Obstetrics Clinic, University Hospital Schleswig-Holstein, Kiel, Germany

Breast Cancer Research 2005, 7(Suppl 2):P4.14 (DOI 10.1186/bcr1144)

Background Several cytogenetic studies demonstrated frequent allelic losses at defined regions on chromosome 6 in breast tumors, suggesting the presence of tumor suppressor gene(s) (TSG) contributing to breast cancer (BC) tumorigenesis. Different techniques identified several candidate TSGs on chromosome 6 in BC, but no functional evidence for a TSG function for these genes could so far be 
supplied. In order to identify key genes and elucidate the regulatory pathways that are involved in the development and progression of BC, we combined array-based expression profiling with a powerful functional approach, the microcell-mediated chromosome transfer.

Methods and results An intact copy of chromosome 6 was transferred into the $6 q$ deleted and highly invasive BC cell line MDA-MB-231 using the microcell-mediated chromosome transfer. It was demonstrated by microsatellite allelotyping, $\mathrm{CGH}$ and FISH that the hybrid clones contain two fragments of chromosome 6, spanning 6p22-q14 and $6 q 15-6 q 27$. The transfer of parts of chromosome 6 into the MDA-MB231 cells resulted in reduced anchorage-dependent growth, reduced in vitro invasion and a strongly reduced tumorigenic potential.

In order to identify genes responsible for the observed reversion of the tumorigenic phenotype in MDA-MB-231 cells, differential gene expression between the parental cell line and the hybrid clones was analysed using oligonucleotide micro-arrays (HG-U133; Affymetrix, Santa Clara, CA, USA). The identified set of differentially expressed genes will be presented.

Conclusions Our results provide functional evidence that the suppression of the tumorigenic phenotype of the BC cell line MDA-MB231 is mediated by a specific set of genes regulated by one or more genes on chromosome 6.

\section{P4.15}

\section{Epigenetic silencing of tropomyosin alters transforming growth factor beta control of cell invasion and metastasis}

\section{A Bakin, A Varga, $\mathbf{Q}$ Zheng, A Safina}

Roswell Park Cancer Institute, Buffalo, New York, USA

Breast Cancer Research 2005, 7(Suppl 2):P4.15 (DOI 10.1186/bcr1145)

Background Transforming growth factor beta (TGF- $\beta$ ) is a potent tumor suppressor but it can also enhance tumor metastasis by inducing epithelial to mesenchymal transition, cell migration, and changes in tumor microenvironment. The mechanisms underlying the metastatic switch in TGF- $\beta$ function are not well understood. We have recently reported that TGF- $\beta$ regulates tropomyosin-based actin microfilament fibers [1], which are essential for cell proliferation, morphology and motility [2]. Smads and p38 MAPK mediate induction of tropomyosin and formation of stable actin microfilament fibers (stress fibers), thereby reducing cell motility. Tropomyosin (TM) is a dimeric coil-coiled protein that binds along actin microfilaments forming a head-to-tail polymer. TM stabilizes microfilaments and protects them from the depolymerizing action of gelsolin and cofilin. Importantly, TGF- $\beta$ induction of stress fibers inversely correlated with metastatic behavior of tumor cells. The metastastic breast cancer MDA-MB-231 cell line with active TGF- $\beta$ signaling did not express TM isoforms encoded by the TPM1 gene. DNA demethylating agent increased TPM1 expression. We hypothesized that DNA methylation may suppress TPM1 and tropomyosin-based actin fibers, thereby reducing TGF- $\beta$ control of tumor cell invasion and metastasis. The goals are to define the mechanisms underlying loss of tropomyosin expression and changes in TGF- $\beta$ tumor suppressor function.

Methods RT-PCR and immunoblotting analysis of expression tropomyosin isoforms encoded by TPM1 and TPM2 genes in a panel of normal epithelial (MCF10A, NMuMG) and carcinoma (MCF7, MDAMB-231, MDA-MB-435, A549, SW620, SW480) cell lines. DNA methylation of the TPM1 promoter was analyzed by bisulfite sequencing in normal and cancer breast cell lines. Cell migration/invasion was studied using transwell and wound-healing assays. Actin filaments and focal adhesions were studied by immunofluorescence. The role of TPM1 was studied using inducible Tet-Off MDA-MB-231 cell lines.

Results Both TPM1 and TPM2 genes were expressed in normal and non-metastatic tumor cell lines. In metastatic breast and colon tumor cell lines, however, TPM1 expression was significantly reduced or absent, whereas TPM2 was expressed at low levels. Treatment of metastatic cell lines (MDA-MB-231, MDA-MB-435, SW620) with demethylating agent 5-aza-2'-deoxycytidine (5-aza-dC) increased
TPM1 expression with little effect on TPM2. Importantly, 5-aza-dC treatment of MDA-MB-231 cells restored TGF- $\beta$ induction of TPM1 and formation of stress fibers. Forced expression of TPM1 using the Tet-Off system increased stress fibers in MDA-MB-231 cells and reduced cell migration. A potential $\mathrm{CpG}$ island spanning the TPM1 proximal promoter, exon 1 , and the beginning of intron 1 was identified. Bisulfite sequencing showed significant cytosine methylation in metastatic cell lines that correlated with a reduced expression of TPM1.

Conclusion Tropomyosin-based stress fibers are essential for TGF- $\beta$ control of cell motility and invasion. Epigenetic suppression of TPM1 may alter tumor suppressor function of TGF- $\beta$ and contribute to the acquisition of metastatic phenotype.

\section{References}

1. Bakin AV, Safina A, Rinehart C, Daroqui C, Darbary $H$, Helfman DM: A critical role of tropomyosins in TGF- $\beta$ regulation of the actin cytoskeleton and cell motility in epithelial cells. Mol Biol Cell 2004, 15:4682-4694.

2. Pawlak G, Helfman DM: Cytoskeletal changes in cell transformation and tumorigenesis. Curr Opin Genet Dev 2001, 11:4147.

\section{P4.16}

Hypermethylation of cyclin $D_{2}$ and DAP kinase is associated with the lobular subtype of breast cancer U Lehmann ${ }^{1}$, P Ahrens' ${ }^{1}$, LU Wingen ${ }^{2}$, B Schlegelberger ${ }^{2}$, F Länger ${ }^{1}$, H Kreipe ${ }^{1}$

${ }^{1}$ Institute of Pathology and ${ }^{2}$ Institute of Cell and Molecular Pathology, Medizinische Hochschule Hannover, Germany

Breast Cancer Research 2005, 7(Suppl 2):P4.16 (DOI 10.1186/bcr1146)

Background Promoter hypermethylation is a common inactivation mechanism in the development and progression of neoplastic transformation. For mammary carcinoma numerous genes have been described to be silenced due to aberrant methylation, some of them already in intraductal carcinomas. Much less is known about the association of hypermethylation events with the different histological subtypes of breast cancer.

Methods Genomic DNA was isolated from fresh-frozen and formalinfixed paraffin-embedded biopsies and was treated with bisulfite for subsequent methylation analysis. Altogether 40 lobular breast cancer, 89 ductal breast cancer, and 20 normal breast tissue samples were analyzed. For this purpose, real-time PCR-based quantitative methylation assays were developed for the following genes: p16INK4a, cyclin $\mathrm{D}_{2}$, RASSF1A, GSTp1, RIZ-1, HIN-1, APC, DAP kinase, Twist, and SOCS-1.

Results A stringent threshold for scoring a sample as 'methylated' (mean of the methylation level in the control group plus twice the standard deviation) was established for every gene analyzed. Differences in DNA methylation between ductal and lobular breast cancer concerning frequency, intensity, age dependence and concurrence of hypermethylation were uncovered. The most frequently hypermethylated genes in the whole series of 129 breast cancer specimens were cyclin $\mathrm{D}_{2}(75.2 \%)$, RASSF1A (71.3\%), and $\mathrm{HIN}-1$ (66.7\%). The p16INK4a and the RIZ-1 genes were only rarely methylated. A quantitative analysis of the methylation levels using the Mann-Whitney test revealed a statistical significant association of the methylation of the genes DAP kinase and cyclin $D_{2}$ with the lobular subtype. By contrast, a mere qualitative scoring of methylation data did not reveal any significant differences.

Conclusions The results presented in this study demonstrate that subtype-specific patterns of aberrant gene methylation exist in breast cancer, which will help to elucidate the underlying biological differences. These subtype-specific patterns could only be revealed by using stringent real-time PCR-based quantitative methylation assays. 


\section{P4.17}

\section{Methylation profiling of carcinogenesis-associated} genes in sporadic breast cancer

M Nemtsova', v Zemlyakova', E Kusnetsova', v Strelnikov', L Lyubchenko², D Zaletayev ${ }^{\mathbf{1}}$

${ }^{1}$ Institute of Molecular Medicine at Moscow Medical Academy, Moscow, Russian Federation; ${ }^{2}$ Blokhin Cancer Research Center RAMS, Moscow, Russian Federation

Breast Cancer Research 2005, 7(Suppl 2):P4.17 (DOI 10.1186/bcr1147)

Background Aberrant methylation of normally unmethylated CpG islands has been associated with transcriptional inactivation of defined tumor suppressor genes (TSG) in human cancer. Abnormal methylation therefore serves as an alternative to the genetic loss of a tumor suppressor gene function by deletion or mutation.

Methods Methylation profiling was performed by methyl-sensitive PCR with genes involved in cancerogenesis: RB1, p14, p15, p16, CDH1, MGMT, HIC1, N33, LAMC3 and TGFBR1. Methylation profiles of these genes were obtained for 105 breast cancer (BC) specimens. Five specimens (section material) of the normal mammary gland tissue and peripheral blood lymphocytes of 30 healthy subjects were also investigated.

Results By methylation-sensitive PCR with specific primers we detected no methylation of any investigated genes in control peripheral blood lymphocytes and in five normal breast tissues. High frequencies of promoter methylation were observed for the major TSG involved in controlling the cell cycle through the Cdk-Rb-E2F signaling pathway: $R B 1,17 \% ; p 16,56 \%$. Methylation of both genes was revealed in $12 \%$ of tumors. $p 15$ was methylated in $2 \%$ only. No methylation was observed for the $\mathrm{CpG}$ island of $p 14$. The methylation frequency was rather high in the case of the $\mathrm{CDH} 1$ promoter (37\%), maximal in the case of the HIC1 promoter (79\%), and relatively low in the case of MGMT (8\%) and N33 (9\%). Methylation was shown to be $32.5 \%$ for LAMC3. Laminins contain motifs providing cell growth and differentiation via selective activation of signaling pathways dependent on protein kinase $\mathrm{C}$ and, probably, involved in metastases spreading. We have detected abnormal methylation of the TGFBR1 CpG island in $35 \%$ samples. TGFBR1 takes part in transforming growth factor beta signaling, mainly resulting in inhibition of cell proliferation. None of tumors showed methylation of all genes. No gene was methylated in $11 \%$ of tumors, and at least one gene in $89 \%$ of tumors.

Conclusion We have shown that $\mathrm{CpG}$ methylation in the promoter regions of TSG is common to sporadic BC. Genes with a higher methylation frequency may be included in the BC methylotype. Identification of the genes with a high methylation frequency is a necessary step in characterizing a particular tumor. Along with other molecular genetic markers, the methylation profile may be employed in early diagnostics and prognostication.

\section{P4.18}

\section{Expression profiling of Wnt pathway genes in breast cancer}

K Benhaj', B Gur1', B Bozkurt'2, I Yulug', C Akcali', M Ozturk'

${ }^{1}$ Department of Molecular Biology and Genetics, Bilkent University, Ankara, Turkey; ${ }^{2}$ Second Surgery Unit, Ankara Numune Teaching Hospital, Ankara, Turkey

Breast Cancer Research 2005, 7(Suppl 2):P4.18 (DOI 10.1186/bcr1148)

Background Wnt signaling, initially identified in early embryogenesis of Drosophila, is involved in a large set of cellular processes, including proliferation, differentiation, migration, and apoptosis. Canonical Wnt signaling is involved in cell fate choices, stem cell renewal and differentiation, whereas non-canonical signaling deals with morphological changes and tissue organization. Since the discovery of Wnt-1 as a virally-induced oncogene in mouse mammary tumors, Wnt signaling has become a center of interest in human breast carcinogenesis. Surprisingly, genetic aberrations of Wnt signaling have been revealed in different malignancies including colon cancer, liver cancer and hematological cancer, but not in breast cancer. Thus, epigenetic changes in Wnt signaling, rather than mutations, may be more relevant to breast cancers in humans. However, this aspect of Wnt signaling in breast cancer is not fully understood. We aimed to study the expression of key components of Wnt signaling in breast cancer. Here, we report the preliminary results obtained from our expression profiling studies for all known Wnt ligand, frizzled receptor, co-receptor and Lef/TCF transcription factor genes, using a set of cell lines and primary tumors.

Methods We studied the expression profile of 34 Wnt pathway genes by the RT-PCR technique. RNAs were extracted from a telomeraseimmortalized human mammalian epithelial cell line (HMEC), six breast cancer cell lines and 15 breast tumors. Tumor samples were selected following pathological analysis of fresh-frozen tissue slices. Corresponding cDNAs were synthesized and subjected to PCR amplification using specific pairs of primers. Equal loading of total cDNAs was checked by PCR analysis of the housekeeping gene GAPDH.

Results HMEC cells, used as a normal control, expressed many Wnt signaling genes, including 9/19 (48\%) Wnt ligands, 7/9 (78\%) frizzled receptors, LRP-5, LRP-6, as well as four LEF/TCF transcription factors. Expression patterns of frizzled receptors, LRP-5, LRP-6, and LEF/TCF transcription factors did not show major changes in breast cancer cell lines. The major change in Wnt signaling genes was observed at the level of ligand expression. The expression of Wnt-3a, Wnt-4, Wnt-6, Wnt-8b and Wnt-9a were upregulated in $50 \%$ or more breast cancer cell lines. Conversely, the expression of Wnt-5a, Wnt-9b and Wnt-16 was downregulated. Our ongoing studies with breast tumors indicate that Frizzled-1, Frizzled-2 and Frizzled- 6 expression is also maintained in breast tumors. Moreover, upregulation of Wnt-4 and Wnt-9a, as well as downregulation of Wnt-5a expression, were observed in $79-100 \%$ of tumors.

Conclusion These observations provide evidence for redundant expression of major genes involved in Wnt signaling in both normal and malignant breast cells. The expression of at least nine Wnt genes in HMEC strongly suggests that some Wnt ligands may provide autocrine or paracrine signaling to normal breast epithelial cells. Six Wnt genes were commonly expressed in both HMEC and breast cancer cell lines, suggesting that some Wnt ligands may not be significantly involved in malignant transformation of mammary epithelial cells. On the other hand, malignant cells have upregulated the expression of Wnt-3a, Wnt-4, Wnt-6, Wnt-8b and Wnt-9a genes that may play a positive role in malignancy. Wnt-3a and Wnt-4 are known to display transforming activity in mammary epithelial cells. The function of Wnt-8b in mammalian cells is not well known, but its Xenopus homolog displays strong axis-duplication activity, suggesting that it may also be a transforming Wnt. On the other hand, the expression of Wnt-5a, Wnt-9b and Wnt-16 was switched-off in malignant breast cells. Although the functions of $W n t-9 b$ and $W n t-16$ are not well known, Wnt-5a has been identified as a tumor suppressor in hematological malignancies, and acts as an antagonist of canonical Wnt signaling. Taken together, these results indicate that there is a switch in Wnt ligand expression pattern in breast cancer cells, and that this may provoke a functional switch in Wnt signaling from non-canonical to canonical pathways.

\section{P4.19 \\ Real-time PCR-based expression profiling of BRCA1-induced genes in primary breast tumors B Gur', B Bozkurt ${ }^{2}$, O Konu', S Seckin ${ }^{3}$, IG Yulug'1}

${ }^{1}$ Department of Molecular Biology and Genetics, Bilkent University, Ankara, Turkey; ${ }^{2}$ General Surgery and ${ }^{3}$ Department of Pathology, Ankara Numune Research and Teaching Hospital, Ankara, Turkey Breast Cancer Research 2005, 7(Suppl 2):P4.19 (DOI 10.1186/bcr1149) Background BRCA1 possesses a number of features common to transcriptional regulatory proteins, suggesting that it may regulate the expression of one or more downstream genes. It is important to determine which genes are transcriptionally influenced by BRCA1 in vivo to explain its role in tumor suppression and in cancer development. In our previous study, a BRCA1 overexpression system enabled us to define the genes whose expression levels were induced in MCF-7 
breast cancer cells by using the PCR-dependent suppression subtractive hybridization technique [1].

Herein, we report the preliminary results obtained from our real-time expression profiling of normal-matched primary breast tumors for six genes, three of which were previously reported [1]. The association between the gene expression profiles and histopathological states of these tumors will contribute to the definition of possible diagnostic markers.

Methods Breast tumors were selected following pathological analysis of fresh-frozen tissue sections. RNAs were extracted from 31 normalmatched breast tumor tissues. Synthesized cDNA samples were subjected to real-time PCR using the QuantiTect SYBR green PCR Master Mix with gene-specific primers. GAPDH is used as a housekeeping gene for normalization. The gene expression levels were quantified using the delta-delta Ct method after normalizing each tumor with its normal counterpart.

Results The real-time expression level of $B R C A 1$ was highly correlated with ERBIN and SMG1 (Pearson correlation, Minitab; $n=31$; $r=0.765$ and $r=0.673$, respectively; $P<0.0001$ ). The pairwise correlations of BRCA1 expression with those of RENT2 and OVCA1, but not with OVCA2, were at moderate levels $(r=0.41$ and $r=0.46$, respectively; $P<0.05$ ). Furthermore, primary breast tumors were hierarchically clustered into two major groups based on their real-time gene expression profiles using the CLUSTER program and were visualized by TRIVIEW [2]. Cluster I tumors were characterized by a high-level expression in BRCA1 target genes $\left(n=20 ; 1.52 \pm 0.6, \log _{2}\right)$ and were low grade on average $(37.5 \% \mathrm{I}, 50 \% \mathrm{II}, 12.5 \% \mathrm{III} ; n=16)$. On the other hand, Cluster II included higher grade tumors $(45 \%$ II, $55 \% \mathrm{III} ; n=11$ ) expressing BRCA1 target genes at a lower level $\left(n=11 ;-0.79 \pm 0.7, \log _{2}\right)$. Based on the Mann-Whitney $U$ test, Cluster I and Cluster II were significantly different in terms of their tumor grades $(W=206 ; P=0.0059)$.

Conclusion This study demonstrated that real-time RT-PCR studies provide highly accurate quantitative profiling for marker gene association with tumor subtypes. The mRNA expression of ERBIN, ERBB2/HER2 binding protein, was found to be tightly correlated with that of BRCA1 in primary breast tumors, as found in MCF7 cells ectopically expressing BRCA1 [1]. The OVCA1 tumor suppressor gene (17p13.3) that displays frequent LOH in both ovarian cancer and breast cancer also showed correlation with $B R C A 1$ in primary breast tumors used in our study. A certain degree of expression variability, part of which could be attributable to the variation in tumor grade, exists for the genes used in this study, including BRCA1. Our findings support the view that association of the patients' clinical and pathological parameters with the gene expression profiles of breast tumor samples carries great importance in the classification of tumor subtypes.

Acknowledgements This work has been supported by grants from the Scientific and Technical Research Council of Turkey and L'Oreal for Women in Science - Turkey.

References

1. Atalay A, Crook T, Ozturk M, Yulug IG: Identification of genes induced by BRCA1 in breast cancer cells. Biochem Biophys Res Commun 2002, 299:839-846.

2. Eisen Laboratory [http://rana.lbl.gov/EisenSoftware.htm]

\section{P4.20}

\section{Development of a rapid screening approach for candidate gene sets in cancer} R Wittig', R Salowsky², S Blaich', S Lyer', JS Maa ${ }^{3}$, O Müller², J Mollenhauer', A Poustka ${ }^{1}$

${ }^{1}$ Molecular Genome Analysis, Deutsches Krebsforschungszentrum, Heidelberg, Germany; ${ }^{2}$ Agilent Technologies, Waldbronn, Germany; ${ }^{3}$ Maxim Biotech, San Francisco, California, USA

Breast Cancer Research 2005, 7(Suppl 2):P4.20 (DOI 10.1186/bcr1150)

Background During the past decade, microarray-based gene expression analysis gave rise to a large number of candidate genes for the diagnostics and therapy of cancer. Bioinformatic approaches for certain cancer phenotypes. A synergy between these advances and the development of screening tools for a rapid and reliable screening of marker gene expression represents an important step towards an improved treatment of cancer.

Methods For the semiquantitative expression screening of 11 candidate genes for drug resistance in melanoma, we combined multiplex RT-PCR (mRT-PCR) with subsequent microfluidic fragment analysis.

Results The functionality of this approach was demonstrated by low interexperimental variations of amplicon quantities after endpoint analysis. Applied to RNA samples derived from drug-sensitive and drug-resistant melanoma cell lines, mRT-PCR delivered results qualitatively concordant with data obtained from northern blot analyses and array analyses. A preliminary screen of four additional melanoma cell lines points to $I L 1 B, A P O D$, and CYR61 as interesting candidates for drug-resistance associated genes. First tests using an automated on-chip electrophoresis platform indicate the applicability of this approach for high-throughput measurements.

Conclusion mRT-PCR combined with on-chip electrophoresis reveals a rapid and easy-to-handle method for candidate gene set evaluation from limited amounts of mRNA. Using gene sets indicative for different tumor phenotypes, this procedure may represent an alternative for future cancer diagnostics.

\section{P4.21}

\section{Application of microarray analyses to identify genes involved in radiation-induced fibrosis OK Rødningen 1, J Alsner², T Hastie ${ }^{3}$, J Overgaard², A-L Børresen-Dale ${ }^{1}$}

${ }^{1}$ Department of Genetics, The Norwegian Radium Hospital, Oslo, Norway; ${ }^{2}$ Department of Experimental Clinical Oncology, Aarhus University Hospital, Denmark; ${ }^{3}$ Department of Statistics, Stanford University, Stanford, California, USA

Breast Cancer Research 2005, 7(Suppl 2):P4.21 (DOI 10.1186/bcr1151) Background Among breast cancer patients receiving ionizing radiation (IR) treatment, a subgroup shows adverse long-term effects in the normal tissue. Radiation-induced fibrosis (RIF) is one of the most serious complications, and risk of RIF is a dose-limiting factor in the treatment of breast cancer patients with IR. The mechanisms whereby IR induces RIF are not fully understood. However, several observations indicate that the variation in normal tissue sensitivity and the consequent risk of developing late morbidity may be genetically determined. The aim of this study was to obtain a comprehensive overview of the changes in gene expression after IR and to identify genes that can be used to predict risk of RIF, using microarray analyses.

Materials and methods Normal fibroblasts were achieved from 41 patients treated with postmastectomy radiotherapy in Aarhus, Denmark, from 1978 to 1982 and subsequently evaluated in detail with regard to development of RIF. The fibroblasts were grown to early confluency before they received radiation. Total RNA was isolated both before and after radiation, labelled and hybridized to cDNA microarrays consisting of 15,000 cDNAs and ESTs [1]. Expression profiles were identified using hierarchical cluster analyses [2]. Statistically significant changes in gene expression were identified using significance analysis of microarrays (SAM), and predictive genes were identified using prediction analysis for microarrays (PAM) [3].

Results and conclusion Microarray data were first analyzed in order to identify radiation-responsive genes. While several genes were involved in known IR response pathways such as cell cycling, proliferation and stress, a substantial fraction of the genes were involved in processes not previously associated with IR response. Of particular interest are genes involved in extracellular matrix composition. SAM analyses were also applied to identify genes in which the expression level correlated with the level of fibrosis. PAM analyses identified a limited set of predictive genes that may provide a basis for a diagnostic tool in the identification of patients with adverse responses to radiation, and to improve and optimize radiotherapy at the individual level. 
Acknowledgements Supported by the EMBIO, University of Oslo, Norway and the Danish Cancer Society.

References

1. Norwegian Microarray Consortium - A National FUGE Platform for DNA Microarray Technology [www.mikromatrise.no]

2. Michael Eisen Laboratory, Lawrence Berkeley National Laboratory and University of California at Berkeley [rana.lbl.gov/ EisenSoftware.htm]

3. Health Research and Policy, and Statistics, Stanford University [www-stat.stanford.edu/ tibs]

\section{P4.22}

\section{Gene expression studies in radiation-sensitive cell lines}

\section{J Aarøe1, R Gatti' , A-L Børresen-Dale1, O Rødningen'}

${ }^{1}$ Department of Genetics, The Norwegian Radium Hospital, Os/o, Norway; ${ }^{2}$ Department of Pathology, University of California Los

Angeles School of Medicine, Los Angeles, California, USA

Breast Cancer Research 2005, 7(Suppl 2):P4.22 (DOI 10.1186/bcr1152)

Background Repair of damaged DNA is a highly regulated process in normal tissue. Several human genetic diseases are known to be or suspected to be due to defects in DNA repair or cell cycle control. Some of these patients are radiation sensitive and/or predisposed for cancer as a cause of mutations in genes involved in these cellular pathways. A well-known group of radiation-sensitive patients is the ataxia-telangiectasia (A-T) patients. This disease is caused by mutations in the ATM (A-T mutated) gene, whose gene product is involved in detecting double-strand breaks. In this study we are trying to reveal the cause of radiation sensitivity in a group of radiationsensitive patients having an A-T phenotype without mutations in ATM.

Methods Gene expression studies were conducted using 15k cDNA $(\mathrm{NRH})$ microarrays on lymphoblastoid cell lines obtained from four control individuals ('normal'), four A-T patients and 10 radiationsensitive patients, before and after radiation. Cells were harvested prior to radiation ( 0 hours) and at 2 hours, 8 hours and 24 hours, respectively, after exposure to ionizing radiation. The cell lines were irradiated with a dose of 2.0 Gy. To be able to study possible similarities and differences in the expression patterns between the three groups of cell lines, we used cluster analyses.

Preliminary results The preliminary results suggest that the radiationsensitive patients constitute a heterogeneous group, and that the cause of their radiation sensitivity may be diverse. Conversely, several samples showed consistency in their gene expression patterns, which might reveal relevant genes and unknown pathways. To understand the biological context we need a broader base of comparison. Ongoing experiments include more samples in this study and will hopefully enable us to reveal the cause of the radiation sensitivity in these patients and bring us a step closer to the understanding of early malignancy development.

\section{P4.23}

Breast tumors induced by high-dose radiation display similar genetic profiles

A Broeks, SR de Kemp, W Bakker, LM Braaf, FE van Leeuwen, M Stovall, MK Schmidt, NS Russell, LFA Wessels, LJ van 't Veer The Netherlands Cancer Institute, Amsterdam, The Netherlands

Breast Cancer Research 2005, 7(Suppl 2):P4.23 (DOI 10.1186/bcr1153)

Background Women who received mantle-field irradiation following Hodgkin's lymphoma ( $\mathrm{HL}$ ) have an age-dependent increased risk of developing breast cancer. It has been estimated that approximately $90 \%$ of the breast carcinomas in these patients is a result of their radiation treatment, which makes this series extremely appropriate to determine a potentially radiation-induced genomic profile.

Methods In this study we have used array-comparative genomic hybridization (array-CGH) and gene expression profiling (GEP) technology to assess the genomic and gene expression changes in radiation-induced breast tumors. For genomic profiling we used DNA isolated from paraffin-embedded primary breast tumors of breast following $\mathrm{HL}(\mathrm{BfHL})$ patients $(n=29)$. These DNAs were hybridized to a small custom-designed BAC array containing 180 clones specifically selected on their function in the DNA-damage repair pathway or breast cancer susceptibility. For gene expression profiling, RNA was isolated from fresh-frozen tissue samples of $13 \mathrm{BfHL}$ patients and hybridized on $35 \mathrm{~K}$ human oligo-array as well as from sporadic breast tumors that were included as controls, matched for age at diagnosis and no exposure to radiation.

Results Hierarchical clustering of all the array-CGH data divided the samples into two groups. One cluster consisted of the tumors that had developed in the unprotected area of the breast that received highdose radiation (20-40 Gy) during treatment. These tumors showed a significantly higher frequency of amplifications and deletions than those that had developed in the low-dose radiation (1-10 Gy) breast area and the control breast tumors. We performed supervised classification on the two groups in order to construct a predictor for identification of tumors that occurred in high-dose irradiation or low-dose irradiation fields. A classifier employing 15 BAC clones achieved the best crossvalidation performance, and had an associated validation performance of $91.7 \%$.

Hierarchical clustering of the GEP data was performed using 6111 significantly differently expressed oligomers, which resulted in a clustering of the so-called radiation-induced tumors separate from the sporadic tumors (software program Genesis). A supervised classification strategy of these two groups revealed 50 significant genes that could differentiate low-dose and high-dose radiation $\mathrm{BfHL}$ versus sporadic tumors. Importantly, the 'radiation profile' found with the array-CGH could be confirmed by the GEP data; for example, deleted genomic regions showed downregulation of expression.

Conclusion Our preliminary results indicate that low-dose radiation as well as the high-dose radiation-induced tumors can be distinguished from sporadic tumors on the basis of their genomic and expression profile.

\section{P4.24}

Molecular characterization of breast cell lines: a tool for breast cancer studies

E Charafe-Jauffret ${ }^{1,2}$, C Ginestier ${ }^{1}, F_{\text {Monville }}^{1}$, J Adélaïde ${ }^{1}$, P Finetti', F Bertucci'1,2, L Xerri 1,2, J Jacquemier1, D Birnbaum ${ }^{1}$

${ }^{1}$ Marseille Cancer Institute, UMR599 Inserm/Institut Paoli-Calmettes and ${ }^{2}$ Université de la Méditerranée, UFR de Médecine, Marseille,

France

Breast Cancer Research 2005, 7(Suppl 2):P4.24 (DOI 10.1186/bcr1154)

Background Breast cancer is a complex, heterogeneous disease at the molecular level. Recent advances proposed a new molecular taxonomy of breast cancer that defines molecular subtypes such as luminal or basal-like cancers. Hopefully this may modify breast cancer management. A great part of our knowledge on breast carcinomas is based on studies of breast cancer cell lines (BCC). Although many data are available on BCC lines, less is actually known on their molecular characterization. We have determined the molecular subtype of 31 BCC using DNA microarrays and confirmed their phenotype by immunohistochemistry (IHC) on 'cell microarrays' (CMA).

Methods DNA array results were obtained on an Affymetrix station. CMA was constructed in a paraffin block from an agarose core of cell line pellets. Proteins studied in IHC were: estrogen and progesterone receptors, transcription factors GATA 3 and GATA 4, tyrosine kinase receptors (EGFR, ERBB2, MET), sialomucin MUC1, luminal cytokeratins CK8/18 and CK19, basal cytokeratins CK5/6 and CK14, CALLA receptor (CD10), caveolae receptors CAV1 and CAV2, mesenchymal vimentin and alpha smooth actin.

Results Hierarchical clustering sharply discriminated two groups of BCC. To determine whether a BCC was of 'luminal' or 'basal-like' subtype, hierarchical clustering was done with the subset of genes selected by Sørlie and colleagues to discriminate molecular subtypes of tumors: eight BCC were luminal-like, 12 were basal-like, and 11 were not clearly affected to one subtype. Supervised analysis selected 
the most discriminant genes between basal and luminal subtypes, and corresponding antibodies were tested on CMA when available. The immunohistochemical profile was in accordance with the transcriptional profile.

Conclusion According to the molecular classification of breast tumors, we have determined two different subtypes of cell lines, luminal and basal-like, and isolated a subset of genes that discriminates both subtypes. The DNA array data were confirmed at the protein level. This characterization of breast cell lines provides a powerful tool to study specifically each molecular subtype of breast cancers. The master genes isolated from each group may be new targets for breast cancer management.

\section{P4.25}

\section{Cell differentiation and dominant signaling pathway signatures in the molecular classification of human breast cancer cell lines \\ CA Wilson, J Dering, G Bernardo, HM Rong, C Ginther, R Ferdman, AM Cook, R Finn, DJ Slamon}

David Geffen School of Medicine at UCLA, Los Angeles, California, USA

Breast Cancer Research 2005, 7(Suppl 2):P4.25 (DOI 10.1186/bcr1155) Background Differentiation markers characteristic of multiple cell types in the mammary gland have emerged as a dominant feature in gene expression profiles that segregate primary human breast cancers. Immunohistochemical and mRNA expression profiling studies of large breast cancer cohorts have reproducibly identified a subset of tumors $(\sim 15 \%)$ that express markers characteristic of the basal layer of the mammary gland. This is in contrast to the many human breast cancers that uniformly express luminal markers such as the simple cytokeratins (K8/K18) and appear to originate from transformed luminal epithelial cells. A logical next step is to determine the dominant signaling pathways and genetic defects that drive tumor initiation and progression, and to understand how they are related to cell lineage in each breast cancer subtype. A large number of breast cancer cell lines have been isolated and individually characterized over the past few decades. We and others have begun to comprehensively align these cell lines with primary tumors based on gene expression profiles and other parameters in order to improve the relevance of data obtained from these experimental models for understanding human disease.

Methods We have characterized a panel of 51 breast cell lines for a large number of properties including in vitro and in vivo growth rates, morphology on plastic and in three-dimensional matrices, and sensitivity to estrogens/anti-estrogens. Baseline microarray profiles were generated using Agilent 60-mer oligonucleotide arrays for each cell line. In addition, we have generated 'signature profiles' for selected cell lines in response to exogenous stimulation such as estrogen and TGF- $\beta$. A constraint-based analysis of microarray profiles generated from primary tumors and breast cancer cell lines in combination with response signatures was used to identify candidate genes and pathways that may play dominant roles in the breast cancer subgroups. Results The 51 breast cell line panel was segregated into two roughly equal sized groups comprised of those with dominant luminal features and those with progenitor or non-luminal properties. The top $\sim 600$ genes that distinguish luminal versus non-luminal cell lines were identified. All of the luminal cell lines express ESR1, HER-2 (ERBB2) or both. The non-luminal cell lines express many components of the wnt signaling pathway, including ligands, frizzled receptors and secreted inhibitory proteins. Interestingly, the majority of the non-luminal cell lines express significantly higher levels of the ROR1 receptor tyrosine kinase relative to luminal cells. The non-luminal cell lines further segregate into those with predominantly basal features versus those with mesenchymal features. A dominant feature of the mesenchymal cell lines is evidence of elevated, autocrine TGF- $\beta$ signaling.

Conclusion Based on our characterizations and array data for a large panel of breast cancer cell lines, primary tumors and response signatures, we propose an integrated model for the molecular transduction pathways with cell-type origin, and further resolves the biological and clinical significance of the well-established markers ER and HER-2. The proposed stratification is likely to help explain the wellknown diversity in response of breast cancers to standard therapeutic regimens and, more importantly, may identify appropriate breast cancer subtypes amenable to targeted therapeutics.

\section{P4.26}

\section{Reproducibility of molecular portraits in early stage breast cancer} DSA Nuyten 1,2, HY Chang ${ }^{3}$, PO Brown ${ }^{4}$, MJ Van de Vijver ${ }^{1}$ ${ }^{1}$ Department of Diagnostic Oncology and ${ }^{2}$ Department of Radiation Oncology, The Netherlands Cancer Institute, Amsterdam, The Netherlands; ${ }^{3}$ Program in Epithelial Biology and ${ }^{4}$ Department of Biochemistry and Howard Hughes Medical Institute, Stanford University, Stanford, California, USA

Breast Cancer Research 2005, 7(Suppl 2):P4.26 (DOI 10.1186/bcr1156)

Background Gene expression profiling has been used to identify specific subgroups of breast carcinomas. Perou and Sørlie [1-3] described five subtypes (basal, luminal A and luminal B, ErbB2 and normal-breast like). Here we have categorized the 295 tumors that were previously assessed with the 70 -gene prognosis profile according to these five subtypes.

Methods In 295 stage I and stage II breast carcinomas treated at the Netherlands Cancer Institute, we have obtained gene expression data of 25,000 genes using micro-array analysis. We have used the previously described Intrinsic Gene Set [3] to define basal type, luminal A and luminal B, ErbB2 and normal epithelium-like type tumors (431 of 487 unique genes matched). We have used two different methods to classify the tumors: two-dimensional hierarchical cluster analysis and nearest centroid classification. We have compared the reproducibility by both methods and we have analyzed clinical outcome (distant metastasis-free probability and overall survival) of these 295 patients based for the different classes. The median follow-up is 6.7 years for all patients and 7.8 years for patients alive.

Results Based on hierarchical clustering, the basal subgroup can be easily recognized; the ErbB2 group is reasonably well defined and the luminal $A$ and luminal $B$ groups form a large cluster, with subclusters that have more luminal $A$ or luminal $B$ patients. For the nearest centroid classification we used a correlation threshold of 0.1 to classify patients. One hundred and nine (37\%) patients did not have a correlation of more than 0.1 to one of the five centroids ('unclassifiable'). Forty-five (15.25\%) patients were assigned to the basal group, 39 (13.2\%) ErbB2, 47 (16\%) luminal A, 45 (15.25\%) luminal B and 10 (3.3\%) normal-breast like. The relatively large group of patients that could not be assigned to one of the classes was further analyzed. These tumors appear to represent a relatively homogeneous group that differs from those that can be classified. The ER receptor is positive in 106/109 (120/188 classifiable patients: two-sided Fisher's exact $P<1 \times 10^{-9}$ ) and $80 \%$ of the tumors are histological grade I or grade II $(47 \%$ for classifiable patients; $\left.P<1 \times 10^{-6}\right)$. Not surprisingly, the 10 -year overall survival is higher in these patients as well ( $80 \%$ vs $64 \%$; log-rank: 0.0005). Using predicting analysis of micro-arrays [4], the unclassifiable 'class' could be predicted using 200 genes with an accuracy of $90 \%$ (cross-validation results).

The 10-year metastasis-free probability and overall survival for the subgroups are: basal, 54\% and 46\%; erbB2, 55\% and 56\%; luminal A, $70 \%$ and $83 \%$; luminal B, 56\% and 63\%; and normal-breast like, $67 \%$ and $90 \%$ (overall $P$ value: metastasis-free probability, 0.15 and overall survival, 0.001).

Conclusion In this series of consecutively treated breast cancer patients, the molecular portraits identify patients that differ with respect to prognosis. The relatively high proportion of unclassifiable patients can possibly be explained by both the cross-platform matching, the difference in clinical stage (locally advanced in the original series versus early stage in our patients), and the fact that the original classification was derived from a relatively small series of tumors. The 
subgroup that could not be classified using the intrinsic genes contains mainly ER-positive and grade I or grade II tumors.

References

1. Perou CM, Sørlie T, Eisen MB, van de Rijn M, Jeffrey SS, Rees CA, Pollack JR, Ross DT, Johnsen H, Akslen LA, et al.: Molecular portraits of human breast tumours. Nature 2000, 406:747-752.

2. Sørlie T, Perou CM, Tibshirani R, Aas T, Geisler S, Johnsen H, Hastie $T$, Eisen MB, van de Rijn M, Jeffrey SS, et al.: Gene expression patterns of breast carcinomas distinguish tumor subclasses with clinical implications. Proc Natl Acad Sci USA 2001, 98:10869-10874.

3. Sørlie T, Tibshirani R, Parker J, Hastie T, Marron JS, Nobel A, Deng S, Johnsen H, Pesich R, Geisler S, et al.: Repeated observation of breast tumor subtypes in independent gene expression data sets. Proc Natl Acad Sci USA 2003, 100:8418-8423.

4. Tibshirani R, Hastie T, Narasimhan B, Chu G: Diagnosis of multiple cancer types by shrunken centroids of gene expression. Proc Natl Acad Sci USA 2002, 99:6567-6572.

\section{P4.27}

\section{Subclassification and molecular characterization of early stage breast carcinomas using Applied Biosystems Human Genome Survey Microarrays Y Wang', T Sørlie², C Xiao³, L-A Macalik', H Johnsen², G Schroth', A-L Børresen-Dale' ${ }^{2}$, R Samaha'}

${ }^{1}$ Applied Biosystems, Foster City, California, USA; ${ }^{2}$ Department of Genetics, The Norwegian Radium Hospital, Montebello, Oslo, Norway; ${ }^{3}$ Celera Genomics, Rockville, Maryland, USA

Breast Cancer Research 2005, 7(Suppl 2):P4.27 (DOI 10.1186/bcr1157)

Background Gene expression profiling has been used to define molecular phenotypes of complex diseases such as breast cancer. The Luminal $A$ and Basal subtypes have been repeatedly identified and validated as the two main subtypes out of the total of five of breast tumors originally identified by Perou and colleagues [1]. These two subtypes of breast tumors have also been associated with a significant difference in clinical outcome: the Luminal A subtype patients have been correlated with a significantly longer overall survival or they lived considerably longer before experiencing relapse or metastatic disease, whereas patients with Basal subtype tumors showed the shortest overall survival time or experienced much shorter disease-free time intervals $[2,3]$. To further substantiate the prognostic value of such expression-based phenotypes in diagnosis/prognosis of breast cancer, we report here an extended study on identification and molecular characterization of clinically relevant subtypes in early stage breast carcinomas.

Methods In this study, we profiled 20 biopsy tissues from early stage breast carcinomas using the Applied Biosystems Human Genome Survey Microarrays, which is a relatively new array platform containing 31,700 60-mer oligonucleotide probes representing a set of 27,868 individual human genes, with single-color chemiluminescence detection. To identify the subtypes in these tumors, we first carried out a centroid correlation analysis coupled with an unsupervised hierarchical clustering analysis. We utilized the 'intrinsic' gene list consisting of 534 genes that have been used to define the five subtypes of breast tumors and their core expression centroids in 122 previously published breast tumors samples [3]. Using the mapped 526 intrinsic genes, we computed the Pearson's correlation coefficient of each sample from this study to each of the five centroids and assigned each sample to the subtype to which it showed the highest correlation. As a second approach, we applied a supervised analysis using the 'Nearest Shrunken Centroid classifier' and the PAM software [4]. We took the previously published 122 Norway/Stanford tumor samples and the mapped 526 intrinsic genes as the training set to identify the predictor genes for the five subtypes. We then used this classifier to predict the subtypes of each of the 20 early stage carcinomas analyzed in this study. The same analyses were applied on parallel datasets generated from Stanford cDNA Arrays and Agilent Human Whole Genome Arrays. Welch-ANOVA analysis coupled with
Benjamini and Hochberg False Discovery Rate multiple testing corrections were performed to identify the 'signature' genes that are most differentially expressed between the subtypes. PANTHER ${ }^{\text {TM }}$ protein classification analysis (Applied Biosystems, Foster City, CA, USA) $[5,6]$ and PathArt ${ }^{\mathrm{TM}}$ (Jubilant Biosys Ltd) pathway analysis were carried out to identify molecular mechanisms underlying these 'signature genes'. A minimal set of genes that best discriminated the two identified subtypes were determined using PAM analysis on the combined datasets generated on the three different array platforms.

Results Both unsupervised and supervised analysis identified the two main clinically relevant subtypes of breast cancer, Luminal A (correlated with a relatively good outcome) and Basal-like (correlated with the poorest outcome). The identification of the Luminal A and Basal subtypes in these early stage breast carcinomas was further validated by parallel data generated from Stanford cDNA Arrays and Agilent Human Whole Genome Arrays. Statistical analysis identified 1210 genes as signature genes characterizing the two subtypes of breast cancer. Protein function and biological pathway analysis on these signature genes revealed different molecular mechanisms descriptive of the two expression-based subtypes: signature genes of the Luminal A subtype were over-represented by genes involved in fatty acid metabolism and steroid hormone-mediated signaling pathways, in particular estrogen receptor-signaling, while signature genes of the Basal-like subtype were over-represented by genes involved in cell proliferation and differentiation, the p21-mediated pathway, and the $\mathrm{G}_{1}-\mathrm{S}$ checkpoint of cell cycle signaling pathways. Finally, we identified a minimal set of 59 predictor genes to best discriminate and characterize the Luminal A and Basal subtypes using PAM analysis on the combined data from the three array platforms. These predictor genes were further verified by TaqMan ${ }^{\circledR}$ expression assays.

Conclusions We have identified and validated the two previously defined clinically relevant subtypes, Luminal A and Basal, in early stage breast carcinomas. This finding further substantiates the prognostic value of such expression-defined phenotypes in breast cancer at an earlier stage. Signature genes characterizing these two subtypes also revealed that distinct molecular mechanisms have been preprogrammed at an early stage in the different subtypes of the disease. Our results provide further evidence that these breast tumor subtypes represent biologically distinct disease entities and may require different therapeutic strategies. Finally, validated by multiple gene expression platforms, the set of 59 predictor genes identified in this study define potential prognostic molecular markers for breast cancer.

References

1. Perou CM, Sørlie T, Eisen MB, et al.: Nature 2000, 406:747-752.

2. Sørlie T, Perou CM, Tibshirani R, et al.: Proc Natl Acad Sci USA 2001, 98:10869-10874.

3. Sørlie T, Tibshiranhi R, Parker J, et al.: Proc Natl Acad Sci USA 2003, 100:8418-8423

4. Tibshirani R, Hastie T, Narasimhan B, et al.: Proc Natl Acad Sci USA 2002, 99:6567-6572.

5. PANTHER ${ }^{\text {TM }}$ Classification System [https://panther.appliedbiosystems.com]

6. Thomas PD, Kejariwal A, Campbell MJ, et al:: Nucleic Acids Res 2003, 31:334-341.

\section{P4.28}

Lymph node metastases display gene expression profiles of their primary breast carcinomas B Weigelt', LFA Wessels ${ }^{2}$, AJ Bosma', AM Glas'2, DSA Nuyten ${ }^{3}$, YD He4, H Dai ${ }^{4}$, JL Peterse', LJ van 't Veer 1,2

${ }^{1}$ Division of Experimental Therapy, ${ }^{2}$ Division of Diagnostic Oncology and ${ }^{3}$ Division of Radiotherapy, The Netherlands Cancer Institute, Amsterdam, The Netherlands; ${ }^{4}$ Rosetta Inpharmatics LLC, Seattle, Washington, USA

Breast Cancer Research 2005, 7(Suppl 2):P4.28 (DOI 10.1186/bcr1158)

Background The axillary lymph node status is the most powerful prognostic factor for breast cancer patients to date. The molecular 
mechanisms that control lymph node metastasis, however, remain poorly understood. The aim of our study was to define patterns of genes or gene regulatory pathways that drive breast cancer lymph node metastasis.

Methods We compared the gene expression profiles of 15 primary breast carcinomas and their matching lymph node metastases using microarrays. Furthermore, we analyzed the expression profiles of two primary breast tumors and a metastasis obtained from the same patient.

Results The gene expression profile of a primary breast carcinoma is more similar to its affiliated metastasis than the second primary tumor of the same patient. In general, primary breast carcinomas and lymph node metastases do not differ at the transcriptional level by a common subset of genes. However, subtle differences in the expression of genes involved in extracellular matrix organization and growth factor signaling are detected in individual pairs of matching primary and metastatic tumors. Surprisingly, however, different sets of these genes are either upregulated or downregulated in lymph node metastases.

Conclusions The overall gene expression profiles of primary breast carcinomas are maintained in their lymph node metastases. This similarity in gene expression can be attributed to tumor-intrinsic factors rather than to patient-specific factors. No common denominator for breast cancer lymph node metastasis could be identified, suggesting that breast carcinomas do not use a shared gene set to accomplish lymph node metastasis.

\section{P4.29}

\section{Genome-wide expression profiling of microdissected human breast tumor cells: tumor classification predictive of metastases and clinical outcome U Vinatzer', B Dampier', M Pacher', K Kaserer'2, C Haslinger ${ }^{3}$, N Schweifer ${ }^{3}$, M Schreiber ${ }^{1}$}

${ }^{1}$ Department of Obstetrics and Gynaecology and ${ }^{2}$ Department of Clinical Pathology, Medical University Vienna, Austria; ${ }^{3}$ Boehringer Ingelheim, Austria, Vienna

Breast Cancer Research 2005, 7(Suppl 2):P4.29 (DOI 10.1186/bcr1159) We are analyzing human breast tumors and metastases with Affymetrix U133 Plus 2.0 GeneChips, following isolation of uniform tumor cell populations by laser-capture microdissection and linear amplification of RNA. Genome-wide expression profiles of currently 68 breast carcinomas, eight lymph node metastases, and eight normal breast samples were determined, and were correlated with detailed clinical outcome data to identify new and improved diagnostic marker genes of breast cancer. These marker genes were validated by real-time PCR as well as by IHC and/or FISH on tissue arrays. We identified a set of genes by which these patients could be classified into those with a short overall survival and those with a good prognosis with high accuracy $(P<0.0006)$. The prognostic markers thus identified included the estrogen receptor (ESR1), a known key prognostic marker gene of breast cancer. Defined sets of $<60$ genes each were identified that discriminated significantly $(P<0.01)$ between control patients and patients who develop lymph node, bone, or lung metastases, or other distant metastases. In contrast, $>1000$ genes were differentially expressed in primary tumors progressing to liver metastases, thus identifying a novel breast cancer subclass comprising $\sim 15 \%$ of our cases. Downregulation of $\mathrm{p} 14 \mathrm{~A}$ and $\mathrm{p} 14 \mathrm{~B}$, two homologous proteins of unknown function, was found to be associated with lung metastases. Tumors expressing high levels of $\mathrm{p} 14 \mathrm{~A}$ and $\mathrm{p} 14 \mathrm{~B}$ do not metastasize to the lung, tend to have a low tumor grade, are predominantly hormone receptor-positive, and are associated with a longer overall survival. This metastasis suppressor activity of $\mathrm{p} 14 \mathrm{~A}$ and $\mathrm{p} 14 \mathrm{~B}$ appears to be lung-specific, as no effect on liver metastasis and only a slight delay on bone metastasis formation were observed in tumors expressing high levels of $\mathrm{p} 14 \mathrm{~A}$ or $\mathrm{p} 14 \mathrm{~B}$. We also identified $\sim 75$ genes coregulated with HER2, an RTK oncogene highly relevant to breast cancer therapy. Sixteen out of the 20 genes most closely co-expressed $\left(P<10^{-5}\right)$ are located in the same chromosomal region as HER2, carcinomas. Furthermore, a potential key role in breast cancer progression of the PI3K/mTOR and the WNT signaling pathways was strongly suggested by our expression profiles. We found that stimulation of protein synthesis and cell growth via PI3K, mTOR, and elF4E is the primary function of IGF signaling, and that activation of the WNT pathway in breast tumors significantly correlated with metastases and poor prognosis.

Acknowledgement This work was supported by funds of the Austrian Ministry of Education, Science, and the Arts (Austrian Genome Research Program GEN-AU).

\section{P4.30}

\section{Gene expression signature of hereditary breast} cancer

V Dudaladava1,2, M Jarzab1, J Pamula1, W Pekala1', T Huzarski³, J Lubinski ${ }^{3}$, E Grzybowska', K Lisowska ${ }^{1}$

${ }^{1}$ Department of Tumor Biology, Maria Sklodowska-Curie Memorial Cancer Center and Institute of Oncology, Gliwice, Poland; 2Institute of Genetics and Cytology, NAS of Belarus, Minsk, Belarus; ${ }^{3}$ Pomeranian Medical Academy, Szczecin, Poland

Breast Cancer Research 2005, 7(Suppl 2):P4.30 (DOI 10.1186/bcr1160)

Background Some clinical features of hereditary breast cancer, which develops in the presence of germline mutations in BRCA genes, are different from those of sporadic cases. Within recent years, better understanding of cancer biology and successful classification of tumors into distinct, clinically relevant subgroups were made possible by the methods of global gene expression analysis. Thus, we decided to compare gene expression profiles of hereditary versus sporadic breast cancer and possibly find the molecular basis underlying clinical observations.

Methods Microarray analysis was performed with HG U133 Plus 2.0 (Affymetrix, Santa Clara, CA, USA) oligonucleotide microarrays, allowing detection of 47,500 transcripts. We have so far performed gene expression profiling in 25 tumor samples obtained from 24 patients: 14 patients with hereditary breast cancer (with and without proven BRCA1 mutation) and 10 patients with sporadic breast carcinoma. We also analyzed, as the reference, six normal breast tissues collected from patients with breast cancer.

Results We compared the expression profile in hereditary breast cancer and the normal breast tissue and found 2983 differentially expressed genes (Welch $t$ test, Benjamini-Hochberg False Discovery Rate below 0.01). By identical criteria we found 648 genes that are significantly changed between sporadic breast cancer and normal tissue. The merged list contained 3138 genes showing changed expression between cancer and normal breast tissue, with 493 genes that were common in both comparisons. We further verified which of the 3138 genes exhibit differences between hereditary and sporadic tumors. We found that 42 probe sets show statistically significant differences between these groups (non-parametric Mann-Whitney test, False Discovery Rate < 0.05). However, only one of these genes (PARK7) remained significantly changed (both by parametric and nonparametric approach) between hereditary and sporadic cases, when taking into account all probe sets present on the array. This gene has been shown to be associated with poor prognosis in ER-negative breast cancer by Nagahata and colleagues [1]. Next, we analyzed hereditary and sporadic cancer tissues in subgroups of ER-positive and ER-negative tumors. Interestingly, in the ER-negative group, top genes differentiating between hereditary and sporadic cancers were still functionally related to the estrogen metabolism and signaling. Finally, we verified the signature of hereditary breast cancer published by Hedenfalk and colleagues [2,3] - two lists of genes, characteristic for BRCA1/BRCA2-linked breast cancer and for BRCAx-linked hereditary breast cancer, that in total correspond to 204 probe sets on the U133 2.0 Plus array (ESTs not included). In our dataset the most significant of these genes (TOB1, transducer of ERBB2) exhibited a False Discovery Rate equal to 0.13 , thus not passing the criteria of statistical significance. 
Conclusions We have specified a signature of 42 genes that differentiate between normal breast tissue and breast cancer and simultaneously allow classification of hereditary and sporadic tumors. However, the range of difference between these classes is rather mild and is strongly influenced by the ER status. Taking this into account, together with the fact that in our study group the signature proposed by Hedenfalk and colleagues does not allow for differentiation between sporadic and hereditary cancers, it is clear that further studies on the larger group of cases are necessary.

Acknowledgments The study was supported by the Ministry of Science and Information Society Technologies (grant number PBZKBN-040/P04/2001). VD is a fellow of a Fellowship Program totally supported by the National Cancer Institute - Office for International Affairs, NIH, Bethesda, MD, USA.

\section{References}

1. Nagahata $T$, Onda $M$, Emi $M$, Nagai $H$, Tsumagari $K$, Fujimoto $T$, Hirano A, Sato T, Nishikawa K, Akiyama F, et al.: Expression profiling to predict postoperative prognosis for estrogen receptor-negative breast cancers by analysis of 25344 genes on cDNA microarray. Cancer Sci 2004, 95:218-225.

2. Hedenfalk I, Duggan D, Chen Y, Radmasher M, Bittner M, Simon R, Meltzer P, Gusterson B, Esteller M, Kallioniemi OP, et al.: Gene-expression profiles in hereditary breast cancer. N Engl J Med 2001, 344:539-548.

3. Hedenfalk I, Ringner M, Ben-Dor A, Yakhini Z, Chen Y, Chebil G, Ach R, Loman N, Olsson $\mathrm{H}$, Meltzer $\mathrm{P}$, et al.: Molecular classification of familial non-BRCA1/BRCA2 breast cancer. Proc Natl Acad Sci USA 2003, 100:2532-2537.

\section{P4.31}

\section{Comparison of the expression profile in breast cancer and ovarian cancer \\ M Jarzab', V Dudaladava ${ }^{1,2}$, K Simek $^{3}$}

${ }^{1}$ Department of Tumor Biology, Maria Sklodowska-Curie Memorial

Cancer Center and Institute of Oncology, Gliwice, Poland; 2 Institute of Genetics and Cytology, NAS of Belarus, Minsk, Belarus; ${ }^{3}$ Department of Automatic Control, Silesian University of Technology, Gliwice, Poland

Breast Cancer Research 2005, 7(Suppl 2):P4.31 (DOI 10.1186/bcr1161)

Background Until now, microarray studies exploited the differences between cancer and corresponding normal tissues or the molecular differences between tumor histotypes originating from one tissue. However, a sound understanding of neoplastic transformation and progression will benefit from comparison of tumors originating from diverse tissues, especially if they share some biological or clinical properties. Such analysis may aid to seek novel therapeutic targets, which are tumor-specific rather than tissue-specific.

The aim of our study was to compare the expression profile in breast cancer (BC) and ovarian cancer (OC), two female adenocarcinomas with similar genetic background and comparable chemosensitivity and radiosensitivity.

Methods We compared expression profiles of 21 breast carcinomas and 17 serous ovarian carcinomas. We used the GeneChip U133 2.0 Plus microarray and a standard amplification procedure. We applied two methods of data preprocessing, RMA and MAS5 algorithm, and compared the results (only RMA data are shown).

Results Both preprocessing approaches resulted in a huge difference between BC and OC (4427 genes, False Discovery Rate lower than $0.1 \%)$. To base the comparison on well-described transcripts, we used the signature of neoplastic transformation proposed by Rhodes and colleagues [1] in a large meta-analysis of 40 cancer datasets. From 168 probe sets found on the U133 2.0 array that were corresponding to Rhodes genes, 30 were differentially expressed between $B C$ and $\mathrm{OC}$ (the strongest differences were within KDELR2, PLK1, PPP2R5C, $A C L Y, G 3 B P, M M P 9, T R A 1, H S P D 1)$ and the remaining 138 probe sets did not show differences in expression. The results confirm the Rhodes signature in $\mathrm{BC}$ and $\mathrm{OC}$; however, these genes were not able to ensure the full subdivision of tumors into breast and ovarian (hierarchical clustering). Furthermore, we analyzed the tissue-specific expression of genes that were either uniformly or differentially expressed in $\mathrm{BC}$ versus $\mathrm{OC}$, by comparison with normal tissues (data from GeneAtlas 2.0 [2]).

In the next step, we performed the unsupervised analysis of BC and OC expression profiles. By Singular Value Decomposition we revealed that the samples were divided into three large clusters, which corresponded to two groups of breast carcinomas (BC1 and BC2) and a separate group of ovarian cancers (OC). These groups were properly separated by expression of estrogen receptor probe set ESR1, which was low in $\mathrm{BC} 1$, showed variable and moderate expression in $\mathrm{OC}$ and showed very high expression in BC2. The expression of ESR1 was similar to the ER result in routine clinical test, with the exception of two $\mathrm{BC}$ cases with high ESR1 and negative ER by immunohistochemistry.

Conclusions There is large similarity in expression of neoplastic transformation signature genes between breast and ovarian carcinomas. Thirty genes from this set are differentially expressed between these cancers. The most prominent difference in the gene expression profile of these tumors could be explained by ESR1 gene expression and may be related to the tissue hormonal profile.

References

1. Rhodes DR, Yu J, Shanker K, Deshpande N, Varambally R, Ghosh D, Barrette T, Pandey A, Chinnaiyan AM: Large-scale metaanalysis of cancer microarray data identifies common transcriptional profiles of neoplastic transformation and progression. Proc Natl Acad Sci USA 2004, 101:9309-9314.

2. Su Al, Wiltshire T, Batalov S, Lapp H, Ching KA, Block D, Zhang J, Soden R, Hayakawa M, Kreiman G, et al.: A gene atlas of the mouse and human protein-encoding transcriptomes. Proc Natl Acad Sci USA 2004, 101:6062-6067

\section{P4.32}

\section{Comparative expressed sequence hybridisation} revealed distinct chromosomal regions of differential gene expression in breast cancer subtypes I Vanden Bempt, V Vanhentenrijk, M Drijkoningen, C De Wolf-Peeters

Department of Pathology, University Hospital of KU Leuven, Belgium Breast Cancer Research 2005, 7(Suppl 2):P4.32 (DOI 10.1186/bcr1162) Background A recently developed expression profiling technique, termed comparative expressed sequence hybridisation (CESH), was applied for the study of lymph-node negative breast cancer. CESH allows global detection of chromosomal regions with differential gene expression in a way similar to that of comparative genomic hybridisation [1]. Using $\mathrm{CESH}$, we compared gene expression patterns between three different breast cancer subtypes: invasive lobular carcinoma (ILC), poorly differentiated invasive ductal carcinoma ERBB2-positive (ERBB2-positive IDC) and poorly differentiated invasive ductal carcinoma ERBB2-negative (ERBB2-negative IDC).

Aims We intended to investigate whether different morphological breast cancer subtypes are characterised by distinct gene expression patterns. Furthermore, we aimed to identify chromosomal regions that harbour genes with potential significance in the underlying biological behaviour of these subtypes.

Methods Total RNA was extracted from 24 frozen tissue blocks representing eight ILC cases, eight ERBB2-positive IDC cases and eight ERBB2-negative IDC cases. Reverse-transcribed RNA (cDNA) from four cases of the same subtype was pooled, resulting in the formation of two cDNA pools per subtype. First, both cDNA pools of the same subtype were paired with each other. Second, cDNA pools of different subtypes were compared.

Results Comparing cDNA pools of the same subtype showed no significant differences in gene expression profiling. Most strikingly, CESH was able to discriminate ILC from poorly differentiated IDC by three differentially expressed regions, including relative overexpression at $8 p 21-p 22$ and relative underexpression at $8 q 13-q 23$ and at $16 q 22$. Collation of all CESH data led to the identification of an ERBB2 
signature, comprising relative overexpression at 3q24-q26.3, 17q12$\mathrm{q} 21$ and $20 \mathrm{q} 12-\mathrm{q} 13.1$ and relative underexpression at $8 \mathrm{q} 24.3$.

Interpretation and conclusion CESH has proved useful for the study of lymph-node-negative breast cancer. It highlights regions of differential gene expression that are selectively associated with breast cancer subtypes and supports the hypothesis that ERBB2-positive IDC is a distinct disease entity. Moreover, CESH was able to identify an ERBB2 signature, comprising four chromosomal regions harbouring genes with potential significance in the aggressive behaviour of ERBB2-positive disease.

Reference

1. Lu YJ, Williamson D, Clark J, Wang R, Tiffin N, Skelton L, Gordon $T$, Williams R, Allan B, Jachman A, et al.: Comparative expressed sequence hybridization to chromosomes for tumor classification and identification of genomic regions of differential gene expression. Proc Natl Acad Sci USA 2001, 98:9197-9202.

\section{P4.33}

\section{Gene expression profiling in breast cancer} challenges the existence of intermediate histological grade

C Sotiriou' 1 , P Wirapati' ${ }^{2}$, S Loi' ${ }^{1}$, A Harris ${ }^{3}$, J Bergh 4 , J Smeds 4 , V Praz ${ }^{2}$, P Farmer ${ }^{2}$, B Haibe-Kains ${ }^{1}$, F Lallemand ${ }^{1}$, M Buyse ${ }^{5}$, M Piccart ${ }^{1}$, M Delorenzi $^{2}$

${ }^{1}$ Jules Bordet Institute, Université Libre de Bruxelles, Belgium; ${ }^{2}$ Swiss Institute of Experimental Cancer Research, Swiss Institute of

Bioinformatics, Lausanne, Switzerland; ${ }^{3}$ Cancer Research UK and University of Oxford, John Radcliffe Hospital, UK; ${ }^{4}$ Karolinska Institute, Stockholm, Sweden; ${ }^{5}$ International Drug Development Institute, Brussels, Belgium

Breast Cancer Research 2005, 7(Suppl 2):P4.33 (DOI 10.1186/bcr1163) Background The histological grade $(\mathrm{HG})$ in breast cancer provides important prognostic information. However, its interobserver variability and poor reproducibility, especially for tumours of intermediate grade, have limited its clinical potential. We hypothesized that molecular characterization of the grade may allow for full exploitation of the association between the grade and relapse beyond the ability of traditional grading procedures.

Methods Six datasets totalling about 700 primary breast cancers, mostly publicly available data, were used in the analysis. Gene expression profiles (GEP) from Affymetrix U133A GeneChips were contrasted between HG 1 (low grade) and HG 3 (high grade) tumours on a training set of 64 estrogen-receptor-positive breast cancer samples. A set of genes positively and negatively correlated with grade was identified on this training set and chosen as grade reporting genes. A scoring system called the 'gene-expression grade index' (GGI), which essentially summarizes the grade reporting genes by their average expression level, was introduced. The GGI was applied to patients not used in the gene selection to test its prognostic value.

Results Using $33 \mathrm{HG} 1$ and 31 HG 3 ER-positive breast carcinomas, 112 Affymetrix probe sets were significantly upregulated in grade 3 and 16 in grade 1 , at a stringent and objective cut-off $P$ value of 0.05 for a false discovery count $>0$. These 128 probe sets represent 97 different reporter genes. Quantifying the level of expression of these reporter genes with the GGl, many tumors in the HG 2 (intermediate grade) populations assume values typical for the HG 1 and $H G 3$ groups in the same study. The HG 2 tumors can therefore be naturally split into a 'HG 1 like' group and a 'HG 3 like' group, to which we attribute a gene expression grade (GG) of 1 and 3 , respectively. Their survival curves follow the $\mathrm{GGl}$ and are similar to those of the HG 1 and HG 3 groups, respectively, splitting HG 2 into a good prognosis group and a poor prognosis group (Fig. 1).

Similar observations were made in the different datasets analysed, in untreated as well as in systemically treated patients, and on the three different main types of microarray platforms, with substantial variability in the number of reporter genes available. Almost all known clinicopathological variables were significantly associated with clinical
Figure 1

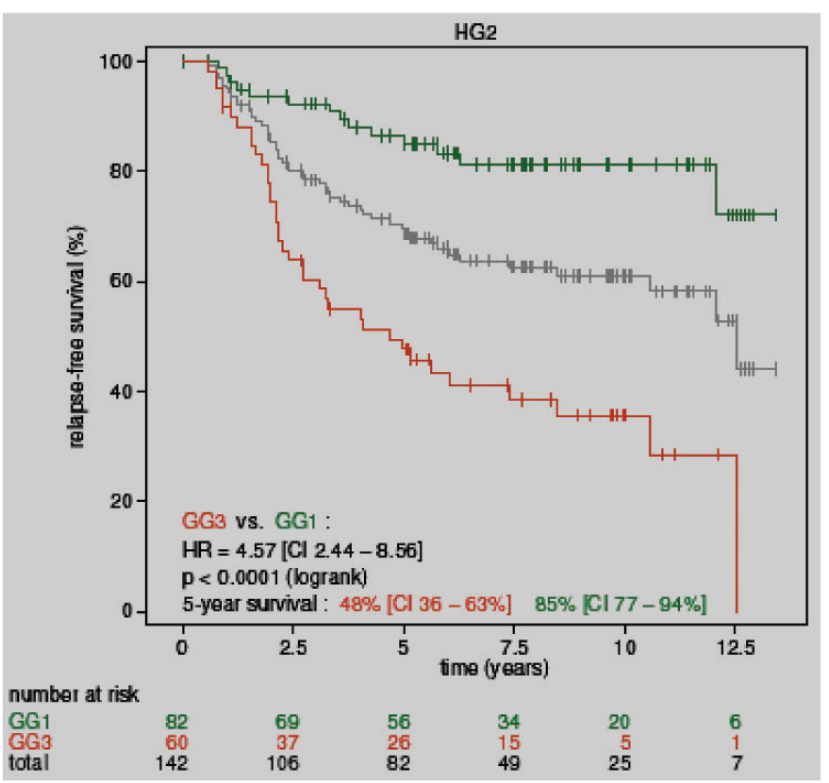

GG, tumour size and nodal status were significant factors. Replacing the $H G$ with the GG significantly improved the prognostic two-group classification obtained with the Nottingham Prognostic Index.

Conclusion Gene-expression-based grading has the potential to significantly improve current grading systems by rendering them more objectively measurable and improving their prognostic value. The superior performance of the two-grade GG system challenges the purpose of classifying tumors as of intermediate grade. Reproduction of these findings in four independent datasets, and across different platforms and with a simple computational system, gives hope that the approach will prove robust and reliable.

\section{P4.34}

Promoter composition predicts gene classes in microarray expression analyses of breast cancer SH Nordgard', T Sørlie', SJ Chanock², A-L Børresen-Dale', K Gardner ${ }^{3}$, VN Kristensen ${ }^{1}$

${ }^{1}$ Department of Genetics, The Norwegian Radium Hospital, Oslo, Norway; ${ }^{2}$ Section on Genomic Variation, Pediatric Oncology Branch, National Cancer Institute, National Institutes of Health, Bethesda, Maryland, USA; ${ }^{2}$ Laboratory of Receptor Biology and Gene Expression and Microarray Facility, Advanced Technology Center, National Cancer Institute, Bethesda, Maryland, USA

Breast Cancer Research 2005, 7(Suppl 2):P4.34 (DOI 10.1186/bcr1164) The human genome contains a large amount of cis-regulatory DNA responsible for directing both spatial and temporal gene-expression patterns. Previous studies have shown that, based on their mRNA expression patterns, breast tumors could be divided into five subgroups (Luminal A, Luminal B, Normal-like, ErbB2+-like, and Basallike), each with a distinct molecular portrait [1]. Whole genome geneexpression analyses of independent sets of breast tumors have revealed repeatedly the robustness of this classification [2]. These patterns have clinical implications in terms of disease-free survival time and are always determined by the same set of genes in all datasets [3]. A list of 552 genes, whose expression in terms of mRNA varied considerably among the different tumors but little between two samples of the same tumor, has been nominated to be sufficient to separate these tumor subgroups. Why exactly these genes? What is the mechanism of their abnormal regulation? Genes are regulated by multiple transcription binding sites that interact with a specific 
combination of transcription factors. Here we report the promoter composition of the genes that strongly predict the patient subgroups. Using a random expectation value (re-value) to generate a background model, we analyzed a total of 277 cis-elements (Genomatix software). The gene classes showed a clear separation when based solely on their promoter composition. This finding suggests that studying those transcription factors associated with the observed expression pattern in breast cancers could identify novel and important biological pathways, including the NF- $\mathrm{KB}$ and Ets transcription factor families.

References

1. Perou, et al:: Nature 2000, 406:747-752.

2. Sørlie, et al.: Proc Natl Acad Sci USA 2003, 100:8418-8423.

3. Sørlie, et al:: Proc Natl Acad Sci USA 2001, 98:10869-10874.

\section{P4.35}

\section{Identification of differentially expressed genes in canine mammary tumor cell lines using a newly developed canine-specific cDNA microarray NAS Rao, $R$ van den Ham, M van Wolferen, JA Mol}

Department of Clinical Sciences of Companion Animals, Faculty of Veterinary Medicine, Utrecht University, The Netherlands

Breast Cancer Research 2005, 7(Suppl 2):P4.35 (DOI 10.1186/bcr1165)

Background Tumors of the mammary gland belong to the most common tumors in the female dog. Ovarian hormones and signaling cascades involving them play an important role in mammary tumor formation and progression in the female dog, as early ovariectomy may reduce the incidence of mammary carcinoma from $30 \%$ to less than $2 \%$. The phenotypic expression of canine mammary tumors may vary, even within one affected animal. In the past, growth factor-independent canine mammary cell lines have been isolated [1]. Molecular characterization of these cell lines and correlation of their molecular signatures to biological behavior may lead to better understanding of signaling pathways involved in mammary cancer and classification of spontaneous mammary tumors.

Methods Three canine mammary tumor cell lines (CMT) originating from primary mammary spindle cell tumor (CMTU309), primary mammary osteosarcoma (CMTU335) and primary mammary anaplastic carcinoma ( $\mathrm{P} 114)$ were compared directly with each other in this study. Cell lines were tested for in vitro invasion using a Transwell assay. Total RNA was isolated from cells grown to near confluence. In vitro transcription followed by labeling and hybridization to a cDNA microarray was carried out according to published protocols [2]. In a loop design of hybridization, labeled cRNA from cell lines were hybridized against each other on a dog-specific cDNA microarray containing 20,160 independent genes, which was developed and spotted in our laboratory. Statistical analysis of microarray data was carried out using significance analysis of microarrays [3]. Further analysis of microarray data was done using GeneSpring.

Results The Transwell invasion assay revealed a clear difference in in vitro invasiveness between canine mammary tumor cell lines. P114 showed a highly invasive phenotype whereas CMTU309 was the least invasive cell line. The cell lines under investigation showed a significant difference in doubling time but no difference in growth factor dependence. Microarray data analysis yielded a total of 451 differentially expressed genes. Among them, about 111 genes were differentially expressed in P114 compared with CMTU335, 110 genes were differentially expressed in P114 against CMTU309 and 230 genes were significantly regulated in CMTU309 against CMTU335. GeneSpring analysis of the microarray data revealed genes unique to each cell line, which were differentially expressed (twofold) in one cell line against the other two cell lines. Unique gene lists containing 19 genes for CMTU309, 62 genes for CMTU335 and 33 genes for P114 were obtained.

Conclusion Our study yielded a novel set of genes unique for each canine mammary cell line in this study. Next, the contributions of these genes, among which some $50 \%$ are not annotated towards phenotypic differences between these cell lines, are under investigation.
Acknowledgement This project is funded by the Morris Animal Foundation, Englewood, Colorado, USA.

References

1. Hellmen E, Moller M, Blankenstein MA, Andersson L, Westermark $B$ : Expression of different phenotypes in cell lines from canine mammary spindle-cell tumors and osteosarcomas indicating a pluripotent mammary stem cell origin. Breast Cancer Res Treat 2000, 61:197-210.

2. Roepman P, Wessels LF, Kettelarij N, Kemmeren P, Miles AJ, Lijnzaad P, Tilanus MG, Koole R, Hordijk GJ, van der Vliet PC, et al:: An expression profile for diagnosis of lymph node metastases from primary head and neck squamous cell carcinomas. Nat Genet 2005, 37:182-186.

3. Tusher VG, Tibshirani R, Chu G: Significance analysis of microarrays applied to the ionizing radiation response. Proc Natl Acad Sci USA 2001, 98:5116-5121.

\section{P4.36}

Discovering genetic profiles by array-CGH in familial breast tumors

PM Nederlof', E van Beers', S Joosse', FBL Hogervorst', LFA Wessels ${ }^{1,2}$, P Devilee ${ }^{3}$, C Cornelisse $^{3}$, R Oldenburg ${ }^{3}$, $S$ Verhoef', LJ van 't Veer'

${ }^{1}$ The Netherlands Cancer Institute, Amsterdam, The Netherlands; ${ }^{2}$ Delft University of Technology, Delft, The Netherlands; ${ }^{3}$ Leiden

University Medical Center, Leiden, The Netherlands

Breast Cancer Research 2005, 7(Suppl 2):P4.36 (DOI 10.1186/bcr1166)

Background We have recently shown that BRCA1 breast tumors can be identified on the basis of their somatic genetic aberrations detected by comparative genomic hybridization $(\mathrm{CGH})$ profiles with high performance (sensitivity: 96\%) [1]. Also, BRCA2 show some specific alterations, but are more similar to sporadic breast tumors [2]. These results illustrate that breast tumors from different genetic backgrounds (BRCA1 and BRCA2) develop different genomic instabilities, and therefore genomic profiles. We hypothesize that this may also be true for BRCAx (BRCA3, BRCA 4, etc.) tumors. We therefore applied CGH to familial breast cancer cases from families without BRCA1/2 mutations.

Aims To produce high-resolution profiles for various types of familial breast cancer, including BRCA1, BRCA2 and BRCAx. To build classifiers based on aCGH profiles. We further aim to optimize class discovery by parallel data analysis of continuous and discrete data as obtained by 'amplicon-finding' algorithms [3]. We also compare BRCA1/2 murine breast tumors with human tumors in an attempt to extract maximal biological meaning from the ploody changes observed in both species [4].

Methods Array-CGH was performed on genomic DNA isolated exclusively from formalin-fixed paraffin-embedded archival breast cancer specimens. Prior to hybridization, multiplex PCR was performed to assess DNA quality. Then, genomic DNA samples were hybridized to a 3500 BAC array [4] representing one clone for each $1 \mathrm{Mb}$ across the human genome.

Results We produced array-CGH profiles for 24 BRCA1 tumors, 16 BRCA2 tumors, 19 control (unselected) tumors and 50 tumors from high-risk families (BRCAx, no BRCA1/2 mutations identified) and show, first, that they reproduce metaphase-CGH profiles. Pronounced alterations included $1 \mathrm{p}$-loss (including the DNA damage response protein FRAP1) in 40\% of tumors of all classes. An extensive region on 1q (including MUC1) shows gain in many tumors but most frequently so (up to $70 \%$ ) in BRCA1 tumors. In a region on $3 p$ (including the tumor suppressor RASSF1), loss was observed in $>40 \%$ of BRCA1 tumors. 3q (including Evi1) was amplified in all tumors classes but most frequently in BRCA1/2 (70\%) compared with controls (20-25\%). $4 p$ loss is significantly more frequent in BRCA1 (45\%) than in either BRCA2 or controls $(10-20 \%)$ and contains a BRCA1 interacting gene, CtBP1. The centromeric region of chromosome 5 shows loss in $10 \%$ of BRCA2, $25 \%$ of CONTR and $45-50 \%$ of BRCA 1 tumors studied. Preliminary analysis of the array-CGH results for the familial breast tumor series, designated BRCAx, show that this is not a 
homogeneous group. Generally, BRCAx profiles present with fewer gains and losses compared with BRCA1, BRCA2 and sporadic breast tumors. This is a finding that needs further quantification and confirmation.

Conclusions Array-CGH can be successfully applied on archival formalin-fixed tumor samples. Array-CGH profiles prove useful in the classification of hereditary (BRCA1) breast tumors. Further data analysis should reveal whether BRCAx can be classified is this manner. We propose the use of array-CGH profiles in clinical genetic counseling and are currently working towards this goal.

Acknowledgement EvB and SJ are funded by the Dutch Cancer Society, NKB.

References

1. Wessels LF, et al:: Molecular classification of breast carcinomas by comparative genomic hybridization: a specific somatic genetic profile for BRCA1 tumors. Cancer Res 2002, 62:71107117.

2. van Beers $E H$, et al.: CGH profiles in human BRCA1 and BRCA2 breast tumors highlight differential sets of genomic aberrations. Cancer Res 2005, 65:822-827.

3. Jong $\mathrm{K}$, et al.: Breakpoint identification and smoothing of array comparative genomic hybridization data. Bioinformatics 2004, 20:3636-3637.

4. Chung YJ, et al:: A whole-genome mouse BAC microarray with $1-\mathrm{Mb}$ resolution for analysis of DNA copy number changes by array comparative genomic hybridization. Genome Res 2004, 14:188-196.

\section{P4.37}

\section{Chromosomal imbalances mapped by array-based comparative genomic hybridization in an integrated approach to combat breast cancer in Denmark J Li, X Zhang, TD Jensen, K Wang, $\mathbf{S}$ Kølvraa, L Bolund}

Institute of Human Genetics, University of Aarhus, Denmark

Breast Cancer Research 2005, 7(Suppl 2):P4.37 (DOI 10.1186/bcr1167) Since its invention by Kallioniemi and colleagues in 1992 [1], comparative genomic hybridization (CGH) has revolutionized the detection and mapping of chromosomal imbalances in neoplasias. However, conventional CGH is handicapped by its low resolution. Array-based CGH brings the resolution towards a molecular level. With a capillary printer we produce arrays on CodeLink slides with 3158 BAC clones, which randomly represent the whole genome. With our homemade arrays, we can detect and map numerical aberrations in a single experiment with about $1 \mathrm{Mb}$ resolution. Furthermore, we have optimized printing, labeling, hybridization, scanning and analysis tools. Reverse-labeling (exchanging the tumor and reference DNA labeling dyes) gives us reliable results even in samples with a substantial admixture of normal cells.

In the Danish Centre for Translational Breast Cancer Research, a 5year project involving 500 high-risk patients is underway. Both prospective and retrospective studies are planned with a systems biological approach involving a multitude of analyses, including arrayCGH. Twenty breast cancer samples have been analyzed in a preliminary study. Chromosome 1q (15/20), chromosome 8 (14/20), chromosome $11(5 / 20)$, chromosome $17 q(9 / 20)$ and chromosome $20 \mathrm{q}(6 / 20)$ gains (duplications and amplifications), and chromosome $22(7 / 20)$ deletions are the most frequent aberrations, which is consistent with the previously published conventional CGH results [2]. Our findings will continuously be integrated with all the other results from the same tumors.

References

1. Kallioniemi A, Kallioniemi OP, Sudar D, Rutovitz D, Gray JW, Waldman F, Pinkel D: Comparative genomic hybridization for molecular cytogenetic analysis of solid tumors. Science 1992, 258:818-821.

2. Stephanie S, Martine DF, Pascale CL: Compilation of published comparative genomic hybridization studies. Cancer Genet Cytogenet 2002, 135:63-90.

\section{P4.38}

\section{Outcome signature genes in breast cancer: is there a} unique set?

\section{Kela, L Ein-Dor, G Getz, D Givol, E Domany}

Department of Physics of Complex Systems and Department of Molecular Cell Biology, Weizmann Institute of Science, Rehovot, Israel Breast Cancer Research 2005, 7(Suppl 2):P4.38 (DOI 10.1186/bcr1168) Predicting the metastatic potential of primary malignant tissues has direct bearing on the choice of therapy. Several microarray studies yielded gene sets whose expression profiles successfully predicted survival. Nevertheless, the overlap between these gene sets is almost zero. One of the main open questions in this context is whether the disparity can be attributed only to trivial reasons such as different technologies, different patients and different types of analysis. To answer this question we concentrated on one single breast cancer dataset, and analyzed it by one single method, that used by van 't Veer and colleagues [1], to produce an outcome predictive signature set of 70 genes. We show that in fact the resulting set of genes is not unique; it is strongly influenced by the subset of patients used for gene selection. Many equally predictive lists could have been produced from the same analysis. Three main properties of the data explain this sensitivity: many genes are correlated with survival; the differences between these correlations are small; and the correlations fluctuate strongly when measured over different subsets of patients. A possible correlation of this finding and the complexity of gene expression in cancer is discussed.

Reference

1. van 't Veer LJ, Dai H, van de Vijver MJ, He YD, Hart AA, Mao M, Peterse HL, van der Kooy K, Marton MJ, Witteveen AT, et al.: Gene expression profiling predicts clinical outcome of breast cancer. Nature 2002, 415:530-536.

\section{P4.39}

\section{Mapping the location of recurring amplicons in array-CGH data}

OC Lingjærde', K Liestøl'1, L Baumbusch², HL Størvold', AL Børresen-Dale ${ }^{2}$

${ }^{1}$ Bioinformatics Group, Department of Informatics, University of Oslo, Norway; ${ }^{2}$ Department of Genetics, Institute for Cancer Research, The Norwegian Radium Hospital, Oslo, Norway

Breast Cancer Research 2005, 7(Suppl 2):P4.39 (DOI 10.1186/bcr1169) Background Copy number alterations (CNAs) are believed to constitute key genetic alterations in the cellular transformation of many tumors [1]. Microarray-based comparative genomic hybridization (array$\mathrm{CGH}$ ) allows the construction of high-resolution genome-wide maps of copy number alterations, and statistical software packages are available for exploring and analysing array-CGH data (see, for example, $[2,3])$, facilitating the delineation of the boundaries of CNAs in individual tumors and thereby localizing and identifying potential oncogenes and tumor suppressor genes. Although CNAs vary widely with respect to size and location, some genomic regions are known to have much higher prevalence of alteration than others. Mapping the location of these CNA hotspots facilitates location of genes of potential importance to tumor development as well as identification of alterations forming key steps in tumor development. There is, however, a need for consistent ways of combining array-CGH results for different arrays. Here, we present a statistical modelling-based approach for this.

Methods Suppose we have available for each gene (clone) on an array a binary (0/1) variable indicating whether the gene is amplified or not. Such data may be constructed from array-CGH data using one of the aforementioned software packages. Each tumor may then be represented by an $m$-dimensional binary vector, where $m$ is the number of genes on the array. For an experiment involving $n$ tumors we thus have a set of $m$-dimensional vectors $z_{1}, \ldots, z_{n}$ and we consider the latter to be realizations from a multivariate distribution $P(z)$. We consider three models for $\mathrm{P}(\mathrm{z})$ of increasing sophistication. The first assumes complete independence between genes, the second 
assumes a Markov-chain dependence structure and the third assumes a Markov Random Field dependence structure [4]. We demonstrate how $\mathrm{P}(\mathrm{z})$ can be estimated in each case and show that, by suitable constrained maximization of $\mathrm{P}(\mathrm{z})$, we may determine genomic intervals corresponding to probable occurring intervals of copy number alteration. Results The method is demonstrated (for all three models) on simulated binary copy number status data for varying number of genes and tumors. We also demonstrate the use on real array-CGH data that have been processed by CGH-Explorer [2] in order to obtain a binary copy number status vectors for each tumor.

Conclusion We have proposed a novel statistical method for the derivation of probable intervals of CNA, based on copy number status data from a sample of tumors. The method is based on a probabilistic model for the copy number status in a tumor, and we have discussed three models of increasing sophistication. The most basic of the three models corresponds to simply reporting all genes that are amplified in at least $k \%$ of the tumors. The other two models take into consideration the important fact that neighboring genes are not, in general, altered independently of each other. Utilizing this property of copy number data allows derivation of probable intervals of CNA that are less prone to noise degradation than alternative methods. In addition, results are derived in the context of a well-defined probabilistic framework and are therefore more easily interpretable.

References

1. Lengauer C, Kinzler KW, Vogelstein B: Genetic instabilities in human cancers. Nature 1998, 396:643-649.

2. Lingjærde OC, Baumbusch LO, Liestøl K, Glad IK, Børresen-Dale AL: CGH-Explorer: a program for analysis of array-CGH data. Bioinformatics 2005, 21:821-822.

3. Wang P, Kim Y, Pollack J, Narasimhan B, Tibshirani R: A method for calling gains and losses in array CGH data. Biostatistics 2005, 6:45-58.

4. Cressie NAC: Statistics for Spatial Data. New York: John Wiley \& Sons; 1993.

\section{P4.40}

\section{Comparative genomic hybridization using} oligonucleotide arrays and total genomic DNA

MT Barrett', N Sampas', A Ben-Dor ${ }^{1}$, A Scheffer ${ }^{1}$, P Anderson ${ }^{1}$, P Tsang ${ }^{1}$, C Gooden ${ }^{2}$, R Walker ${ }^{3}$, B Curry ${ }^{1}$, R Kincaid1', D Lipson', M Bittner' ${ }^{2}$, Y Yakhini', PS Meltzer ${ }^{3}$, L Bruhn' ${ }^{1}, \mathbf{S}$ Laderman $^{1}$

${ }^{1}$ Agilent Technologies, Palo Alto, California, USA; ${ }^{2}$ Translational

Genomics Institute, Phoenix, Arizona, USA; ${ }^{3}$ National Human Genome Research Institute, Bethesda, Maryland, USA

Breast Cancer Research 2005, 7(Suppl 2):P4.40 (DOI 10.1186/bcr1170)

Array-based comparative genomic hybridization (aCGH) measures copy number variations at multiple loci simultaneously, providing an important tool for studying genomic alterations associated with cancer, developmental disorders, and germline copy number polymorphisms. The broadest utility of aCGH is obtained by enabling flexible and highresolution probing of regions of interest while preserving the greatest possible complexity of targets derived from whole genome samples. We therefore developed probe design criteria, assay conditions, and analysis methods that enable 60-mer oligonucleotide arrays to be used for CGH measurements using total genomic DNA [1]. We designed a 60-mer oligonucleotide array with $40 \mathrm{~K}$ probes specifically designed for $\mathrm{CGH}$ representing sequences throughout the human genome with a bias for known and predicted gene loci. We tested the performance of this array for reproducibly measuring and mapping losses, and amplification events of varying levels and sizes using both unamplified and phi29 (Qiagen, Valenica, CA, USA) amplified total genomic DNA from a series of model systems. The mean slope of experimental versus theoretical log-ratios for chromosome $X$ probes on this genome-wide human $\mathrm{CGH}$ array in $\mathrm{XY}$ versus $\mathrm{XX}$ hybridizations typically exceeds 0.9 , with probe by probe error rates of less than $10 \%$ in the separation of their log-ratio distributions. Additionally, we used this platform to examine well-characterized cell lines, including diploid cells with partial deletions in chromosome 18q, and diploid and aneuploid tumor cell lines with known amplification and deletion events. We show that the highly processive DNA polymerase phi29 can be used to prepare aCGH templates from as little as $10 \mathrm{ng}$ starting material that yield highquality aCGH measurements throughout the genome. While phi29 provides a simplified isothermal method for amplifying limiting material, non-specific DNA fragments of high MW are generated in the absence of sufficient input template. Although these products do not hybridize to the array, the presence of these amplification products obscures the accurate quantification of DNA template specific to the input genomic DNA prior to the labeling reaction. To ensure reproducible and robust aCGH assay quality, we developed methods and protocols using the Agilent BioAnalyzer (Agilent Technologies, Palo Alto, CA, USA) to enable accurate quality control for key prehybridization steps, including: phi29 amplification of genomic samples, restriction digestion of templates and target labeling. We have also developed visualization tools and statistically robust computational tools that take into account the estimated errors on the measured log ratios in mapping aberration boundaries, and for identifying common aberrations across multiple samples. We tested the reproducibility of our platform using tumor cell line samples including the colon adenocarcinoma cell line HT29 in hybridizations performed in different laboratories (Agilent Labs, National Human Genome Research Institute, Translational Genomics Institute). We present results, using these methods, demonstrating that in situ synthesized 60-mer oligonucleotide arrays can reproducibly detect genomic lesions including single copy and homozygous deletions, and variable amplicons throughout the genome using full complexity genomic DNA samples.

\section{Reference}

1. Barrett MT, Scheffer A, Ben-Dor A, Sampas N, Lipson D, Kincaid $\mathrm{R}$, Tsang P, Curry B, Baird K, Meltzer PS, et al.: Comparative genomic hybridization using oligonucleotide microarrays and total genomic DNA. Proc Natl Acad Sci USA 2004, 101:1776517770 .

P4.41

DNA copy number changes in breast cancer samples using array-CGH profiling R Argonza-Barrett, W Dorlijn, J Bailey

Agilent Technologies, Inc., Palo Alto, California, USA

Breast Cancer Research 2005, 7(Suppl 2):P4.41 (DOI 10.1186/bcr1171)

Discovering DNA copy number alterations is a major goal in studying susceptibility and the cause of diseases such as cancer. Agilent Technologies' whole genome comparative genomic hybridization array $(\mathrm{aCGH})$ together with highly optimized reagents and protocols are used to generate genome-wide profiles of cancers to help in the identification of causative, diagnostic and prognostic changes. The comparison of the aCGH profile from the breast cancer tumor primary cell line with the aCGH profile of EBV-transformed peripheral blood from the same breast cancer patient showed several regions of DNA loss and gain. These regions were confirmed to correlate with the disease on a panel of flash-frozen breast cancer tumor samples. Using Agilent Technologies' CGHAnalytics software, candidate genes and ESTs have been identified for these regions of interest. Further studies on large breast cancer patient cohorts are required to see whether these candidate genes and ESTs are causative and would have profound prognostic implications.

\section{P4.42}

\section{Quality control of DNA with on-chip electrophoresis for oligonucleotide-array comparative genomic hybridization}

\section{Buhlmann, O Mueller, R Salowsky, S Lightfoot}

Agilent Technologies, Waldbronn, Germany

Breast Cancer Research 2005, 7(Suppl 2):P4.42 (DOI 10.1186/bcr1172) Comparative genomic hybridization (CGH) is an effective tool for the detection of genomic copy number aberrations. Many conditions such as cancer and developmental disorders occur when modifications, 
such as amplifications or deletions, to the genome lead to changes in gene copy number. The use of oligonucleotide-array CGH $(\mathrm{aCGH})$ provides a much more sensitive assay for the detection of genomic variance than traditional $\mathrm{CGH}$ methods.

The Agilent 2100 bioanalyzer and associated RNA assays are now established industry standards for checking the integrity of RNA samples. Furthermore, the on-chip electrophoresis capabilities for DNA analysis play an important role in the quality-control assessment of DNA used in the context of aCGH. One important function of the bioanalyzer is to provide an assessment of the intactness of the input (genomic) DNA. For instance, current DNA amplification methods with $\phi 29$ require an intact template but genomic DNA extracted from formalin-fixed paraffin embedded tissues exhibit a wide range of fragmentation patterns. It is extremely useful to know the quality of the DNA before using it in expensive and time-consuming reactions such as labeling and amplification. Here we look at the use of the bioanalyzer in the context of aCGH quality control, monitoring critical steps in the workflow including DNA amplification, digestion of template and labeling.

\section{P4.43 \\ Predicting survival from gene expression data by generalized partial least squares regression HL Størvold, OC Lingjærde}

Bioinformatics Group, Department of Informatics, University of Oslo, Norway

Breast Cancer Research 2005, 7(Suppl 2):P4.43 (DOI 10.1186/bcr1173)

Background There is considerable interest in linking microarray-based gene expression profiles to clinical endpoint variables such as survival. Standard statistical methodologies typically fail when the number of covariates (genes) far exceeds the number of samples (patients). For example, the standard Cox proportional hazards model cannot be directly applied to data of this form. Several methods have been proposed for dealing with this problem in Cox regression, including partial least squares regression (PLS) [1]. Nguyen and Rocke [2] proposed first applying PLS in order to derive a small set of covariates, and then performing proportional hazards regression on the reduced set of covariates. In their approach, however, PLS is applied to survival times without taking into consideration the fact that the latter may be censored. A further problem with their approach is that the PLS step of their procedure is based on the assumption of a Gaussian (normal) likelihood.

Methods Here, we propose a novel method for combining Cox proportional hazards regression and PLS. This method is a direct generalization of PLS to arbitrary likelihoods, whereas the original PLS method (including that used by Nguyen and Rocke) is designed for Gaussian likelihoods only. Furthermore, in our method PLS is directly integrated with the optimization of the Cox partial likelihood. Specifically, we propose to utilize the equivalence between PLS and a modification of the well-known numerical optimization method called the conjugate gradients (CG) algorithm: applying the modified CG algorithm to a Gaussian likelihood yields PLS. We propose instead to apply the modified CG algorithm to the Cox partial likelihood, hence directly generalizing the PLS algorithm to the Cox likelihood. Our method will take into account the censoring of the outputs, as only the original data will be used during the estimation. Our method also easily generalizes to other likelihoods than the Cox proportional hazards likelihood.

Results We present results from the use of these methods for a dataset containing gene expression data and survival outcome from patients with breast cancer published by Sørlie and colleagues [3].

Conclusion We have presented a method for generalizing PLS that utilizes the equivalence between PLS and the well-known conjugate gradients method. We have applied this method to a Cox partial likelihood to predict survival outcome for patients based on gene expression data. The generalized PLS method presented could easily be applied to other likelihoods as well.
References

1. Lingjærde OC, Christophersen N: Shrinkage structures of partial least squares. Scand J Stat 2000, 27:459-473.

2. Nguyen DV, Rocke DM: Partial least squares proportional hazards regression for application to DNA microarray survival data. Bioinformatics 2002, 18:1625-1632.

3. Sørlie T, Perou CM, Tibshirani R, Aas T, Geisler S, Johansen H, Hastie T, Eisen MB, van de Rijn M, Jeffrey SS, et al.: Gene expression patterns of breast carcinomas distinguish tumor subclasses with clinical implications. Proc Natl Acad Sci USA 2001, 98:10869-10874.

P4.44

Gene expression profiles and the TP53 mutation status are powerful prognostic markers of breast cancer

A Langerød ${ }^{1,2}$, H Zhao ${ }^{3,4}, \varnothing$ Borgan $^{5}$, JM Nesland ${ }^{2,6}$, T Hernandez-Boussard7, IK Bukholm ${ }^{8}$, R Kåresen 9 , AL Børresen-Dale1,2, SS Jeffrey ${ }^{3}$

${ }^{1}$ Department of Genetics, The Norwegian Radium Hospital, Oslo, Norway; ${ }^{2}$ Faculty Division, The Norwegian Radium Hospital, University of Oslo, Norway; ${ }^{3}$ Department of Surgery, Stanford University School of Medicine, Stanford, California, USA; ${ }^{4}$ Present address: Department of Urology, Stanford University School of Medicine, Stanford, California, USA; ${ }^{5}$ Department of Mathematics, University of Oslo, Norway; ${ }^{6}$ Department of Pathology, The Norwegian Radium Hospital, Oslo, Norway; ${ }^{7}$ Department of Biochemistry, Stanford University School of Medicine, Stanford, California, USA; ${ }^{8}$ Department of Surgery, Akershus University Hospital, Nordbyhagen, Norway; ${ }^{9}$ Department of Surgery, Ullevål University Hospital, Oslo, Norway Breast Cancer Research 2005, 7(Suppl 2):P4.44 (DOI 10.1186/bcr1174)

Background Gene expression profiling of breast carcinomas has increased our understanding of the heterogeneous biology of this disease, and promises to impact clinical care. The aim of this study was to evaluate the prognostic value of gene expression-based classification as well as established prognostic markers, including mutation status of the TP53 gene, in a group of breast cancer patients with long-term ( $>10$ years) follow-up.

Methods The clinical and histopathological parameters of 215 breast cancer patients were studied for their effects on clinical outcome using the Kaplan-Meier estimator, the log-rank test and univariate/ multivariate Cox regression. The prognostic impact of mutations in the TP53 gene, identified using TTGE followed by sequencing, was also evaluated. Eighty of the samples were analyzed for gene expression using $42 \mathrm{~K}$ spot cDNA microarrays.

Results Both univariate and multivariate analysis showed that the TP53 mutation status was the strongest predictor of breast cancer survival for these 215 patients, superior to tumor size and nodal status. Hierarchical clustering of gene expression identified four groups of patients with statistically significant survival differences $(P=0.0008)$; 'luminal A' and 'normal-like' subgroups with good prognosis, a 'basallike/ERBB2' group having a very poor outcome within the first 2 years, and a 'luminal non-A' group showing an even poorer prognosis at 10year follow-up. The basal-like/ERBB2 subgroup had a significantly higher frequency of mutations in the TP53 gene than the other subgroups $(P<0.001)$. Adding the gene expression-based classification as a separate parameter in multivariate analysis showed that this classification was an even stronger predictor of outcome than any of the other markers, with TP53 mutations status being approximately equally significant.

Conclusion Our results suggest that gene expression profiles provide additional prognostic information supplementing currently established clinical markers. The results also highlight the role of TP53 as a determinant of the expression profile and as an important prognostic marker of breast cancer. 


\section{P4.45}

\section{TP53 mutations among molecular subtypes of HER2-} positive tumors

Å Helland ${ }^{1,2}, \mathrm{M} \mathrm{Nicolau}^{3}, \mathrm{Y} \mathrm{Ji}^{3}, \mathrm{~A}$ Langerød ${ }^{2}$, DE Sommervoll ${ }^{4}$, A Bergamaschi ${ }^{2}$, JR Pollack ${ }^{5}$, A-L Børresen-Dale ${ }^{2}$, SS Jeffrey ${ }^{3}$ ${ }^{1}$ Department of Oncology, The Norwegian Radium Hospital, Oslo, Norway; '2Department of Genetics, The Norwegian Radium Hospital, Oslo, Norway; ${ }^{3}$ Department of Surgery, Stanford University School of Medicine, Stanford, California, USA; ${ }^{4}$ Department of Research,

Statistics Norway, Os/o, Norway; ${ }^{5}$ Department of Pathology, Stanford University School of Medicine, Stanford, California, USA

Breast Cancer Research 2005, 7(Suppl 2):P4.45 (DOI 10.1186/bcr1175) Background The HER2/ERBB2 receptor tyrosine kinase plays a critical role in the pathogenesis of breast cancer. Amplification and/or overexpression of HER2 occurs in 20-30\% of Caucasian breast cancers and correlates with poor prognosis. However, within the group of patients overexpressing HER2, there are obvious differences regarding the course of the disease. This study identifies different molecular subtypes among 47 HER2-positive tumors based on genome-wide expression profiling. A subset of these tumors was analyzed for TP53 mutations, a gene commonly mutated among breast carcinomas.

Materials and methods RNA isolated from 35 infiltrating ductal breast carcinomas (IDC) with associated HER2 immunohistochemistry (IHC) data were analyzed using 42,000 clone cDNA microarrays. Seventeen of these samples that showed $2+$ or $3+$ protein expression were evaluated by fluorescence in situ hybridization (FISH). Using microarray data from this cohort, we determined an RNA expression threshold associated with IHC/FISH positivity. Twenty-nine IDCs from Norway with TP53 mutation data and whose RNA expression of HER2 exceeded this threshold were added to the subsequent analysis, giving a total of 47 samples defined as HER2-positive. ANOVA correction was used to address differences in methodology and the data were analyzed with hierarchical clustering and disease-specific genomic analysis.

Results At least three molecular subtypes of HER2-positive breast carcinomas were identified by hierarchical clustering of the 18 HER2positive samples based on the $42 \mathrm{~K}$ array data. One subgroup contained tumors that overexpressed estrogen receptor (ER)-associated genes, another subgroup expressed cell-to-cell and cell-to-stroma signaling genes as well as varying amounts of the ER-associated genes, whereas a third subgroup showed no expression of the ER-associated gene cluster. These subgroups were confirmed when analyzing the 47 samples.

TP53 mutation data were available for 32 of the 47 samples and 21 of these had a somatic TP53 mutation. We found less mutations in the ER group, with five mutations and seven wild type, than in the other two groups (four wild type and 16 mutated). This difference was significant $(P=0.034)$

Discussion The TP53 mutations seem to be differentially distributed among the molecular subtypes of HER2-positive tumors. Further studies are required to shed light on the implications of this finding.

\section{P4.46}

\section{Mutant p53 exerts its gain of function through} activation of the NF- $\kappa B$ pathway

A Damalas', L Weis'2, SH Nordgard', VN Kristensen 3 , K Gardner ${ }^{4}$, G Cheng ${ }^{5}$, C Gelinas 6 , M Levrero ${ }^{1,7}$, S Strano ${ }^{1}$, A-L Børresen-Dale ${ }^{3,8}, V_{\text {Rotter }}^{2}$, M Oren ${ }^{2}$, G Blandino'

${ }^{1}$ Department of Experimental Oncology, Regina Elena Cancer Institute, Rome, Italy; ${ }^{2}$ Department of Molecular Cell Biology, The Weizmann Institute of Science, Rehovot, Israel; ${ }^{3}$ Department of Genetics, Institute for Cancer Research, The Norwegian Radium Hospital, Oslo, Norway; ${ }^{4}$ Laboratory of Receptor Biology \& Gene Expression and DHHS/NIHI NCI/CCR, Bethesda, Maryland, USA; ${ }^{5}$ Department of Microbiology, Immunology and Molecular Genetics, University of California at Los Angeles, California, USA; ${ }^{6}$ Center for Advanced Biotechnology and Medicine, Piscataway, New Jersey, USA; ${ }^{7}$ Fondazione Andrea Cesalpino, University La Sapienza, Rome, Italy; ${ }^{8}$ University of Oslo, Faculty Division, The Norwegian Radium Hospital, Oslo, Norway Breast Cancer Research 2005, 7(Suppl 2):P4.46 (DOI 10.1186/bcr1176) The p53 gene is subject to frequent mutations in tumors, often leading to accumulation of excess mutant p53 protein, which can exhibit biological gain of function. In particular, mutant p53 exerts antiapoptotic effects. Likewise, NF- $\mathrm{KB}$ is a potent inhibitor of apoptosis, whose extended activation can promote cancer. We discovered that mutant p53 is in complex with the p65 NF-KB subunit in tumor cells treated with TNF, a potent inducer of NF-KB. In addition, we demonstrated that mutant p53 enhances the transcriptional activity of NF- $\mathrm{KB}$ and its anti-apoptotic efficacy. Moreover, we were able to show that mutant $\mathrm{p} 53$ and NF- $\mathrm{KB}$ are recruited together with the $\mathrm{p} 300$ acetyltransferase to anti-apoptotic target gene promoters. Interestingly, mutant p53 ablation attenuates the activity of NF- $\mathrm{KB}$ and renders cancer cells susceptible to killing by TNF. Finally, we observed a close correlation between the high frequency of p53 mutations and the elevated expression of NF-KB target genes in breast tumors. Therefore, our findings support an important role of $\mathrm{NF}-\mathrm{KB}$ in mediating the oncogenic activities of mutant p53 in tumor cells.

\section{P4.47}

\section{Expression of wild-type and mutated TP53 in breast carcinomas}

LO Baumbusch 1, S Myhre', A Langerød', A Bergamaschi', H Johnsen ${ }^{1}$, S Geisler ${ }^{2}$, PE Lønning ${ }^{2}$, A-L Børresen-Dale ${ }^{1}$

${ }^{1}$ Department of Genetics, Institute for Cancer Research, The Norwegian Radium Hospital, Oslo, Norway; 2Department of Medicine, Section of Oncology, Haukeland University Hospital, Bergen, Norway Breast Cancer Research 2005, 7(Suppl 2):P4.47 (DOI 10.1186/bcr1177) Background The tumor suppressor gene TP53 encodes a transcription factor controlling numerous cellular growth and signaling pathways, and thus it has been entitled the safeguard of genome integrity. The TP53 activity is regulated through several mechanisms including transcription and translation control, protein stability and interaction, and subcellular localization. TP53 reacts to a variety of cellular stress responses. The gene is frequently mutated in human cancers, and cells with inactivated TP53 protein are able to resist cell cycle arrest, DNA repair or apoptosis.

Methods We assessed TP53 mRNA expression in 88 tumor samples from breast cancer patients with locally advanced disease using the real-time quantitative RT-PCR technique. Real-time PCR was carried out on the ABI Prism 7000 sequence detection system (TaqMan; Applied Biosystems, Foster City, CA, USA). All relative quantity values of TP53 mRNA were normalized to the average mRNA levels of two independent endogenous control references.

Results and conclusions The TP53 mRNA intensities in human breast tumors exhibit a specific mutational pattern. In tumors with missense or in-frame mutations mRNA expression levels are significantly elevated compared with wild-type TP53, and in tumors with non-sense, frameshift or splice mutations mRNA expression levels are significantly reduced compared with the wild-type TP53 mRNA expression. Consequently, distinctive groups based on the mRNA expression levels and mutation type can be created as wild type, group I (missense and in-frame mutations) or group II (nonsense, frame-shift and splice mutations). These three groups show significant differences in relation to patient progesterone receptor status, C-ERBB2/HER status, tumor grade, distribution of the different breast cancer subtypes and survival rates.

Interestingly, the TP53 wild type shows a wide range of mRNA expression. The lowest or highest quartiles of wild-type TP53 mRNA expression are significantly correlated to the patient estrogen receptor status, tumor grade and distribution of the different breast cancer subtypes.

Since TP53 interacts with various other tumor suppressor genes and oncogenes, mRNA transcript levels of HDM2, CDC37, C-MYC, and others have been determined, and their relation to the TP53 mRNA expression pattern are under further investigation.

Acknowledgement The present study was supported by The National Programme for Research in Functional Genomics in Norway (FUGE), The Research Council of Norway. 


\section{P4.48}

Independent prognostic value of somatic TP53 gene mutations in 1794 breast cancer patients

M Olivier', A Langerød ${ }^{2}$, P Carrieri ${ }^{3}$, J Bergh ${ }^{4}$, S Klaar 4 , J Eyfjord ${ }^{5}$, C Theillet 6 , C Rodriguez ${ }^{6}$, R Lidereau ${ }^{7}$, I Bièche $^{7}$, J Varley ${ }^{8}$, Y Bignon ${ }^{9}$, N Uhrhammer ${ }^{9}$, R Winqvist ${ }^{10}$, A Jukkola-Vuorinen ${ }^{11}$, D Niederacher ${ }^{12}$, S Kato ${ }^{13}$, C Ishioka ${ }^{13}$, P Hainaut ${ }^{1}$, A-L Børresen-Dale ${ }^{2}$

${ }^{1}$ International Agency for Research on Cancer (CIRC/IARC), Lyon, France; ${ }^{2}$ Department of Genetics, The Norwegian Radium Hospital, and University of Oslo, Faculty Division, The Norwegian Radium Hospital, Oslo, Norway; ${ }^{3}$ INSERM U379, ORS PACA, Epidémiologie sociale appliquée à l'innovation, Marseille, France; ${ }^{4}$ Department of Oncology (Radiumhemmet), Karolinska Hospital and Institute, Stockholm, Sweden; ${ }^{5}$ The Icelandic Cancer Society Molecular and Cell Biology Research Laboratory and Faculty of Medicine, University of Iceland, Reykjavik, Iceland; 6INSERM E 229, CRLC Val d'Aurelle/Paul Lamarque, Montpellier, France; 7 INSERM U735/Oncogénétique, Centre René Huguenin, Saint-Cloud, France; ${ }^{8}$ Paterson Institute for Cancer Research, Manchester, UK; ${ }^{9}$ Department of Oncogenetics, Centre Jean Perrin, Clermont-Ferrand, France; ${ }^{10}$ Department of Clinical Genetics, Oulu University Hospital/University of Oulu, Finland; ${ }^{11}$ Department of Oncology, Oulu University Hospital/University of Oulu, Finland; ${ }^{12}$ Molekulargenetisches Labor, Frauenklinik des Universitätsklinikums Düsseldorf, Germany; ${ }^{13}$ Department of Clinical Oncology, Institute of Development Aging and Cancer, Sendai, Japan

Breast Cancer Research 2005, 7(Suppl 2):P4.48 (DOI 10.1186/bcr1178) Background The prognostic significance of TP53 mutations in breast cancer has been investigated in several studies, but the independency of TP53 towards other prognostic factors and the nature of the mutations that may carry a worse prognosis are still unclear.

Methods Retrospective series of breast cancer cases from 10 hospitals in seven different European countries were combined to assemble a large dataset of clinical and molecular data on 1794 European women with primary breast cancer who were followed-up for 10 years, and whose tumor had been screened for TP53 mutation by gene sequencing. The association between TP53 gene mutation and breast-specific cancer death was examined in univariate and multivariate models including classical prognostic factors of survival. Results TP53 gene mutations were more frequent in tumors of ductal and medullar types, aggressive phenotype (high grade, large size, node-positive cases and low hormone receptor contents) and in women under 60 years old. An elevated risk of breast-specific cancer death within 10 years of follow-up was found in patients with a TP53 mutation within exons 5-8 in their tumor compared with patients with no such mutation (relative risk, 2.27; $P<0.0001$ ). This association remained valid after adjustment for tumor size, nodes status and hormone receptor contents. An interaction between TP53 gene mutation and PR content was found, patients with TP53 mutation and negative PR status having a very bad prognosis independently of tumor size, node status and ER status. More importantly, in patients with PRpositive status, TP53 mutation was associated with a strong reduction in survival over 10 years. Among specific types of TP53 mutations, non-missense mutations and missense mutations in the DNA-binding surface (L2/L3 and LSH motifs) had a worse prognosis than mutations outside the DNA-binding surface. Among missense mutations, those at codon 179 and the R248W mutant were associated with the highest mortality rates.

Conclusion These results clearly show that TP53 gene mutation is an independent factor of prognosis in breast cancer, and advocate its use in clinical practice to improve cancer management.

Acknowledgements This research was supported by EC FP6 funding. This publication reflects the authors' views and not necessarily those of the EC. The EC is not liable for any use that may be made of the information contained herein.
P4.49

\section{Prognostic value genotypes and LOH at TP53 codon} 72 and TP53 mutations in primary breast cancer M Kyndi, 1,2, J Alsner ${ }^{1}$, LL Hansen ${ }^{3}$, FB Sørensen ${ }^{2}$, J Overgaard ${ }^{1}$

${ }^{1}$ Department of Experimental Clinical Oncology and ${ }^{2}$ Department of Pathology, Aarhus University Hospital, Denmark; ${ }^{3}$ Department of Human Genetics, University of Aarhus, Denmark

Breast Cancer Research 2005, 7(Suppl 2):P4.49 (DOI 10.1186/bcr1179) Background Recent data suggest that the Arg72Pro polymorphism of the TP53 gene is correlated to TP53 mutations and prognosis in breast cancer $[1,2]$. In addition, $\mathrm{LOH}$ with retention of the Arg allele has been associated with reduced disease-free survival and overall survival [3].

Patients and methods In the period January 1990-1994 a consecutive cohort of 204 Danish women were diagnosed with primary breast cancer. TP53 mutations were assessed by denaturing gradient gel electrophoresis analysis and DNA sequencing. The Arg72Pro polymorphism was measured in DNA extracted from blood lymphocytes and LOH was measured in DNA extracted from invasive breast carcinomas by a method including PCR, primer extension reactions and denaturing high-performance liquid chromatography analyses [4].

Results Mutations in the TP53 gene in tumour DNA were associated with a significantly higher probability of distant metastases $(P<0.0001)$. The Arg72Pro polymorphism was neither significantly associated with TP53 mutations $(P>0.2)$ nor with probability of distant metastases $(P>0.2)$. Among patients heterozygous at TP53 codon 72 , LOH in tumour tissue was significantly associated with TP53 mutations - with 10 out of 40 patients with LOH carrying a TP53 mutation but only one out of 28 patients with no LOH $(P=0.04)$. However, patients with $\mathrm{LOH}$ at TP53 codon 72 did not have a significantly higher probability of distant metastases as compared with patients with no LOH $(P>0.2)$. But within the group of patients with $\mathrm{LOH}$, a significantly higher probability of distant metastases was found for patients with retention of the Pro allele $(11 / 24)$ as compared with patients with retention of the Arg allele $(2 / 16)(P=0.04)$. Among patients with retention of Pro, five patients out of 24 patients $(21 \%)$ had TP53 mutations as compared with five patients out of 16 patients (31\%) with retention of Arg.

Conclusion Our findings suggest that the Arg72Pro polymorphism is neither associated with TP53 mutations nor with breast cancer prognosis. However, $\mathrm{LOH}$ at codon 72 among heterozygous patients might be associated with TP53 mutations, and patients with retention of the Pro allele might experience a poorer prognosis as compared with patients with retention of the Arg allele.

References

1. Langerod A, Bukholm IR, Bregard A, Lonning PE, Andersen TI, Rognum TO, et al:: The TP53 codon 72 polymorphism may affect the function of TP53 mutations in breast carcinomas but not in colorectal carcinomas. Cancer Epidemiol Biomarkers Prev 2002, 11:1684-1688.

2. Goode EL, Dunning AM, Kuschel B, Healey CS, Day NE, Ponder $\mathrm{BA}$, et al:: Effect of germ-line genetic variation on breast cancer survival in a population-based study. Cancer Res 2002, 62:3052-3057.

3. Bonafe M, Ceccarelli C, Farabegoli F, Santini D, Taffurelli M, Barbi $\mathrm{C}$, et al.: Retention of the $\mathrm{p} 53$ codon $\mathbf{7 2}$ arginine allele is associated with a reduction of disease-free and overall survival in arginine/proline heterozygous breast cancer patients. Clin Cancer Res 2003, 9:4860-4864.

4. Wu G, Hua L, Zhu J, Mo OH, Xu XM: Rapid, accurate genotyping of beta-thalassaemia mutations using a novel multiplex primer extension/denaturing high-performance liquid chromatography assay. Br J Haematol 2003, 122:311-316. 


\section{P4.50}

\section{Evaluation of the arrayed primer extension} resequencing assay for TP53 mutation detection EU Due ${ }^{1}$, H Johnsen 1 , CA Wilson ${ }^{2}$, CJ Fæster ${ }^{1}$, P Vu$^{1}$, A Bergamaschi', P Kringen', AL Børresen-Dale1

${ }^{1}$ Department of Genetics, Institute for Cancer Research, The Norwegian Radium Hospital, Oslo, Norway; 2 UCLA Med-Hematology \& Oncology, Los Angeles, California, USA

Breast Cancer Research 2005, 7(Suppl 2):P4.50 (DOI 10.1186/bcr1180) Over the years we have screened for TP53 mutations in different patient materials using temporal temperature gel electrophoresis (TTGE) [1], followed by direct sequencing of samples with aberrant migrating bands to determine the nature of the sequence alteration. Mutations in the TP53 gene are associated with several different cancer types and have been shown to have both prognostic and predictive implications. In this project we are evaluating whether a commercial available array platform for sequencing the TP53 gene using a primer extension assay (APEX) is as sensitive, rapid and costeffective as TTGE/sequencing.

The array is designed by Asper Biotech [2]. Genomic DNA is amplified by PCR, and dUTP is incorporated. The amplification products are then concentrated and purified with spincolumns. Amplification products are fragmented by Uracil N-glycosylase, and unincorporated dNTPs are inactivated by shrimp alkaline phosphatase. The fragmented PCR products are mixed with thermosequenase and four fluorescencelabelled ddNTPs. The sample mixture is transferred to a chip that contains sequence-specific oligonucleotides. So far, exons 2-9 are included on the array. Genorama ${ }^{\mathrm{TM}}$ Quattrolmager is used for scanning. The Genorama imaging system and genotyping software are used for imaging and semiautomatic sequence analysis.

DNA samples from 48 primary breast carcinomas, 11 ovarian carcinomas and 34 cell lines were used for evaluation. Results from a titration experiment with different ratios of the Arg/Arg and Pro/Pro alleles on codon 72 in the TP53 gene showed that mutations could be detected even if the mutated cells were present in less than $5 \%$. We have experienced that homozygous and hemizygous mutations occasionally are missed by the TTGE technique, but that they all were easily detected by APEX [3]. Detection of deletions and insertions, however, is not yet optimal using the APEX technology and they are frequently missed. For the tumour samples the resequencing efficiency using APEX was $92 \%$ for both DNA strands and $99.5 \%$ for sense and/or antisense strands.

The strength of using the APEX technology is that both strands are simultaneously analyzed, and that no further sequencing is needed. It is rapid and sensitive. Cost-effectiveness is still under evaluation.

References

1. Sørlie T, Vu P, Johnsen H, Lind GE, Lothe RA, Børresen-Dale A-L: Mutation screening of the TP53 gene by temporal temperature gel electrophoresis (TTGE). In: Molecular Toxicology Protocols. Edited by Keohavong P, Grant SG. Totowa, NJ: Humana Press; 2004:207-216. [Methods in Molecular Biology, vol. 291.]

2. Asper Biotech [www.asperbio.com]

3. Kringen P, Bergamaschi A, Due EU, Wang Y, Tagliabue E, Nesland $J M$, Nehman A, Tönisson N, Børresen-Dale A-L: Evaluation of arrayed primer extension (APEX) in TP53 mutation detection in breast and ovarian carcinomas. Biotechniques 2005, in press.

\section{P4.51}

\section{Cancer gene mutation discovery and detection using array-based resequencing} T Tengs 1,2, JC Lee1,2, JG Paez ${ }^{1,3}$, X Zhao1, T LaFramboise', G Giannoukos ${ }^{2}$, RK Thomas ${ }^{1,2}$

${ }^{1}$ Department of Medical Oncology, Dana-Farber Cancer Institute, Harvard Medical School, Boston, Massachusetts, USA; ${ }^{2}$ The Broad Institute of MIT and Harvard, Cambridge, Massachusetts, USA; ${ }^{3}$ Present address: Merck, Boston, Massachusetts, USA Breast Cancer Research 2005, 7(Suppl 2):P4.51 (DOI 10.1186/bcr1181) We set out to determine the feasibility of using microarray-based resequencing for cancer gene mutation screening by designing
GeneChip CustomSeq Resequencing arrays (Affymetrix, Santa Clara, CA, USA) for interrogation of ARAF, BRAF, CDK4, CDK6, CDKN2A, KLF6, HRAS, KRAS, MET, NRAS, PTEN, RAF1, RB1, RET and TP53 (164 exons in total). Arrays also included four intronic bases on either side of the exons to cover splice sites, thus the arrays covered a total of 23,966 bases. Overall performance was very good, with accuracy $>99.99 \%$ and coverage $\sim 97.5 \%$. Twenty NSCLC samples were analyzed using the arrays, and several well-characterized somatic mutations and germline variants were found. The most significant novel finding was the detection of a transforming MET mutation (T1010I) in a NSCLC patient.

\section{P5.01}

Detection of circulating cancer cells in peripheral blood as a prognostic factor in early breast cancer A Fabisiewicz'1, P Kober'1, M Koptyra², E Brewczynska ${ }^{3}$, R Sienkiewicz-Kozlowska ${ }^{3}$, T Pienkowski ${ }^{3}$, JA Siedlecki ${ }^{1}$

${ }^{1}$ Department of Molecular Biology, The Maria Sklodowska-Curie Memorial Cancer Center and Institute of Oncology, Warsaw, Poland; ${ }^{2}$ Center for Biotechnology, College of Science and Technology, Temple University, Philadelphia, Pennsylvania, USA; ${ }^{3}$ Department of Breast Cancer and Reconstructive Surgery, The Maria SklodowskaCurie Memorial Cancer Center and Institute of Oncology, Warsaw, Poland

Breast Cancer Research 2005, 7(Suppl 2):P5.01 (DOI 10.1186/bcr1182) Background Approximately $30 \%$ of patients with localized breast cancer eventually develop distant metastases despite optimal surgery and adjuvant therapies. This fact has been attributed to early tumor seeding via the bloodstream. Therefore, identification of breast cancer cells in blood could enable early detection of micrometastases and could potentially be of prognostic significance. The aim of our study was to evaluate the correlation between circulating breast cancer cells and classical prognostic factors.

Methods From February 1999 until April 2004 blood samples from 81 patients, aged $36-72$, stages I and II, with or without metastases to regional lymph nodes, were collected every 3-6 months. All patients underwent therapeutic surgery and subsequent adjuvant therapies. Blood samples were screened for mRNA encoding hMAM, EGFR, CK-19 and $\beta$-hCG by nested RT-PCR. The result of the test was treated as positive when the expression of one or more mRNA markers was observed in at least two samples taken from the same patient. Clinical data, such as histological grade, pT-grade and pN-grade, menopausal status, estrogen receptor and progesterone receptor status, malignancy grade and others were available for analysis.

Results We found that breast cancer cells were present in peripheral blood of patients even in very early stages of the disease. Any of the two-marker tests used in our experiments, hMAM/ $\beta$-hCG, hMAM/ EGFR or EGFR/ $\beta$-hCG, allowed comparable detection of breast cancer cells: in $68-74 \%$ of lymph node-positive (N1) and $49-59 \%$ of lymph node-negative (NO) patients. Addition of a third marker did not significantly increase detection sensitivity. While analyzing the presence of cancer cells in blood samples collected before mastectomy, we found cancer cells in the blood of only $13 \%$ of NO patients, but in $46 \%$ of $\mathrm{N} 1$ patients. Thirteen of the 81 patients (16\%) experienced recurrence of the disease within a 4-year follow-up period. We did not find any correlation between the presence of cancer cells and recurrence or any other clinical prognostic factors except one: patients with premenopausal status had cancer cells in the blood in $37 \%$ of cases, in comparison with those with postmenopausal status who had cancer cells in $63 \%$ of cases.

Conclusion We have shown that a two-marker RT-PCR assay for hMAM/ $\beta$-hCG, hMAM/EGFR or EGFR/ $\beta$-hCG may be used for detection of occult breast cancer cells in peripheral blood. Except for menopausal status, we did not find any correlation between the presence of cancer cells in the blood and classical prognostic factors. Our data may suggest a different mechanism of disease dissemination in premenopausal and postmenopausal women. 


\section{P5.02}

\section{Detection of minimal disease in breast cancer} AJ Bosma, H Helgason, L Braaf, $\mathbf{S}$ Rodenhuis, LJ van 't Veer The Netherlands Cancer Institute, Amsterdam, The Netherlands Breast Cancer Research 2005, 7(Suppl 2):P5.02 (DOI 10.1186/bcr1183) We investigated the prognostic significance of circulating breast cancer cells in peripheral blood detected by quantitative RT-PCR of marker genes in patients with advanced breast cancer. Blood samples from 94 breast cancer patients with metastatic disease (M1) were examined for circulating tumour cells by studying the mRNA expression of CK19, p1B, PS2, EGP2, mammaglobin and SBEM by real-time PCR. Using a score function, developed for predicting circulating tumour cells by quadratic discriminant analysis (QDA), four expression levels were combined into a single discriminant value. Tumour cells were present in 24 out of 94 (31\%) of the patients, as compared with 0 out of 104 controls. The patients with a positive QDA value did have a progression-free survival at 1 year of $3 \%$ and overall survival at 2 years of $17 \%$, against $22 \%$ and $36 \%$ for patients with a negative QDA value $(P=0.015$ and $P=0.0053$, respectively).

Breast cancer patients with metastatic disease have a significantly worse progression-free survival and overall survival when circulating tumour cells can be detected in their peripheral blood [1]. This method was used but was not sensitive enough to predict survival on the basis of a positive score in peripheral stem cell preparations (PBSC) of patients treated in a high-dose chemotherapy trial. The number of tumour cells in these preparations is too low.

We therefore sought a method that would specifically select breast tumour cells. We now show that a combination of magnetic beads cell separations and one-step cDNA synthesis of mRNA increases the sensitivity by at least 10 -fold. Ten MCF7 cells mixed into $10 \mathrm{ml}$ peripheral blood of a healthy control can be easily identified.

To validate this new method we will analyze blood samples of 200 stage I or stage II breast cancer patients and 50 PBSC of breast cancer patients (and 20 PBSC of patients with lymphoid malignancy as controls). So far, 30 stage I or stage II patients have been evaluated with the marker panel of six genes. A combination of four markers employed in the discriminant score revealed a sensitivity of $10 \%$ in detecting epithelial marker genes in peripheral blood.

The cell separation technique allows at least a 10 -fold increase in sensitivity to detect tumour cell specific mRNA in mixed cell suspensions. Further analysis of the 50 PBSC patients and the prospective 200-patient stage I and stage II diagnostic study will reveal specificity and sensitivity. Interim results indicate an increase in sensitivity of at least $10 \%$.

Reference

1. Weigelt B, Bosma AJ, Hart AAM, Rodenhuis S, van 't Veer $\sqcup$ : Marker genes for circulating tumour cells predict survival in metastasized breast cancer patients. Br J Cancer 2003, 88:1091-1094.

\section{P5.03}

\section{Postoperative serum proteomic profiles and} identification of biomarkers with prognosis value in high-risk early breast cancer patients

A Gonçalves ${ }^{1,2,3}$, B Esterni ${ }^{2}$, F Bertucci' ${ }^{2,3,4}$, R Sauvan 4 , M Cubizolles ${ }^{5}$, S Granjeaud ${ }^{6}$, C Chabannon $^{7}$, G Houvenaegel ${ }^{8}$, J Jacquemier', X-Y Meng ${ }^{5}$, ET Fung ${ }^{5}$, D Birnbaum ${ }^{4}$, D Maraninchi' ${ }^{2,3}$, P Viens ${ }^{2,3}$, JP Borg1

${ }^{1}$ Molecular Pharmacology, ${ }^{2}$ Medical Oncology, ${ }^{4}$ Molecular Oncology, ${ }^{7}$ Biological Resource Center, ${ }^{8}$ Surgical Oncology and ${ }^{9}$ Pathology, Cancer Institute of Marseille, Institut Paoli-Calmettes and UMR599 Institut National de la Santé et de la Recherche Médicale (INSERM), Marseille, France; ${ }^{3}$ University of la Méditerranée, UFR of Médecine, France; ${ }^{5}$ Ciphergen Biosystems, Fremont, California, USA; ${ }^{6}$ TAGC, INSERM-ERM 206, Marseille, France

Breast Cancer Research 2005, 7(Suppl 2):P5.03 (DOI 10.1186/bcr1184) Background A significant number of early breast cancer (EBC) develop metastasis despite standard adjuvant therapy. A better prediction of clinical outcome is needed to optimize and individualize therapeutic decisions.

Methods To identify a protein signature correlating with metastatic relapse, we performed surface-enhanced laser desorption/ionizationtime of flight mass spectrometry profiling of early postoperative serum from 81 high-risk EBC patients. Denatured serum samples were fractionated and the resulting fractions were incubated with ProteinChip arrays (Ciphergen Biosystems, Fremont, CA, USA).

Results Several protein peaks were differentially expressed according to clinical outcome (long-term metastasis-free survival versus metastatic relapse). By combining partial least squares and logistic regression methods, we built a multiprotein model that correctly predicted outcome in $83 \%$ of patients. Consistency and robustness of the model were verified using leave-one-out cross-validation. Five-year metastasis-free survival in 'good prognosis' and 'poor prognosis' patients as defined using the multiprotein index were strikingly different (83\% vs $22 \%, P<0.0001$, log-rank test). In a multivariate Cox regression including conventional pathological factors and multiprotein index, only the latter retained independent prognosis significance for metastatic relapse. Major components of the multiprotein index were identified and included haptoglobin, С3a complement fraction, transferrin, apolipoprotein $C_{1}$ and apolipoprotein $A_{1}$.

Conclusions Postoperative serum protein pattern may have an important prognostic value in high-risk EBC. In addition, it may reveal new insights on the metastatic process while providing new targets for future therapeutics.

\section{P5.04}

Monitoring of minimal residual cancer in bone marrow in high-risk breast cancer patients treated with high-dose chemotherapy

V Drageset', JM Nesland², B Erikstein ${ }^{1}$, E Skovlund 1 , H Sommer ${ }^{3}$, G Anker ${ }^{4}$, E Wist ${ }^{3}$, S Lundgren ${ }^{5}$, J Bergh 6 , G Kvalheim ${ }^{1}$

${ }^{1}$ Laboratory for Cellular Therapy, Department of Medical Oncology and Radiotherapy, The Norwegian Radium Hospital, University of Oslo, Norway; 'Department of Pathology The Norwegian Radium Hospital, Oslo, Norway; ${ }^{3}$ Department of Medical Oncology and Radiotherapy, Ullevål University Hospital, Oslo, Norway; ${ }^{4}$ Department of Medical Oncology and Radiotherapy, Haukeland Hospital, Bergen, Norway; ${ }^{5}$ Department of Oncology, St Olavs Hospital, Trondheim University Hospital, Trondheim, Norway; ${ }^{6}$ Radiumhemmet, Karolinska Institute, Stockholm, Sweden

Breast Cancer Research 2005, 7(Suppl 2):P5.04 (DOI 10.1186/bcr1185) Background The present study aimed to investigate the clinical relevance of minimal residual cancer in breast cancer patients before and after high-dose adjuvant chemotherapy with or without progenitor stem cell support.

Methods One hundred and eighteen high-risk stage II breast cancer patients entering the Scandinavian Study Group multicentre trial [1] were randomised to nine cycles of dose-escalated FEC (5-flurouracil, epirubicin, cyclophosphamide) or three cycles of standard FEC followed by high-dose chemotherapy. Bone marrow (BM) samples at diagnosis and 6 months after completion of chemotherapy were assessed for the presence of cytokeratin-positive $(\mathrm{CK}+)$ cells. CK+ cells in BM were evaluated as a prognostic and predictive marker and were compared with other defined prognostic factors of the primary tumour.

Results Monitoring BM changes at time of diagnosis and at 6 months post-treatment is an independent predictive factor for breast cancerspecific survival ( $P=0.001$, univariate analysis). Those who have consistent CK-negative BM findings constitute a group of patients with good prognosis.

Conclusion Monitoring of CK+ cells in BM before and after high-dose chemotherapy with or without stem cell support can be used clinically as a surrogate maker to predict outcome in breast cancer patients. 
Reference

1. Bergh J, Wiklund $T$, Erikstein B, Lidbrink $E$, Lindman $H$, Malmstrom P, Kellokumpu-Lehtinen P, Bengtsson NO, Soderlund G, Anker G, et al:: Tailored fluorouracil, epirubicin, and cyclophosphamide compared with marrow-supported high-dose chemotherapy as adjuvant treatment for high-risk breast cancer: a randomised trial. Scandinavian Breast Group 9401 study. Lancet 2000, 356:1384-1391.

\section{P5.05}

Hypoxia promotes invasion and metastasis of breast cancer cells by increasing lysyl oxidase expression JT Erler ${ }^{1}$, SS Jeffrey ${ }^{2}$, AJ Giaccia ${ }^{1}$

${ }^{1}$ Stanford University School of Medicine, Department of Radiation Oncology, Stanford, California, USA; ${ }^{2}$ Stanford University Department of Surgery, Stanford, California, USA

Breast Cancer Research 2005, 7(Suppl 2):P5.05 (DOI 10.1186/bcr1186)

Background All solid tumors, including breast cancer, contain areas of low oxygen tension (hypoxia). Hypoxic cells are highly aggressive and metastatic, although the underlying processes remain unclear. We have found lysyl oxidase (LOX) expression to be increased by hypoxia in a variety of human cancer cell types. Lysyl oxidase (LOX) plays an essential role in the formation and maintenance of the extracellular matrix, and has previously been linked to increased in vitro invasion of breast cancer cells [1].

Methods Human breast and cervical cancer cells were deprived of oxygen for 18 hours and the expression levels of LOX were examined by RT-PCR (including quantitative), northern blotting and western blotting. LOX activity was inhibited by antisense or siRNA treatment or by addition of a chemical inhibitor, and the effect on in vitro invasion was examined using Boyden chambers. The in vivo metastatic potential of these cells was also examined in air and hypoxia via tail-vein injection of mice subsequently housed for 4 weeks under $20 \%$ or $10 \%$ oxygen and assessment of lung micro metastases. Previously published microarray datasets were examined for correlation between LOX expression and metastasis in human breast cancer patients $[2,3]$.

Results Incubation of human breast and cervical cancer cells in oxygen-deprived conditions resulted in elevated levels of LOX due to a hypoxia inducible factor 1-dependent increase in mRNA levels. Oxygen-deprived cells demonstrated enhanced in vitro invasion that could be blocked by transfection with LOX antisense oligonucleotides or LOX-specific siRNA, or by treatment with an inhibitor of LOX activity. Cells stably expressing LOX siRNA grew slightly faster in air but demonstrated non-invasive and non-metastatic phenotypes in threedimensional culture, and formed dramatically fewer lung micro metastases in vivo when injected into mouse tail veins, particularly those housed in hypoxic conditions. Analysis of expression data from breast cancer patients revealed a good correlation between LOX and lymph node status (Pearson correlation value of 0.78 ).

Conclusion Our data reveal that hypoxia-induced LOX plays a key role in invasion and metastasis in human breast (and cervical) cancer, and that inhibition of LOX blocks these processes and may enhance effectiveness of therapy. These novel findings suggest that LOX may represent a novel marker of patient prognosis, particularly as an indicator of lymph node status in breast cancer.

References

1. Kirschmann DA, et al.: A molecular role for lysyl oxidase in breast cancer invasion. Cancer Res 2002, 62:4478-4483.

2. Sørlie T, et al.: Gene expression patterns of breast carcinomas distinguish tumor subclasses with clinical implications. Proc Natl Acad Sci USA 2001, 98:10869-10874.

3. Zhao $\mathrm{H}$, et al:: Different gene expression patterns in invasive lobular and ductal carcinomas of the breast. Mol Biol Cell 2004, 15:2523-2536.

\section{P5.06}

Breast tumors induce the recruitment of $\mathrm{AC}^{133^{+}} \mathrm{KDR}^{+}$ endothelial precursor cells mobilized by plasma vascular endothelial growth factor

M Botelho', I Amendoeira2 ${ }^{2}$ J Diamond ${ }^{3}$, S Dias ${ }^{3}$, F Schmitt ${ }^{1,4}$

1IPATIMUP, Institute of Pathology and Molecular Immunology Porto University, Porto, Portugal; ${ }^{2}$ Hospital de S João, Porto, Portugal; ${ }^{3} I P O$, Portuguese Institute of Oncology, Lisbon, Portugal; ${ }^{4}$ Medical Faculty, Porto University, Porto, Portugal

Breast Cancer Research 2005, 7(Suppl 2):P5.06 (DOI 10.1186/bcr1187) Recent evidence has demonstrated the importance of bone marrowderived endothelial precursor cells (EPC) in the contribution to postnatal physiological and pathological neovascularization, and in tumor growth and angiogenesis. These cells are recruited undifferentiated; in response to systemic or chemoattractive signals, such as vascular endothelial growth factor (VEGF), they lodge in the growing or lesioned tissue and differentiate into endothelial cells in response to local stimuli and cell-cell interactions. The extent and the significance of the EPC contribution for the growing of most tumors, including breast carcinomas, are still not defined. We analysed the peripheral blood mononuclear cells (PBMNC) of 48 breast cancer patients and found that $16.7 \%$ of them have circulating EPC. These cells were detected by RT-PCR expression of AC133 and kinase domain receptor (KDR). Furthermore, using an ELISA assay, we also found an association between circulating $\mathrm{AC} 133^{+} \mathrm{KDR}^{+} \mathrm{cells}$ and VEGF plasma levels in these patients. We also found AC133 and KDR positivity in breast carcinoma tissues. To our knowledge, this is the first report addressing the recruitment of EPC to breast tumors. Strategies to impair the mobilization and incorporation of EPC into tumors may interfere with the growth of these tumors.

\section{P5.07}

\section{The extracellular matrix composition and} responsiveness to breast carcinoma therapy

SM Pupa', WS Argraves ${ }^{2}$, S Cotrupi ${ }^{1}$, S Giuffré1, F Castiglioni', S Ménard', E Tagliabue ${ }^{1}$

${ }^{1}$ Molecular Targeting Unit, Department of Experimental Oncology, Istituto Nazionale Tumori, Milan, Italy; ${ }^{2}$ Department of Cell Biology, Medical University of South Carolina, Charleston, South Carolina, USA

Breast Cancer Research 2005, 7(Suppl 2):P5.07 (DOI 10.1186/bcr1188) It is established that stroma surrounding breast carcinoma can be altered in comparison with its normal counterpart, and histological observations recognize lesions with loose stroma rich in hyaluronic acid (HA) and recognize lesions with dense stroma rich in fibulin-1, collagens, laminins, fibronectin and fibrillins. Previous studies have shown that adhesion of tumor cells to different extracellular matrix (ECM) components interferes with drug responses. Thus, to address the functional and biological behavior of the breast cancer-related ECM proteins in response to cytotoxic treatments, the breast carcinoma cell line MDAMB361 was injected into athymic mice in the presence of a matrix containing high levels of fibulin-1, laminin-1 and collagens. The grown tumors displayed significantly $(P=0.01)$ reduced sensitivity to DXR compared with the same cells injected without the matrix, strongly indicating that the ECM milieu of tumor impacts the responsiveness of tumor cells to drugs. The analysis of changes in the ECM components in response to DXR treatment revealed that the human breast carcinoma cell lines SKBR-3, MCF-7, MDAMB157 and MDAMB361 upmodulated fibulin-1 transcript [1] and protein levels, particularly in a form exhibiting a molecular weight (about $50 \mathrm{kDa}$ ) lower than expected (about $74 \mathrm{kDa}$ ), thus suggesting that tumor cells may actively reorganize their matrix environment. In parallel with the increase of fibulin-1 the same stress conditions determined decreased expression of hyaluronan synthetases, the enzymes involved in the synthesis of $\mathrm{HA}$. These preliminary findings provide support for a role of ECM in response to drugs and suggest that reorganization of the ECM following chemotherapy is the basis of drug-induced resistance. 
Acknowledgement Partially supported by the AIRC.

Reference

1. Pupa SM, Ménard S, Forti S, Tagliabue E: New insights into the role of extracellular matrix during tumor onset and progression. J Cell Physio/ 2002, 192:259-267.

\section{P5.08}

\section{Characterization of extracellular matrix composition in breast carcinoma}

A Bergamaschi' ${ }^{1}$, E Tagliabue ${ }^{2}$, T Sørlie ${ }^{1}$, J Nesland ${ }^{3}$, R Orlandi², S Ménard ${ }^{2}$, A-L Børresen-Dale ${ }^{1}$

${ }^{1}$ Department of Genetics, The Norwegian Radium Hospital, Oslo, Norway; ${ }^{2}$ Department of Experimental Oncology, National Cancer Institute, Milan, Italy; ${ }^{3}$ Department of Pathology, The Norwegian Radium Hospital, Oslo, Norway

Breast Cancer Research 2005, 7(Suppl 2):P5.08 (DOI 10.1186/bcr1189)

Background A different view of the tumour as a functional tissue interconnected with the microenvironment has recently been described [1]. Numerous reports in the past years indicate that the growth and progression of breast cancer cells, as well as other tumour cells, depend not only on their malignant potential, but also on stroma components present in the surrounding microenvironment [2]. Recent gene expression profiling studies on breast cancer showed that molecular classification of tumours based on the gene expression patterns can identify clinically different subtypes of cancer with different prognosis or disease outcome $[3,4]$. However, since tumours are functional tissues dynamically interconnected with the microenvironment, this approach could give even more information if the tumor phenotype profile is related to the tumor-surrounding stroma characteristics.

Materials and methods Immunohistochemical staining was carried out for several extracellular matrix (ECM) molecules such as fibronectin, fibulin 1, and laminin in a cohort of 29 formalin-fixed, paraffin-embedded primary breast tumours. Furthermore, evaluation of haematoxylin and eosin sections was performed to classify the surrounding stroma in categories of loose, dense or mixed, respectively.

Gene expression analysis was performed using 22K 60-mer Human $1 \mathrm{~A}$ Oligonucleotide (G4110A) provided by Agilent. A specific extracellular matrix ECM gene list of 282 unique ECM-related genes was created by a basic search in the Human $1 \mathrm{~A}(\mathrm{~V} 2)$ platform on the Agilent website [5] and was used to interrogate the 29 breast cancer transcriptional profiles. To validate and test the robustness of the obtained results, a new dataset of 123 primary breast carcinomas was queried with the ECM gene list.

Results We defined a set of 282 ECM-related genes whose expression separated the tumours in three main groups. We compared the ECM groups, defined by gene expression profile, with the IHC staining results and with stroma categories. Significant correlation between stroma categories and the ECM gene expression profile was retrieved. In contrast, the IHC results were not significantly correlated to the results from with the other methodologies we applied.

Samples were also classified based on breast cancer subtypes published by Sørlie and colleagues [6] using a selected list of 534 genes, known to discriminate tumours in subclasses with clinical implication. In our study, basal-like tumours showed a strong steadiness in clustering in a specific group (ECM1), while the other subtypes were clustering across the three ECM groups.

To validate our data and to correlate the ECM groups to clinical outcome, we utilized the ECM gene list in a new dataset of 123 samples, where long-term follow-up information was available. The new analysis allowed us to identify the same three clusters, indicating the robustness of the ECM classification. Survival analyses showed significantly different outcomes for the patients belonging to the three ECM groups. The ECM1 group was associated with poor overall survival and this was not only related to the high frequency of basal tumors in this group, which are known to display a poor prognosis, since luminal-like tumors were also classified in this group.
Conclusion Gene expression profiling of breast carcinomas allows the identification of three subgroups of tumors according to ECMassociated gene expression. This classification provides new information on breast carcinoma biology and new parameters that may impact both prognosis and prediction of response to therapy.

References

1. Bissell MJ, Radisky D: Putting tumours in a context. Nat Rev Cancer 2001, 1:46-54.

2. Wiseman BS, Werb Z: Stromal effects on mammary gland development and breast cancer. Science 2002, 296:10461049.

3. Perou CM, Sørlie T, Eisen MB, van de Rijn M, Jeffrey SS, Rees CA, Pollack JR, Ross DT, Johnsen H, Akslen LA, et al.: Molecular portraits of human breast tumours. Nature 2000, 406:747-752.

4. Sørlie T, Perou CM, Tibshirani R, Aas T, Geisler S, Johnsen H, Hastie T, Eisen MB, van de Rijn M, Jeffrey SS, et al.: Gene expression patterns of breast carcinomas distinguish tumours subclasses with clinical implications. Proc Natl Acad Sci USA 2001, 98:10869-10874.

5. Agilent Technologies [http://www.chem.agilent.com/cag/bsp/ Arraylnfo.asp]

6. Sørlie T, Tibshirani R, Parker J, Hastie T, Marron JS, Nobel A, Deng S, Johnsen H, Pesich R, Geisler S, et al:: Repeated observation of breast tumor subtypes in independent gene expression data sets. Proc Natl Acad Sci USA 2003, 100:8418-8423.

\section{P5.09}

\section{Brn-3b transcription factor in breast tumourigenesis: regulation of genes associated with growth and migration of cancer cells}

\section{SA Lee, VS Budhram-Mahadeo}

Medical Molecular Biology Unit, Institute of Child Health, University College London, UK

Breast Cancer Research 2005, 7(Suppl 2):P5.09 (DOI 10.1186/bcr1190)

Background The Brn-3b transcription factor is elevated in many breast cancers compared with levels found in normal breast epithelial cells. High levels of Brn-3b increase growth and proliferation of cancer cells, both in vitro and in vivo, but also alter migration and confer resistance to growth inhibitory stimulus [1]. Conversely, low levels of Brn-3b slow the growth of these cells. As a transcription factor, Brn3b changes the growth and behaviour of breast cancer cells by modifying the expression of target genes, either directly or indirectly upon association with the proliferation-associated estrogen receptor (ER) [2]. A number of Brn-3b target genes have been identified that alter the growth and behaviour of these cancer cells. For instance, Brn-3b transactivates the promoter of the cyclin-dependent kinase, CDK4 [3], that is required for cell cycle progression and hence proliferation. Brn-3b also represses the promoter of the tumour suppressor gene, BRCA1, and inversely correlates with BRCA1 protein in tumour biopsies. We have recently demonstrated that the small heat shock protein, HSP27, is also regulated by Brn-3b [4]. High expression of HSP27 in breast cancers is associated with increased anchorage-independent growth, increased invasiveness and resistance to chemotherapeutic drugs and poor prognosis. Thus, in cancers expressing high levels of Brn-3b the downstream target genes regulated by this transcription factor can alter the growth and behaviour of these cells.

Methods Western blot analysis of tumour samples was used to correlate Brn-3b and HSP27 proteins. Transient co-transfection and reporter assays were used to look at the effects of Brn-3b and/or ER on the HSP27 promoter. Short hairpin RNA interference was used to target Brn-3b protein and to test its requirement for transactivation of the HSP27 promoter. EMSA was used to demonstrate direct binding of Brn-3b to a specific site in the HSP27 promoter. Chromatin immunoprecipitation (ChIP) was performed to show that Brn-3b was associated with the HSP27 promoter in intact cells. 
Results Brn-3b protein levels correlated strongly with HSP27 levels in a significant number of breast cancer biopsies $(R=0.87)$ as well as in the breast cancer cell line, MCF7. Overexpression of Brn-3b in MCF7 cells resulted in increased HSP27 protein levels while reducing Brn-3b proteins using antisense correlated with decreased HSP27 compared with controls. Co-transfection analysis using a HSP27 reporter construct showed that Brn-3b could directly transactivate HSP27 promoter but cooperated with the ER for maximal expression. Decreasing Brn-3b using targeted RNA interference prevented activation of the HSP27 promoter by Brn-3b alone but also attenuated the response by ER. The Brn-3b site in the HSP27 promoter is flanked by two sequences that constitute half estrogen receptor elements. Site-directed mutagenesis demonstrated that this DNA sequence was required for maximal transactivation while the ChIP assay showed that Brn-3b protein binds to the HSP27 promoter in vivo.

Conclusion The ability of Brn-3b to increase expression of proteins such as HSP27 in cancer cells may help to understand the altered growth and migration of tumour cells with elevated Brn-3b proteins. Therefore factors such as Brn-3b, which increase the expression of HSP27 in breast cancers, are likely to affect the progression of diseases, prognosis and outcome of treatment.

Acknowledgements The authors thank $\mathrm{Dr}$ Daniel Ndisang, $\mathrm{Dr}$ Chandrakant Patel, Dr Jonothan Dennis and Dr Corrado D'Arrigo. This work was supported by the Breast Cancer Campaign (BCC) UK and the Association for International Cancer Research (AICR), UK.

References

1. Irshad S, Pedley RB, Anderson J, Latchman DS, BudhramMahadeo $\mathrm{V}$ : The Brn3b transcription factor regulates the growth, behaviour, and invasiveness of human neuroblastoma cells in vitro and in vivo. J Biol Chem 2004, 279:21617-21627.

2. Budhram-Mahadeo V, Parker M, Latchman DS: POU transcription factors Brn3a and Brn3b interact with the estrogen receptor and differentially regulate transcriptional activity via an estrogen response element. Mol Cell Biol 1998, 18:1029-1041.

3. Samady L, Dennis J, Budhram-Mahadeo V, Latchman DS: Activation of CDK4 gene expression in human breast cancer cells by the Brn3b POU family transcription factor. Cancer Biol Ther 2004, 3:317-323.

4. Lee S, Ndisang D, Patel C, Dennis JH, D'Arrigo C, Farooqui-Kabir $S$, Heads RJ, Latchman DS, Budhram-Mahadeo V: Expression of the Brn3b transcription factor correlates with expression of HSP27 in breast cancer biopsies and is required for maximal activation of the HSP27 promoter. Cancer Res 2005, 65:30723080.

\section{P6.01}

\section{Identification of drug targets for the treatment of Basal-like tumors KA Hoadley', CM Perou ${ }^{1,2}$}

${ }^{1}$ Curriculum in Genetics and Molecular Biology, and ${ }^{2}$ Department of Genetics and Pathology and Laboratory Medicine, University of North Carolina, Chapel Hill, North Carolina, USA

Breast Cancer Research 2005, 7(Suppl 2):P6.01 (DOI 10.1186/bcr1191) Genomic studies have identified at least five distinct subtypes of breast tumors [1]. These subtypes are believed to develop from different epithelial cell types and show different overall survival outcomes. Of particular interest is the estrogen receptor (ER)-negative Basal-like subtype, which accounts for $10-15 \%$ of all breast tumors and shows poor outcomes. In the breast cancer clinic, there are currently two biologically directed therapies that target either the ER or HER2 proteins. The Basal-like tumors lack both of these proteins [2], and hence the only treatment options for these patients are cytotoxic chemotherapies. A goal of ours was therefore to use primary breast tumor gene expression data and cell line models to identify and validate candidate biologically-based therapies for Basal-like tumors.

To identify potential targets, the gene expression data for approximately 1500 drug targets were examined across a breast tumor data set of 150 samples. Squalene epoxidase (SQLE) was expressed in most
Basal-like tumors, as well as in the Basal-like tumor-derived cell lines SUM102 and SUM149. SQLE is an attractive target because it is highly expressed, it is a rate-limiting step in the cholesterol biosynthetic pathway, and there is an available inhibitor (NB598) [3]. Recent studies using inhibitors of HMGCoA reductase (the first rate-limiting step) in epithelial cell lines suggest that inhibition of this pathway may be a potential target for therapeutic intervention [4].

Using the SUM102 and SUM149 cell lines and two more widely used luminal/ER+ lines (MCF-7 and ZR-75-1), we treated cells with NB598 and separately with lovastatin (an HMGCoA reductase inhibitor) and determined their sensitivity by identifying their 72-hour $\mathrm{IC}_{50}$ dose. Sensitivity was similar across three of the four cell lines for NB598, with the exception of SUM102, which was approximately 300 times more sensitive. Conversely, sensitivity to lovastatin was similar across three of the four cell lines except MCF-7, which was approximately five times more resistant. Since many drugs are rarely used as single agents, we also looked at the interactions between these two inhibitors and commonly used chemotherapeutics. Drug-combination sensitivities again varied across the four cell lines; however, it appears that combinations of NB598 and 5-fluorouracil were typically synergistic, while combinations with carboplatin or paclitaxel were typically antagonistic. Similar analyses are being performed for lovastatin/ chemotherapy combinations. Gene expression responses of these cell lines were also assayed using DNA microarrays. The effect on the cholesterol pathway showed that, for MCF-7 and SUM102, adding either inhibitor greatly induced most genes in the cholesterol biosynthetic pathway, while SUM149 treated with lovastatin showed induction of the pathway but treatment with NB598 did not. ZR-75-1 treated with either drug showed a slight reduction in expression of the pathway. These in vitro data suggest that inhibition of SOLE activity can reduce cell line proliferation rates and, in some instances, was synergistic with chemotherapy. These data also suggest that inhibition of the cholesterol pathway by addition of HMGCoA reductase inhibitors is different from inhibition of the pathway with SOLE inhibitors.

\section{References}

1. Sørlie T, Tibshirani R, Parker J, Hastie T, Marron JS, Nobel A Deng S, Johnsen H, Pesich R, Geisler S, et al.: Repeated observation of breast tumor subtypes in independent gene expression data sets. Proc Natl Acad Sci USA 2003, 100:8418-8423.

2. Nielsen TO, Hsu FD, Jensen K, Cheang M, Karaca G, Hu Z, Hernandez-Boussard T, Livasy C, Cowan D, Dressler L, et al.: Immunohistochemical and clinical characterization of the basal-like subtype of invasive breast carcinoma. Clin Cancer Res 2004, 10:5367-5374.

3. Chugh A, Ray A, Gupta JB: Squalene epoxidase as hypocholesterolemic drug target revisited. Prog Lipid Res 2003, 42:3750.

4. Mueck AO, Seeger H, Wallwiener D: Effect of statins combined with estradiol on the proliferation of human receptor-positive and receptor-negative breast cancer cells. Menopause 2003, 10:332-336.

\section{P6.02}

\section{Determining the factors affecting breast cancer} infectivity by oncolytic adenovirus

\section{Mysinger, C Soria, J Yeh, C O'Shea, L Timmerman,}

\section{F McCormick}

Cancer Research Institute, UCSF Comprehensive Cancer Center, San Francisco, California, USA

Breast Cancer Research 2005, 7(Suppl 2):P6.02 (DOI 10.1186/bcr1192) Cancer is the second leading cause of death in the United States. Traditional treatments for cancer, such as radiation and chemotherapy, are frequently ineffective and are often associated with painful side effects that diminish the quality of life for patients. New strategies for the treatment of cancer are greatly needed. Oncolytic viruses represent a new class of anticancer agents with the potential to greatly improve cancer treatment. 
Genetically modified adenoviruses - specifically, adenovirus serotype 5 (Ad5) - are commonly used to generate oncolytic viruses. These adenoviruses are replication-selective, meaning that they have been engineered to replicate only in cancer cells bearing certain mutations. For example, ONYX-015 is a mutant adenovirus designed to exploit the loss of functional $\mathrm{p} 53$, a loss common to many cancer cells, in order to selectively destroy malignant cells [1]. Although the use of oncolytic viruses holds great promise for cancer therapy, the success of this strategy depends on the ability of adenovirus to infect cancer cells. We are using a panel of 50 breast cancer cell lines to study Ad5 infectivity. Affymetrix array data and CGH data have been collected for all of these cell lines. We have found that the ability of Ad5 to infect these cell lines is highly variable. CAR and $\alpha v$ integrins are known to be required for Ad5 entry. However, the infectivity of the breast cancer cell lines does not correlate with CAR levels or $\alpha v$ integrin levels. For example, BT474 cells appear to have ample CAR expressed on the surface but are infected at very low rates. Alternatively, both HCC 2185 cells and MDA MB 435 cells express very little CAR on the surface but are highly infectible. We are currently investigating the possibility that other cellular factors are influencing the ability of Ad5 to infect breast cancer cells.

Reference

1. Bischoff JR, Kirn DH, Williams A, Heise C, Horn S, Muna M, Ng L, Nye JA, Sampson-Johannes A, Fattaey A, McCormick F: An adenovirus mutant that replicates selectively in p53-deficient human tumor cells. Science 1996, 274:373-376.

\section{P7.01}

\section{Magnetic resonance spectroscopy of breast cancer tissue used for tumor classification and lymph node prediction}

B Sitter ${ }^{1}$, TF Bathen 1 , LR Jensen ${ }^{1}$, D Axelson ${ }^{2}$, J Halgunset ${ }^{3}$, HE Fjosne ${ }^{4}$, S Lundgren ${ }^{5}$, IS Gribbestad 1

${ }^{1}$ Department of Circulation and Medical Imaging, NTNU, Trondheim, Norway; ${ }^{2}$ MRI Consulting, Kingston, Ontario, Canada; ${ }^{3}$ Department of Histology, ${ }^{4}$ Department of Surgery and ${ }^{5}$ Cancer Clinic, St Olavs Hospital Trondheim University Hospital, Norway

Breast Cancer Research 2005, 7(Suppl 2):P7.01 (DOI 10.1186/bcr1193)

Background The treatment plan for a breast cancer patient is based on tumor size and grade, lymph node involvement and steroid hormone receptors. Lymph node status is the strongest prognostic factor for breast cancer patients. About 25\% of node-negative patients experience recurrence or metastasis [1]. Additional methods might be important for better treatment strategies. Malignant cells have an altered metabolism, and metabolic mapping might become a tool in cancer diagnostics. High-resolution magic angle spinning (HR-MAS) magnetic resonance (MR) spectroscopy of tissue biopsies provides detailed information on their metabolic composition [2]. The aim of this study was to compare MR spectroscopic findings from breast cancer tissue with histological grading of tumor and patient lymph node status. Methods Breast cancer and non-involved adjacent tissue were excised from patients with palpable breast cancer diagnosed as invasive ductal carcinoma (IDC). Tissue specimens were analyzed in $\mathrm{D}_{2} \mathrm{O}-\mathrm{PBS}$ in a $50 \mu \mathrm{l}$ MAS rotor (4 mm o.d.). HR-MAS MR spectra were recorded on a BRUKER AVANCE DRX600 spectrometer at $4^{\circ} \mathrm{C}$. The samples were spun at $5 \mathrm{kHz}$. Proton MR spin echo spectra were acquired with a total echo time of $285 \mathrm{~ms}$ and presaturation of the water peak. A pathologist scored the relative areas of normal and neoplastic elements visually after MR analysis. Samples with less than 5\% tumor content were excluded. This resulted in a final database consisting of 115 samples: 48 non-involved tissue and 69 IDCs (grade I [ $n=4]$, grade II [ $n=33]$ and grade III $[n=32]$ ). Of the 69 tumor samples, 37 were from patients with no spread and 32 samples were from patients with lymphatic spread of cancer cells. The spectral region $2.9-4.8 \mathrm{ppm}$ was selected for principal component analysis (PCA). Two sample sets were used as PCA input: all samples $(n=115)$ and tumor samples $(n=69)$. Classification of IDC groups (grade I, grade II and grade III)

\section{Figure 1}

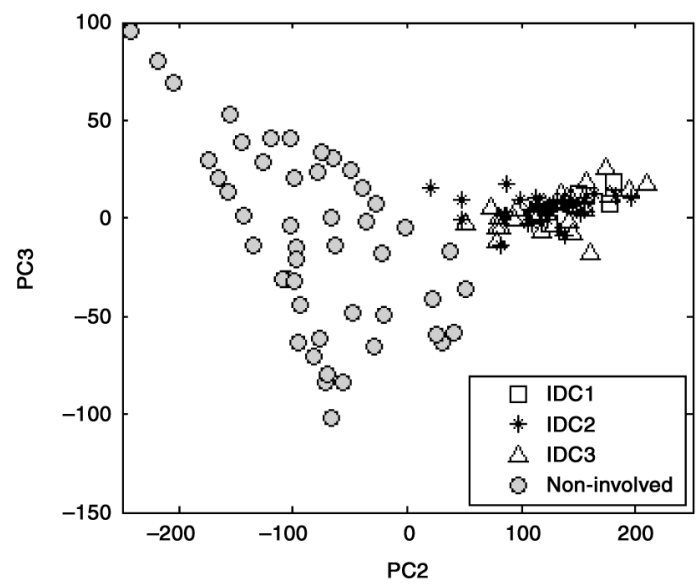

Principal component analysis score plot of all samples $(n=115)$. Labeling of samples from patient diagnosis.

\section{Figure 2}

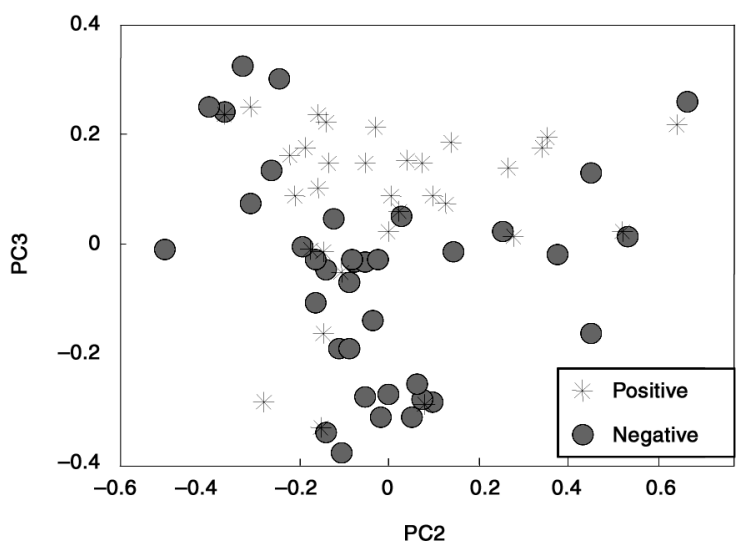

Principal component analysis score plot of tumor samples $(n=69)$. Labeling of samples from patient lymph node status.

(PNN) strategy [3]. The 25 first principal component (PC) scores from PCA of tumor samples were used as the input in PNN. Both PCA and PNN were performed with full cross-validation.

Results The 2D score plot of PC2 and PC3 from the PCA of all samples is shown in Fig. 1. All samples from non-involved tissue are clearly separated from tumor samples. Tumor samples intersperse with no possibility to differentiate among the three types of grading. The PNN of spectra from tumor samples resulted in true classification of 56 of the 69 samples with respect to grading, whereas two samples were not classified. The specificity and sensitivity of classification exceeded $80 \%$ for all groups.

A PCA score plot of PC2 and PC3 for tumor samples is shown in Fig. 2. A trend of clustering with respect to lymph node status can be seen. Classification results of node-positive and node-negative samples using PNN is presented in Table 1. Samples from patients with spread of cancer cells to lymph nodes can be predicted with a specificity of $97 \%$ and a sensitivity of $92 \%$.

Conclusion PCA led to a complete separation of the non-involved and cancerous samples. The metabolism of cancerous tissue is clearly different from non-involved tissue. Samples from lymph node-positive 
Table 1

Classification results of lymph node status from the probabilistic neural network

\begin{tabular}{lccc} 
Classification & Actual negative & Actual positive & Total \\
\hline Negative & 34 & 1 & 35 \\
Positive & 3 & 31 & 34 \\
Total & 37 & 32 & 69 \\
\hline
\end{tabular}

Sensitivity: 97\%, specificity: $92 \%$.

and lymph node-negative patients could not be separated by PCA, while PNN led to classification of the two groups with misclassification of only four samples. Metabolic patterns in breast tumors from patients with lymphatic spread differ from those without lymphatic spread. These findings show that HR-MAS of breast cancer biopsies has the potential of becoming a diagnostic tool.

\section{References}

1. Noguchi M: Therapeutic relevance of breast cancer micrometastases in sentinel lymph nodes. Br J Surg 2002, 89:1505-1515.

2. Sitter B, Sonnewald U, Spraul M, Fjosne HE, Gribbestad IS: High-resolution magic angle spinning MRS of breast cancer tissue. NMR Biomed 2002, 15:327-337.

3. Specht DF: Probabilistic neural networks. Neural Network 1990, 3:109-118.

\section{P7.02}

\section{Potentiated phospho-protein networks in cancer cells} JM Irish', R Hovland², PO Krutzik', OD Perez', O Bruserud², BT Gjertsen², GP Nolan'1

${ }^{1}$ Department of Microbiology \& Immunology, Baxter Laboratory of Genetic Pharmacology, Stanford University, Stanford, California, USA; 2Institute of Medicine, Hematology Section, University of Bergen, and Department of Internal Medicine, Hematology Section, Haukeland University Hospital, Bergen, Norway

Breast Cancer Research 2005, 7(Suppl 2):P7.02 (DOI 10.1186/bcr1194)

Altered growth factor responses in phospho-protein-driven signaling networks are crucial to cancer cell survival and pathology. Profiles of cancer cell signaling networks might therefore identify mechanisms by which such cells interpret environmental cues for continued growth. Using multiparameter flow cytometry, we monitored phospho-protein responses to environmental cues in acute myeloid leukemia at the single cell level. By exposing cancer cell signaling networks to potentiating inputs, rather than relying upon the basal levels of protein phosphorylation alone, we could discern unique cancer network profiles that correlated with genetics and disease outcome. Strikingly, individual cancers manifested multiple cell subsets with unique network profiles, reflecting heterogeneity at the level of signaling response. The results revealed a dramatic remodeling of signaling networks in cancer cells. Thus, single cell measurements of phospho-protein responses reveal shifts in signaling potential of a phospho-protein network, allowing for categorizing of cell network phenotypes by multidimensional molecular profiles of signaling.

Reference

1. Irish JM, Hovland R, Krutzik PO, Perez OD, Bruserud O, Gjertsen BT, Nolan GP: Single cell profiling of potentiated phosphoprotein networks in cancer cells. Cell 2004, 118:217-228.

\section{P7.03}

\section{A model of the BRCA1/BRCA2 network}

MA Pujana1, J-DJ Han', LM Starita'2, M Tewari', JS Ahn', V Assmann'3 ${ }^{3}$ WM EIShamy', J-F Rual', R Gelman'4, K Gunsalus ${ }^{5}$, R Greenberg ${ }^{1}$, B Bohian', N Bertin ', N Ayivi-Guedehoussou', KL Nathanson ${ }^{6}$, BL Weber ${ }^{6}$, DE Hill' ${ }^{1}$, DM Livingston', JD Parvin², M Vidal'

${ }^{1}$ Department of Cancer Biology, Dana-Farber Cancer Institute, Boston, Massachusetts, USA; ${ }^{2}$ Department of Pathology, Harvard Medical School, Brigham and Women's Hospital, Boston, Massachusetts, USA; ${ }^{3}$ Center for experimental Medicine, Institute of Tumor Biology, University Hospital Hamburg-Eppendorf, Hamburg, Germany; ${ }^{4}$ Department of Biostatistical Science, Dana-Farber Cancer Institute, Boston, Massachusetts, USA; ${ }^{5}$ Department of Biology, New York University, New York, USA; ${ }^{6}$ Department of Hematology/Oncology, University of Pennsylvania Medical Center, Philadelphia, Pennsylvania, USA

Breast Cancer Research 2005, 7(Suppl 2):P7.03 (DOI 10.1186/bcr1195) Many genes/proteins have been involved in cellular transformation. However, a systems-level understanding of this pathological process is still absent. To address this question we developed a strategy to generate preliminary models of the networks around known cancer gene products. By examining functional genomic information as gene expression profiles, disease-associated genetic networks and systemslevel integrated networks, we defined a 'breast cancer gene module' with predicted novel functional relationships to known breast cancer tumor suppressors. Genes within this module encoded for novel functional relationships with BRCA1 and BRCA2. Among the novel components identified we functionally characterized the hyaluronanmediated motility receptor (HMMR, human Rhamm), which defines a BRCA1/BRCA2 protein network involved in the control of centrosome number and chromosome segregation. Biochemical data reveal that BRCA1/BRCA2 and HMMR form complexes, that HMMR is ubiquitinated by BRCA1/BARD1, and that BRCA1 and HMMR together regulate centrosome duplication in tissue culture cell lines derived from breast tissue. Our results indicate that similar strategies could help to build and complete other cancer-related cellular networks, and thus to understand how they are affected and/or contribute to cellular transformation.

\section{P7.04}

\section{High-throughput experimental verification of predicted tissue-specific and tumor-specific splice isoforms}

\section{Zink', R Salowsky², S Haas ${ }^{3}$, C Buhlmann'2, B Korn'1}

${ }^{1}$ Agilent Technologies Deutschland GmbH, Waldbronn, Germany; ${ }^{2} R Z P D$ Ressourcenzentrum für Genomforschung, Heidelberg, Germany; ${ }^{3}$ Max-Planck-Institute for Molecular Genetics, Berlin, Germany

Breast Cancer Research 2005, 7(Suppl 2):P7.04 (DOI 10.1186/bcr1196) Alternative splicing of transcripts may lead to different mRNA species and therefore to potentially different proteins. Any failure or error in the splicing control mechanism can be involved in a number of pathological processes, such as cancer. Splice isoforms that are disease specific could therefore serve as excellent diagnostic markers, which are easily identifiable by PCR.

Computational prediction of alternative splice variants has been highly facilitating the identification of novel splice isoforms. Our prediction strategy is based on the genomic mapping (SpliceNest) of EST consensus sequences and library annotation provided in the GeneNest database. This revealed 427 genes with at least one tissue-specific transcript as well as 1120 genes showing tumor-specific isoforms. Out of these genes, a subset of predicted isoforms was experimentally verified by an RT-PCR screening approach. We have set up an experimental strategy that allows us to screen expression of genes in up to 112 different human tissues of multiple developmental stages and cell lines. Within this project, the electrophoretic separation of RT- 
PCR products turned out to be the bottleneck impeding the switch from a medium-throughput to a high-throughput strategy. To circumvent the limitations of DNA slab gel analysis, a laboratory prototype of an automated on-chip electrophoresis system that allows high-throughput analysis of DNA fragments was implemented in the workflow. In our experimental set-up, we analyzed RT-PCR samples on $4 \times 96$-well plates within a defined sequence of consecutive one-onone measurements. The high-throughput experimental verification of computationally predicted tissue specific isoforms revealed a high success rate in confirming their expression in the respective tissue. However, low expression levels of the respective transcript and the limited sensitivity of the experimental method can explain failed detection of the restricted expression pattern.

The combination of computational prediction of alternative splicing events with high-throughput experimental verification facilitates the efficient detection of tissue-specific and tumor-specific transcripts.

\section{P7.05}

RNA integrity number: towards standardization of RNA quality assessment for better reproducibility and reliability of gene expression experiments

\section{S Lightfoot, R Salowsky, C Buhlmann}

Agilent Technologies, Waldbronn, Germany

Breast Cancer Research 2005, 7(Suppl 2):P7.05 (DOl 10.1186/bcr1197)

Good RNA quality assessment is considered one of the most critical elements to obtain meaningful gene expression data via microarray or real-time PCR experiments. Advances in microfluidic technology have improved RNA quality measurements by allowing a more detailed look at patterns of RNA degradation via the use of electrophoretic traces. However, the interpretation of such electropherograms still requires a certain level of experience and can vary from one researcher to the next. The 'RNA integrity number' (RIN) algorithm is introduced to assign a user-independent integrity number to each RNA sample. The RIN has been developed using neural networks by 'teaching' this algorithm with a large number of RNA integrity data. The RIN score, based on a quality numbering system from 1 to 10 (in ascending quality), facilitates the classification of RNA samples to be used in the context of the gene expression workflow. It was found that the RIN is more reliable than the ribosomal ratio when assessing the integrity of RNA samples. The RIN is shown to be largely independent of RNA concentration, independent of instrument (Agilent 2100 bioanalyzer), and most importantly independent of the origin of the RNA sample. Using the RIN, researchers can work towards standardization of RNA integrity measurement, ensuring reproducibility and reliability of gene expression experiments. 